\title{
Tópicos em Condições de Otimalidade para Otimização não linear
}

\author{
José Alberto Ramos Flor \\ TESE APRESENTADA \\ $\mathrm{AO}$ \\ Instituto DE MATEMÁticA E EstatísticA \\ DA \\ Universidade DE SÃo PAUlo \\ PARA \\ OBTENÇÃO DO TÍTULO \\ $\mathrm{DE}$ \\ DOUTOR EM CIÊNCIAS \\ Programa: Matemática Aplicada \\ Orientador: Prof. Dr. Paulo José da Silva e Silva \\ Co-orientador: Prof. Dr. Gabriel Haeser
}

Durante o desenvolvimento deste trabalho o autor recebeu auxílio financeiro da CAPES.

São Paulo, 28 de Janeiro de 2016 


\section{Tópicos em Condições de Otimalidade para Otimização não Linear}

Esta versão da tese contém as correções e alterações sugeridas pela Comissão Julgadora durante a defesa da versão original do trabalho, realizada em 28/01/2016. Uma cópia da versão original está disponível no

Instituto de Matemática e Estatística da Universidade de São Paulo.

Comissão Julgadora:

- Prof. Dr. Paulo José da Silva e Silva (orientador)- IMECC-UNICAMP

- Prof. Dr. Carlos Humes Junior - IME-USP

- Prof. Dr. Clóvis Gonzaga - UFSC

- Prof. Dra. Sandra Augusta Santos - IMECC-UNICAMP

- Prof. Dr. Ademir Ribeiro - UFPR 
Em memoria de mi padre, José Melquiades Ramos Cegueri. 


\section{Agradecimentos}

Com esta tese, termina uma etapa da minha vida. Termina a etapa de estudante e começa de professor e de pesquisador. Acredito que toda tese de doutorado é feito com suor, trabalho duro e uma pitada de sorte. Esta tese não é diferente das outras neste quesito. Foi um longo processo, onde muitas pessoas influenciaram de uma ou outra forma, minha vida e o desenvolvimento da tese. Mencionar todas as pessoas que me apoiaram na realização desta tese seria um trabalho impossível.

Primeiro agradeço a Deus, toda vitoria pertence a ele.

Agradeço de coração, a meu amor Paloma, que sempre esteve comigo durante todo o doutorado, Obrigado pelo apoio, pela compressão, pela paciência. Também por ser o alicerce da minha família. Por cuidar de dois incentivos da minha vida, a Mel e o Pedro.

Toda mi gratitud a mi familia que siempre me apoyo en este projecto, en especial a mi madre Maria y mi padre José. Toda mi educación es fruto del amor de mis padres. Toda mi admiración a ellos. Agradezco también a mis hermanos Juan y Miguel, a mis tias, Sara y Leo que me cuidaron cuando estaba en Perú, y que siempre confiaron en mi.

Agradeço aos professores que conheci durante o doutorado, em especial ao Gabriel, Mário, Nino e ao Paulo. Agradeço a Paulo pela confiança, pelo seu exemplo como profissional. Obrigado pela ajuda e pelo incentivo para participar aos seminários de otimização. Ao Gabriel, pelos seus conselhos e pela a sua disposição de tempo para discutir minhas ideias. Ao Nino pela confiança, por acreditar fortemente na qualidade do meu trabalho, e pela sua empolgação quando transmite suas ideias. Ao Mário pelos conselhos e pelo exemplo de pesquisador. Também, agradeço ao Alfredo, pela confiança depositada em mim e toda a ajuda oferecida.

Agradeço a todos os amigos que conheci em São Paulo, a cidade da garoa. Minha gratidão aos amigos da UNI desde os tempos de estudante de graduação em Matemática.

Agradeço ao programa de pós-graduação em Matemática Aplicada do IME-USP, por ter me oferecido a oportunidade de realizar este doutorado. Agradeço também à CAPES pelo auxílio financeiro. 


\section{Resumo}

Ramos, A. Tópicos em Condições de Otimalidade para Otimização não Linear. 2015.

Tese (Doutorado) - Instituto de Matemática e Estatística, Universidade de São Paulo, São Paulo, 2015.

Esta tese é um estudo acerca da análise de convergência de vários métodos numéricos de primeira e de segunda ordem para resolver problemas de programação matemática e as condições de otimalidade associadas.

Nossas principais ferramentas são as condições sequenciais de otimalidade. As condições sequenciais de otimalidade oferecem um quadro teórico para a análise de convergência para várias famílias de métodos de primeira ordem sob condições de qualificações fracas. Nesta tese, apresentamos, para cada condição sequencial de otimalidade, a condição de qualificação mínima associada e mostramos as relações com outras condições de qualificação conhecidas. Este fato tem implicações práticas, uma vez que enfraquece as hipóteses requeridas para a convergência de vários métodos numéricos cujos critérios de paradas estão associados às condições sequenciais de otimalidade. Ainda mais, esse tipo de resultado não pode ser melhorado usando outras condições de qualificações.

Nós estendemos a noção de condições sequenciais de otimalidade de primeira ordem, para incorporar informações de segunda ordem. Apresentamos, segundo nosso conhecimento, a primeira condição sequencial de otimalidade de segunda ordem, adequada para a análise de convergência de vários métodos numéricos com convergência a pontos estacionários de segunda ordem, como por exemplo métodos baseados no lagrangeano aumentado, regiões de confiança e SQP regularizado. Associada com a nova condição sequencial de segunda ordem, temos uma nova condição de qualificação, mais fraca que as outras condições de qualificações utilizadas para a análise de convergência para métodos numéricos de segunda ordem. Nós situamos essa nova condição de qualificação com respeito a outras condições de qualificação usadas em análise de convergência. Finalmente apresentamos outra razão pela qual a condição fraca necessária de segunda ordem é a condição de segunda ordem adequada quando lidarmos com a convergência de algoritmos práticos.

Palavras-chave: otimização não-linear, condições de otimalidade, condições sequenciais, condições de qualificação, KKT, Aproximadamente KKT. 


\section{Abstract}

Ramos, A. Topics in Optimality Conditions for Nonlinear Optimization. 2015.

Thesis (Ph.D) - Instituto de Matemática e Estatística, Universidade de São Paulo, São Paulo, 2015.

This thesis deals with the convergence analysis for several first-and-second-order numerical methods used to solve mathematical programming problems.

Our main tools are the sequential optimality conditions. First-order sequential optimality conditions offer a framework to the study of the convergence analysis of several families of first-order methods, under weak constraint qualifications. In this thesis, we will introduce, for each sequential optimality condition the minimal constraint qualifications associated with it and we will show their relationships with other constraint qualifications. This fact has a practical aspect, since, we improve the convergence analysis of practical methods with stopping criteria associated with sequential optimality conditions. This results can not be improved by using another weak constraint qualifications.

We will extend the notion of first-order sequential optimality conditions to incorporate secondorder information. We will introduce, to the best of our knowledge, the first second-order sequential optimality condition, suitable to the study of the convergence analysis of several second-order methods including methods based on the augmented lagrangian, trust-region and regularized SQP. Associated with the second-order sequential optimality condition, we have a new constraint qualification weaker than all constraint qualifications used for the convergence analysis of second-order methods. We show the relationships of this new constraint qualifications with other constraint qualifications used for algorithmic purposes. We will also present a new reason why the weak secondorder necessary condition is the natural second-order condition when we are dealing with practical numerical methods.

Keywords: nonlinear optimization, optimality conditions, sequential optimality condition, constraint qualifications, KKT, Approximate-KKT. 


\section{Sumário}

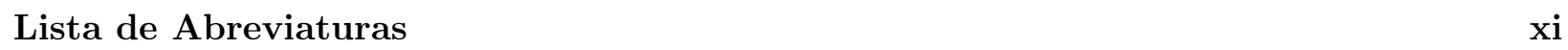

Lista de Símbolos $\quad$ xiii

Lista de Figuras $\quad$ xv

\begin{tabular}{lll}
\hline & Introdução & 1
\end{tabular}

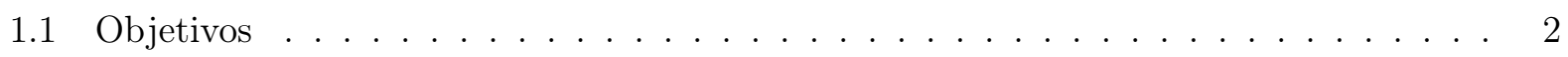

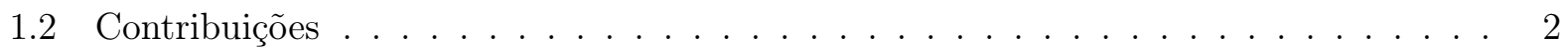

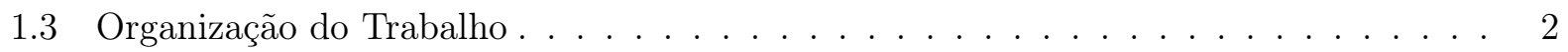

2 Condições de Otimalidade $\quad 5$

2.1 Condições de otimalidade geométricas. . . . . . . . . . . . . . . . . . . . . . . . 8

2.2 Condição de Fritz-John Generalizada . . . . . . . . . . . . . . . . . . . . . . . . . . . 12

2.3 Condições de Qualificação $\ldots \ldots \ldots \ldots$. . . . . . . . . . . . . . . . . . . 12

2.4 Condições Sequenciais de Otimalidade $\ldots \ldots \ldots \ldots$

$2.4 .1 \quad$ Condição sequencial AKKT . . . . . . . . . . . . . . . . . . . . . 17

2.4 .2 Condição sequencial AGP . . . . . . . . . . . . . . . . . . . . . . . . . . . 19

$2.4 .3 \quad$ Condição sequencial SAKKT . . . . . . . . . . . . . . . . . . . . . 22

2.4 .4 Condição sequencial L-AGP . . . . . . . . . . . . . . . . . . . . . . . . . 23

2.4 .5 Condição sequencial CAKKT . . . . . . . . . . . . . . . . . . . . . . 24

3 Condições de Qualificação associadas com as Condições Sequenciais de Otimali$\begin{array}{ll}\text { dade de primeira ordem } & 27\end{array}$

3.1 Novas condiçoes de qualificação $\ldots \ldots \ldots \ldots \ldots \ldots$. . . . . . . . . . . . . . . . . . . . .

3.1 .1 CQ associada a AKKT: Propriedade de Continuidade do Cone . . . . . . . . 28

$3.1 .2 \quad$ CQ associada a AGP $\ldots \ldots \ldots \ldots \ldots \ldots$

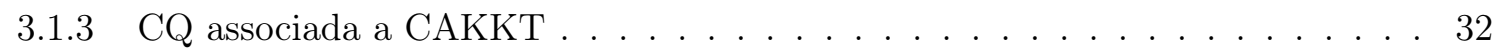

$3.1 .4 \quad$ CQ associada a SAKKT $\ldots \ldots \ldots \ldots \ldots \ldots \ldots$

3.1 .5 CQ associada a L-AGP . . . . . . . . . . . . . . . . . . . . . 35

3.2 Relações entre as novas condições de qualificação $\ldots \ldots$. . . . . . . . . . . . 36

3.3 Relações com as condições de qualificação conhecidas. . . . . . . . . . . . . . . . . . 44

3.3 .1 Relação com a condição de qualificação CPG. . . . . . . . . . . . . . . . . . 44

3.3 .2 Relação com a condição de qualificação de Abadie. . . . . . . . . . . . . . . 45

3.3 .3 Relação com a Pseudonormalidade e Quasinormalidade. . . . . . . . . . . . . . . 49 
4 Condição Sequencial de Segunda Ordem AKKT2 e condições de qualificação 53

4.1 Condição Sequencial de Segunda Ordem: AKKT2 . . . . . . . . . . . . . . . . 54

4.1 .1 Definição e Propriedades básicas . . . . . . . . . . . . . . . . . . 54

$4.1 .2 \quad$ Algoritmos práticos que geram sequências AKKT2 $\ldots \ldots \ldots$. . . . . . . 57

$4.2 \quad$ Nova CQ associada com a condição AKKT2 . . . . . . . . . . . . . . . . . 62

4.3 Relações com as CQs conhecidas . . . . . . . . . . . . . . . . . . . . 64

4.3 .1 Relação com a condição de qualificação conjunta MFCQ+WCR . . . . . . . . 64

4.3 .2 Relação com a condição de qualificação RCRCQ . . . . . . . . . . . . . . . . 64

4.3 .3 Relação com a condição de Baccari-Trad. . . . . . . . . . . . . . . . . 67

$4.4 \quad$ Nota acerca da condição WSONC $\ldots \ldots \ldots \ldots$

\begin{tabular}{|lll}
5 Conclusões & 71
\end{tabular}

$5.1 \quad$ Sugestões para Pesquisas Futuras $\ldots \ldots \ldots \ldots \ldots$. . . . . . . . . . . . . 71

\begin{tabular}{|lll}
\hline A Uma equivalência de WCR usando ferramentas de análise variacional & 73
\end{tabular}

\begin{tabular}{ll}
\hline Referências Bibliográficas & 75 \\
\hline
\end{tabular} 


\section{Lista de Abreviaturas}

AKKT Condição aproximadamente KKT (Approximate KKT)

AKKT2 Condição AKKT2 (Second-Order Approximate KKT)

AGP Condição AGP (Approximate Gradient Projection)

CAKKT Condição CAKKT (Complementary Approximate KKT)

CPG Condição do gerador de posto constante (Constant Positive Generator)

CCP Propriedade da Continuidade do Cone (Cone-Continuity Property)

CCP2 Propriedade da Continuidade do Cone de segunda ordem (Second-order Cone-Continuity Property).

CPLD Condição de Dependência Linear Positiva Constante (Constant positive linear dependence)

CRCQ Condição de qualificação do Posto Constante (Constant rank constraint qualification)

CRSC Condição de posto constante da componente subespaço (Constant Rank of the Subspace Component)

L-AGP Condição L-AGP (Linear Approximate Gradient Projection)

LICQ Condição de Independência Linear (Linear Independence)

MFCQ Condição de qualificação de Mangasarian-Fromovitz

SAKKT Condição SAKKT (Strong Approximate KKT)

SCCP2 Propriedade forte do Cone Continuo de segunda ordem (Strong Second-order Cone-Continuity Property).

SSONC Condição forte necessária de segunda ordem (Strong Second-Order Necessary Condition).

SSOSC Condição forte suficiente de segunda ordem (Strong Second-Order Sufficient Condition).

WCR Condição de posto constante fraca (weak constant rank).

WSONC Condição fraca necessária de segunda ordem (Weak Second-Order Necessary Condition). 


\section{Lista de Símbolos}

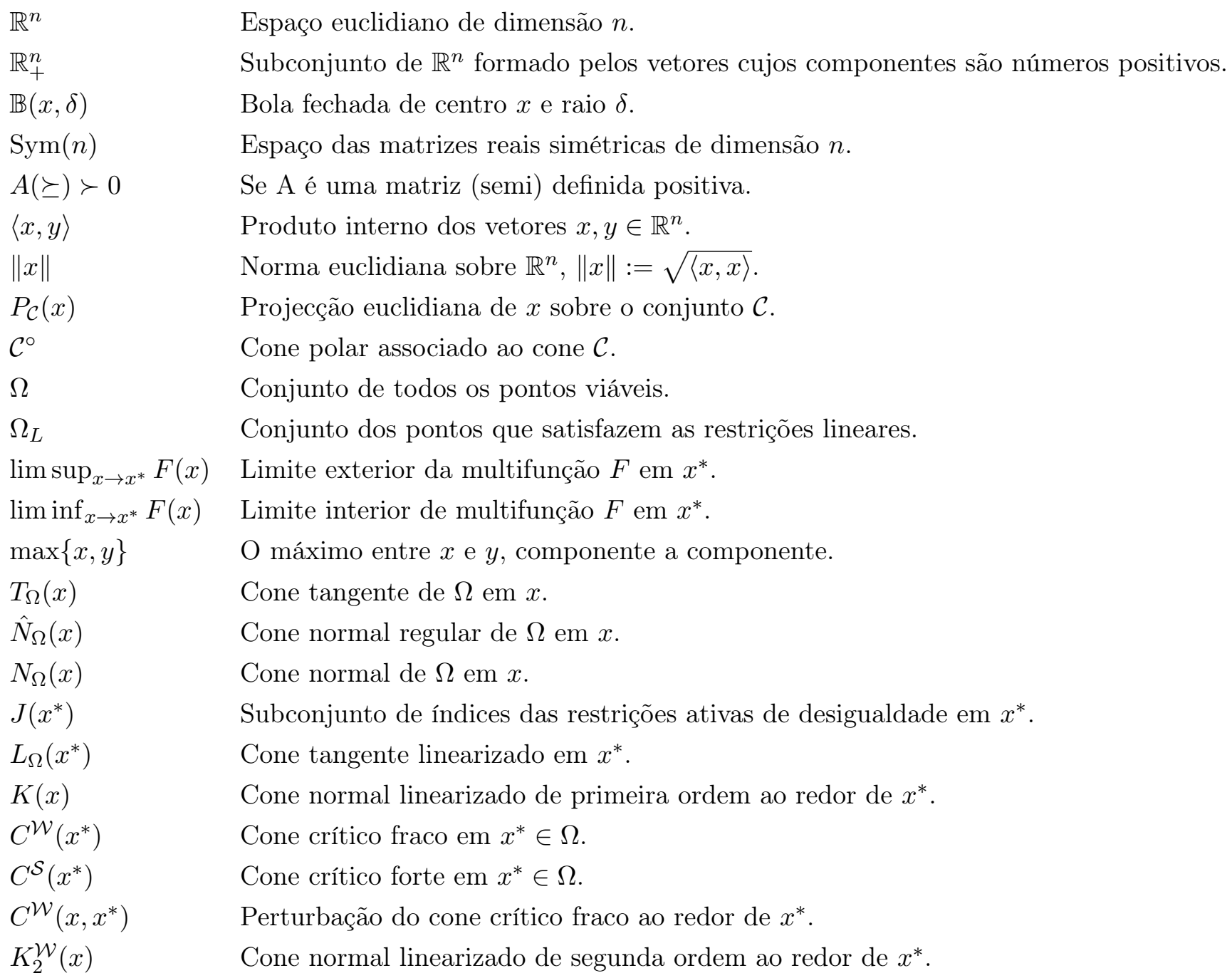




\section{Lista de Figuras}

2.1 Condição Geométrica de Otimalidade de primeira ordem. . . . . . . . . . . . . . . 10

2.2 A condição de Fritz-John não é suficiente para a otimalidade, mesmo no caso linear. 13

2.3 Decomposição de um cone em um subespaço e em um cone pontudo. . . . . . . . . . 15

2.4 Relações entre as condições de qualificação mencionadas. Como mostraremos na tese, as condições de qualificação de Pseudonormalidade, Quasinormalidade e de Abadie não são suficientes para garantir a convergência de certos métodos numéricos. . . . . . 17

2.5 Relações entre as condições sequenciais de otimalidade de primeira ordem. . . . . . . 25

$3.1 \quad$ Exemplo do cone associado à condição AKKT . . . . . . . . . . . . . . . . . . . . . . . . . . . . . . . . .

3.2 Exemplo do cone associado à condição AGP. . . . . . . . . . . . . . . . . . . . . . 32

3.3 Exemplo de cones associados com a condição CAKKT. . . . . . . . . . . . . . . . . . . . . . . . . . . . .

3.4 Exemplo do cone associado à condição SAKKT . . . . . . . . . . . . . . . . . . 35

3.5 Equivalência das novas condições de qualificação com as condições sequenciais. .. 36

3.6 Relações entre as novas condições de qualificação $\ldots \ldots \ldots$. . . . . . . . . . . . . . . . . 44

3.7 Relações das novas condições de qualficação (CCP, AGP regular, SAKKT regular, CAKKT regular e L-AGP regular) com as CQs conhecidas na literatura. . . . . . . . 51

4.1 Relações das CQs associadas com a convergência a pontos estacionários de segunda

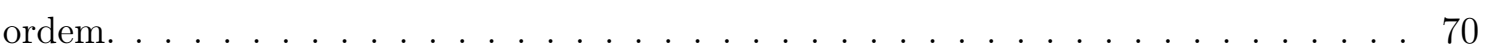




\section{Capítulo 1}

\section{Introdução}

A maioria dos métodos numéricos são de natureza iterativa. Portanto, na sua implementação, devemos decidir, em cada iteração, se é sensato parar com a execução. Usualmente essa decisão está baseada na verificação aproximada de alguma condição de otimalidade. Para métodos numéricos de primeira ordem, essa prática encontra-se teoricamente apoiada pelas condições sequenciais de otimalidade, ferramenta teórica útil no estudo de algoritmos práticos e na sua análise de convergência sob condições de qualificação mais fracas que as usuais. Podemos argumentar que a confiabilidade de um método numérico de otimização está baseada na força da condição de otimalidade associada ao critério de parada do método. Quão mais restritivo o critério de parada, melhores evidências sobre a qualidade do possível candidato a solução temos. Uma forma de analisar a força das condições de otimalidade é ver quais tipos de proposições elas implicam. Como veremos ao longo da tese, a força das condições sequenciais de otimalidade está relacionada com certas condições de qualificação e que condições de qualificação fracas implicam condições sequenciais fortes. Assim, duas perguntas surgem naturalmente: Quais são as condições de qualificação mais fracas possíveis associadas às condições sequenciais permitindo assegurar a convergência de vários métodos numéricos? É possível estender essa análise para métodos numéricos com convergência a pontos estacionários de segunda ordem? Esta tese responde afirmativamente cada uma dessas questões.

Nesta tese apresentamos uma análise completa das condições sequenciais de otimalidade e suas relações com condições de qualificação com claras implicações algorítmicas. A tese divide-se em duas partes: A primeira parte é dedicada aos métodos numéricos de primeira ordem e a segunda refere-se a métodos numéricos de segunda ordem. Para cada uma dessas partes, apresentamos os resultados de nossa pesquisa e nossas contribuições. Essas contribuições possuem implicações algorítmicas, como veremos ao longo do texto.

A primeira parte do tese refere-se a métodos numéricos de primeira ordem. O estudo teórico de métodos numéricos de primeira ordem está relacionada com as condições sequenciais de otimalidade (de primeira ordem). É conhecido que cada condição sequencial de otimalidade é uma condição de otimalidade não trivial e usada amplamente na análise de convergência de método numéricos de primeira ordem. Na primeira parte desta tese, damos uma resposta definitiva acerca de quais são as condições de qualificação mais fraca possíveis que servem para esse propósito. De fato, fornecemos, para cada condições sequencial de otimalidade, a sua medida de força mais precisa, através de uma condição de qualificação. Cada uma dessa condições de qualificação cumpre com respeito à condição sequencial associada o mesmo papel que a condição de Guignard cumpre com respeito à otimalidade. Isto tem consequência algorítmicas, porque oferece o grau de confiabilidade associado a cada critério de parada de vários métodos numéricos e garante convergência a pontos estacionários de primeira ordem sob novas condições de qualificação. Esses resultados não podem ser melhorado mediante o uso de outras condições de qualificação.

Na segunda parte da tese, apresentamos, pela primeira vez uma condição sequencial de otimalidade de segunda ordem, que denominamos de aproximadamente KKT de segunda ordem (AKKT2), abrindo a porta para uma unificação da análise de convergência de vários métodos numéricos com convergência a pontos estacionários de segunda ordem. Acreditamos que a condição AKKT2 pode 
servir para o desenho de algoritmos com convergência a pontos estacionários de segunda ordem. Junto com a condição AKKT2, apresentamos a condição de qualificação associada, CCP2. Situamos especificamente o lugar da CCP2 com respeito às outras condições de qualificação de segunda ordem conhecidas na literatura e usadas na analise de convergência. Essa abordagem, nos permite mostrar que a condição RCRCQ pode ser usada na análise de convergência, não só de um método, mas de uma família de métodos (a família formada pelos métodos que geram sequências AKKT2), e como consequência podemos garantir a convergência a pontos estacionários de segunda ordem mesmo que o conjunto de multiplicadores de Lagrange associados não seja limitado. Um fato novo na literatura, já que as hipóteses usuais pedem que os multiplicadores de Lagrange sejam limitados. Outra consequência, que nasce dessa abordagem, é fornecer um motivo pelo qual a condição fraca necessária de segunda ordem é a condição necessária de segunda ordem que funciona melhor quando analisamos métodos numéricos com convergência a pontos estacionários de segunda ordem.

\subsection{Objetivos}

O objetivo da tese é o estudo das condições sequenciais de otimalidade e de seu papel unificador no estudo da convergência de vários métodos numéricos de otimização, tanto para métodos numéricos de primeira ordem como também para métodos numéricos de segunda ordem. Este estudo é relevante, pois provamos a convergência de vários métodos numéricos usando as condições de qualificação mais fracas possíveis.

\subsection{Contribuições}

As principais contribuições da tese são as seguintes:

1. Fornecemos uma nova condição de qualificação para cada condição sequencial de otimalidade. Essa condição de qualificação, fornece a medida mais precisa da força da condição sequencial de otimalidade e, como consequência, do critério de parada associado. Cada uma dessas condições de qualificação cumpre o mesmo papel, com respeito à respectiva condição sequencial que a condição de qualificação de Guignard cumpre com respeito à otimalidade.

2. Apresentamos a primeira condição sequencial de segunda ordem. Mostramos, que ela é uma verdadeira condição de otimalidade e está relacionada com os critérios de paradas de vários métodos numéricos os quais incluem os métodos de lagrangiano aumentado, os métodos de programação quadrática sequencial regularizados e os métodos de regiões de confiança.

3. Associada à nova condição sequencial de otimalidade de segunda ordem, apresentamos uma nova condição de qualificação. Essa condição de qualificação permite unificar a análise de convergência de vários algoritmos práticos e é a condição de qualificação mais fraca possível. Além disso, localizamos essa nova condição de qualificação com respeito às outras condições de qualificação.

\subsection{Organização do Trabalho}

No capítulo 2, fazemos uma revisão das condições de otimalidade, das principais condições de qualificação encontradas na literatura e, finalmente, das condições sequenciais de otimalidade de primeira ordem, como por exemplo, AKKT, AGP, CAKKT e variantes. Nossa primeira contribuição encontra-se no capítulo 3. Neste capítulo, definimos para cada condição sequencial de otimalidade, uma nova condição de qualificação. Mostramos as relações exatas entre essas condições de qualificação e as relações com as condições de qualificação conhecidas na literatura. Por exemplo, mostramos que são mais fortes que a condição de Abadie, mais fraca que a condição CPG e independentes da pseudonormalidade e a quasinormalidade. No capítulo 4 introduzimos, segundo nosso conhecimento, 
a primeira condição sequencial adequada para o estudo de métodos numéricos com convergência a pontos estacionários de segunda ordem. Mostramos que essa condição é de verdade uma condição de otimalidade e que é gerada por vários algoritmos numéricos. Juntamente com a nossa nova condição sequencial de segunda ordem, introduzimos uma nova condição de qualificação. Mostramos que essa condição de qualificação tem claras implicações algorítmicas e situamos o seu lugar com respeito a outras condições de qualificação conhecidas. Em particular, provamos que é mais fraca que CRCQ e que MFCQ+WCR. Finalmente, no capítulo 5 discutimos algumas das conclusões obtidas neste trabalho. No apêndice A, apresentamos uma caracterização, usando a linguagem de análise variacional, da propriedade de posto constante fraca, WCR. 


\section{Capítulo 2}

\section{Condições de Otimalidade}

O problema geral de otimização consiste em

$$
\text { minimizar } f(x) \text { sujeito a } x \in \Omega \text {. }
$$

Nesta tese, $\Omega$ é um subconjunto de $\mathbb{R}^{n}$. Devemos enfatizar que o que realmente se deseja é encontrar um ponto $x^{*} \in \Omega$ tal que $f\left(x^{*}\right) \leq f(x)$ para todo $x \in \Omega$. Em outras palavras, o que realmente desejamos são minimizadores globais do problema.

Na prática, achar soluções globais é usualmente muito difícil, mesmo verificar, se um ponto $x \in \Omega$ é um minimizador global resulta, em muitos casos, impossível. A maioria dos algoritmos populares de otimização não garantem a descoberta de minimizadores globais, mas sim, pontos que têm uma boa chance de serem minimizadores globais. Assim, estamos interessados em algoritmos que podem ou não convergir a minimizadores globais. Apesar que isso pode ser considerado como uma fraqueza, o que pode ser provado sobre a convergência destes algoritmos reflete bastante bem o que acontece na prática.

Devido a que a propriedade de ser minimizador é muito ambiciosa, estamos interessados em analisar propriedades mais fracas que, no entanto, sejam satisfeitas por todos os minimizadores globais do problema. Tais propriedades se chamam de Condições Necessárias de Otimalidade (ou simplesmente Condições de Otimalidade). Os pontos que satisfazem essas propriedades se dizem estacionários. Isto é, uma condição de otimalidade caracteriza-se pela implicação

\section{Minimizador global $\Longrightarrow$ Ponto estacionário}

Condições de otimalidade tão fracas como pertencer a $\Omega$ ou mesmo a $\mathbb{R}^{n}$ não merecem maior atenção. Uma boa característica de uma condição de otimalidade é a de ser razoavelmente forte. Naturalmente, a condição de otimalidade mais forte possível é a de ser minimizador global. Mas como já foi mencionado anteriormente conseguir ou verificar se um ponto é um minimizador global é extremamente difícil. Outra condição de otimalidade é a de ser um minimizador local: um ponto $x^{*} \in \Omega$ é um minimizador local, se existe uma vizinhança de $x^{*}$ tal que $f\left(x^{*}\right) \leq f(x)$ para todo $x \in \Omega$ que pertence a tal vizinhança. Ser minimizador local é estritamente mais fraco que ser um minimizador global, porém, ele tem o empecilho de não ser, em geral, fácil de verificar, o que não é satisfatório de um ponto de vista prático.

Ao final, o que queremos são condições de otimalidades fortes cuja verificação limita-se a um cálculo. Tal requerimento é razoável. O exemplo clássico é da minimização sem restrições $\left(\Omega=\mathbb{R}^{n}\right)$. Neste caso, se a função objetivo $f$ é diferenciável e se $x^{*}$ é um minimizador local, então que $\nabla f\left(x^{*}\right)=$ 0 . Se $f$ é duas vezes diferenciável, temos que além de $\nabla f\left(x^{*}\right)=0$, a Hessiana de $f$ deve ser semidefinida positiva em $x^{*}$. Como vemos, a verificação destas condições de otimalidade só envolvem o cálculo das derivadas de $f$. Note que dependendo do uso das derivadas em sua formulação, ditas condições de otimalidade podem ser de primeira ou segunda ordem. Ordens maiores são raramente usadas na prática.

O papel das condições de otimalidade não é fazer uma lista de possíveis candidatos a solução 
e verificar cada um dos candidatos até encontrar uma solução, mas sim, justificar os algoritmos numéricos, em particular os critérios de parada, os tipos de convergência e a robusteza.

Para continuar com nossa análise, consideremos que o conjunto $\Omega$ é definido através de restrições de igualdade e desigualdade, isto é,

$$
\Omega:=\left\{x \in \mathbb{R}^{n}: h(x)=0 \text { e } g(x) \leq 0\right\},
$$

onde $g: \mathbb{R}^{n} \rightarrow \mathbb{R}^{p}$ e $h: \mathbb{R}^{n} \rightarrow \mathbb{R}^{m}$ são funções diferenciáveis com derivada contínua em $\mathbb{R}^{n}$. Escreveremos $h=\left(h_{1}, \ldots, h_{m}\right)$ e $g=\left(g_{1}, \ldots, g_{p}\right)$.

Este tipo de problema é relevante já muitos problemas científicos e tecnológicos exigem a solução de problemas desse tipo. Ver (BSS06, Bie10, NW06, Rus06).

Sabemos que para o caso de minimização irrestrita, a condição de otimalidade: "gradiente igual a zero" satisfaz os requisitos anteriores (isto é, ele é uma condição de otimalidade e sua verificação se reduz a um mero cálculo). A pergunta natural é: Qual é a condição equivalente para o caso de minimização com restrições?

$$
\begin{array}{ll}
\text { Minimizar } & f(x) \\
\text { sujeito a } & g(x) \leq 0 \\
& h(x)=0 .
\end{array}
$$

Para isto usaremos uma interpretação mecânica do problema (2.0.3). O seguinte argumento é baseado no argumento de Bonnans e Shapiro (BS98). Suponha que a função objetivo é a energia potencial de uma partícula. A força associada é dada por $-\nabla f(x)$. Cada restrição de desigualdade é considerada como uma barreira que impede a penetração num corpo rígido $g_{j}(x) \geq 0$ e cada restrição de igualdade é interpretada como um trilho onde a partícula deve estar. Associada a essa restrição $g_{j}(x)$ temos uma força de repulsão normal à superfície do corpo. Se $\nabla g_{j}(x)$ não é zero, então o vetor normal ao corpo é $-\nabla g_{j}(x)$. Para as restrições de igualdade a força de repulsão é qualquer vetor normal paralelo a $\nabla h_{i}(x)$. Tendo em consideração o fato que a força de repulsão do corpo rígido $g_{j}(x) \geq 0$ deve ser nula se a partícula não está em contato com ele, podemos escrever o equilíbrio do sistema como:

$$
\begin{gathered}
\nabla f(x)+\sum_{i=1}^{m} \lambda_{i} \nabla h_{i}(x)+\sum_{j=1}^{p} \mu_{j} \nabla g_{j}(x)=0, \\
h(x)=0, g(x) \leq 0
\end{gathered}
$$

e

$$
\mu_{j} g_{j}(x)=0, \quad \mu_{j} \geq 0 \text { para todo } j \in\{1, \ldots, p\} .
$$

Note que esta forma de derivar o sistema KKT (2.0.4, 2.0.5) e 2.0.6) ) é diferente ao usado normalmente, onde é analisado as direções normais do conjunto viável (expressão (2.1.9) ) em lugar de conjunto complementar (ambos pontos de vistas são equivalentes sob qualquer condição de qualificação).

Dizemos que um ponto $x \in \mathbb{R}^{n}$ satisfaz as condições KKT (Karush-Kuhn-Tucker) para o problema 2.0.3 se existem $\lambda \in \mathbb{R}^{m}$ e $\mu \in \mathbb{R}^{p}$ tais que as condições 2.0.4), 2.0.5) e 2.0.6) são satisfeitas. A condição (2.0.5) é a condição de viabilidade e a condição (2.0.6) chama-se de condição de complementaridade.

Lamentavelmente, não é verdade que as condições KKT são uma condição de otimalidade. De fato, temos o seguinte exemplo em $\mathbb{R}$, minimizar $x$ sujeito a $x^{2}=0$. Claramente a única solução é $x^{*}=0$ mas a condição (2.0.4) nunca é satisfeita. Assim necessitamos hipóteses adicionais para assegurar que as condições KKT sejam consideradas uma condição de otimalidade.

Uma propriedade acerca dos pontos viáveis da forma 2.0.2) e que depende somente da descrição das restrições é uma Condição de Qualificação (CQ) se quando é satisfeita em um minimizador local $x^{*}$ implica que as condições KKT valem em $x^{*}$. Assim, se CQ é uma condição de qualificação, a 
proposição

"KKT ou não CQ"

é uma condição de otimalidade. Segundo a proposição (2.0.7), condições de otimalidade fortes correspondem a condições de qualificação fracas. De fato, sejam $\mathcal{P}$ e $\mathcal{Q}$ duas condições de qualificação tal que $\mathcal{P}$ implica $\mathcal{Q},(\mathcal{P} \Rightarrow \mathcal{Q})$. Neste caso, $\mathcal{Q}$ é uma condição de qualificação mais fraca que $\mathcal{P}$. Mas, a condição de otimalidade "KKT ou não $\mathcal{Q}$ " associada a $\mathcal{Q}$ é mais forte que a condição de otimalidade "KKT ou não $\mathcal{P}$ " associada a $\mathcal{P}$. Assim, condições de qualificação fracas são preferíveis que condições de qualificação fortes pois elas implicam condições de otimalidade fortes.

A reputação das condições KKT não só se deves ao fato que elas são uma condição necessária de otimalidade sob qualquer condição de qualificação, mas também ao fato que elas são condições suficientes de otimalidade se o problema (2.0.3) é convexo, isto é, se a função objetivo bem como as funções que definem as restrições são convexas. Por exemplo, o caso de programação linear. Assim, no caso convexo, achar pontos que satisfazem as condições KKT equivale a achar minimizadores globais.

Quando as derivadas parciais de segunda ordem encontram-se disponíveis, no caso de minimização irrestrita, sabemos que podemos obter uma condição de otimalidade mais forte que o gradiente da função objetivo igual a zero: Além disso, a Hessiana da função objetivo é semi-definida positiva no ponto de análise. O que podemos dizer para o caso de minimização com restrições?

A resposta não é tão imediata como no caso das condições KKT. Várias condições de otimalidade de segunda ordem têm sido propostas na literatura, revisaremos as mais importantes.

Denotemos por $J(x):=\left\{j \in\{1, \ldots, p\}: g_{j}(x)=0\right\}$ o conjunto de índices das restrições de desigualdade ativa em $x$. Seja $L(x, \lambda, \mu)$ a função Lagrangiana associada ao problema (2.0.3):

$$
L(x, \lambda, \mu)=f(x)+\sum_{i=1}^{m} \lambda_{i} h_{i}(x)+\sum_{j=1}^{p} \mu_{j} g_{j}(x)
$$

em que $\mu_{j} \geq 0$ para todo $j \in\{1, \ldots, p\}$. Sob certas condições de qualificação podemos provar que todo minimizador local $x^{*}$ de 2.0 .3 satisfaz a condição fraca necessária de segunda ordem, Weak Second-Order Necessary optimality Condition (WSONC) pelas suas siglas em inglês, definida a seguir.

Definição 2.0.1. Um ponto viável $x^{*} \in \Omega$ satisfaz a condição fraca necessária de segunda ordem, Weak Second-Order Necessary Optimality Condition (WSONC), se existem multiplicadores de Lagrange $\lambda^{*} \in \mathbb{R}^{m}, \mu^{*} \in \mathbb{R}_{+}^{p}, \mu_{j}^{*}=0$ para $j \notin J\left(x^{*}\right)$, tais que

$$
\nabla f\left(x^{*}\right)+\sum_{i=1}^{m} \lambda_{i}^{*} \nabla h_{i}\left(x^{*}\right)+\sum_{j \in J\left(x^{*}\right)} \mu_{j}^{*} \nabla g_{j}\left(x^{*}\right)=0
$$

$e$

$$
\left(\nabla^{2} f\left(x^{*}\right)+\sum_{i=1}^{m} \lambda_{i}^{*} \nabla^{2} h_{i}\left(x^{*}\right)+\sum_{j \in J\left(x^{*}\right)} \mu_{j}^{*} \nabla^{2} g_{j}\left(x^{*}\right)\right)(d, d) \geq 0 \text { para todo } d \in \mathcal{C}^{W}\left(x^{*}\right),
$$

onde $\mathcal{C}^{W}\left(x^{*}\right)$ é o cone crítico fraco definido como

$$
\mathcal{C}^{W}\left(x^{*}\right):=\left\{d \in \mathbb{R}^{n}:\left\langle\nabla h_{i}\left(x^{*}\right), d\right\rangle=0, i \in\{1, \ldots, m\} \text { e }\left\langle\nabla g_{j}\left(x^{*}\right), d\right\rangle=0, j \in J\left(x^{*}\right)\right\} .
$$

Em outras palavras, a condição WSONC é satisfeita quando as condições KKT valem e para esses multiplicadores de Lagrange, a Hessiana da função Lagrangiana $L\left(\cdot, \lambda^{*}, \mu^{*}\right)$ é semi-definida positiva em $x^{*}$ restrita ao cone crítico fraco $\mathcal{C}^{W}\left(x^{*}\right)$. Note que o cone crítico fraco é de fato um 
subespaço linear de $\mathbb{R}^{n}$. Quando o cone crítico fraco é substituído pelo cone crítico forte

$$
\mathcal{C}^{S}\left(x^{*}, \mu^{*}\right):=\left\{\begin{array}{ll}
d \in \in \mathbb{R}^{n}: \quad & \left\langle\nabla h_{i}\left(x^{*}\right), d\right\rangle=0, \text { para } i \in\{1, \ldots, m\},\left\langle\nabla g_{j}\left(x^{*}\right), d\right\rangle=0, \text { se } \mu_{j}^{*}>0 \\
& \left\langle\nabla g_{j}\left(x^{*}\right), d\right\rangle \leq 0, \text { se } \mu_{j}^{*}=0, \quad j \in J\left(x^{*}\right)
\end{array}\right\}
$$

dizemos que a condição forte necessária de segunda ordem, Strong Second-Order Necessary optimality Condition (SSONC), vale em $x^{*}$. A condição SSONC é bem conhecida na literatura (BSS06, Ber99, FM68, Fle81). Ela é mais forte que a condição WSONC e é implicada por várias condições de qualificação quando $x^{*}$ é um minimizador local.

O cone $\mathcal{C}^{S}\left(x^{*}, \mu^{*}\right)$ está bem definido para todo $\mu^{*} \in \mathbb{R}_{+}^{p}$ e quando $\mu^{*}$ é um multiplicador de Lagrange, o cone crítico forte é equivalente ao cone

$$
\left\{d \in \mathbb{R}^{n}:\left\langle\nabla h_{i}\left(x^{*}\right), d\right\rangle=0, i \in\{1, \ldots, m\},\left\langle\nabla g_{j}\left(x^{*}\right), d\right\rangle \leq 0, j \in J\left(x^{*}\right),\left\langle\nabla f\left(x^{*}\right), d\right\rangle \leq 0\right\} .
$$

Assim, neste caso, o multiplicador é redundante e escrevemos $\mathcal{C}^{S}\left(x^{*}\right)$ em lugar de $\mathcal{C}^{S}\left(x^{*}, \mu^{*}\right)$. Sob a condição de complementaridade estrita (i.e. $\mu^{*}$ satisfaz $2.0 .9 p$ e $\mu_{j}^{*}>0$ para $j \in J\left(x^{*}\right)$ ), ambos cones $\mathcal{C}^{S}\left(x^{*}\right)$ e $\mathcal{C}^{\mathcal{W}}\left(x^{*}\right)$ coincidem e SSONC é equivalente a WSONC.

Do ponto de vista prático, verificar se a condição SSONC é satisfeita, é em geral um problema NP-difícil (MK87) e segundo nosso conhecimento, não existem algoritmos que gerem sequencias cujo pontos limites satisfazem a condição forte SSONC. Uma variação da condição SSONC é a condição forte suficiente de segunda ordem SSOSC que afirma que a Hessiana $\nabla_{x}^{2} L\left(x^{*}, \lambda^{*}, \mu^{*}\right)$ é definida positiva no cone $\mathcal{C}^{S}\left(x^{*}, \mu^{*}\right)$. A condição SSOSC é amplamente usada na análise de estabilidade e sensibilidade (FM68, Ber82, Fia83) e está relacionada com a noção de Error Bound para o sistema KKT (HG99).

A maioria das condições de otimalidade de segunda ordem usadas em algoritmos são da forma:

"Se $x^{*} \in \Omega$ satisfaz alguma condição de qualificação CQ2 então a condição WSONC vale em $x^{*}$. Assim, proposições da forma

$$
\text { "WSONC ou não CQ2" }
$$

são condições de otimalidade de segunda ordem associadas à algoritmos. Claramente condições de otimalidade fortes da forma (2.0.14 correspondem a condições de qualificação fracas. Nem todas as CQs são suficientes para afirmar a validade da condição WSONC. Por exemplo, Anistescu em (Ani00) mostrou que a condição de qualificação de Mangasarian-Fromovitz (MFCQ) não pode usada como CQ2 na expressão 2.0.14.

Nossa preferência por proposições da forma (2.0.14), vêm de considerações práticas. Por exemplo, poderíamos querer proposições da forma: "SSONC ou não CQ2". Obviamente esse tipo de proposições são mais fortes que 2.0.14), porém, elas possuem duas característica indesejáveis: Primeiro, como já mencionamos anteriormente, verificar se SSONC é satisfeita, é em geral um problema NP-difícil enquanto verificar se a condição WSONC vale, se resume a comprovar se a Hessiana em um ponto dado é semi-definida positiva sobre um subespaço. Segundo, não existem algoritmos, de acordo com nosso conhecimento, com convergência a pontos onde a condição SSONC vale, mesmo no caso de problemas bem comportados. Por exemplo, Gould e Toint (GT98) apresentaram um problema de otimização simples com caixas onde o método de barreira gera uma sequência de minimizadores que satisfazem a condição suficiente de segunda ordem SSOSC mas a condição SSONC falha no ponto limite. Nesta tese, no capítulo 4, seção 4.4 forneceremos outro motivo de porque a condição WSONC é a adequada quando tratamos de algoritmos práticos.

\subsection{Condições de otimalidade geométricas.}

Começaremos revisando os objetos geométricos mais importantes em otimização e na análise variacional (RW09, Mor06). Dizemos que $F: \mathbb{R}^{s} \rightrightarrows \mathbb{R}^{d}$ é uma multifunção se para cada $x \in \mathbb{R}^{s}$, $F(x)$ é um subconjunto de $\mathbb{R}^{d}$. A ideia de multifunção aparece naturalmente em diferentes áreas 
da Matemática, como em otimização, análise convexa, teoria de controle, análise variacional, etc. Exemplos clássicos de multifunções são os cones normais, os cones tangentes, conjunto de soluções de problemas de otimização, etc. A noção de multifunção estende naturalmente a noção usual de função.

Dada uma multifunção $F: \mathbb{R}^{s} \rightrightarrows \mathbb{R}^{d}$, o limite exterior sequencial de Painlevé-Kuratowski de $F(z)$ quando $z \rightarrow z^{*}$ é denotado por

$$
\limsup _{z \rightarrow z^{*}} F(z):=\left\{w^{*} \in \mathbb{R}^{d}: \exists\left(z^{k}, w^{k}\right) \rightarrow\left(z^{*}, w^{*}\right) \text { tal que } w^{k} \in F\left(z^{k}\right)\right\}
$$

e o limite interior por

$$
\liminf _{z \rightarrow z^{*}} F(z):=\left\{w^{*} \in \mathbb{R}^{d}: \forall z^{k} \rightarrow z^{*} \exists w^{k} \rightarrow w^{*} \text { tal que } w^{k} \in F\left(z^{k}\right)\right\} .
$$

Dizemos que $F$ é semicontínua exteriormente (osc) em $z^{*}$ se

$$
\limsup _{z \rightarrow z^{*}} F(z) \subset F\left(z^{*}\right)
$$

e $F$ é semicontinua interiormente (isc) em $z^{*}$ se

$$
F\left(z^{*}\right) \subset \liminf _{z \rightarrow z^{*}} F(z) .
$$

Quando $F$ é simultaneamente semicontínua exteriormente e semicontínua interiormente em $z^{*}$ dizemos que $F$ é contínua em $z^{*}$.

Dado um conjunto $X$ em $\mathbb{R}^{n}$ dizemos que $X$ é um conjunto localmente fechado em $x$ se existe uma vizinhança fechada $V$ de $x$ tal que $X \cap V$ é um subconjunto fechado. Claramente, $X$ é um conjunto fechado se $X$ é localmente fechado para todo $x \in \mathbb{R}^{n}$. Dado um cone $\mathcal{C} \subset \mathbb{R}^{s}$, o cone polar é definido como $\mathcal{C}^{\circ}:=\left\{v \in \mathbb{R}^{s} \mid\langle v, k\rangle \leq 0\right.$ para todo $\left.k \in \mathcal{C}\right\}$. Quando $\mathcal{C}$ é um cone convexo fechado temos $\mathcal{C}=\mathcal{C}^{\circ \circ}$. Usamos a notação $\phi(t) \leq o(t)$ para qualquer função $\phi: \mathbb{R}_{+} \rightarrow \mathbb{R}^{s}$ tal que $\lim \sup _{t \rightarrow 0_{+}} t^{-1} \phi(t) \leq 0$. Aqui, o limite superior refere-se à noção clássica de limite superior para funções reais com valores reais, isto é, o limite superior é o maior ponto de limite possível. e não ao limite superior definido para multifunções.

Seja $\Omega \subset \mathbb{R}^{n}$ e $z^{*} \in \Omega$ um ponto fixo arbitrário. O cone tangente de $\Omega$ em $z^{*}$ é definido como

$$
T_{\Omega}\left(z^{*}\right):=\limsup _{t \downarrow 0} \frac{\Omega-z^{*}}{t}=\left\{d \in \mathbb{R}^{n}: \exists t_{k} \downarrow 0, d^{k} \rightarrow d \text { tal que } z^{*}+t_{k} d^{k} \in \Omega\right\} .
$$

O cone normal regular de $\Omega$ em $z^{*} \in \Omega$ é definido por

$$
\widehat{N}_{\Omega}\left(z^{*}\right):=\left\{w \in \mathbb{R}^{n}:\left\langle w, z-z^{*}\right\rangle \leq o\left(\left|z-z^{*}\right|\right) \text { para } z \in \Omega\right\} .
$$

O cone normal limite de $\Omega$ em $x^{*} \in \Omega$ é dado por

$$
N_{\Omega}\left(z^{*}\right):=\limsup _{z \rightarrow z^{*}, z \in \Omega} \widehat{N}_{\Omega}(z)
$$

Quando $\Omega$ é um conjunto convexo, o cone regular normal (2.1.6) e cone normal limite (2.1.7) coincidem com a noção clássica de cone normal usada na análise convexa e nesse caso usamos a notação comum $N_{\Omega}\left(z^{*}\right)$. Em geral, temos a inclusão $\widehat{N}_{\Omega}(z) \subset N_{\Omega}(z)$ para qualquer $z \in \Omega$.

Teorema 2.1.1. (RW09, Teorema 6.28)

a) O cone regular normal $\widehat{N}_{\Omega}\left(z^{*}\right)$ é um cone fechado convexo. Além disso, $\widehat{N}_{\Omega}\left(z^{*}\right)=T_{\Omega}^{\circ}\left(z^{*}\right)$.

b) Defina $\widehat{T}_{\Omega}\left(z^{*}\right):=\liminf _{t \downarrow 0}\left(\Omega-z^{*}\right) / t$. Então $\widehat{T}_{\Omega}\left(z^{*}\right)=N_{\Omega}^{\circ}\left(z^{*}\right)$ sempre que $\Omega$ seja um conjunto localmente fechado em $z^{*}$. Como consequência $N_{\Omega}^{\circ}\left(z^{*}\right) \subset T_{\Omega}\left(z^{*}\right)$. 
Quando $\Omega$ é convexo, existe uma relação próxima entre o cone normal e a projeção euclidiana.

Proposição 2.1.2. (RW09, Proposição 6.17)

Seja $\Omega$ um conjunto convexo fechado e $x \in \Omega$. Então o cone normal $N_{\Omega}$ e a projeção euclidiana $P_{\Omega}$ cumprem a seguinte relação:

$$
\omega \in N_{\Omega}(x) \text { se, e somente se } P_{\Omega}(x+\omega)=x,
$$

onde $P_{\Omega}(x)$ é a única solução de problema de otimização: minimizar $\|x-\omega\|^{2}$ sujeito a $\omega \in \Omega$.

Teorema 2.1.3. Seja $x^{*} \in \Omega$ um minimo local de (2.0.3), então

$$
-\nabla f\left(x^{*}\right) \in T_{\Omega}\left(x^{*}\right)^{\circ}
$$

A expressão 2.1.9 é chamada de condição geométrica de otimalidade.

Demonstração. Seja $d \in T_{\Omega}\left(x^{*}\right)$, da definição de cone tangente, existem sequências $\left\{t_{k}\right\} \in \mathbb{R}_{+}$e $\left\{d^{k}\right\}$ tais que $t_{k} \rightarrow 0, d^{k} \rightarrow d$ e $x^{*}+t_{k} d^{k} \in \Omega$ para todo $k \in \mathbb{N}$. Desde que $x^{*}+t_{k} d^{k} \rightarrow x^{*}$ temos que $f\left(x^{*}+t_{k} d^{k}\right) \geq f\left(x^{*}\right)$ para $k$ suficientemente grande. Usando a expansão de Taylor, temos

$$
f\left(x^{*}+t_{k} d^{k}\right)=f\left(x^{*}\right)+t_{k}\left\langle\nabla f\left(x^{*}\right), d^{k}\right\rangle+o\left(t_{k}\right) \geq f\left(x^{*}\right)
$$

e como consequência

$$
t_{k}\left\langle\nabla f\left(x^{*}\right), d^{k}\right\rangle+o\left(t_{k}\right) \geq 0
$$

para $k$ suficientemente grande. Dividindo a expressão 2.1.11 por $t_{k}$ e tomando limite concluímos que $\left\langle\nabla f\left(x^{*}\right), d\right\rangle \geq 0$ para todo $d \in T_{\Omega}\left(x^{*}\right)$, isto é, $-\nabla f\left(x^{*}\right) \in T_{\Omega}\left(x^{*}\right)^{\circ}$.

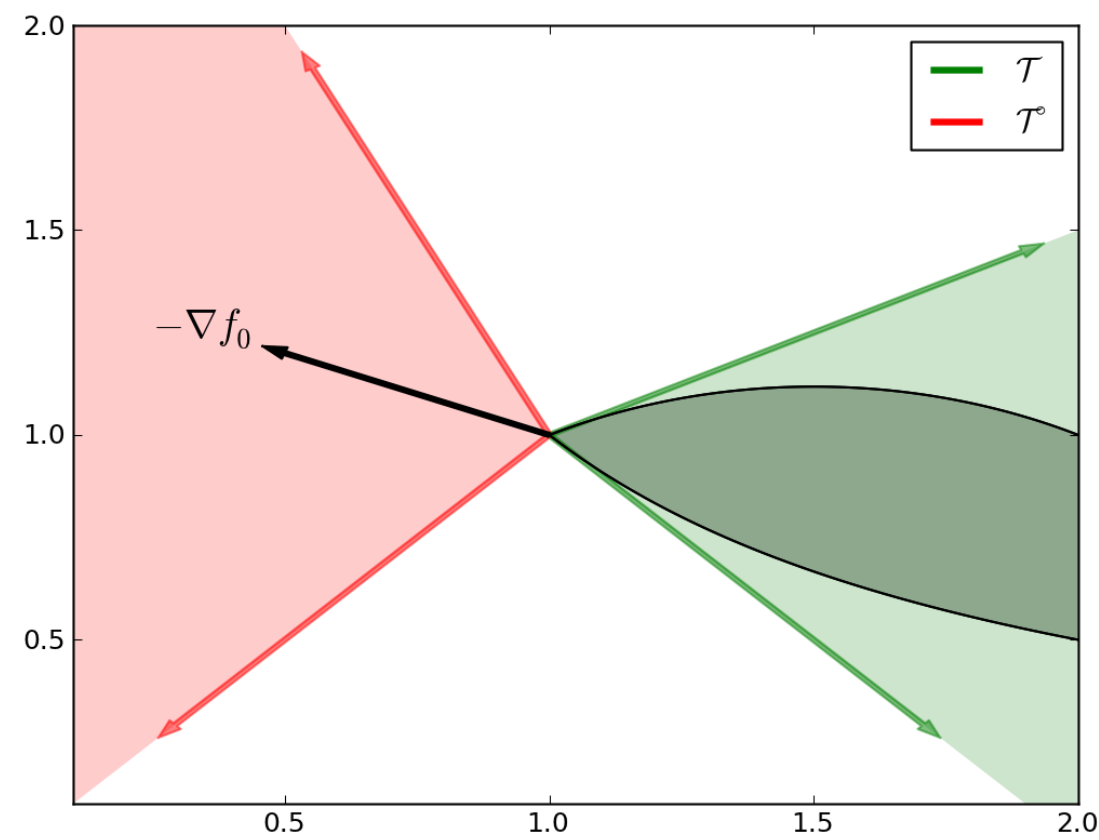

Figura 2.1: Condição Geométrica de Otimalidade de primeira ordem.

A condição geométrica 2.1.9 é uma das mais versáteis expressões para a otimalidade e tem servido como motivação para o estudo das equações generalizadas (Rob80).

Lamentavelmente, a condição 2.1.9 não é facilmente verificável. Uma das ideias para superar esse empecilho é realizar uma aproximação linear do cone tangente: 
O cone linearizado $L_{\Omega}\left(x^{*}\right)$ é definido como

$$
L_{\Omega}\left(x^{*}\right):=\left\{d \in \mathbb{R}^{n}:\left\langle\nabla h_{i}\left(x^{*}\right), d\right\rangle=0, \forall i \in\{1, \ldots, m\},\left\langle\nabla g_{j}\left(x^{*}\right), d\right\rangle \leq 0, \forall j \in J\left(x^{*}\right)\right\} .
$$

Proposição 2.1.4. A inclusão $T_{\Omega}\left(x^{*}\right) \subset L_{\Omega}\left(x^{*}\right)$ sempre se cumpre.

Demonstração. Seja $d \in T_{\Omega}\left(x^{*}\right)$, por definição, existem sequências $\left\{t_{k}\right\} \in \mathbb{R}_{+}$e $\left\{d^{k}\right\}$ tais que $t_{k} \rightarrow 0$, $d^{k} \rightarrow d$ e $x^{*}+t_{k} d^{k} \in \Omega$ para todo $k \in \mathbb{N}$. Usando a expansão de Taylor e o fato que $h_{i}\left(x^{*}\right)=0$ e $g_{j}\left(x^{*}\right)=0$ para todo $i \in\{1, \ldots, m\}, j \in J\left(x^{*}\right)$, temos

$$
\begin{array}{ll}
h_{i}\left(x^{*}+t_{k} d^{k}\right)=t_{k}\left\langle\nabla h_{i}\left(x^{*}\right), d^{k}\right\rangle+o\left(t_{k}\right)=0 & \text { para todo } i \in\{1, \ldots, m\} \\
g_{j}\left(x^{*}+t_{k} d^{k}\right)=t_{k}\left\langle\nabla g_{j}\left(x^{*}\right), d^{k}\right\rangle+o\left(t_{k}\right) \leq 0 & \text { para todo } j \in J\left(x^{*}\right) .
\end{array}
$$

Dividindo por $t_{k}$ as expressões 2.1.13) e 2.1.14) e tomando limite obtemos que $d \in L_{\Omega}\left(x^{*}\right)$

Existem casos em que a inclusão $T_{\Omega}\left(x^{*}\right) \subset L_{\Omega}\left(x^{*}\right)$ é estrita. Quando temos a igualdade $T_{\Omega}\left(x^{*}\right)=$ $L_{\Omega}\left(x^{*}\right)$ dizemos que a condição de qualificação de Abadie é satisfeita em $x^{*}$ e quando $T_{\Omega}^{\circ}\left(x^{*}\right)=$ $L_{\Omega}^{\circ}\left(x^{*}\right)$ dizemos que a condição de qualificação de Guignard vale em $x^{*}$. Claramente a condição de Abadie implica a condição de Guignard e ambas condições são suficientes para garantir a validade das condições KKT em $x^{*}$ sempre que $x^{*}$ seja um minimizador local de 2.0.3).

A condição de Guignard tem um lugar especial entre as condições de qualificação porque ela é a mínima condição de qualificação possível, no sentido que ela é implicada por qualquer outra condição de qualificação. Assim, se a condição de Guignard não vale em $x^{*}$ é porque é possível construir uma função objetivo para o problema (2.0.3) tal que $x^{*}$ é um mínimo local para tal problema mas as condições KKT não são cumpridas.

Quando as funções associadas ao problema (2.0.3) têm derivadas de ordem maior, podemos fazer uma análise de segunda ordem. Com o proposito de proceder com essa análise necessitamos uma forma para descrever a possível curvatura associada ao conjunto $\Omega$. Com esse intuito definimos o cone tangente de segunda ordem do conjunto $\Omega$ na direção $d \in T_{\Omega}\left(x^{*}\right)$, (Com90, Pen98, BS00)

$$
T_{\Omega}^{2}\left(x^{*}, d\right):=\limsup _{t \downarrow 0} \frac{\Omega-x^{*}-t d}{\frac{1}{2} t^{2}}=\left\{z \in \mathbb{R}^{m}: \exists t_{k} \downarrow 0, \exists z^{k} \rightarrow z \text { tal que } x^{*}+t_{k} d+\frac{1}{2} t_{k}^{2} z^{k} \in \Omega \forall k\right\} .
$$

O cone $T_{\Omega}^{2}\left(z^{*}, d\right)$ é um conjunto fechado não necessariamente convexo, que pode ser vazio, (BS00, Exemplo 3.29). Para evitar esse tipo de problema consideremos o cone tangente assintótico de segunda ordem do conjunto $\Omega$ na direção $d \in T_{\Omega}\left(x^{*}\right)$, (Pen98)

$$
T_{\Omega}^{2, \infty}\left(x^{*}, d\right):=\left\{z \in \mathbb{R}^{m}: \exists t_{k} \downarrow 0, \exists r_{k} \downarrow 0, \exists z^{k} \rightarrow z, r_{k}^{-1} t_{k} \rightarrow 0 \text { e } x^{*}+t_{k} d+\frac{1}{2} r_{k}^{-1} t_{k}^{2} z^{k} \in \Omega \forall k\right\} .
$$

Um resultado interessante é que apesar do cone $T_{\Omega}^{2}\left(x^{*}, d\right)$ pode ser vazio para certas (ou todas) direções $d \in T_{\Omega}\left(x^{*}\right)$, a união $T_{\Omega}^{2}\left(x^{*}, d\right) \cup T_{\Omega}^{2, \infty}\left(x^{*}, d\right)$ sempre é não vazia para qualquer direção $d \in T_{\Omega}\left(x^{*}\right)$, (Pen98, Proposição 2.1). Com esses objetos disponíveis, temos a seguinte condição de otimalidade geométrica de segunda ordem.

Teorema 2.1.5. (Pen98, Corolário 2.1)

Suponha que $x^{*} \in \Omega$ é um minimizador local de 2.0.3) então para todo $d \in T_{\Omega}\left(x^{*}\right) \cap\left\{\nabla f\left(x^{*}\right)\right\}^{\perp}$ temos:

1. $\left\langle\nabla f\left(x^{*}\right), z\right\rangle+\left\langle\nabla^{2} f\left(x^{*}\right) d, d\right\rangle \geq 0$ para todo $z \in T_{\Omega}^{2}\left(x^{*}, d\right) ;$

2. $\left\langle\nabla f\left(x^{*}\right), z\right\rangle \geq 0$ para todo $z \in T_{\Omega}^{2, \infty}\left(x^{*}, d\right)$.

O principal inconveniente é que tanto $T_{\Omega}^{2}\left(x^{*}, d\right)$ como $T_{\Omega}^{2, \infty}\left(x^{*}, d\right)$ não dependem diretamente dos dados iniciais nem são facilmente computáveis. Assim, condições adicionais sobre as restrições 
são necessárias para computar tais cones ou para re-escrever os itens 1) e 2) do Teorema (2.1.5) numa forma dual. O seguinte resultado, demonstrado para um caso mais geral, pode ser simplificado para o caso particular de $\Omega$ da forma 2.0.2.

Teorema 2.1.6. (BS00, Teorema 3.45)

Seja $x^{*} \in \Omega$ minimo local de 2.0.3). Suponha que a condição $M F C Q$ vale em $x^{*}$. Então a seguinte condição de otimalidade de segunda ordem vale

$$
\sup _{(\lambda, \mu) \in \mathcal{M}\left(x^{*}\right)}\left\langle\nabla^{2} L\left(x^{*}, \lambda, \mu\right) d, d\right\rangle \geq 0 \quad \text { para todo } d \in T_{\Omega}\left(x^{*}\right) \cap\left\{\nabla f\left(x^{*}\right)\right\}^{\perp},
$$

onde $\mathcal{M}\left(x^{*}\right)$ é o conjunto de todos os multiplicadores de Lagrange associada a (2.0.3).

\subsection{Condição de Fritz-John Generalizada}

A condição de Fritz-John é uma condição de otimalidade, simples de verificar, amplamente estudada na literatura, (Ber82, BO04, BO02, Hes75, BSS06), que não depende de nenhuma condição acerca do conjunto viável. Considere o seguinte teorema.

Teorema 2.2.1. (Ber99, Proposição 3.3.5) Seja $x^{*} \in \Omega$ um minimizador local de (2.0.3). Então existem $\mu_{0} \geq 0$ e multiplicadores $\lambda \in \mathbb{R}^{m}$ e $\mu \in \mathbb{R}_{+}^{p}$ tais que

(i) $\mu_{0} \nabla f\left(x^{*}\right)+\sum_{i=1}^{m} \lambda_{i} \nabla h_{i}\left(x^{*}\right)+\sum_{j=1}^{p} \mu_{j} \nabla g_{j}\left(x^{*}\right)=0$;

(ii) $\left(\mu_{0}, \lambda, \mu\right)$ é diferente do vetor zero;

(iii) Para qualquer vizinhança $V$ de $x^{*}$, existe um $x \in V$ tal que $\lambda_{i} h_{i}(x)>0$ se $\lambda_{i} \neq 0$ e $\mu_{j} g_{j}(x)>0$ se $\mu_{j} \neq 0$.

Quando se cumprem os itens (i) e (ii) do teorema anterior mais a condição de complementaridade 2.0.6) (i.e, $\left\langle\mu, g\left(x^{*}\right)\right\rangle=0$ ) dizemos que a condição de Fritz-John é satisfeita, e quando se cumprem os itens (i), (ii) e (iii) dizemos a que a condição de Fritz John generalizada vale em $x^{*} \in \Omega$. O item (iii) está relacionado com a noção de complementaridade. De fato a complementaridade é implicada pelo item (iii). Assim, quando $\mu_{0}$ é não nulo, temos que a condição de Fritz-John implica as condições KKT. De fato podemos pensar as condições KKT como a condição de Fritz-John mais a hipótese adicional $\mu_{0}>0$. Existe uma interessante relação entre as condições KKT, as condições de qualificação e a condição de Fritz-John: a proposição "KKT ou não MFCQ" é equivalente à condição de Fritz-Johh, onde MFCQ é a condição de Mangasarian-Fromovitz, definida na próxima seção. Ver também (NW06). A condição de Fritz-John generalizada implica a proposição "KKT ou não Quasinormalidade". Veja a Seção 2.3 para a definição de Quasinormalidade e outras condições de qualificação.

Uma desvantagem da condição de Fritz-John em relação às condições KKT, é que mesmo no caso linear, ela não é suficiente para garantir a otimalidade, como o seguinte exemplo mostra:

$$
\operatorname{minimizar} f(x)=-x_{1} \text { s.a } g_{1}(x)=x_{1}+x_{2} \leq 0, g_{2}(x)=-x_{1}-x_{2} \leq 0, g_{3}(x)=-x_{2} \leq 0 \text {. }
$$

Nesse caso, a única solução é $x^{*}:=(0,0)$ mas qualquer ponto sobre a linha $x_{1}+x_{2}=0$ com $x_{2} \geq 0$ satisfaz a condição de Fritz-John, defina $\mu_{0}, \mu_{3}=0$ e $\mu_{1}, \mu_{2}=\alpha \operatorname{com} \alpha>0$. Ver Figura 2.2 .

\subsection{Condições de Qualificação}

As condições de qualificação são ferramentas fundamentais para derivar caracterizações acerca das soluções dos problemas de programação ma temática, para a análise de estabilidade e sensibilidade, para a análise de convergência e de taxa de convergência dos métodos computacionais. 


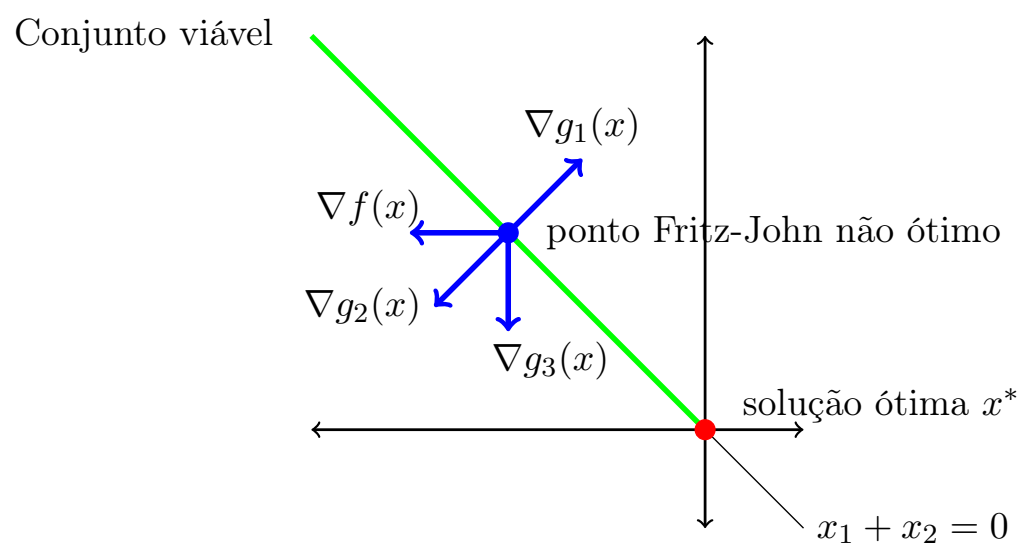

Figura 2.2: A condição de Fritz-John não é suficiente para a otimalidade, mesmo no caso linear.

As condições de qualificação são proposições CQ acerca de pontos de $\Omega$ quais dependem somente de $\Omega$ e da descrição dela, tal que a expressão

$$
\text { Minimizador local }+\mathrm{CQ} \Rightarrow \mathrm{KKT}
$$

sempre é verdadeira. Em outras palavras, sob qualquer condição de qualificação CQ, todo mínimo local de 2.0.3 cumpre com as condições KKT. Desta forma, a expressão "KKT ou não CQ" é uma condição de otimalidade. Nesta seção faremos uma pequena revisão das condições de qualificação encontradas na literatura.

Indubitavelmente, a condição de qualificação mais importante é a condição de regularidade ou de independência linear LICQ. Dizemos que a condição LICQ vale em $x^{*} \in \Omega$ se o conjunto de vetores $\left\{\nabla h_{i}\left(x^{*}\right), \nabla g_{j}\left(x^{*}\right): i \in\{1, \ldots, m\}, j \in J\left(x^{*}\right)\right\}$ é linearmente independente. A condição LICQ é robusta no sentido que ela não é só satisfeita em $x^{*}$ é também satisfeita por todo ponto viável em uma vizinhança de $x^{*}$. A popularidade se deve a duas propriedades independentes uma da outra: A primeira, é que LICQ é simples de enunciar e verificar e a segunda, é que LICQ pode ser utilizado na análise de muitos algoritmos práticos. Uma consequência importante é que, sob a condição de independência linear, existe um único multiplicador de Lagrange associado a (2.0.3). Infelizmente, a condição LICQ é muita restritiva e portanto muitos problemas importantes de programação matemática não podem ser analisados sob essa condição. Ainda mais, a condição LICQ não permite nenhuma redundância na descrição de $\Omega$, escrever uma restrição de igualdade como duas de desigualdade destrói a propriedade de independência linear. Com o intuito de enfraquecer a condição LICQ muitas outras variantes têm sido propostas na literatura.

A condição de qualificação Mangasarian-Fromovitz (MFCQ), afirma que a única solução do sistema linear

$$
\sum_{i=1}^{m} \lambda_{i} \nabla h_{i}\left(x^{*}\right)+\sum_{j \in J\left(x^{*}\right)} \mu_{j} \nabla g_{j}\left(x^{*}\right)=0, \quad \mu_{j} \geq 0 \text { para todo } j \in J\left(x^{*}\right)
$$

é a trivial. Usando o teorema da alternativa de Farkas, (RW09, Lema 6.45), a condição MFCQ é equivalente a pedir que o conjunto $\left\{h_{i}\left(x^{*}\right): i \in\{1, \ldots, m\}\right\}$ seja linearmente independente e que exista um vetor $d \in \mathbb{R}^{n}$ tal que $\left\langle\nabla h_{i}\left(x^{*}\right), d\right\rangle=0$ para todo $i \in\{1, \ldots, m\}$ e $\left\langle\nabla g_{j}\left(x^{*}\right), d\right\rangle<0$ para todo $j \in J\left(x^{*}\right)$. A condição MFCQ é mais fraca que LICQ. Além disso, ela é equivalente a dizer que o conjunto de multiplicadores de Lagrange $\mathcal{M}\left(x^{*}\right)$ é um compacto não vazio (Gau77). MFCQ, assim como LICQ, não permite redundância na descrição de $\Omega$.

A condição de qualificação de posto constante CRCQ vale em $x^{*} \in \Omega$ se existe uma vizinhança 
$V$ de $x^{*}$ tal que para todo $I \subset\{1, \ldots, m\}, J \subset J\left(x^{*}\right)$

$$
\left\{\nabla h_{i}(x), \nabla g_{j}(x): i \in I, j \in J\right\} \text { tem o mesmo posto para todo } x \in V \text {. }
$$

A condição CRCQ é independente da MFCQ, no sentido que ela não é implicada nem implica tal condição (Jan84). Ela é robusta no sentido que se vale em ponto ela também vale para todo ponto viável numa vizinhança desse ponto. Uma diferença fundamental com respeito às condições anteriores é que ela permite redundância na descrição de $\Omega$. Ainda mais, quando as restrições são afins, CRCQ sempre vale. A condição CRCQ é uma condição de segunda ordem, isto é, ela pode ser usada como CQ2 na Proposição (2.0.14), (AES10), da mesma forma que a condição LICQ, enquanto a condição MFCQ não, (Aru91, Ani00).

Dizemos que a Condição de Dependência Linear Positiva Constante CPLD é satisfeita no ponto $x^{*} \in \Omega$ se existe uma vizinhança $V$ de $x^{*}$ tal que sempre que o conjunto $\left\{\nabla h_{i}\left(x^{*}\right), \nabla g_{j}\left(x^{*}\right): i \in\right.$ $I, j \in J\}$ seja positivo-linearmente dependente para certos conjuntos de índices $I \subset\{1, \ldots, m\}$ e $J \subset J\left(x^{*}\right)$ implica que

$$
\left\{\nabla h_{i}(x), \nabla g_{j}(x): i \in I, j \in J\right\} \text { é positivo-linearmente para todo } x \in V \text {. }
$$

A condição CPLD é estritamente mais fraca que a condição MFCQ e a condição CRCQ. Além disso a condição CPLD tem sido usada na análise de convergência de vários métodos numéricos, (BM14, QW00). Motivados pela busca de condições de qualificação fracas, versões relaxadas das condições CRCQ e CPLD foram propostas.

A condição de qualificação de posto constante relaxada RCRCQ, (MS11), requer a existência de uma vizinhança $V$ de $x^{*}$ tal que para todo subconjunto $J \subset J\left(x^{*}\right)$,

$$
\left\{\nabla h_{i}(x), \nabla g_{j}(x): i \in\{1, \ldots, m\}, j \in J\right\} \text { tem o mesmo posto para todo } x \in V \text {. }
$$

A condição de dependência linear positiva constante relaxada RCPLD, (AHSS12a), vale no ponto viável $x^{*}$ se existe uma vizinhança $V$ de $x^{*}$ tal que (i) $\left\{\nabla h_{i}(x): i \in\{1, \ldots, m\}\right\}$ tem posto constante para todo $x \in V$ e (ii) se existe um conjunto $J \subset J\left(x^{*}\right)$ tal que $\left\{\nabla h_{i}\left(x^{*}\right), \nabla g_{j}\left(x^{*}\right): i \in I, j \in J\right\}$ é positivo-linearmente dependente então

$$
\left\{\nabla h_{i}(x), \nabla g_{j}(x): i \in I, j \in J\right\} \text { é positivo-linearmente dependente para } x \in V,
$$

onde $I$ é qualquer subconjunto de índices de $\{1, \ldots, m\}$ tal que $\left\{\nabla h_{i}(x): i \in I\right\}$ forma uma base linear para o subespaço gerado por $\left\{\nabla h_{i}(x): i \in\{1, \ldots, m\}\right\}$. A definição de RCPLD independe da escolha de $I$ (AHSS12a) $)$ A condição RCPLD é mais forte que a condição de Abadie, implica a condição de Error Bound e ela é mais fraca que CPLD e que RCRCQ. RCPLD vale sempre que as restrições sejam dadas por funções afins.

Para continuar com a revisão, precisamos das seguintes definições. O intuito é fornecer uma decomposição natural do cone polar $L_{\Omega}^{\circ}\left(x^{*}\right)$. Seja $J_{-}$o subconjunto de índices $\ell \in J\left(x^{*}\right)$ tal que, para todo $\ell \in J_{-}$, existam $\lambda_{1}, \ldots, \lambda_{m} \in \mathbb{R}$ e $\mu_{j} \in \mathbb{R}_{+}$para $j \in J\left(x^{*}\right)$, tais que

$$
-\nabla g_{\ell}\left(x^{*}\right)=\sum_{i=1}^{m} \lambda_{i} \nabla h_{i}\left(x^{*}\right)+\sum_{j \in J\left(x^{*}\right)} \mu_{j} \nabla g_{j}\left(x^{*}\right) .
$$

Finalmente, $J_{+}:=J\left(x^{*}\right) \backslash J_{-}$. Ambos subconjuntos de índices $J_{-}$e $J_{+}$dependem explicitamente do ponto $x^{*}$, assim o razoável é escrever $J_{-}\left(x^{*}\right)$ e $J_{+}\left(x^{*}\right)$, mas quando é claro do contexto simplesmente escrevemos $J_{-}$e $J_{+}$.

A condição de posto constante da componente subespaço CRSC é satisfeita em $x^{*}$ se existe uma vizinhança $V$ de $x^{*}$ tal que

$$
\left\{\nabla h_{i}(x), \nabla g_{j}(x): i \in\{1, \ldots, m\}, j \in J_{-}\right\} \text {tem posto constante para todo } x \in V \text {. }
$$


Esta condição foi introduzida por Andreani, Haeser, Schuverdt e Silva em (AHSS12b). Note que o subespaço linear gerado pelos vetores $\left\{\nabla h_{i}\left(x^{*}\right), \nabla g_{j}\left(x^{*}\right): i \in\{1, \ldots, m\}, j \in J_{-}\right\}$é o maior subespaço linear incluso em $L_{\Omega}^{\circ}\left(x^{*}\right)$ e o cone gerado por $\left\{\nabla g_{j}\left(x^{*}\right): j \in J_{+}\right\}$é o maior cone pontudo (i.e cone que não contém subespaços de dimensão um) contido em $L_{\Omega}^{\circ}\left(x^{*}\right)$. A condição CRSC possui a propriedade de Error Bound, implica a condição de Abadie e é implicada por RCPLD.

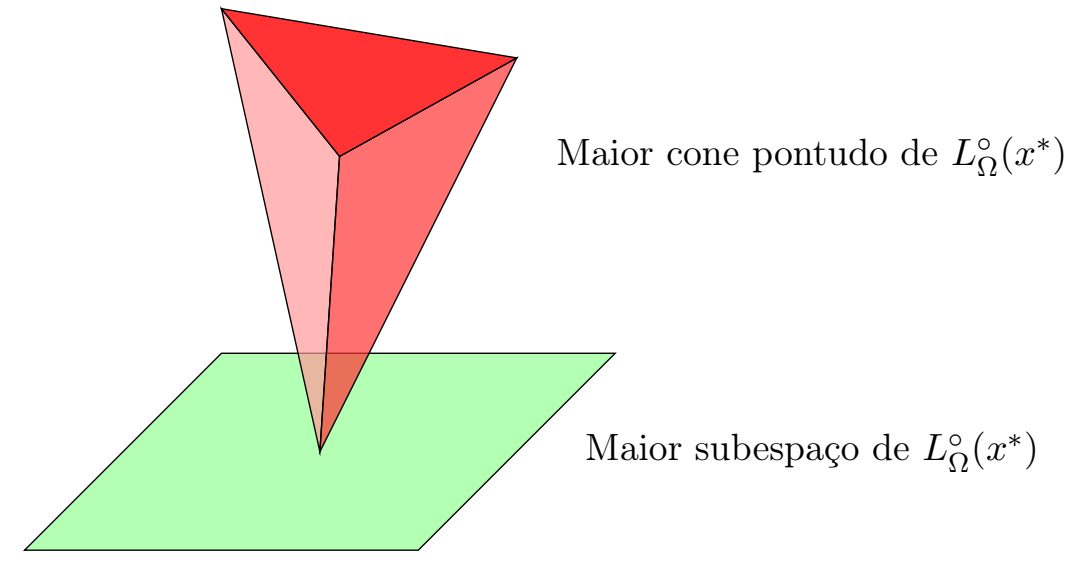

Figura 2.3: Decomposição de um cone em um subespaço e em um cone pontudo.

A condição de qualificação CPG, da siglas em inglês para Constant Positive Generator vale em $x^{*}$ se existem subconjuntos (possivelmente vazios) $I^{\prime} \subset\{1, \ldots, m\}$ e $J^{\prime} \subset J_{-}$e uma vizinhança $V$ de $x^{*}$ tais que

(i) Os gradientes $\nabla h_{i}\left(x^{*}\right)$ e $\nabla g_{j}\left(x^{*}\right)$ indexada por $i \in I^{\prime}$ e $j \in J^{\prime}$ são linearmente independentes;

(ii) Para todo $x \in V$, se

$$
z=\sum_{i=1}^{m} \lambda_{i}^{\prime} \nabla h_{i}(x)+\sum_{j \in J\left(x^{*}\right)} \mu_{j}^{\prime} \nabla g_{j}(x)
$$

com $\mu_{j}^{\prime} \geq 0$ para todo $j \in J\left(x^{*}\right)$, então para todo $i \in I^{\prime}, \ell \in J^{\prime}$, e $j \in J_{+}$, existem $\lambda_{i}^{\prime \prime} \in \mathbb{R}$, $\lambda_{\ell}^{\prime \prime \prime} \in \mathbb{R}$, e $\mu_{j}^{\prime \prime} \in \mathbb{R}_{+}$tais que

$$
z=\sum_{i \in I^{\prime}} \lambda_{i}^{\prime \prime} \nabla h_{i}(x)+\sum_{\ell \in J^{\prime}} \lambda_{\ell}^{\prime \prime \prime} \nabla g_{\ell}(x)+\sum_{j \in J_{+}} \mu_{j}^{\prime \prime} \nabla g_{j}(x)
$$

A condição CPG depende explicitamente dos subconjuntos de índices $I^{\prime} \subset\{1, \ldots, m\}$ e $J^{\prime} \subset J_{-}$, isto é, o item (ii) pode não ser satisfeito para outros subconjuntos que cumprem o item (i). A condição CPG é implicada pela condição CRSC e implica a condição de Abadie (AHSS12b).

Observação 2.3.1. Devido à definição de $J_{-}$, o conjunto $\left\{\nabla h_{i}\left(x^{*}\right), \nabla g_{j}\left(x^{*}\right): i \in I^{\prime}, j \in J^{\prime}\right\}$ é linearmente independente se, e somente se, $\left\{\nabla h_{i}\left(x^{*}\right), \nabla g_{\ell}\left(x^{*}\right), \nabla g_{j}\left(x^{*}\right): i \in I^{\prime}, j \in J^{\prime}, j \in J_{+}\right\}$é positivo-linearmente independente, isto é, a única solução de

$$
\sum_{i \in I^{\prime}} \lambda_{i} \nabla h_{i}\left(x^{*}\right)+\sum_{\ell \in J^{\prime}} \gamma_{\ell} \nabla g_{\ell}\left(x^{*}\right)+\sum_{j \in J_{+}} \mu_{j} \nabla g_{j}\left(x^{*}\right)=0
$$

onde $\lambda_{i} \in \mathbb{R}, i \in I^{\prime}, \gamma_{\ell} \in \mathbb{R}, \ell \in J^{\prime}$ e $\mu_{j} \geq 0, j \in J_{+}$é a trivial.

Associado aos subconjuntos de índices $I^{\prime} \subset\{1, \ldots, m\}, J^{\prime} \subset J_{-}$e $J_{+}=J\left(x^{*}\right) \backslash J-$, temos o cone

$$
K_{I^{\prime}, J^{\prime}}(x):=\left\{\sum_{i \in I^{\prime}} \lambda_{i} \nabla h_{i}(x)+\sum_{\ell \in J^{\prime}} \gamma_{\ell} \nabla g_{\ell}(x)+\sum_{j \in J_{+}} \mu_{j} \nabla g_{j}(x): \mu_{j} \in \mathbb{R}_{+}, \lambda_{i}, \gamma_{\ell} \in \mathbb{R}\right\} .
$$


Lema 2.3.1. Seja $x^{*} \in \Omega$ um ponto viável e sejam $I^{\prime} \subset\{1, \ldots, m\}, J^{\prime} \subset J_{-}$e $J_{+}=J\left(x^{*}\right) \backslash J_{-}$ subconjuntos de indices tais que $\left\{\nabla h_{i}\left(x^{*}\right), \nabla g_{j}\left(x^{*}\right): i \in I^{\prime}, j \in J^{\prime}\right\}$ é linearmente independente. Então a multifunção $\mathbb{R}^{n} \ni x \rightrightarrows K_{I^{\prime}, J^{\prime}}(x)$ é semicontínua exteriormente em $x^{*}$.

Demonstração. Seja $\omega^{*}$ um elemento de $\lim \sup _{x \rightarrow x^{*}} K_{I^{\prime}, J^{\prime}}(x)$, então existem sequências $\left\{x^{k}\right\}$ e $\left\{\omega^{k}\right\}$ tais que $x^{k} \rightarrow x^{*}, \omega^{k} \rightarrow \omega^{*}$ e $\omega^{k} \in K_{I^{\prime}, J^{\prime}}\left(x^{k}\right)$, onde

$$
\omega^{k}=\sum_{i \in I^{\prime}} \lambda_{i}^{k} \nabla h_{i}\left(x^{k}\right)+\sum_{\ell \in J^{\prime}} \gamma_{\ell}^{k} \nabla g_{\ell}\left(x^{k}\right)+\sum_{j \in J_{+}} \mu_{j}^{k} \nabla g_{j}\left(x^{k}\right)
$$

para certas sequências $\left\{\lambda_{i}^{k} \in \mathbb{R}, i \in I^{\prime}\right\},\left\{\gamma_{\ell}^{k} \in \mathbb{R}, \ell \in J^{\prime}\right\}$ e $\left\{\mu_{j}^{k} \in \mathbb{R}_{+}, j \in J_{+}\right\}$. Agora, defina $M_{k}:=\max \left\{\left|\lambda_{i}^{k}\right|, i \in I^{\prime} ;\left|\gamma_{\ell}^{k}\right|, \ell \in J^{\prime} ; \mu_{j}^{k}, j \in J_{+}\right\}$. Temos as seguintes duas possibilidades:

(i) $\left\{M_{k}\right\}$ admite alguma subsequência limitada. Podemos considerar, possivelmente depois de extrair uma subsequência adequada, que para todo $i \in I^{\prime}, \ell \in J^{\prime}$ e $j \in J_{+}$as sequências $\lambda_{i}^{k}$, $\gamma_{\ell}^{k}$ e $\mu_{j}^{k}$ têm como limites $\lambda_{i}^{*}, \gamma_{\ell}^{*}$ e $\mu_{j}^{*}$ respectivamente. Tomando limite na expressão 2.3.10 obtemos

$$
\omega^{*}=\sum_{i \in I^{\prime}} \lambda_{i}^{*} \nabla h_{i}\left(x^{*}\right)+\sum_{\ell \in J^{\prime}} \gamma_{\ell}^{*} \nabla g_{\ell}\left(x^{*}\right)+\sum_{j \in J_{+}} \mu_{j}^{*} \nabla g_{j}\left(x^{*}\right) \in K_{I^{\prime}, J^{\prime}}\left(x^{*}\right) .
$$

(ii) Caso contrário, temos $M_{k} \rightarrow \infty$. Dividindo 2.3 .10 por $M_{k}$, chegamos a

$$
\frac{\omega^{k}}{M_{k}}=\sum_{i \in I^{\prime}} \frac{\lambda_{i}^{k}}{M_{k}} \nabla h_{i}\left(x^{k}\right)+\sum_{l \in J^{\prime}} \frac{\gamma_{\ell}^{k}}{M_{k}} \nabla g_{\ell}\left(x^{k}\right)+\sum_{j \in J_{+}} \frac{\mu_{j}^{k}}{M_{k}} \nabla g_{j}\left(x^{k}\right) .
$$

Desde que $\max \left\{\left|\lambda_{i}^{k} / M_{k}\right|, i \in I^{\prime} ;\left|\gamma_{\ell}^{k} / M_{k}\right|, \ell \in J^{\prime} ; \mu_{j}^{k} / M_{k}, j \in J_{+}\right\}=1$ para todo $k \in \mathbb{N}$, podemos extrair uma subsequência convergente. Por conseguinte, tomando limites em (2.3.11), obtemos uma contradição com o fato que $\left\{\nabla h_{i}\left(x^{*}\right), \nabla g_{\ell}\left(x^{*}\right), \nabla g_{j}\left(x^{*}\right): i \in I^{\prime}, \ell \in J^{\prime}, j \in J_{+}\right\}$ é um conjunto positivamente independente, ver Observação 2.3.1.

Para terminar com esta subseção, mencionamos mais duas condições de qualificação. A condição de qualificação de Quasinormalidade vale em $x^{*} \in \Omega$ se sempre que $\sum_{j=1}^{m} \lambda_{j} \nabla h_{j}\left(x^{*}\right)+$ $\sum_{j \in J\left(x^{*}\right)} \mu_{j} \nabla g_{j}\left(x^{*}\right)=0$ para certos $\lambda \in \mathbb{R}^{m}$ e $\mu_{j} \in \mathbb{R}_{+} \forall j \in J\left(x^{*}\right)$, não existe sequência $\left\{x^{k}\right\}$ com $x^{k} \rightarrow x^{*}$ tal que para todo $k \in \mathbb{N}, \lambda_{i} h_{i}\left(x^{k}\right)>0$ quando $\lambda_{i}$ é não zero e $\mu_{j} g_{j}\left(x^{k}\right)>0$ quando $\mu_{j}>0$, (Hes75, Ber99, BO04).

A condição de qualificação de Pseudonormalidade vale em $x^{*} \in \Omega$ se sempre que $\sum_{j=1}^{m} \lambda_{j} \nabla h_{j}\left(x^{*}\right)+$ $\sum_{j \in J\left(x^{*}\right)} \mu_{j} \nabla g_{j}\left(x^{*}\right)=0$ para certos $\lambda \in \mathbb{R}^{m}$ e $\mu_{j} \in \mathbb{R}_{+} \forall j \in J\left(x^{*}\right)$, não existe sequência $x^{k} \rightarrow x^{*}$ tal que $\sum_{i=1}^{m} \lambda_{j} h_{j}\left(x^{k}\right)+\sum_{j \in J\left(x^{*}\right)} \mu_{j} g_{j}\left(x^{k}\right)>0$ para todo $k \in \mathbb{N}$, (Ber99, BO04, BO02).

A Pseudonormalidade é condição mais forte que Quasinormalidade e ambas condições de qualificação são estritamente mais fortes que a condição de Abadie. É interessante notar que ambas condições implicam a existência de uma penalidade exata para o problema $(2.0 .3)$, (BO02, Proposição 4.2, Proposição 4.3).

Um resumo das relações entre as condições de qualificação mencionadas está na Figura 2.4 .

\subsection{Condições Sequenciais de Otimalidade}

Métodos numéricos práticos para resolver problemas de otimização não linear são iterativos. Em cada iteração devemos decidir se é conveniente terminar com a execução do algoritmo ou não. Já que testar a otimalidade é muito difícil, a ideia é terminar com a execução quando alguma condição de otimalidade é aproximadamente satisfeita. As condições sequenciais de otimalidade servem para 


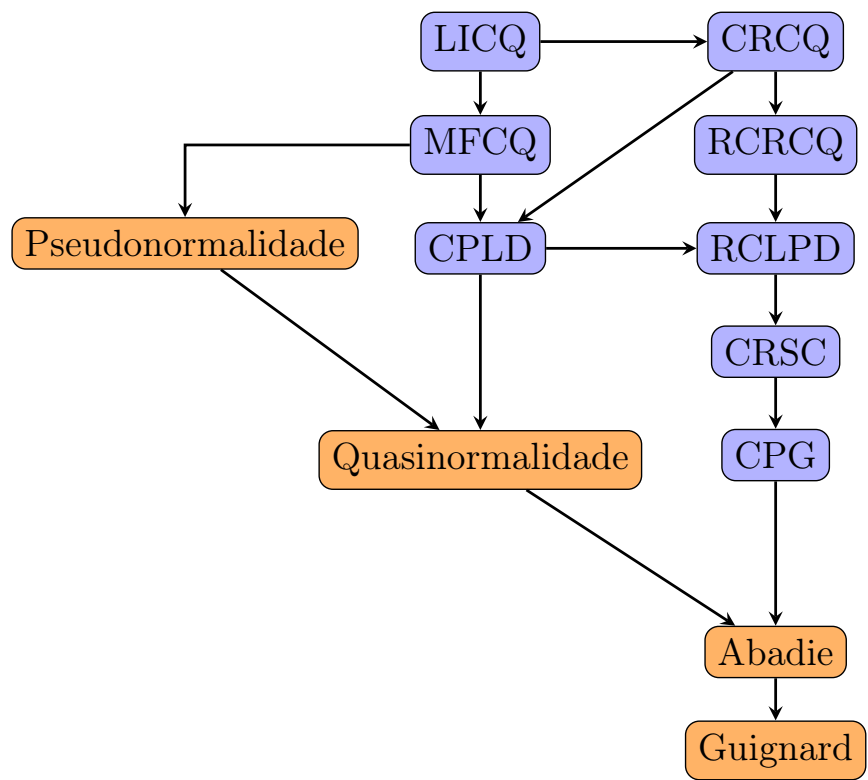

Figura 2.4: Relações entre as condições de qualificação mencionadas. Como mostraremos na tese, as condições de qualificação de Pseudonormalidade, Quasinormalidade e de Abadie não são suficientes para garantir a convergência de certos métodos numéricos.

justificar esse tipo de prática. Formalmente, dizemos que um ponto $x^{*}$ satisfaz uma Condição Sequencial de Otimalidade definida pela proposição matemática $\mathcal{P}$ quando existe uma sequência $\left\{x^{k}\right\}$ que converge a $x^{*}$ e satisfaz $\mathcal{P}\left(\left\{x^{k}\right\}\right)$. Usualmente, a condição sequencial de otimalidade está associada a certa quantidade $\varepsilon_{k}$ que deve tender a zero. O critério de parada natural que corresponde à condição sequencial de otimalidade é de parar a execução do algoritmo quando a quantidade $\varepsilon_{k}$ é suficientemente pequena.

As condições sequenciais de otimalidade devem satisfazer três requerimentos: (i) Devem ser satisfeitas por todo minimizador, independente de qualquer condição de qualificação, (ii) devem ser o mais fortes possível no sentido (2.0.7), no caso que usem informação de primeira ordem e, finalmente, (iii) devem ser possíveis de verificar por sequências geradas por algoritmos práticos.

\subsubsection{Condição sequencial AKKT}

Como dissemos na introdução, a maioria dos métodos práticos são iterativos, e decidimos terminar com a execução do algoritmo quando alguma condição de otimalidade é aproximadamente satisfeita. Apesar das condições KKT não serem, na realidade, uma condição de otimalidade, a ideia natural é de parar o algoritmo quando as condições KKT são satisfeitas aproximadamente. Esta prática computacional pode ser justificada por uma propriedade de caráter teórico dos minimizadores locais: todo minimizador local pode ser aproximado por uma sequência de pontos aproximadamente KKT.

Essa é a origem da mais popular das condições sequenciais: a condição AKKT.

Definição 2.4.1. Seja $x^{*} \in \Omega$ um ponto viável. Dizemos que a condição AKKT vale em $x^{*}$ se existem sequências $\left\{x^{k}\right\} \subset \mathbb{R}^{n},\left\{\lambda^{k}\right\} \subset \mathbb{R}^{m}$ e $\left\{\mu^{k}\right\} \subset \mathbb{R}_{+}^{p}$ com $\mu_{j}^{k}=0$ para todo $j \notin J\left(x^{*}\right)$ tais que $x^{k} \rightarrow x^{*} e$

$$
\lim _{k \rightarrow \infty} \nabla f\left(x^{k}\right)+\sum_{i=1}^{m} \lambda_{i}^{k} \nabla h_{i}\left(x^{k}\right)+\sum_{j \in J\left(x^{*}\right)} \mu_{j}^{k} \nabla g_{j}\left(x^{k}\right)=0
$$

A sequência $\left\{x^{k}\right\}$ da Definição 2.4.1 é chamada de sequência AKKT associada a $x^{*}$. A condição AKKT é de fato uma condição de otimalidade e sua validade não depende de nenhuma condição de qualificação. 
Teorema 2.4.1. Se $x^{*}$ é um minimizador local para o problema 2.0.3) então a condição AKKT vale em $x^{*}$.

Demonstração. Seja $x^{*}$ um minimizador local de 2.0.3) e $\left\{\rho_{k}\right\}$ uma sequência de números positivos tal que $\rho_{k} \rightarrow \infty$. Da definição de mínimo local, existe $\varepsilon>0$ tal que $f\left(x^{*}\right) \leq f(x)$ para todo ponto viável $x$ tal que $\left\|x-x^{*}\right\| \leq \varepsilon$. Assim o mínimo local $x^{*}$ é a única solução do seguinte problema

$$
\text { minimizar } f(x)+\frac{1}{2}\left\|x-x^{*}\right\|^{2} \text { sujeito a } h(x)=0, \quad g(x) \leq 0, \quad x \in \mathbb{B}\left(x^{*}, \varepsilon\right) .
$$

Usaremos a técnica de penalidade para 2.4.2). Para cada $\rho_{k}$, consideremos o subproblema:

$$
\text { minimizar } f(x)+\frac{1}{2}\left\|x-x^{*}\right\|^{2}+\frac{1}{2} \rho_{k}\left(\sum_{i=1}^{m} h_{i}(x)^{2}+\sum_{j=1}^{p} \max \left\{0, g_{j}(x)\right\}^{2}\right) \text { s.a } x \in \mathbb{B}\left(x^{*}, \varepsilon\right)
$$

Seja $x^{k}$ a solução do subproblema 2.4.3). A existência de $x^{k}$ é garantida pela continuidade da função objetivo de 2.4.3) e a compacidade de $\mathbb{B}\left(x^{*}, \varepsilon\right)$ (Teorema de Weierstrass). Vamos provar que a sequência $\left\{x^{k}\right\}$ converge a $x^{*}$. De fato, seja $x^{\infty}$ qualquer ponto limite de $\left\{x^{k}\right\}$. Como $x^{*}$ é viável e $x^{k}$ é um minimizador de 2.4.3, temos que

$$
\sum_{i=1}^{m} h_{i}\left(x^{k}\right)^{2}+\sum_{j=1}^{p} \max \left\{0, g_{j}\left(x^{k}\right)\right\}^{2} \leq \frac{1}{\rho_{k}}\left(f\left(x^{*}\right)-f\left(x^{k}\right)-\frac{1}{2}\left\|x^{k}-x^{*}\right\|^{2}\right)
$$

mas o lado direito de (2.4.4) vai para zero quando $k$ vai para o infinito, assim concluímos que $x^{\infty}$ dever ser viável. Usando de novo o fato que $x^{*}$ é viável e $x^{k}$ é um minimizador local de 2.4 .3 temos $f\left(x^{k}\right)+\frac{1}{2}\left\|x^{k}-x^{*}\right\|^{2} \leq f\left(x^{*}\right)$ e tomando o limite adequado, $f\left(x^{\infty}\right)+\frac{1}{2}\left\|x^{\infty}-x^{*}\right\|^{2} \leq f\left(x^{*}\right)$ mas já sabemos que $x^{\infty}$ é viável e $\left\|x^{\infty}-x^{*}\right\| \leq \varepsilon$ portanto $f\left(x^{\infty}\right)+\frac{1}{2}\left\|x^{\infty}-x^{*}\right\|^{2} \leq f\left(x^{*}\right) \leq f\left(x^{\infty}\right)$ o que implica que $x^{\infty}=x^{*}$.

Assim, para $k$ suficientemente grande, $x^{k}$ pertence ao interior de $\mathbb{B}\left(x^{*}, \varepsilon\right)$. Desta forma, temos que o gradiente da função objetivo de (2.4.3) deve ser zero em $x=x^{*}$ :

$$
\nabla f\left(x^{k}\right)+\sum_{i=1}^{m} \rho_{k} h_{i}\left(x^{k}\right) \nabla h_{i}\left(x^{k}\right)+\sum_{j=1}^{p} \rho_{k} \max \left\{0, g_{j}\left(x^{k}\right)\right\} \nabla g_{j}\left(x^{k}\right)+\left\|x^{k}-x^{*}\right\|\left(x^{k}-x^{*}\right)=0
$$

Defina $\lambda_{i}^{k}:=\rho_{k} h_{i}\left(x^{k}\right)$ para $i \in\{1, \ldots, m\}, \mu_{j}^{k}:=\rho_{k} \max \left\{0, g_{j}\left(x^{k}\right)\right\}$ para $j \in\{1, \ldots, p\}$ e $\delta_{k}:=\left\|x^{k}-x^{*}\right\|\left(x^{k}-x^{*}\right)$. Claramente, $\delta_{k} \rightarrow 0$ quando $k$ vai para o infinito e para $k$ suficientemente grande $\mu_{j}^{k}=0$ para todo $j \notin J\left(x^{*}\right)$. Com essas escolhas e tomando limite em 2.4.5 temos que 2.4.1) é satisfeita e como consequência a afirmação é provada.

O Teorema 2.4 .2 mostra como a condição AKKT é verificada na prática.

Teorema 2.4.2. (Hae09, Proposição 2.5) Um ponto $x^{*} \in \mathbb{R}^{n}$ satisfaz a condição AKKT se, e somente se, existem sequências $\left\{x^{k}\right\} \subset \mathbb{R}^{n},\left\{\lambda^{k}\right\} \subset \mathbb{R}^{m},\left\{\mu^{k}\right\} \subset \mathbb{R}_{+}^{p},\left\{\varepsilon_{\text {feas }}^{k}\right\} \subset \mathbb{R}_{+},\left\{\varepsilon_{\text {feas }}^{k}\right\} \subset \mathbb{R}_{+}$ $e\left\{\varepsilon_{\text {comp }}^{k}\right\} \subset \mathbb{R}_{+}$tais que $x^{k} \rightarrow x^{*}, \varepsilon_{\text {opt }}^{k} \rightarrow 0, \varepsilon_{\text {feas }}^{k} \rightarrow 0, \varepsilon_{\text {comp }}^{k} \rightarrow 0 e$

$$
\begin{array}{ccl}
\left\|\nabla f\left(x^{k}\right)+\sum_{i=1}^{m} \lambda_{i}^{k} \nabla h_{i}\left(x^{k}\right)+\sum_{j=1}^{p} \mu_{j}^{k} \nabla g_{j}\left(x^{k}\right)\right\| \leq \varepsilon_{\text {opt }}^{k} & \text { (otimalidade) } \\
\left\|h\left(x^{k}\right)\right\| \leq \varepsilon_{\text {feas }}^{k} \quad \text { e }\left\|\max \left\{0, g\left(x^{k}\right)\right\}\right\| \leq \varepsilon_{\text {feas }}^{k} & \text { (viabilidade) } \\
g_{j}\left(x^{k}\right)<-\varepsilon_{\text {comp }}^{k} \text { implica } \quad \mu_{j}^{k}=0 & \text { (complementaridade) }
\end{array}
$$

Demonstração. Primeiro assuma que a condição AKKT vale em $x^{*}$. Pela própria definição, $x^{*}$ deve 
ser viável. Agora defina $\varepsilon^{k}$ como

$\max \left\{\left\|h\left(x^{k}\right)\right\|,\left\|\max \left\{0, g\left(x^{k}\right)\right\}\right\|,\left\{-g_{j}\left(x^{k}\right)\right\}_{j \in J\left(x^{*}\right)},\left\|\nabla f\left(x^{k}\right)+\sum_{i=1}^{m} \lambda_{i}^{k} \nabla h_{i}\left(x^{k}\right)+\sum_{j \in J\left(x^{*}\right)} \mu_{j}^{k} \nabla g_{j}\left(x^{k}\right)\right\|\right\}$.

Da continuidade das funções $h$ e $g$ e da expressão 2.4.1 temos que $\varepsilon^{k} \rightarrow 0$. Escolha $\varepsilon_{o p t}^{k}, \varepsilon_{\text {feas }}^{k} \mathrm{e}$ $\varepsilon_{\text {comp }}^{k}$ todos iguais a $\varepsilon^{k}$ para todo $k \in \mathbb{N}$. Certamente com essas escolhas temos que 2.4.6 e 2.4.7) são satisfeitas. Somente falta provar 2.4.8). Suponha que $g_{j}\left(x^{k}\right)<-\varepsilon^{k}$ para algum $j \in\{1, \ldots, p\}$. Se $j \in J\left(x^{*}\right)$ temos que $-g_{j}\left(x^{k}\right)>\varepsilon^{k}$, o que é uma contradição com a definição de $\varepsilon^{k}$, assim $g_{j}\left(x^{k}\right)<-\varepsilon^{k}$ para todo $j \notin J\left(x^{*}\right)$ e implica que $\mu_{j}^{k}=0$.

Agora assuma que (2.4.6), 2.4.7) e (2.4.8) são satisfeitas. Tomando limite em (2.4.7) temos que $x^{*}$ é viável. De 2.4.8 temos que para todo $j \notin J\left(x^{*}\right), \mu_{j}^{k}=0$ para $k$ suficientemente grande. Assim dessas observaçoes, (2.4.6) implica (2.4.1).

Assim, o critério de parada natural associado à condição AKKT é declarar "convergência" em $x=x^{k}$ quando

$$
\begin{gathered}
\left\|\nabla f\left(x^{k}\right)+\sum_{i=1}^{m} \lambda_{i}^{k} \nabla h_{i}\left(x^{k}\right)+\sum_{j=1}^{p} \mu_{j}^{k} \nabla g_{j}\left(x^{k}\right)\right\| \leq \varepsilon_{\text {opt }} \\
\left\|h\left(x^{k}\right)\right\| \leq \varepsilon_{\text {feas }} \quad \text { e } \quad\left\|\max \left\{0, g\left(x^{k}\right)\right\}\right\| \leq \varepsilon_{\text {feas }} \\
\mu_{j}^{k}=0 \quad \text { sempre que } \quad g_{j}\left(x^{k}\right)<-\varepsilon_{\text {comp }}
\end{gathered}
$$

para escolhas de tolerâncias $\varepsilon_{o p t}, \varepsilon_{\text {comp }}$ e $\varepsilon_{\text {feas }}$ definidas previamente pelo usuário.

A popularidade da condição AKKT não se deve somente à sua simplicidade, deve-se também à sua associação com muitos algoritmos práticos, como por exemplo, o método de lagrangiano aumentado (ABMS08, BM14), alguns métodos de programação quadrática sequencial (QW00), alguns métodos de pontos interiores (G06) e métodos de restauração inexata (ㅍ00) e também à capacidade de provar convergência global desses métodos sob condições de qualificação fracas. Por outro lado, existem outros algoritmos práticos que não geram sequências AKKT, como o caso do método SQP puro (i.e. o método de Newton-Lagrange), (AMSS14).

A condição AKKT é uma condição forte porque implica (2.0.7), para condições de qualificação mais fracas que a LICQ, como por exemplo temos as condições CPLD, CRSC e a condição fraca CPG. Desta forma "KKT ou não CPG" fornece uma medida de força da condição AKKT como critério de parada. Acreditava-se que CPG era a condição de qualificação mais fraca possível que garante que AKKT implica KKT, entretanto, isso não é verdade. No capítulo 3 mostraremos que a condição de qualificação CCP (Cone Continuity Property) desempenha esse papel. Assim "KKT ou não CCP" é a medida mais exata da força de (2.4.6), 2.4.7) e 2.4.8) como critério de parada.

\subsubsection{Condição sequencial AGP}

Na procura por condições de otimalidade que se encaixem bem ao comportamento de métodos práticos, Martínez e Svaiter, em (MS03), introduziram uma nova condição de otimalidade que, a grosso modo, diz que, aproximadamente, os gradientes projetados tendem para zero. Esta condição é chamada de AGP (Approximate Gradient Projection). A condição AGP é uma condição de otimalidade que se encaixa naturalmente como critério de parada para os métodos de restauração inexata, (Mar01, MP00, FF10, BHM14, BBM15).

Definição 2.4.2. Dados um número não positivo $\gamma \in[-\infty, 0]$ e um ponto viável $x^{*} \in \Omega$, dizemos que $x^{*}$ satisfaz a condição $A G P(\gamma)$ para o problema 2.0.3) se existe uma sequência $\left\{x^{k}\right\}$ com limite $x^{*}$ tal que

$$
P_{\Omega\left(x^{k}, \gamma\right)}\left(x^{k}-\nabla f\left(x^{k}\right)\right)-x^{k} \rightarrow 0,
$$


onde $P_{\Omega\left(x^{k}, \gamma\right)}$ é a projeção ortogonal sobre o conjunto convexo fechado $\Omega\left(x^{k}, \gamma\right)$ definido como

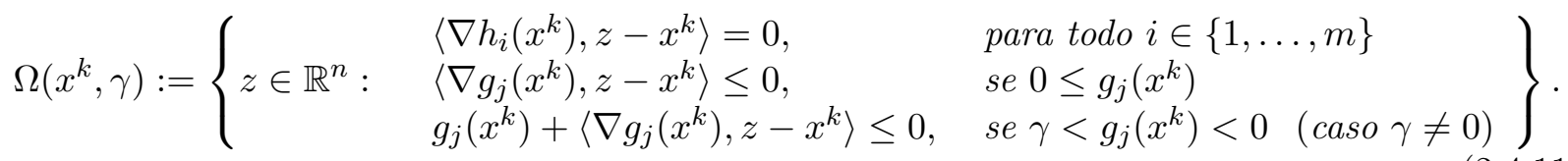

A sequência $\left\{x^{k}\right\}$ é chamada de sequência $\operatorname{AGP}(\gamma)$ associada a $x^{*}$. O parâmetro $\gamma$ serve para quantificar o grau de "profundeza" ou viabilidade permitida para a sequência $\left\{x^{k}\right\}$. A validade da condição $\operatorname{AGP}(\gamma)$ é independente do parâmetro $\gamma$ sempre que $\gamma \in[-\infty, 0)$, isto é, se a condição $\operatorname{AGP}(\gamma)$ vale em $x^{*} \in \Omega$ para algum $\gamma \in[-\infty, 0)$ então $\operatorname{AGP}\left(\gamma^{\prime}\right)$ também vale em $x^{*}$ para qualquer $\gamma^{\prime} \in[-\infty, 0)$, ver (MS03). Por esse motivo, escrevemos simplesmente AGP em vez de $\operatorname{AGP}(\gamma)$.

O conjunto $\Omega\left(x^{k}, \gamma\right)$ pode ser considerado como uma aproximação linear do seguinte conjunto

$$
\left\{\begin{array}{ll}
h_{i}(z)=h_{i}\left(x^{k}\right), & \text { para todo } i \in\{1, \ldots, m\} \\
z \in \mathbb{R}^{n}: & g_{j}(z) \leq g_{j}\left(x^{k}\right), \quad \text { se } 0 \leq g_{j}\left(x^{k}\right) \\
g_{j}(z) \leq 0, & \text { se } \gamma<g_{j}\left(x^{k}\right)<0 \quad(\text { caso } \gamma \neq 0)
\end{array}\right\} .
$$

O conjunto 2.4 .12 é interpretado como o conjunto de pontos $z \in \mathbb{R}^{n}$ que são quase tão viáveis quanto o ponto atual $x^{k}$ e não deterioram a viabilidade obtida por $x^{k}$.

Para entender melhor a condição AGP, falaremos um pouco acerca dos métodos de restauração inexata. Os métodos de restauração inexata baseiam-se na ideia que em cada iteração, a viabilidade e a otimalidade são abordadas em diferentes fases, sem deteriorar muito o avanço obtido. Na fase de restauração, o método visa melhorar a viabilidade enquanto na fase de otimalidade o objetivo é melhorar a otimalidade, possivelmente deteriorando um pouco a viabilidade obtida. Usualmente, para algoritmos práticos de restauração inexata, a fase de viabilidade é realizada por algum procedimento relacionado com as características próprias do problema e a fase de otimalidade está relacionada com a direção de busca $d_{\gamma}\left(x^{k}\right):=P_{\Omega\left(x^{k}, \gamma\right)}\left(x^{k}-\nabla f\left(x^{k}\right)\right)-x^{k}$, que não deteriora muito a viabilidade obtida devido a que $x^{k}+d_{\gamma}\left(x^{k}\right)$ pertence a $\Omega\left(x^{k}, \gamma\right)$, aproximação linear de 2.4.12). Observemos que quando $d_{\gamma}\left(x^{k}\right)=0$, as condições KKT são satisfeitas em $x^{k}$.

Como mencionamos anteriormente, cada condição sequencial está associada com um critério natural de parada. Suponha que $\varepsilon_{\text {feas }}$ e $\varepsilon_{\text {opt }}$ são as tolerâncias referentes à viabilidade e à otimalidade, respectivamente, e seja $\left\{x^{k}\right\}$ uma sequência gerada por algum algoritmo de otimização. O critério natural de parada associado à condição AGP é declarar convergência em $x=x^{k}$ quando

$$
\left\|h\left(x^{k}\right)\right\| \leq \varepsilon_{\text {feas }}, \quad\left\|\max \left\{0, g\left(x^{k}\right)\right\}\right\| \leq \varepsilon_{\text {feas }} \text { e }\left\|P_{\Omega\left(x^{k}, \gamma\right)}\left(x^{k}-\nabla f\left(x^{k}\right)\right)-x^{k}\right\| \leq \varepsilon_{\text {opt }} .
$$

O Teorema 2.4 .3 mostra que a condição AGP é de fato uma genuína condição de otimalidade, (MS03, Teorema 2.1).

Teorema 2.4.3. Seja $x^{*} \in \Omega$ um minimizador local de 2.0.3) e $\gamma \in[-\infty, 0]$. Então a condição $A G P(\gamma)$ é satisfeita em $x^{*}$

Demonstração. Seja $x^{*}$ um minimizador local de 2.0.3), $\left\{\rho_{k}\right\}$ uma sequência de números positivos tal que $\rho_{k} \rightarrow \infty$ e $\gamma \in[-\infty, 0]$. Seguindo as mesmas linhas da demostração do Teorema 2.4.1, temos que existe uma sequência $\left\{x^{k}\right\} \operatorname{com} x^{k} \rightarrow x^{*}$ tal que

$$
\nabla f\left(x^{k}\right)+\sum_{i=1}^{m} \rho_{k} h_{i}\left(x^{k}\right) \nabla h_{i}\left(x^{k}\right)+\sum_{j: g_{j}\left(x^{k}\right) \geq 0} \rho_{k} \max \left\{0, g_{j}\left(x^{k}\right)\right\} \nabla g_{j}\left(x^{k}\right)=-\delta^{k},
$$

onde $\delta^{k}:=\left\|x^{k}-x^{*}\right\|\left(x^{k}-x^{*}\right) \rightarrow 0$. Adicionando e subtraindo $x^{k}$ adequadamente no lado esquerdo de 2.4.14, tomando a projeção sobre o conjunto $\Omega\left(x^{k}, \gamma\right)$ e da não expansividade da projeção segue 
que

$\left\|P_{\Omega\left(x^{k}, \gamma\right)}\left(x^{k}-\nabla f\left(x^{k}\right)\right)-P_{\Omega\left(x^{k}, \gamma\right)}\left(x^{k}+\sum_{i=1}^{m} \rho_{k} h_{i}\left(x^{k}\right) \nabla h_{i}\left(x^{k}\right)+\sum_{j: g_{j}\left(x^{k}\right) \geq 0} \rho_{k} g_{j}\left(x^{k}\right)+\nabla g_{j}\left(x^{k}\right)\right)\right\| \leq\left\|\delta^{k}\right\|$.

Como $\Omega\left(x^{k}, \gamma\right)$ é dado por meio de restrições lineares, o cone normal de $\Omega\left(x^{k}, \gamma\right)$ em $x=x^{k}$, $N_{\Omega\left(x^{k}, \gamma\right)}\left(x^{k}\right)$, é facilmente calculado. Usando o Teorema de Farkas temos

$$
\sum_{i=1}^{m} \rho_{k} h_{i}\left(x^{k}\right) \nabla h_{i}\left(x^{k}\right)+\sum_{j: g_{j}\left(x^{k}\right) \geq 0} \rho_{k} g_{j}\left(x^{k}\right) \nabla g_{j}\left(x^{k}\right) \in N_{\Omega\left(x^{k}, \gamma\right)}\left(x^{k}\right) .
$$

Da Proposição 2.1 .2 concluímos que

$$
P_{\Omega\left(x^{k}, \gamma\right)}\left(x^{k}+\sum_{i=1}^{m} \rho_{k} h_{i}\left(x^{k}\right) \nabla h_{i}\left(x^{k}\right)+\sum_{j: g_{j}\left(x^{k}\right) \geq 0} \rho_{k} g_{j}\left(x^{k}\right) \nabla g_{j}\left(x^{k}\right)\right)=x^{k} .
$$

Substituindo 2.4.17) em 2.4.15 temos $\left\|P_{\Omega\left(x^{k}, \gamma\right)}\left(x^{k}-\nabla f\left(x^{k}\right)\right)-x^{k}\right\| \leq\left\|\delta^{k}\right\|$, tomando limite na última expressão a condição $\operatorname{AGP}(\gamma)$ vale.

O seguinte teorema mostra que a condição AGP implica a condição AKKT.

Teorema 2.4.4. A condição AGP implica a condição AKKT.

Demonstração. Suponha que a condição AGP vale em $x^{*}$, então pela definição existe uma sequência $\left\{x^{k}\right\}$ tal que $y^{k}:=P_{\Omega\left(x^{k}, \gamma\right)}\left(x^{k}-\nabla f\left(x^{k}\right)\right)-x^{k} \rightarrow 0$ para algum $\gamma \in[-\infty, 0)$.

A projeção $P_{\Omega\left(x^{k}, \gamma\right)}\left(x^{k}-\nabla f\left(x^{k}\right)\right)=y^{k}+x^{k}$ é a única solução do problema:

$$
\text { Minimizar } \frac{1}{2}\left\|z-x^{k}+\nabla f\left(x^{k}\right)\right\|^{2} \quad \text { sujeito a } z \in \Omega\left(x^{k}, \gamma\right) \text {. }
$$

Desde que conjunto $\Omega\left(x^{k}, \gamma\right)$ é dado por restrições lineares, as condições KKT valem em $y^{k}+x^{k}$. Assim, existem multiplicadores $\lambda \in \mathbb{R}^{m}$ e $\mu \in \mathbb{R}_{+}^{p}$ tais que

$$
\nabla f\left(x^{k}\right)+y^{k}+\sum_{i=1}^{m} \lambda_{i}^{k} \nabla h_{i}\left(x^{k}\right)+\sum_{j=1}^{p} \mu_{j}^{k} \nabla g_{j}\left(x^{k}\right)=0
$$

e satisfazem as seguintes relações de complementaridade

$$
\begin{array}{rlrl}
\mu_{j}^{k}\left[g_{j}\left(x^{k}\right)+\left\langle\nabla g_{j}\left(x^{k}\right), y^{k}\right\rangle\right] & =0, & & \text { se } \gamma<g_{j}\left(x^{k}\right)<0 \\
\mu_{j}^{k}\left[\left\langle\nabla g_{j}\left(x^{k}\right), y^{k}\right\rangle\right] & =0, & \text { se } 0 \leq g_{j}\left(x^{k}\right) \\
\mu_{j}^{k} & =0, & & \text { caso contrário. }
\end{array}
$$

De 2.4.19 temos que $\lim _{k \rightarrow \infty} \nabla f\left(x^{k}\right)+\sum_{i=1}^{m} \lambda_{i}^{k} \nabla h_{i}\left(x^{k}\right)+\sum_{j=1}^{p} \mu_{j}^{k} \nabla g_{j}\left(x^{k}\right)=0$. Para provar que a condição AKKT vale, só falta demonstrar que $\mu_{j}^{k}=0$ para $j \notin J\left(x^{*}\right)$. Seja $j \notin J\left(x^{*}\right), \operatorname{logo}$ $g_{j}\left(x^{*}\right)<0$. Da continuidade temos que para $k$ suficientemente grande que $g_{j}\left(x^{k}\right)+\left\langle\nabla g_{j}\left(x^{k}\right), y^{k}\right\rangle<0$ e como consequência de $2.4 .20, \mu_{j}^{k}$ deve ser zero para todo $j \notin J\left(x^{*}\right)$.

Surpreendentemente, a condição sequencial AGP é estritamente mais forte que a condição AKKT, como mostra o próximo contra-exemplo.

Contra-exemplo 2.4.1. (A condição AKKT não implica AGP, (AHM11))

Em $\mathbb{R}^{2}$, consideremos o problema de otimização não linear

Minimizar $f\left(x_{1}, x_{2}\right)=-x_{2}$ sujeito a $h\left(x_{1}, x_{2}\right)=x_{1} x_{2}, \quad g\left(x_{1}, x_{2}\right)=-x_{1}$. 
Neste problema, a condição AGP não vale em $x^{*}=(0,1)^{\top}$ mas vale a condição AKKT.

A condição sequencial AGP é forte no sentido que implica "KKT ou não CPLD", ("(Hae09). A expressão "KKT ou não CPLD" pode ser considerada como uma medida da força da condição AGP. Na seção 3.1 .2 mostraremos a medida mais precisa da força da condição AGP como critério de parada para os algoritmos práticos.

No caso convexo temos o seguinte resultado, (MS03, Teorema 3.1), a qual basicamente diz junto a uma hipótese de viabilidade, a condição AGP é uma condição suficiente de otimalidade.

Teorema 2.4.5. (MS03, Teorema 3.1).

Suponha que as funções $f$ e $g_{j}, j=1, \ldots, p$ são convexas e que a função $h$ é uma função afim. Seja $\gamma \in[-\infty, 0]$. Suponha que $x^{*} \in \Omega$ e que $\left\{x^{k}\right\}$ é uma sequência $A G P(\gamma)$ para $x^{*}$ com a propriedade adicional $h\left(x^{k}\right)=0$ para todo $k \in \mathbb{N}$. Então $x^{*}$ é um minimo global de (2.0.3).

\subsubsection{Condição sequencial SAKKT}

Definição 2.4.3. Um ponto viável $x^{*} \in \Omega$ satisfaz a condição SAKKT, do inglês Strong AKKT para o problema 2.0.3 se existem sequências $\left\{x^{k}\right\} \subset \mathbb{R}^{n},\left\{\lambda^{k}\right\} \subset \mathbb{R}^{m},\left\{\mu^{k}\right\} \subset \mathbb{R}_{+}^{p}$ com as seguintes propriedades:

a) A sequência $\left\{x^{k}\right\}$ converge a $x^{*}$;

b) A sequência $\left\{\nabla f\left(x^{k}\right)+\sum_{i=1}^{m} \lambda_{i}^{k} \nabla h_{i}\left(x^{k}\right)+\sum_{j=1}^{p} \mu_{j}^{k} \nabla g_{j}\left(x^{k}\right)\right\}$ converge a zero;

c) Se $g_{j}\left(x^{k}\right)<0$ então temos que $\mu_{j}^{k}=0$.

A sequência $\left\{x^{k}\right\}$ é chamada de sequência SAKKT associada a $x^{*}$.

A condição SAKKT é uma genuína condição de otimalidade e implica a afirmação "KKT ou não CPLD", ainda mais, ela é equivalente à condição AGP(0).

Teorema 2.4.6. (Sch06, Teorema 1.2.6(b))

A condição SAKKT implica a condição $A G P(\gamma)$ para qualquer $\gamma \in[-\infty, 0]$.

Demonstração. Seja $x^{*} \in \Omega$ tal que a condição SAKKT vale em $x^{k}$ e seja $\gamma \in[-\infty, 0]$. Da definição de SAKKT temos que existem sequências $\left\{x^{k}\right\} \in \mathbb{R}^{n},\left\{\lambda^{k}\right\} \in \mathbb{R}^{m},\left\{\mu^{k}\right\} \in \mathbb{R}_{+}^{p}$ tais que $x^{k} \rightarrow x^{*}$ e

$$
\left(x^{k}-\nabla f\left(x^{k}\right)\right)-\left(x^{k}+\sum_{i=1}^{m} \lambda_{i}^{k} \nabla h_{i}\left(x^{k}\right)+\sum_{j: g_{j}\left(x^{k}\right) \geq 0} \mu_{j}^{k} \nabla g_{j}\left(x^{k}\right)\right) \rightarrow 0 .
$$

Usando a não-expansividade da projeção $P_{\Omega\left(x^{k}, \gamma\right)}$ temos que

$P_{\Omega\left(x^{k}, \gamma\right)}\left(x^{k}-\nabla f\left(x^{k}\right)\right)-P_{\Omega\left(x^{k}, \gamma\right)}\left(x^{k}+\sum_{i=1}^{m} \rho_{k} h_{i}\left(x^{k}\right) \nabla h_{i}\left(x^{k}\right)+\sum_{j: g_{j}\left(x^{k}\right) \geq 0} \rho_{k} \max \left\{0, g_{j}\left(x^{k}\right)\right\} \nabla g_{j}\left(x^{k}\right)\right) \rightarrow 0$.

Seguindo os mesmos argumentos da demonstração do Teorema 2.4.3, temos que a segunda projeção é igual a $x^{k}$ e como consequência a condição $\operatorname{AGP}(\gamma)$ vale.

Teorema 2.4.7. (Sch06, Teorema 1.2.6(c))

$A$ condição SAKKT é equivalente à condição $A G P(0)$.

Demonstração. Do Teorema anterior só falta provar que a condição $\operatorname{AGP}(0)$ implica a condição sequencial SAKKT. Da definição de $\operatorname{AGP}(0)$, existe uma sequência $\left\{x^{k}\right\}$ com $x^{k} \rightarrow 0$ tal que $y^{k}:=P_{\Omega\left(x^{k}, 0\right)}\left(x^{k}-\nabla f\left(x^{k}\right)\right)-x^{k} \rightarrow 0$.

Sabemos que $P_{\Omega\left(x^{k}, 0\right)}\left(x^{k}-\nabla f\left(x^{k}\right)\right)=y^{k}+x^{k}$ é a única solução do problema:

$$
\text { Minimizar } \frac{1}{2}\left\|z-x^{k}+\nabla f\left(x^{k}\right)\right\|^{2} \quad \text { sujeito a } z \in \Omega\left(x^{k}, 0\right) .
$$


Já que $\Omega\left(x^{k}, 0\right)$ é dada por restrições lineares, as condições KKT valem em $y^{k}+x^{k}$ e portanto existem multiplicadores $\lambda \in \mathbb{R}^{m}$ e $\mu \in \mathbb{R}_{+}^{p}$ tais que

$$
\nabla f\left(x^{k}\right)+y^{k}+\sum_{i=1}^{m} \lambda_{i}^{k} \nabla h_{i}\left(x^{k}\right)+\sum_{j=1}^{p} \mu_{j}^{k} \nabla g_{j}\left(x^{k}\right)=0
$$

e

$$
\mu_{j}^{k}\left[\left\langle\nabla g_{j}\left(x^{k}\right), y^{k}\right\rangle\right]=0, \text { se } 0 \leq g_{j}\left(x^{k}\right) \quad \text { e } \quad \mu_{j}^{k}=0, \text { caso contrário. }
$$

De 2.4.26 temos que $\lim _{k \rightarrow \infty} \nabla f\left(x^{k}\right)+\sum_{i=1}^{m} \lambda_{i}^{k} \nabla h_{i}\left(x^{k}\right)+\sum_{j=1}^{p} \mu_{j}^{k} \nabla g_{j}\left(x^{k}\right)=0$. Para provar que a condição SAKKT vale, só falta demonstrar que $\mu_{j}^{k}=0$ sempre que $g_{j}\left(x^{k}\right)<0$, mas isso é óbvio de (2.4.27).

Do Teorema anterior concluímos que a condição SAKKT é uma condição de otimalidade.

Observação 2.4.1. Apesar de SAKKT ser uma condição de otimalidade, ela não é uma condição de otimalidade desejada porque restringe muito o comportamento dos multiplicadores para certos métodos de otimização. Consideremos o seguinte exemplo (Sch06): Minimizar $x$ s.a $-x \leq 0$. Seja $x^{*}=0$ e suponha que existe um algoritmo que gera uma sequência $\left\{x^{k}\right\}$ que converge a $x^{*}$ e com a condição adicional que $x^{k}>0$ ( por exemplo, um método de pontos interiores). Essa sequência $\left\{x^{k}\right\}$ não pode ser uma sequência SAKKT, pois, no caso contrário, o item $\left.[c)\right]$ da definição de SAKKT, Definição 2.4.3, deve ser satisfeito, assim $\mu^{k}$ deve ser nulo e do item [b)] devemos ter que $\left\|\nabla f\left(x^{k}\right)+\sum_{i=1}^{n} \lambda_{i}^{k} \nabla h_{i}\left(x^{k}\right)+\sum_{j=1}^{p} \mu_{j}^{k} \nabla g_{j}\left(x^{k}\right)\right\|=\left\|\nabla f\left(x^{k}\right)\right\|=1$ converge para zero, que é obviamente impossivel.

\subsubsection{Condição sequencial L-AGP}

Quando o problema de otimização não-linear tem restrições lineares podemos considerar uma variação da condição sequencial AGP, a condição L-AGP, (AHM11). A ideia é separar as restrições lineares (tanto de igualdade e desigualdade) das restrições não lineares, e linearizar o conjunto das restrições não lineares. Fundamentaremos melhor essa ideia. Considere que, possivelmente depois de uma reordenação, o conjunto das restrições não lineares do problema (2.0.3), está dado por $\left\{h_{i}, g_{j}: i \in\left\{1, \ldots, m_{1}\right\}, j \in J_{1}\right\}$.

Denotemos por $\Omega_{L}$ o conjunto dos pontos que satisfazem todas as restrições lineares e defina

$$
\Omega_{N L}\left(x^{k},-\infty\right):=\left\{\begin{array}{ll}
\left\langle\nabla h_{i}\left(x^{k}\right), z-x^{k}\right\rangle=0, & \text { para } i \in\left\{1, \ldots, m_{1}\right\} \\
z \in \mathbb{R}^{n}: \begin{array}{ll}
\left\langle\nabla g_{j}\left(x^{k}\right), z-x^{k}\right\rangle \leq 0, \\
g_{j}\left(x^{k}\right)+\left\langle\nabla g_{j}\left(x^{k}\right), z-x^{k}\right\rangle \leq 0,
\end{array} & \text { se } \left.0 \leq g_{j}\left(x^{k}\right)<0, j \in x_{1}\right), j \in J_{1}
\end{array}\right\}
$$

Definição 2.4.4. Seja $x^{*}$ um ponto viável dizemos que se satisfaz a condição L-AGP para o problema (2.0.3), se existe uma sequência $\left\{x^{k}\right\} \subset \Omega_{L}$, com limite $x^{*}$, tal que

$$
P_{\Omega_{N L}\left(x^{k},-\infty\right) \cap \Omega_{L}}\left(x^{k}-\nabla f\left(x^{k}\right)\right)-x^{k} \rightarrow 0 .
$$

Teorema 2.4.8. (AHM11) Se $x^{*} \in \Omega$ é um minimizador local de (2.0.3) então L-AGP vale.

Teorema 2.4.9. AHM11) A condição L-AGP implica a condição AGP.

O Teorema 2.4 .9 apoia o ponto de vista de que, se um problema de otimização possui restrições lineares, é sensato preservar a viabilidade no que diz respeito a essas restrições, declarando convergência quando o critério AGP vale com certo nível de tolerância.

Do Teorema 2.4.9 observamos que a condição L-AGP implica "KKT ou não CPLD". Da mesma forma que "KKT ou não CPLD" fornece uma medida da força da condição AGP, ele também fornece uma medida da força da condição L-AGP. Na seção 3.1.5 mostraremos a medida mais precisa da força da condição L-AGP como critério de parada e provaremos que essa medida é mais forte que a medida associada à condição AGP. 


\subsubsection{Condição sequencial CAKKT}

Na busca de novas condições sequenciais, Andreani, Martínez e Svaiter em (AMS10) introduziram uma nova condição sequencial de otimalidade, a chamada CAKKT (Complementary approximate KKT). A motivação é, que mesmo em exemplos simples, como minimizar $\frac{1}{2}\left(x_{2}-2\right)^{2}$ sujeito a $x_{1}=0$ e $x_{1} x_{2}=0$, os critérios usuais não são suficientes para declarar convergência. Nesse exemplo em particular temos que a única solução é $(0,2)^{\top}$ mas qualquer ponto $x^{*}$ da forma $(\varepsilon, 1)^{\top}$ para $\varepsilon \geq 0$ pequeno, satisfaz a condição AKKT e, como consequência, cumpre qualquer critério de parada baseado na condição AKKT. Ainda mais, esses pontos cumprem as condições sequencias AGP e L-AGP, e portanto satisfazem qualquer critério prático baseado nessas condições. Assim, requisitos mais fortes são necessários para declarar convergência e como consequência, são obtidas condições sequenciais mais fortes.

Definição 2.4.5. Um ponto $x^{*}$ viável é um ponto CAKKT para o problema de programação (2.0.3), se existem sequências $\left\{x^{k}\right\} \subset \mathbb{R}^{n},\left\{\lambda^{k}\right\} \subset \mathbb{R}^{m}$ e $\left\{\mu^{k}\right\} \in \mathbb{R}_{+}^{p}$ com $\mu_{j}^{k}=0$ para todo $j \notin J\left(x^{*}\right)$ tais que $\left\{x^{k}\right\} \rightarrow x^{*}$,

$$
\lim _{k \rightarrow \infty}\left(\nabla f\left(x^{k}\right)+\sum_{i=1}^{m} \lambda_{i}^{k} \nabla h_{i}\left(x^{k}\right)+\sum_{j \in J\left(x^{*}\right)} \mu_{j}^{k} \nabla g_{j}\left(x^{k}\right)\right)=0
$$

$e$

$$
\lim _{k \rightarrow \infty}\left(\sum_{i=1}^{m}\left|\lambda_{i}^{k} h_{i}\left(x^{k}\right)\right|+\sum_{j \in J\left(x^{*}\right)}\left|\mu_{j}^{k} g_{j}\left(x^{k}\right)\right|\right)=0 .
$$

A condição CAKKT é mais forte do que outras versões aproximadas das condições KKT. Esse fato é essencialmente devido à presença da exigência (2.4.31) que está relacionada com a complementaridade. Como toda condição sequencial, CAKKT é uma condição de otimalidade.

Teorema 2.4.10. Seja $x^{*} \in \Omega$ um minimizador local de (2.0.3) então a condição CAKKT vale em $x^{*}$.

Demonstração. Seja $x^{*}$ um minimizador local de 2.0.3) e $\left\{\rho_{k}\right\}$ uma sequência de números positivos tal que $\rho_{k} \rightarrow \infty$.

Seguindo as mesma linhas da demostração do Teorema 2.4.1. temos que existe uma sequência $\left\{x^{k}\right\} \operatorname{com} x^{k} \rightarrow x^{*}$ tal que

$$
\nabla f\left(x^{k}\right)+\sum_{i=1}^{m} \rho_{k} h_{i}\left(x^{k}\right) \nabla h_{i}\left(x^{k}\right)+\sum_{j=1}^{p} \rho_{k} \max \left\{0, g_{j}\left(x^{k}\right)\right\} \nabla g_{j}\left(x^{k}\right)=-\left\|x^{k}-x^{*}\right\|\left(x^{k}-x^{*}\right) \rightarrow 0
$$

$\mathrm{e}$

$$
\sum_{i=1}^{m} \rho_{k} h_{i}\left(x^{k}\right)^{2}+\sum_{j=1}^{p} \rho_{k} \max \left\{0, g_{j}\left(x^{k}\right)\right\}^{2} \leq f\left(x^{*}\right)-f\left(x^{k}\right)-\frac{1}{2}\left\|x^{k}-x^{*}\right\|^{2} .
$$

Defina $\lambda_{i}^{k}:=\rho_{k} h_{i}\left(x^{k}\right)$ para $i \in\{1, \ldots, m\}$ e $\mu_{j}^{k}:=\rho_{k} \max \left\{g_{j}\left(x^{k}\right)\right\}$ para $j \in\{1, \ldots, p\}$. Da continuidade de $g$ temos que para $k$ grande $\mu_{j}^{k}=0$ para $j \notin J\left(x^{*}\right)$. De 2.4.32, 2.4.33 e de $\mu_{j}^{k}=0, j \in J\left(x^{*}\right)$ para $k$ suficientemente grande concluímos que 2.4.30 e 2.4.31 são satisfeitas, e logo CAKKT vale em $x^{*}$.

Associada a esta condição temos um critério natural de parada. Sejam $\varepsilon_{\text {feas }}$, $\varepsilon_{\text {opt }}$ e $\varepsilon_{\text {mult }}$ as tolerâncias referentes à viabilidade, à otimalidade e à condição 2.4.31), respectivamente. O critério natural de parada associada à condição CAKKT é declarar "convergência" quando 2.4.34 e 
2.4.35 são satisfeitas

$$
\left\|h\left(x^{k}\right)\right\| \leq \varepsilon_{\text {feas }}, \quad\left\|\max \left\{0, g\left(x^{k}\right)\right\}\right\| \leq \varepsilon_{\text {feas }}, \quad\left\|\nabla f\left(x^{k}\right)+\sum_{i=1}^{n} \lambda_{i}^{k} \nabla h_{i}\left(x^{k}\right)+\sum_{j=1}^{p} \mu_{j}^{k} \nabla g_{j}\left(x^{k}\right)\right\| \leq \varepsilon_{\text {opt }},
$$

e

$$
\left|\lambda_{i}^{k} h_{i}\left(x^{k}\right)\right| \leq \varepsilon_{\text {mult }}, \quad\left|\mu_{j}^{k} g_{j}\left(x^{k}\right)\right| \leq \varepsilon_{\text {mult }} \text { para todo } i \in\{1, \ldots, m\}, j \in\{1, \ldots, p\} .
$$

Claramente, a condição CAKKT implica a condição AKKT. De fato temos

Teorema 2.4.11. (AMS10, Teorema 4.1)

A condição CAKKT implica a condição $A G P(\gamma)$ para todo $\gamma \in[-\infty, 0]$. Além disso, se a sequência $\left\{x^{k}\right\}$ associada à definição de CAKKT cumpre com todas as restrições lineares (igualdades $e$ desigualdades), então a condição L-AGP vale.

Um fato interessante é que no caso convexo, a condição sequencial CAKKT é uma condição suficiente para a otimalidade (AMS10, Teorema 4.2) sem nenhuma hipótese adicional em contraposição às condições sequenciais AGP (MS03, Teorema 3.1) e SAKKT (HS11, Teorema 2.2).

Teorema 2.4.12. (AMS10, Teorema 4.2).

Suponha que as funções $f$ e $g_{j}, j=1, \ldots, p$ são convexas e que a função $h$ é uma função afim. Suponha que $x^{*} \in \Omega$ e a condição CAKKT vale em $x^{*}$. Então $x^{*}$ é um minimizador global de 2.0.3).

Mesmo sendo CAKKT uma condição forte, existem algoritmos que geram sequências cujo pontos limites satisfazem essa condição. Por exemplo, temos uma versão do método de lagrangiano aumentado sob a hipótese adicional que a função $\Phi(x)=\|h(x)\|^{2}+\|\max \{0, g(x)\}\|^{2}$ cumpra a desigualdade generalizada de Lojasiewicz, ver (AMS10, Teorema 5.1).

Do Teorema 2.4.11 temos que a condição CAKKT implica a condição SAKKT, mas pela Observação 2.4.1, sabemos que SAKKT não é uma condição de otimalidade desejável, então por quê estaríamos interessados na condição CAKKT a qual é ainda mais restritiva que SAKKT? Primeiro porque, como mencionamos anteriormente, apesar de ser CAKKT uma condição de otimalidade mais forte que SAKKT, existem algoritmos práticos que geram sequências CAKKT (AMS10) e segundo, o exemplo da Observação 2.4.1 não se aplica à condição CAKKT, porque os multiplicadores que aparecem na definição de CAKKT não são necessariamente os mesmos multiplicadores que aparecem em SAKKT.

A Figura 2.5 mostra as implicações existentes entre as condições sequenciais.

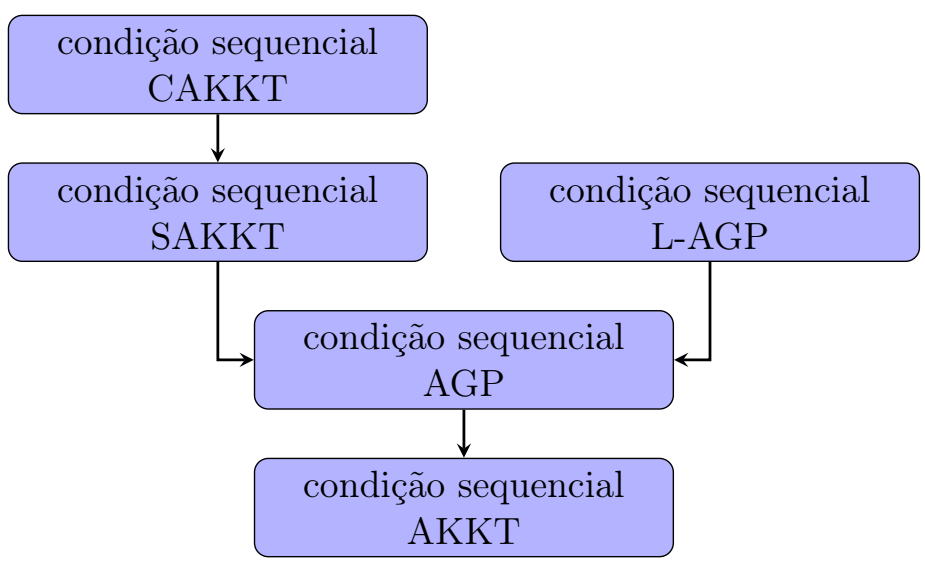

Figura 2.5: Relações entre as condições sequenciais de otimalidade de primeira ordem. 


\section{Capítulo 3}

\section{Condições de Qualificação associadas com as Condições Sequenciais de Otimalidade de primeira ordem}

Dizemos que uma condição de qualificação tem claras implicações algorítmicas se é possível provar a convergência de métodos numéricos sob essa condição. Neste capítulo, nosso principal interesse é o estudo de condições de qualificação com claras implicações algorítmicas, de preferência fracas, já que condições de qualificação fracas implicam condições de otimalidade fortes da forma (2.0.7). Um exemplo de uma condição de qualificação com claras implicações algorítmicas temos a condição CPLD introduzida por Qi e Wei em (QW00). Os autores apresentaram um método particular de programação quadrática sequencial com convergência a pontos limites que satisfazem a condição "KKT ou não CPLD". Então, sob a condição CPLD, esse método de programação quadrática sequencial tem convergência a pontos KKT. O fato de CPLD ser uma condição de qualificação foi provado posteriormente por Andreani, Martínez e Schuverdt (AMS05), que ainda mostraram as relações existentes entre essa e as outras condições de qualificação conhecidas na época. Em artigos posteriores, Andreani, Birgin, Martínez e Schuverdt (ABMS07, ABMS08) provaram que a condição de qualificação CPLD pode ser usada para a análise de convergência do método de lagrangiano aumentado, um avanço significativo pois as provas anteriores eram baseadas na condição de regularidade (LICQ), claramente mais restritiva. A procura por condições de qualificação fracas com claras implicações algorítmicas motivou uma serie de artigos (AHSS12b, AHSS12a) dando origem a condições de qualificação como RCPLD, CRSC ou CPG.

Para entender a relação entre condições de qualificação e algoritmos, começaremos analisando a mais popular das condições sequencias de otimalidade, a condição AKKT. Suponha que P seja uma condição que depende somente das restrições do problema e é tal que

$$
\mathrm{AKKT}+\mathrm{P} \Longrightarrow \mathrm{KKT}
$$

Como a condição AKKT é uma condição de otimalidade, a condição P é uma condição de qualificação. Ainda mais, se temos algum algoritmo prático que gera sequências cujos pontos limites satisfazem a condição AKKT temos, sob a condição $\mathrm{P}$, que o algoritmo gera ponto limites onde as condições KKT valem. O interessante é que, de fato, existem algoritmos práticos que naturalmente geram pontos AKKT, como no caso do lagrangiano aumentado (BM14) ou o método de programação quadrática sequencial de (QW00). Assim, a condição P é uma condição de qualificação com claras implicações algorítmicas. No caso especifico da condição AKKT, a condição P pode ser, por exemplo, a condição de qualificação MFCQ, RCPLD, CRCS ou mesma a condição CPG mas não a condição de Abadie nem a Pseudonormalidade (seção 3.3).

Uma pergunta natural é a seguinte: dado que a condição AKKT é uma genuína condição de otimalidade, obviamente associada com o critério de parada de algoritmos práticos, por que devemos nos preocupar com condições de qualificações nas quais AKKT implica KKT (condição que não é 
uma condição de otimalidade)? O motivo é que, para famílias de problemas importantes, a condição AKKT não é suficientemente forte para fornecer uma confiável deteç̧ão da otimalidade. O exemplo clássico é uma versão simplificada do problema MPCC (mathematical program with complementarity constraints). Consideremos o problema de minimizar $x_{2}$ sujeito a $x_{1} \geq 0$ e $x_{1} x_{2}=0$. O ponto viável $x^{*}=(0,1)^{\top}$ é um ponto AKKT (basta considerar a sequência $x_{1}^{k}=1 / k, x_{2}^{k}=1$ e multiplicadores $\lambda^{k}=\mu^{k}=k$ ) mas o ponto $x^{*}$ não tem nenhuma relação com o problema a minimizar, ele não é nem minimizador nem um ponto KKT. Mas seria satisfeita por qualquer critério de parada baseado na condição AKKT. Certamente, isso não acontece, se no ponto $x^{*} \in \Omega$ valesse qualquer condição $\mathrm{P}$ que satisfaz a expressão (3.0.1).

Nossa contribuição nesse capítulo é mostrar a condição P mínima, não somente para o caso da condição sequencial AKKT, mas também para cada uma das outras condições sequenciais de otimalidade AGP, SAKKT, L-AGP e CAKKT (seção 3.1), mostrando as implicações existentes entre elas (seção 3.2) e as relações com as outras condições de qualificação conhecidas na literatura, ver (AMRS15). Em particular provaremos que essas condições são estritamente mais fortes que a condição de Abadie e não têm relação nenhuma com a Quasinormalidade ou com a Pseudonormalidade (seção 3.3). As novas condições de qualificação desempenham para a respectiva condição sequencial o mesmo papel que a condição de Guignard desempenha para a otimalidade.

\subsection{Novas condiçoes de qualificação}

\subsubsection{CQ associada a AKKT: Propriedade de Continuidade do Cone}

Seja $x^{*} \in \Omega$ um ponto viável, defina

$$
K(x):=\left\{\sum_{i=1}^{m} \lambda_{i} \nabla h_{i}(x)+\sum_{j \in J\left(x^{*}\right)} \mu_{j} \nabla g_{j}(x): \mu_{j} \in \mathbb{R}_{+}, \lambda_{i} \in \mathbb{R}\right\} .
$$

O conjunto $K(x)$ é um cone fechado convexo que coincide com o cone polar linearizado $L_{\Omega}^{\circ}\left(x^{*}\right)$ em $x^{*} \in \Omega$. Da expressão (3.1.1) temos que o cone $K(x)$ pode ser considerado como uma pertubação do cone polar linearizado ao redor do $x^{*} \in \Omega$. Já que $K\left(x^{*}\right)$ coincide com $L_{\Omega}^{\circ}\left(x^{*}\right)$, as condições KKT podem ser escritas como $-\nabla f\left(x^{*}\right) \in K\left(x^{*}\right)$. Ainda mais, usando o cone $K(x)$ podemos re-escrever a condição CPG de uma forma mais geométrica. Lembremos que, dado os subconjuntos de índices $I^{\prime} \subset I, J^{\prime} \subset J_{-}$e $J_{+}=J\left(x^{*}\right) \backslash J_{-}$, a notação $K_{I^{\prime}, J^{\prime}}(x)$ representa o seguinte cone

$$
K_{I^{\prime}, J^{\prime}}(x):=\left\{\sum_{i \in I^{\prime}} \lambda_{i} \nabla h_{i}(x)+\sum_{\ell \in J^{\prime}} \gamma_{\ell} \nabla g_{\ell}(x)+\sum_{j \in J_{+}} \mu_{j} \nabla g_{j}(x): \mu_{j} \in \mathbb{R}_{+}, \lambda_{i}, \gamma_{r} \in \mathbb{R}\right\} .
$$

Assim, a condição CPG vale em $x^{*}$ se existem $I^{\prime} \subset I, J^{\prime} \subset J_{-}$e uma vizinhança $V$ de $x^{*}$ tal que:

1. Os gradientes $\left\{\nabla h_{i}\left(x^{*}\right), \nabla g_{j}\left(x^{*}\right): i \in I^{\prime}, j \in J^{\prime}\right\}$ são linearmente independentes;

2. Para todo $x \in V$

$$
K(x) \subset K_{I^{\prime}, J^{\prime}}(x) .
$$

Claramente, ambos cones coincidem em $x^{*}$, isto é, $K\left(x^{*}\right)=K_{I^{\prime}, J^{\prime}}\left(x^{*}\right)$.

Definição 3.1.1. Seja $x^{*}$ um ponto viável. Dizemos que a Propriedade de Continuidade do Cone $(C C P)$ vale em $x^{*} \in \Omega$ se a multifunção $\mathbb{R}^{n} \ni x \rightrightarrows K(x)$ é semicontínua exteriormente em $x^{*}$, isto é:

$$
\limsup _{x \rightarrow x^{*}} K(x) \subset K\left(x^{*}\right) .
$$


Note que devido à continuidade dos gradientes e da própria definição de $K(x)$, a multifunção $\mathbb{R}^{n} \ni x \rightrightarrows K(x)$ é sempre semicontínua interiormente. Por essa razão, a semi-continuidade exterior é suficiente para a continuidade de $K(x)$ em $x^{*}$.

A condição sequencial AKKT está naturalmente associada com a Propriedade da Continuidade do Cone. Da definição de AKKT, Definição 2.4.1, existem sequências $\left\{x^{k}\right\} \subset \mathbb{R}^{n},\left\{\lambda^{k}\right\} \subset \mathbb{R}^{m} \mathrm{e}$ $\left\{\mu^{k}\right\} \subset \mathbb{R}_{+}^{p}$ com $\mu_{j}^{k}=0$ para $j \notin J\left(x^{*}\right)$, tais que $\lim _{k \rightarrow \infty} x^{k}=x^{*} \mathrm{e}$

$$
\lim _{k \rightarrow \infty}\left(\nabla f\left(x^{k}\right)+\sum_{i=1}^{m} \lambda_{i}^{k} \nabla h_{i}\left(x^{k}\right)+\sum_{j \in J\left(x^{*}\right)} \mu_{j}^{k} \nabla g_{j}\left(x^{k}\right)\right)=0 .
$$

A expressão (3.1.4) diz que se a condição AKKT é satisfeita os gradientes $-\nabla f\left(x^{k}\right)$ se aproximam do cone $K\left(x^{k}\right)$ quando $k$ vai para o infinito.

O seguinte teorema mostra que a condição CCP desempenha, com respeito à condição AKKT, o mesmo papel que a condição de Guignard desempenha com respeito à otimalidade local. A condição de Guignard é a condição de qualificação mínima para garantir que todo minimizador local cumple as condições KKT. Do mesmo modo que CCP é a condição mínima com respeito às restrições que garante que a condição AKKT implica às condições KKT.

Teorema 3.1.1. A condição CCP é a propriedade minima com respeito às restrições segundo a qual a condição AKKT implica a validade das condições KKT, independentemente da função objetivo.

Demonstração. Primeiro mostraremos que se a condição CCP vale, então a condição sequencial AKKT implica as condições KKT independentemente da função objetivo. Seja $f$ uma função objetivo tal que a condição sequencial AKKT vale em $x^{*}$. Então, pela definição, existem sequências $\left\{x^{k}\right\} \rightarrow x^{*},\left\{\lambda^{k}\right\} \subset \mathbb{R}^{m},\left\{\mu^{k}\right\} \subset \mathbb{R}_{+}^{p}$ com $\mu_{j}^{k}=0$ para $j \notin J\left(x^{*}\right)$ e $\left\{\zeta^{k}\right\} \subset \mathbb{R}^{n}$ tais que

$$
\zeta^{k}:=\nabla f\left(x^{k}\right)+\sum_{i=1}^{m} \lambda_{i}^{k} \nabla h_{i}\left(x^{k}\right)+\sum_{j \in J\left(x^{*}\right)} \mu_{j}^{k} \nabla g_{j}\left(x^{k}\right) \rightarrow 0 .
$$

Defina $\omega^{k}:=\sum_{i=1}^{m} \lambda_{i}^{k} \nabla h_{i}\left(x^{k}\right)+\sum_{j \in J\left(x^{*}\right)} \mu_{j}^{k} \nabla g_{j}\left(x^{k}\right)$. De 3.1.5 e da definição do cone $K(x)$ temos que

$$
\omega^{k} \in K\left(x^{k}\right) \text { e } \omega^{k}=\zeta^{k}-\nabla f\left(x^{k}\right)
$$

Tomando limites em 3.1.6), usando a continuidade do gradiente de $f$ e que $\zeta^{k} \rightarrow 0$, obtemos que

$$
-\nabla f\left(x^{*}\right)=\lim _{k \rightarrow \infty} \omega^{k} \in \limsup _{x \rightarrow x^{*}} K(x) \subset K\left(x^{*}\right),
$$

onde a última inclusão segue da propriedade da continuidade do cone. Desta forma, $-\nabla f\left(x^{*}\right) \in$ $K\left(x^{*}\right)$, que é equivalente a dizer que as condições KKT valem em $x^{*}$.

A seguir, provaremos que, se a condição sequencial AKKT implica as condições KKT para toda função objetivo, então a condição CCP vale.

Seja $\omega^{*} \in \lim \sup _{x \rightarrow x^{*}} K(x)$, logo existem sequências $\left\{x^{k}\right\}$ e $\left\{\omega^{k}\right\}$ tais que $x^{k} \rightarrow x^{*}, \omega^{k} \rightarrow \omega^{*}$ e $\omega^{k} \in K\left(x^{k}\right)$. Defina $f(x):=-\left\langle w^{*}, x\right\rangle$ para todo $x \in \mathbb{R}^{n}$. Note que a condição AKKT vale em $x^{*}$ para esta função com $\left\{x^{k}\right\}$ como a sequência AKKT associada porque $\nabla f\left(x^{k}\right)+\omega^{k}=-\omega^{*}+\omega^{k} \rightarrow 0$. Logo, por hipótese, as condições KKT valem em $x^{*}$, isto é, $-\nabla f\left(x^{*}\right)=\omega^{*} \in K\left(x^{*}\right)$.

Já que a condição sequencial AKKT é uma condição de otimalidade, temos como corolário.

Corolário 3.1.2. A condição CCP é uma condição de qualificação.

A convergência para pontos KKT de vários algoritmos práticos que geram sequências AKKT (como por exemplo, o método de programação quadrática sequencial de Qi e Wei (QW00), o método de pontos interiores de Chen e Goldfarb (CG06) e os métodos de lagrangiano aumentado de (ABMS08, BM14), entre outros) tem sido provada assumindo diferentes condições de qualificação. 
Com o Teorema 3.1.1 à disposição, em todos os casos, podemos substituir a condição de qualificação usada pela condição CCP, que é mais fraca, e esse tipo de resultado não pode ser melhorado usando outras condições de qualificação. Para mais informações e detalhes ver (AMRS16).

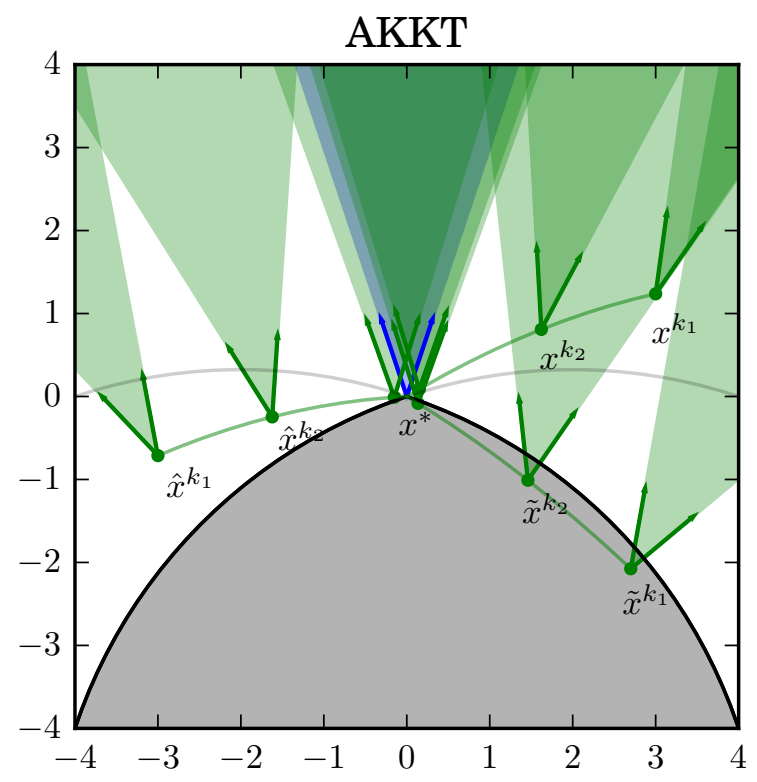

Figura 3.1: Exemplo do cone associado à condição AKKT.

Na Figura 3.1 a área sombreada é o conjunto viável composto pela interseção de dois círculos. O ponto de interesse é $x^{*}=0$. Temos três tipos de sequências com limite $x^{*}$. As sequências $\left\{x^{k}\right\}$, $\left\{\hat{x}^{k}\right\}$ e $\left\{\tilde{x}^{k}\right\}$ são, respectivamente, inviável com respeito a ambas restrições, com respeito a uma restrição e viável. A condição CCP basicamente diz que os possíveis limites dos vetores do cone verde devem pertencer ao cone azul que é o normal do cone tangente linearizado em $x^{*}$. Note que o cone associado a AKKT, sempre tem em consideração todas as restrições ativas do ponto $x^{*}$.

\subsubsection{CQ associada a AGP}

Definição 3.1.2. Seja $x^{*} \in \Omega$ dizemos que $x^{*}$ satisfaz a condição do AGP regular se a multifunção

$$
\mathbb{R}^{n} \times \mathbb{R}^{n} \ni(x, \varepsilon) \rightrightarrows N_{\Omega(x,-\infty)}(x+\varepsilon)
$$

é semicontínua exteriormente em $\left(x^{*}, 0\right)$. Isto é:

$$
\limsup _{(x, \varepsilon) \rightarrow\left(x^{*}, 0\right)} N_{\Omega(x,-\infty)}(x+\varepsilon) \subset N_{\Omega\left(x^{*},-\infty\right)}\left(x^{*}\right)=K\left(x^{*}\right) .
$$

Como consequência do Teorema de Farkas, o cone normal $N_{\Omega(x,-\infty)}(x+\varepsilon)$ pode ser calculado explicitamente:

Proposição 3.1.3. Todo elemento do cone $N_{\Omega(x,-\infty)}(x+\varepsilon)$ é da forma

$$
\sum_{i=1}^{m} \lambda_{i} \nabla h_{i}(x)+\sum_{j: g_{j}(x) \geq 0} \mu_{1 j} \nabla g_{j}(x)+\sum_{j: g_{j}(x)<0} \mu_{2 j} \nabla g_{j}(x),
$$

onde $\lambda_{i} \in \mathbb{R}, \mu_{1 j} \in \mathbb{R}_{+}, \mu_{2 j} \in \mathbb{R}_{+}$e se satisfazem as relações

$$
\mu_{1 j}\left(\left\langle\nabla g_{j}(x), \varepsilon\right\rangle\right)=0, \text { se } g_{j}(x) \geq 0 \quad \text { e } \mu_{2 j}\left(g_{j}(x)+\left\langle\nabla g_{j}(x), \varepsilon\right\rangle\right)=0 \text {, se } g_{j}(x)<0 \text {. }
$$

O Teorema 3.1.4 mostra que a semicontinuidade exterior de $N_{\Omega(x,-\infty)}(x+\varepsilon)$ em $\left(x^{*}, 0\right)$ é a condição mínima para garantir que a condição sequencial AGP implica as condições KKT independente 
da função objetivo. Assim, a condição AGP regular desempenha, com respeito à condição AGP, o mesmo papel que a condição de Guignard desempenha com respeito à otimalidade local.

Teorema 3.1.4. A condição AGP regular é a minima condição com respeito somente às restrições para a qual a condição AGP implica a condição KKT, independentemente da função objetivo.

Demonstração. Começaremos por demonstrar que, sob a condição AGP regular, AGP implica KKT. Seja $\gamma \in[-\infty, 0)$ e seja $f$ uma função objetivo para a qual a condição $\operatorname{AGP}(\gamma)$ vale. Pela definição de $\operatorname{AGP}(\gamma)$ existe uma sequência $\left\{x^{k}\right\} \subset \mathbb{R}^{n}$, tal que $x^{k} \rightarrow x^{*}$ e $P_{\Omega\left(x^{k}, \gamma\right)}\left(x^{k}-\nabla f\left(x^{k}\right)\right)-x^{k} \rightarrow 0$. Defina $y^{k}:=P_{\Omega\left(x^{k}, \gamma\right)}\left(x^{k}-\nabla f\left(x^{k}\right)\right)$ e $\varepsilon^{k}:=y^{k}-x^{k}$. Note que, da condição AGP, $\lim _{k \rightarrow \infty} \varepsilon^{k}=0$. Da Proposição 2.1.2

$$
\omega^{k}:=x^{k}-\nabla f\left(x^{k}\right)-y^{k} \in N_{\Omega\left(x^{k}, \gamma\right)}\left(y^{k}=x^{k}+\varepsilon^{k}\right) .
$$

Como o cone normal $N_{\Omega\left(x^{k}, \gamma\right)}\left(y^{k}\right) \subset N_{\Omega\left(x^{k},-\infty\right)}\left(y^{k}\right)$ (porque $\left.\Omega(x,-\infty) \subset \Omega(x, \gamma)\right)$ temos que a sequência $\left\{\omega^{k}\right\}$ satisfaz às seguintes relações:

$$
\omega^{k} \in N_{\Omega\left(x^{k},-\infty\right)}\left(x^{k}+\varepsilon^{k}\right) \text { e } \omega^{k}=x^{k}-\nabla f\left(x^{k}\right)-y^{k}=-\nabla f\left(x^{k}\right)-\varepsilon^{k} .
$$

Tomando limite na expressão anterior e usando a continuidade do gradiente de $f$

$$
-\nabla f\left(x^{*}\right)=\lim _{k \rightarrow \infty} \omega^{k} \in \limsup _{(x, \varepsilon) \rightarrow\left(x^{*}, 0\right)} N_{\Omega(x,-\infty)}(x+\varepsilon) \subset N_{\Omega\left(x^{*},-\infty\right)}\left(x^{*}\right) .
$$

Assim, $-\nabla f\left(x^{*}\right)$ pertence a $N_{\Omega\left(x^{*},-\infty\right)}\left(x^{*}\right)=K\left(x^{*}\right)$ e as condições KKT valem em $x^{*}$.

Agora, provaremos que se a condição sequencial AGP implica as condições KKT para toda função objetivo, então a condição AGP regular vale.

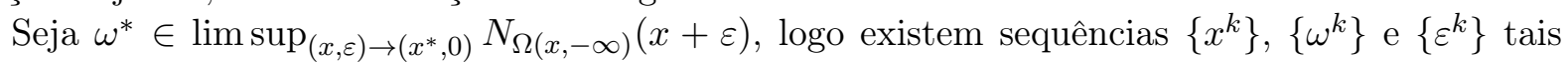
que $x^{k} \rightarrow x^{*}, \varepsilon^{k} \rightarrow 0, \omega^{k} \rightarrow \omega^{*}$ e $\omega^{k} \in N_{\Omega\left(x^{k},-\infty\right)}\left(x^{k}+\varepsilon^{k}\right)$. Defina a função $f(x):=-\left\langle w^{*}, x\right\rangle$ para todo $x \in \mathbb{R}^{n}$. Mostraremos que a condição sequencial $\operatorname{AGP}(-\infty)$ vale em $x^{*}$ para essa escolha de $f$. Nosso objetivo é provar que $\lim _{k \rightarrow \infty} P_{\Omega\left(x^{k},-\infty\right)}\left(x^{k}-\nabla f\left(x^{k}\right)\right)-x^{k}=0$. Defina $y^{k}:=x^{k}+\varepsilon^{k} \mathrm{e}$ $z^{k}:=P_{\Omega\left(x^{k},-\infty\right)}\left(x^{k}-\nabla f\left(x^{k}\right)\right)=P_{\Omega\left(x^{k},-\infty\right)}\left(x^{k}+\omega^{*}\right)$. Note que, como $\omega^{k} \in N_{\Omega\left(x^{k},-\infty\right)}\left(y^{k}\right)$, temos, pela Proposição 2.1.2, que $P_{\Omega\left(x^{k},-\infty\right)}\left(\omega^{k}+y^{k}\right)=y^{k}$. Usando a desigualdade triangular e a não expansividade da projeção ortogonal, segue que

$$
\left\|z^{k}-y^{k}\right\|=\left\|P_{\Omega\left(x^{k},-\infty\right)}\left(x^{k}+\omega^{*}\right)-P_{\Omega\left(x^{k},-\infty\right)}\left(\omega^{k}+y^{k}\right)\right\| \leq\left\|\omega^{*}-\omega^{k}\right\|+\left\|y^{k}-x^{k}\right\| .
$$

Tomando limite em 3.1.13 e usando $\lim _{k \rightarrow \infty}\left(y^{k}-x^{k}\right)=\lim _{k \rightarrow \infty} \varepsilon^{k}=0$ temos $\lim _{k \rightarrow \infty} z^{k}-y^{k}=0$. Como consequência

$$
\lim _{k \rightarrow \infty} P_{\Omega\left(x^{k},-\infty\right)}\left(x^{k}-\nabla f\left(x^{k}\right)\right)-x^{k}=\lim _{k \rightarrow \infty} z^{k}-x^{k}=\lim _{k \rightarrow \infty}\left(z^{k}-y^{k}\right)+\lim _{k \rightarrow \infty}\left(y^{k}-x^{k}\right)=0 .
$$

Portanto, a condição sequencial AGP vale em $x^{*}$ e da hipótese concluímos que as condições KKT valem em $x^{*}$, isto é, $-\nabla f\left(x^{*}\right)=\omega^{*}$ pertence a $N_{\Omega\left(x^{*},-\infty\right)}\left(x^{*}\right)=K\left(x^{*}\right)$.

Uma vez que a condição sequencial AGP é uma condição de otimalidade temos

Corolário 3.1.5. A condição AGP regular é uma condição de qualificação.

Na Figura 3.2. mostramos um exemplo do cone associado à condição AGP. O conjunto viável é formada pela interseção de dois círculos e o ponto de interesse é $x^{*}=0$. Analogamente à Figura 3.1 temos três tipos de sequências que convergem a $x^{*},\left\{x^{k}\right\},\left\{\hat{x}^{k}\right\}$ e $\left\{\tilde{x}^{k}\right\}$. A condição AGP-regular basicamente diz que todos os possíveis pontos limites de vetores do cone verde deve pertencer ao cone azul, que é $L_{\Omega}^{\circ}\left(x^{*}\right)$. Os cones associados à condição AGP só tomam em consideração as restrições que são ativas no mesmo ponto. É interessante notar o efeito das possíveis perturbações, que vem dado por $\left\{\varepsilon^{k}\right\}$. Seus possíveis valores na Figura 3.2 estão representadas por círculos sombreados. 


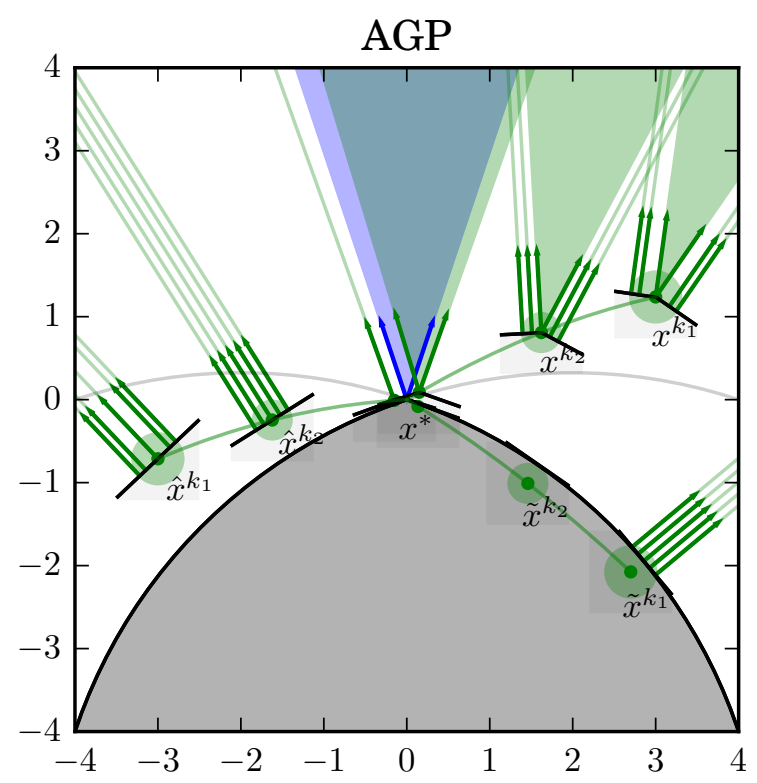

Figura 3.2: Exemplo do cone associado à condição AGP.

Eles permitem ter em consideração os gradientes das restrições que serão ativas em $x^{*}$ mas não para uma sequência estritamente viável. Observe o ponto $\tilde{x}^{k_{1}}$ na Figura 3.2 .

\subsubsection{CQ associada a CAKKT}

Seja $x^{*}$ um ponto viável. Dados $x \in \mathbb{R}^{m}$ e $r \in \mathbb{R}_{+}$. Defina

$K_{C}(x, r):=\left\{\sum_{i=1}^{m} \lambda_{i} \nabla h_{i}(x)+\sum_{j \in J\left(x^{*}\right)} \mu_{j} \nabla g_{j}(x): \sum_{i=1}^{m}\left|\lambda_{i} h_{i}(x)\right|+\sum_{j \in J\left(x^{*}\right)}\left|\mu_{j} g_{j}(x)\right| \leq r, \lambda_{i} \in \mathbb{R}, \mu_{j} \geq 0\right\}$.

O conjunto $K_{C}(x, r)$ é um conjunto convexo mas não um cone, além disso para todo $\alpha>0$ vale $\alpha K_{C}(x, r)=K_{C}(x, \alpha r)$ e $K_{C}(x, \infty)=K(x)$ para todo $x \in \mathbb{R}^{n}$. A seguir apresentamos a nova condição de qualificação associada à condição sequencial CAKKT.

Definição 3.1.3. Seja $x^{*}$ um ponto viável. Dizemos que $x^{*}$ satisfaz a condição CAKKT regular se a multifunção

$$
\mathbb{R}^{n} \times \mathbb{R}_{+} \ni(x, r) \rightrightarrows K_{C}(x, r)
$$

é semicontínua exteriormente em $\left(x^{*}, 0\right)$, em outras palavras,

$$
\limsup _{(x, r) \rightarrow\left(x^{*}, 0\right)} K_{C}(x, r) \subset K_{C}\left(x^{*}, 0\right)=K\left(x^{*}\right) .
$$

Teorema 3.1.6. Seja $x^{*} \in \Omega$. Então, $x^{*}$ é um ponto CAKKT regular se, e somente se a condição sequêncial CAKKT em $x^{*}$ implica a condição KKT em $x^{*}$, independentemente da função objetivo.

Demonstração. Começaremos por demonstrar que se $x^{*}$ é um ponto CAKKT regular então a condição CAKKT implica as condições KKT para toda função objetivo.

Seja $f$ qualquer função objetivo tal que a condição sequencial CAKKT é válida em $x^{*}$. Assim, existem sequências $\left\{x^{k}\right\} \subset \mathbb{R}^{n},\left\{\lambda^{k}\right\} \subset \mathbb{R}^{m},\left\{\mu^{k}\right\} \subset \mathbb{R}_{+}^{p}$ com $\mu_{j}^{k}=0$ para $j \notin J\left(x^{*}\right),\left\{\zeta^{k}\right\} \subset \mathbb{R}^{m} \mathrm{e}$ $\left\{r^{k}\right\} \subset \mathbb{R}_{+}$tais que $\lim _{k \rightarrow \infty} x^{k}=x^{*}, \zeta^{k}:=\nabla f\left(x^{k}\right)+\sum_{i=1}^{m} \lambda_{i}^{k} \nabla h_{i}\left(x^{k}\right)+\sum_{j \in J\left(x^{*}\right)} \mu_{j}^{k} \nabla g_{j}\left(x^{k}\right) \rightarrow 0$ e $r^{k}:=\sum_{i=1}^{m}\left|\lambda_{i}^{k} h_{i}\left(x^{k}\right)\right|+\sum_{j \in J\left(x^{*}\right)}\left|\mu_{j}^{k} g_{j}\left(x^{k}\right)\right| \rightarrow 0$. 
Defina $\omega^{k}:=\sum_{i=1}^{m} \lambda_{i}^{k} \nabla h_{i}\left(x^{k}\right)+\sum_{j \in J\left(x^{*}\right)} \mu_{j}^{k} \nabla g_{j}\left(x^{k}\right)$. A sequência $\left\{\omega^{k}\right\}$ satisfaz

$$
\omega^{k} \in K_{C}\left(x^{k}, r^{k}\right) \text { e } \omega^{k}=\zeta^{k}-\nabla f\left(x^{k}\right) .
$$

De $\zeta^{k} \rightarrow 0$ e $\nabla f\left(x^{k}\right) \rightarrow \nabla f\left(x^{*}\right)$ temos que a sequência $\left\{\omega^{k}\right\}$ converge a $-\nabla f\left(x^{*}\right)$ e da definição de limite exterior concluímos que

$$
-\nabla f\left(x^{*}\right)=\lim _{k \rightarrow \infty} \omega^{k} \in \limsup _{(x, r) \rightarrow\left(x^{*}, 0\right)} K_{C}(x, r) \subset K_{C}\left(x^{*}, 0\right)=K\left(x^{*}\right) .
$$

Assim, as condições KKT valem.

A seguir, mostraremos que se CAKKT implica KKT para toda função objetivo então $x^{*}$ é um ponto CAKKT regular. Assim, devemos provar a inclusão $\lim \sup _{(x, r) \rightarrow\left(x^{*}, 0\right)} K_{C}(x, r) \subset K\left(x^{*}\right)$. Considere $\omega^{*} \in \lim \sup _{(x, r) \rightarrow\left(x^{*}, 0\right)} K_{C}(x, r)$, logo existem sequências $\left\{x^{k}, \omega^{k}, r^{k}\right\}$ tais que $x^{k} \rightarrow x^{*}$, $\omega^{k} \rightarrow \omega^{*}, r^{k} \rightarrow 0$ e $\omega^{k} \in K_{C}\left(x^{k}, r^{k}\right)$. Defina $f(x):=-\left\langle w^{*}, x\right\rangle$ para todo $x \in \mathbb{R}^{n}$. Vejamos que CAKKT vale em $x^{*}$ para a função objetivo $f$.

Como $\omega^{k} \in K_{C}\left(x^{k}, r^{k}\right)$ existem multiplicadores $\left\{\lambda^{k}\right\} \subset \mathbb{R}^{m},\left\{\mu^{k}\right\} \subset \mathbb{R}_{+}^{p}$ com $\mu_{j}^{k}=0$ para $j \notin J\left(x^{*}\right)$ tais que

$$
\omega^{k}=\sum_{i=1}^{m} \lambda_{i}^{k} \nabla h_{i}\left(x^{k}\right)+\sum_{j \in J\left(x^{*}\right)} \mu_{j}^{k} \nabla g_{j}\left(x^{k}\right)
$$

e

$$
\sum_{i=1}^{m}\left|\lambda_{i}^{k} h_{i}\left(x^{k}\right)\right|+\sum_{j \in J\left(x^{*}\right)}\left|\mu_{j}^{k} g_{j}\left(x^{k}\right)\right| \leq r^{k} .
$$

De $r^{k} \rightarrow 0$, a expressão 2.4.31 é satisfeita e de $\omega^{k} \rightarrow \omega^{*}, \zeta^{k}:=\nabla f\left(x^{k}\right)+\omega^{k}=-\omega^{*}+\omega^{k} \rightarrow 0$. Portanto, a condição sequencial CAKKT vale em $x^{*}$, $\operatorname{logo}-\nabla f\left(x^{*}\right)=\omega^{*} \in K\left(x^{*}\right)=K_{C}\left(x^{*}, 0\right)$, finalizando a demonstração.

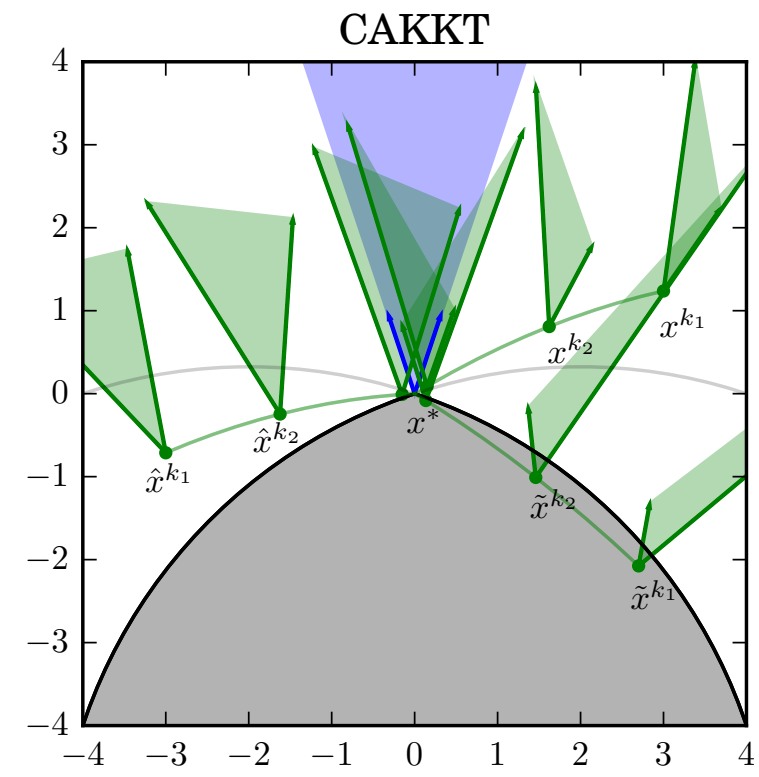

Figura 3.3: Exemplo de cones associados com a condição CAKKT.

Na Figura 3.3, temos um exemplo do cone associados com a condição CAKKT. O conjunto viável é o mesmo da Figura 3.1. Note que a condição CAKKT-regular basicamente diz que todos os possíveis pontos limites de vetores do cone verde devem pertencer ao cone azul, que é o normal do cone tangente linearizado, i.e., $L_{\Omega}^{\circ}\left(x^{*}\right)$. Os conjuntos convexos associados com a condição CAKKT 
são os cones associados com a condição AKKT com a restrição adicional que limita o tamanho dos vetores dependendo de quão perto a zero está a respectiva restrição e quão grande é o parâmetro $r^{k}$. Na Figura 3.3. $\left\{r^{k}\right\}$ converge a 0 com uma velocidade que é proporcional à velocidade das sequências quando se aproximam a $x^{*}$.

Como CAKKT é uma condição de otimalidade, temos o seguinte corolário.

Corolário 3.1.7. A condição CAKKT regular é uma condição de qualificação.

\subsubsection{CQ associada a SAKKT}

Definição 3.1.4. Seja $x^{*} \in \Omega$ um ponto viável. A condição SAKKT regular se cumpre em $x^{*}$ se a multifunção $\mathbb{R}^{n} \ni x \rightrightarrows N_{\Omega(x, 0)}(x)$ é semicontínua exteriormente em $x^{*}$, isto é

$$
\limsup _{x \rightarrow x^{*}} N_{\Omega(x, 0)}(x) \subset N_{\Omega\left(x^{*}, 0\right)}\left(x^{*}\right)=K\left(x^{*}\right) .
$$

Da mesma forma que calculamos o cone normal do conjunto $\Omega(x,-\infty)$, é possível calcular o cone normal de $\Omega(x, 0)$.

Proposição 3.1.8. Sejam $x$ e $\varepsilon \operatorname{com} x+\varepsilon$ em $\Omega(x, 0)$. Então, todo elemento de $N_{\Omega(x, 0)}(x+\varepsilon)$ é da forma

$$
\sum_{i=1}^{m} \lambda_{i} \nabla h_{i}(x)+\sum_{j: g_{j}(x) \geq 0} \mu_{j} \nabla g_{j}(x),
$$

onde $\lambda_{i} \in \mathbb{R}, \mu_{j} \in \mathbb{R}_{+}$e $\mu_{j}\left(\left\langle\nabla g_{j}(x), \varepsilon\right\rangle\right)=0$ sempre que $g_{j}(x) \geq 0$. Além disso, o cone $N_{\Omega(x, 0)}(x+\varepsilon)$ é um subconjunto de $N_{\Omega(x, 0)}(x)$.

Teorema 3.1.9. Seja $x^{*} \in \Omega$. Então, $x^{*}$ é $S A K K T$ regular se, e somente se, a condição sequencial SAKKT em $x^{*}$ implica as condições KKT em $x^{*}$, independentemente da função objetivo.

O resultado do teorema anterior pode ser expressado como: a condição mínima para garantir que a condição sequencial SAKKT em $x^{*}$ implique as condições KKT é a semicontinuidade exterior de $N_{\Omega(x, 0)}(x)$ em $x^{*}$.

Demonstração. Primeiro, provaremos que se $x^{*}$ é um ponto SAKKT regular então a condição sequencial SAKKT em $x^{*}$ implica as condições KKT em $x^{*}$, independentemente da função objetivo. Seja $f$ uma função objetivo tal que a condição SAKKT vale. Usando a equivalência entre a condição AGP $(0)$ e a condição SAKKT, (Sch06, Teorema 1.2.6(c)), temos que existe uma sequência $\left\{x^{k}\right\} \subset$ $\mathbb{R}^{m}$, com $x^{k} \rightarrow x^{*}$ e $P_{\Omega\left(x^{k}, 0\right)}\left(x^{k}-\nabla f\left(x^{k}\right)\right)-x^{k} \rightarrow 0$. Defina $y^{k}:=P_{\Omega\left(x^{k}, 0\right)}\left(x^{k}-\nabla f\left(x^{k}\right)\right)$ e $\varepsilon^{k}:=y^{k}-x^{k}$. Claramente, da condição AGP, $\lim _{k \rightarrow \infty} \varepsilon^{k}=0$. Da Proposição 2.1.2 temos que

$$
\omega^{k}=x^{k}-\nabla f\left(x^{k}\right)-y^{k} \in N_{\Omega\left(x^{k}, 0\right)}\left(y^{k}=x^{k}+\varepsilon^{k}\right) \subset N_{\Omega\left(x^{k}, 0\right)}\left(x^{k}\right),
$$

onde a última inclusão vem da Proposição 3.1.8. Assim, a sequência $\left\{\omega^{k}\right\}$ satisfaz

$$
\omega^{k} \in N_{\Omega\left(x^{k}, 0\right)}\left(x^{k}\right) \text { e } \omega^{k}=x^{k}-\nabla f\left(x^{k}\right)-y^{k} \rightarrow-\nabla f\left(x^{*}\right) .
$$

Da definição de limite exterior e da definição de semi-continuidade exterior temos

$$
-\nabla f\left(x^{*}\right) \in \limsup _{x \rightarrow x^{*}} N_{\Omega(x, 0)}(x) \subset N_{\Omega\left(x^{*}, 0\right)}\left(x^{*}\right)=K\left(x^{*}\right),
$$

provando que $x^{*}$ satisfaz as condições KKT.

Agora provaremos que, se a condição sequencial SAKKT em $x^{*}$ implica as condições KKT em $x^{*}$, independentemente da função objetivo, então $x^{*}$ é um ponto SAKKT regular. A prova segue as mesmas linhas do Teorema 3.1.4. Seja $\omega^{*} \in \lim \sup N_{\Omega(x, 0)}(x)$. Da definição de limite 
exterior existem sequências $\left\{x^{k}\right\}$ e $\left\{\omega^{k}\right\}$ tais que $x^{k} \rightarrow x^{*}, \omega^{k} \rightarrow \omega^{*}$ e $\omega^{k} \in N_{\Omega\left(x^{k}, 0\right)}\left(x^{k}\right)$. Defina $f(x):=-\left\langle w^{*}, x\right\rangle$ para todo $x \in \mathbb{R}^{n}$. Vejamos que a condição $\mathrm{AGP}(0)$ é válida em $x^{*}$ para a função $f(x)=-\left\langle w^{*}, x\right\rangle$. Denote $z^{k}:=P_{\Omega\left(x^{k}, 0\right)}\left(x^{k}-\nabla f\left(x^{k}\right)\right)=P_{\Omega\left(x^{k}, 0\right)}\left(x^{k}+\omega^{*}\right)$. Da não expansividade da projeção $P_{\Omega\left(x^{k}, 0\right)}(x)$ e da relação $P_{\Omega\left(x^{k}, 0\right)}\left(\omega^{k}+x^{k}\right)=x^{k}$ temos que

$$
\left\|z^{k}-x^{k}\right\|=\left\|P_{\Omega\left(x^{k}, 0\right)}\left(x^{k}+\omega^{*}\right)-P_{\Omega\left(x^{k}, 0\right)}\left(\omega^{k}+x^{k}\right)\right\| \leq\left\|\omega^{*}-\omega^{k}\right\| .
$$

A última desigualdade implica a convergência de $z^{k}-x^{k}$ para zero. Assim, a condição AGP(0) (equivalentemente SAKKT) vale em $x^{*}$. Da hipótese, as condições KKT valem em $x^{*}$, isto é, $-\nabla f\left(x^{*}\right)=\omega^{*}$ pertence a $N_{\Omega\left(x^{*}, 0\right)}\left(x^{*}\right)=K\left(x^{*}\right)$, o que conclui a prova do teorema.

Corolário 3.1.10. A condição SAKKT regular é uma condição de qualificação.

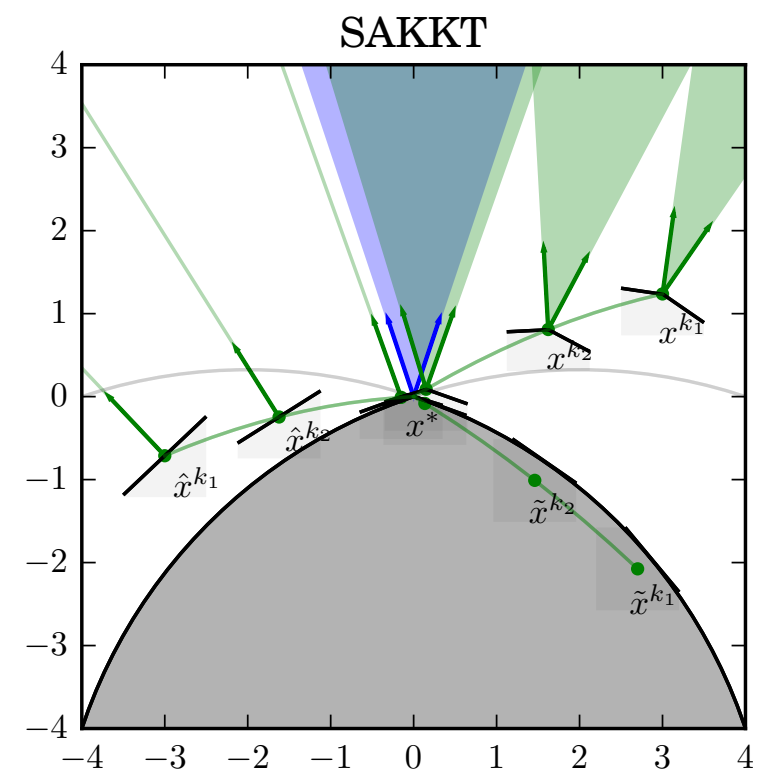

Figura 3.4: Exemplo do cone associado à condição SAKKT

Na Figura 3.4, temos um exemplo do cone associado com a condição SAKKT. O conjunto viável assim como também a região viável é a mesma da Figura 3.1. Note que a condição SAKKT-regular basicamente diz que todos os possíveis pontos limites de vetores do cone verde devem pertencer ao cone azul, que é o normal do cone tangente linearizado, i.e., $L_{\Omega}^{\circ}\left(x^{*}\right)$. Os cones associados à condição SAKKT sempre têm em consideração as restrições que são ativas no ponto e não têm nenhuma perturbação $\varepsilon^{k}$, que é a principal diferença com o cone associado à condição AGP, ver Figura 3.2 .

\subsubsection{CQ associada a L-AGP}

Para esta condição sequencial de otimalidade também temos uma condição de qualificação associada, que apresentamos abaixo:

Definição 3.1.5. Seja $x^{*} \in \Omega$ dizemos que $x^{*}$ satisfaz a condição do $L$-AGP regular se a multifunção

$$
\mathbb{R}^{n} \times \mathbb{R}_{+} \ni(x, \varepsilon) \rightrightarrows N_{\Omega_{N L}(x,-\infty) \cap \Omega_{L}}(x+\varepsilon)
$$

é semicontínua exteriormente relativamente a $\Omega_{L} \times \mathbb{R}^{m}$ no ponto $\left(x^{*}, 0\right)$, isto é,

$$
\limsup _{(x, \varepsilon) \rightarrow\left(x^{*}, 0\right), x \in \Omega_{L}} N_{\Omega_{N L}(x,-\infty) \cap \Omega_{L}}(x+\varepsilon) \subset N_{\Omega_{N L}\left(x^{*},-\infty\right) \cap \Omega_{L}}\left(x^{*}\right)=K\left(x^{*}\right) .
$$

O teorema principal desta subseção é o seguinte 
Teorema 3.1.11. Seja $x^{*} \in \Omega$. L-AGP regular é a condição mínima para garantir que a condição sequencial de otimalidade $L$-AGP implica a condição KKT, independentemente da função objetivo.

Demonstração. A prova segue as mesmas linhas da demonstração do Teorema 3.1.4.

Corolário 3.1.12. A condição L-AGP regular é uma condição de qualificação.

Guignard CQ $\longleftrightarrow$ Mínimo implica KKT

Figura 3.5: Equivalência das novas condições de qualificação com as condições sequenciais.

\subsection{Relações entre as novas condições de qualificação}

O objetivo desta seção é mostrar detalhadamente as relações existentes entre as novas condições de qualificação. O seguinte teorema é consequência das relações conhecidas existentes entre as condições sequênciais de otimalidade AKKT, AGP, L-AGP, SAKKT e CAKKT.

Teorema 3.2.1. Seja $x^{*}$ um ponto viável então temos as seguintes relações:

1. Se a condição CCP vale em $x^{*}$ então $x^{*}$ é um ponto AGP regular;

2. Se $x^{*}$ é AGP regular então $x^{*}$ é um ponto SAKKT regular;

3. Se $x^{*}$ é $A G P$ regular então $x^{*}$ é um ponto $L$-AGP regular;

4. Se $x^{*}$ é SAKKT regular então $x^{*}$ é um ponto CAKKT regular.

As relações são estritas, como mostram os seguintes exemplos:

Exemplo 3.2.1. (AGP regular é estritamente mais fraca que a condição CCP).

Em $\mathbb{R}^{2}$, consideremos $x^{*}=(0,0)^{\top}$ e as restrições de desigualdade definidas por

$$
\begin{aligned}
& g_{1}(x, y)=-x_{1} \\
& g_{2}(x, y)=x_{1}+\left(x_{1}^{+}\right)^{2} \exp \left(x_{2}^{2}\right) .
\end{aligned}
$$

O ponto $x^{*}=(0,0)^{\top}$ é viável e ambas restrições são ativas em $x^{*}$. Além disso, as derivadas são:

$\nabla g_{1}\left(x_{1}, x_{2}\right)=(-1,0)^{T} \quad$ e $\quad \nabla g_{2}\left(x_{1}, x_{2}\right)=\left(1+2 x_{1}^{+} \exp \left(x_{2}^{2}\right), 2 x_{2}\left(x_{1}^{+}\right)^{2} \exp \left(x_{2}^{2}\right)\right)^{\top} \forall x=\left(x_{1}, x_{2}\right)^{\top} \in \mathbb{R}^{2}$.

Assim, temos que o cone $K\left(x^{*}\right)=\left\{\mu_{1}(-1,0)^{\top}+\mu_{2}(1,0)^{\top}: \mu_{1}, \mu_{2} \geq 0\right\}=\mathbb{R} \times\{0\}$. 
A condição CCP não é satisfeita em $x^{*}=(0,0)^{\top}$.

Defina $x^{k}:=1 / k, y^{k}:=1 / k, \mu_{2}^{k}:=\left(2 x_{2}^{k}\left(x_{1}^{k}\right)^{2} \exp \left(x_{2}^{k}\right)^{2}\right)^{-1}$ e $\mu_{1}^{k}:=\mu_{2}^{k}+\left(x_{1}^{k} x_{2}^{k}\right)^{-1}$. Claramente, $\left(x_{1}^{k}, x_{2}^{k}\right)^{\top} \rightarrow(0,0)^{\top}$ e $\mu_{1}^{k}, \mu_{2}^{k} \geq 0$. Assim

$$
\omega^{k}:=\mu_{1}^{k}(-1,0)^{\top}+\mu_{2}^{k}\left(1+2\left(x_{1}^{k}\right)^{+} \exp \left(x_{2}^{k}\right)^{2}, 2 x_{2}^{k}\left(x_{1}^{k}\right)^{2} \exp \left(x_{2}^{k}\right)^{2}\right)^{\top} \in K\left(\left(x_{1}^{k}, x_{2}^{k}\right)\right) .
$$

Das escolhas dos multiplicadores temos que $\omega^{k}=(0,1)^{\top} \forall k \in \mathbb{N}$, portanto $(0,1)^{\top} \in \lim \sup _{x \rightarrow x^{*}} K(x)$ mas $(0,1)^{\top}$ não pertence a $K\left(x^{*}\right)$, assim $K(x)$ não é semicontínuo exteriormente em $x^{*}$.

$O$ ponto $x^{*}=(0,0)^{\top}$ satisfaz a condição AGP regular.

Seja $\omega^{*}=\left(\omega_{1}, \omega_{2}\right)^{\top} \in \lim \sup _{(x, \varepsilon) \rightarrow\left(x^{*}, 0\right)} N_{\Omega(x,-\infty)}(x+\varepsilon)$. Da definição de limite exterior existem sequências $\left\{x^{k}=\left(x_{1}^{k}, x_{2}^{k}\right)^{\top}\right\},\left\{\omega^{k}\right\}$ e $\left\{\varepsilon^{k}=\left(\varepsilon_{1}^{k}, \varepsilon_{2}^{k}\right)^{z t o p}\right\}$ em $\mathbb{R}^{2}$ tais que $x^{k} \rightarrow x^{*}, \varepsilon^{k} \rightarrow(0,0)$, $\omega^{k} \rightarrow \omega^{*}$ e $\omega^{k} \in N_{\Omega\left(x^{k},-\infty\right)}\left(x^{k}+\varepsilon^{k}\right)$. Para provar que $\omega^{*} \in N_{\Omega\left(x^{*},-\infty\right)}\left(x^{*}\right)=K\left(x^{*}\right)$ analisaremos os diversos casos possíveis $\left(x_{1}^{k}>0, x_{1}^{k}<0\right.$ e $\left.x_{1}^{k}=0\right)$.

Existem infinitos índices $k \in \mathbb{N}$ tais que

1. Caso $x_{1}^{k}>0$. Neste caso temos que $g_{1}\left(x_{1}^{k}, x_{2}^{k}\right)<0$ e $g_{2}\left(x_{1}^{k}, x_{2}^{k}\right)>0$. Para este caso definimos duas condições

$$
\begin{aligned}
& \text { condição }\left(g_{1}\right) \text { se } g_{1}\left(x_{1}^{k}, x_{2}^{k}\right)+\left\langle\nabla g_{1}\left(x_{1}^{k}, x_{2}^{k}\right),\left(\varepsilon_{1}^{k}, \varepsilon_{2}^{k}\right)^{\top}\right\rangle=0 . \\
& \text { condiçãa }\left(g_{2}\right) \text { se }\left\langle\nabla g_{2}\left(x_{1}^{k}, x_{2}^{k}\right),\left(\varepsilon_{1}^{k}, \varepsilon_{2}^{k}\right)^{\top}\right\rangle=0 .
\end{aligned}
$$

Dependendo se $\varepsilon^{k}=\left(\varepsilon_{1}^{k}, \varepsilon_{2}^{k}\right)^{\top}$ satisfaz a condição $\left(g_{1}\right)$ ou a condição $\left(g_{2}\right)$ temos os seguintes sub-casos:

(a) Se $\varepsilon^{k}$ satisfaz a condição $\left(g_{1}\right)$ e a condição $\left(g_{2}\right)$.

Como $\varepsilon^{k}$ satisfaz as condições $\left(g_{1}\right)$ e $\left(g_{2}\right)$ temos que

$$
\varepsilon_{x}^{k}=-x_{1}^{k}=-\left(x_{1}^{k}\right)^{+} \text {e } \varepsilon_{1}^{k}\left(1+2\left(x_{1}^{k}\right)^{+} \exp \left(x_{2}^{k}\right)^{2}\right)+\varepsilon_{2}^{k}\left(2 x_{2}^{k}\left(x_{1}^{k}\right)^{2} \exp \left(x_{2}^{k}\right)^{2}\right)=0 .
$$

Usando (3.2.2) obtemos

$$
-1-2 x_{1}^{k} \exp \left(x_{2}^{k}\right)^{2}+\varepsilon_{2}^{k}\left(2 x_{2}^{k} x_{1}^{k} \exp \left(x_{2}^{k}\right)^{2}\right)=0 .
$$

Tomando limites na última expressão (3.2.3 chegamos a uma contradição, assim não podem existir infinitos índices tais que $\varepsilon^{k}$ satisfaz a condição $\left(g_{1}\right)$ e a condição $\left(g_{2}\right)$ simultaneamente;

(b) Se $\varepsilon^{k}$ satisfaz a condição $\left(g_{1}\right)$ mas não a condição $\left(g_{2}\right)$.

Como $\omega^{k} \in N_{\Omega\left(x^{k},-\infty\right)}\left(x^{k}+\varepsilon^{k}\right)$ e $\varepsilon^{k}$ não satisfaz a condição $\left(g_{2}\right)$ temos que o multiplicador associado a $\nabla g_{2}\left(x^{k}, y^{k}\right)$ é nulo, ver Proposição 3.1.3. Assim $\omega^{k}=\mu_{1}^{k}(-1,0)^{\top} \in \mathbb{R} \times\{0\}$ para algum $\mu_{1}^{k} \geq 0$, logo o limite $\omega^{*}$ pertencer a $\mathbb{R} \times\{0\} ;$

(c) Se $\varepsilon^{k}$ não satisfaz a condição $\left(g_{1}\right)$ mas satisfaz a condição $\left(g_{2}\right)$.

Como $\varepsilon^{k}$ não satisfaz a condição $\left(g_{1}\right)$, o multiplicador associado a $\nabla g_{1}\left(x^{k}, y^{k}\right)$ é nulo, veja a Proposição 3.1.3. Assim

$$
\omega^{k}:=\mu_{2}^{k}\left(1+2 x_{1}^{k} \exp \left(x_{2}^{k}\right)^{2}, 2 x_{2}^{k}\left(x_{1}^{k}\right)^{2} \exp \left(x_{2}^{k}\right)^{2}\right)^{\top} \text { para algum } \mu_{2}^{k} \geq 0 .
$$

Além disso, da condição $\left(g_{2}\right)$ temos que

$$
\varepsilon_{1}^{k}\left(1+2\left(x_{1}^{k}\right)^{+} \exp \left(x_{2}^{k}\right)^{2}\right)+\varepsilon_{2}^{k}\left(2 x_{2}^{k}\left(\left(x_{1}^{k}\right)^{+}\right)^{2} \exp \left(x_{2}^{k}\right)^{2}\right)=0 .
$$

Mostraremos que $\omega_{2}^{*}=\lim _{k \rightarrow \infty} \omega_{2}^{k}$ é zero. Suponha, por contradição, que $\omega_{2}^{*}$ é diferente de zero. Logo, para $k$ suficientemente grande, temos

$$
\mu_{2}^{k}\left|2 x_{2}^{k}\left(x_{1}^{k}\right)^{2} \exp \left(x_{2}^{k}\right)^{2}\right|>(1 / 2)\left|\omega_{2}^{*}\right|>0 .
$$


Como consequência $x_{2}^{k}$ é positivo e

$$
\mu_{2}^{k}>\frac{1}{2} \frac{\left|\omega_{2}^{*}\right|}{\left|2 x_{2}^{k}\left(x_{1}^{k}\right)^{2} \exp \left(x_{2}^{k}\right)^{2}\right|} .
$$

Multiplicando (3.2.5 por $\left(1+2 x_{1}^{k} \exp \left(x_{2}^{k}\right)^{2}\right)$ temos que

$$
\omega_{1}^{k}=\mu_{2}^{k}\left(1+2 x_{1}^{k} \exp \left(x_{2}^{k}\right)^{2}\right)>\frac{\left|\omega_{2}^{*}\right|}{2}\left(\frac{1+2 x_{1}^{k} \exp \left(x_{2}^{k}\right)^{2}}{\left|2 x_{2}^{k}\left(x_{1}^{k}\right)^{2} \exp \left(x_{2}^{k}\right)^{2}\right|}\right)>\frac{\left|\omega_{2}^{*}\right|}{2\left|x_{1}^{k} x_{2}^{k}\right|} .
$$

Tomando limites para uma subsequência adequada chegamos a uma contradição, porque o lado esquerdo converge para $\omega_{1}^{*}$ enquanto o lado direito vai para o infinito.

(d) Se $\varepsilon^{k}$ não satisfaz nem a condição $\left(g_{1}\right)$ nem a condição $\left(g_{2}\right)$.

Como $\varepsilon^{k}$ não satisfaz nenhuma das condições $\left(g_{1}\right)$ ou $\left(g_{2}\right)$ temos que os multiplicadores associados a $\nabla g_{1}\left(x^{k}, y^{k}\right)$ e $\nabla g_{2}\left(x^{k}, y^{k}\right)$ são nulos e portanto $\omega^{k}=(0,0)^{\top} \in \mathbb{R} \times\{0\}$.

Assim, se existem infinitos índices $k$ que satisfazem o caso $\left(x_{1}^{k}>0\right)$, tomando subsequências adequadas, temos que $\omega^{*} \in \mathbb{R} \times\{0\}$;

2. Caso $x_{1}^{k}<0$. Neste caso, $g_{1}\left(x_{1}^{k}, x_{2}^{k}\right)>0$ e $g_{2}\left(x_{1}^{k}, x_{2}^{k}\right)<0$.

Para este caso definimos duas condições

$$
\begin{aligned}
& \text { condição }\left(g_{1}\right) \text { se }\left\langle\nabla g_{1}\left(x_{1}^{k}, x_{2}^{k}\right),\left(\varepsilon_{1}^{k}, \varepsilon_{2}^{k}\right)^{\top}\right\rangle=0 \\
& \text { condição }\left(g_{2}\right) \text { se } g_{2}\left(x_{1}^{k}, x_{2}^{k}\right)+\left\langle\nabla g_{2}\left(x_{1}^{k}, x_{2}^{k}\right),\left(\varepsilon_{1}^{k}, \varepsilon_{2}^{k}\right)^{\top}\right\rangle=0 .
\end{aligned}
$$

Dependendo se $\varepsilon^{k}$ satisfaz a condição $\left(g_{1}\right)$ ou a condição $\left(g_{2}\right)$ temos os seguintes sub-casos:

(a) Se $\varepsilon^{k}$ satisfaz a condição $\left(g_{1}\right)$ e a condição $\left(g_{2}\right)$.

Das condições $\left(g_{1}\right)$ e $\left(g_{2}\right)$ temos que

$$
\varepsilon_{1}^{k}=0 \text { e } x_{1}^{k}+\left(\left(x_{1}^{k}\right)^{+}\right)^{2} \exp x_{2}^{2}+\varepsilon_{1}^{k}\left(1+2\left(x_{1}^{k}\right)^{+} \exp \left(x_{2}^{k}\right)^{2}\right)+\varepsilon_{2}^{k}\left(2 x_{2}^{k}\left(\left(x_{1}^{k}\right)^{+}\right)^{2} \exp \left(x_{2}^{k}\right)^{2}\right)=0 .
$$

Usando o fato que $\left(x_{1}^{k}\right)^{+}=0$ e $\varepsilon_{1}^{k}=0$, a última igualdade implica que $x_{1}^{k}=0$, claramente, uma contradição $\operatorname{com} x_{1}^{k}<0$;

(b) Se $\varepsilon^{k}$ satisfaz a condição $\left(g_{1}\right)$ mas não a condição $\left(g_{2}\right)$.

Como $\varepsilon^{k}$ satisfaz a condição $\left(g_{1}\right), \varepsilon_{1}^{k}=0$ e como $\varepsilon^{k}$ não satisfaz a condição $\left(g_{2}\right)$ o multiplicador associado a $\nabla g_{2}\left(x^{k}, y^{k}\right)$ é nulo, $\operatorname{logo} \omega^{k}=\mu_{1}^{k}(-1,0)$ para algum $\mu_{1}^{k}$ não negativo. Tomando limite, concluímos que $\omega^{*}$ pertencer a $\mathbb{R} \times\{0\}$;

(c) Se $\varepsilon^{k}$ não satisfaz a condição $\left(g_{1}\right)$ e satisfaz a condição $\left(g_{2}\right)$.

Como $\varepsilon^{k}$ não satisfaz a condição $\left(g_{1}\right)$, o multiplicador associado a $\nabla g_{1}\left(x^{k}, y^{k}\right)$ é zero, Proposição 3.1.3, e portanto

$$
\omega^{k}=\mu_{2}^{k}\left(1+2\left(x_{1}^{k}\right)^{+} \exp \left(x_{2}^{k}\right)^{2}, 2 x_{2}^{k}\left(\left(x_{1}^{k}\right)^{+}\right)^{2} \exp \left(x_{2}^{k}\right)^{2}\right)^{\top}=\mu_{2}^{k}(1,0)^{\top}
$$

e

$x_{1}^{k}+\left(x_{+}^{k}\right)^{2} \exp x_{2}^{2}+\varepsilon_{1}^{k}\left(1+2\left(x_{1}^{k}\right)^{+} \exp \left(x_{2}^{k}\right)^{2}\right)+\varepsilon_{2}^{k}\left(2 x_{2}^{k}\left(\left(x_{1}^{k}\right)^{+}\right)^{2} \exp \left(x_{2}^{k}\right)^{2}\right)=x_{1}^{k}+\varepsilon_{1}^{k}=0$.

Tomando o limite para uma subsequência adequada, vemos que $\omega^{*} \in \mathbb{R} \times\{0\}$;

(d) Se $\varepsilon^{k}$ não satisfaz a condição $\left(g_{1}\right)$ e não satisfaz a condição $\left(g_{2}\right)$.

Como $\varepsilon^{k}$ não satisfaz nenhuma condição, os multiplicadores associados a cada $\nabla g_{1}\left(x^{k}, y^{k}\right)$

e $\nabla g_{2}\left(x^{k}, y^{k}\right)$ são nulos e como consequência $\omega^{k}=(0,0)^{\top}$. 
Portanto, se existem infinitos índices $k$ que satisfazem este caso $\left(x_{1}^{k}<0\right)$, tomando subsequências adequadas, o limite $\omega^{*}$ está em $\mathbb{R} \times\{0\}$;

3. Caso $x_{1}^{k}=0$. Neste caso temos que $g_{1}\left(x_{1}^{k}, x_{2}^{k}\right)=0$ e $g_{2}\left(x_{1}^{k}, x_{2}^{k}\right)=0$. Analogamente ao que fizemos anteriormente, definimos duas condições

$$
\begin{aligned}
& \text { condição }\left(g_{1}\right) \text { se }\left\langle\nabla g_{1}\left(x_{1}^{k}, x_{2}^{k}\right),\left(\varepsilon_{1}^{k}, \varepsilon_{2}^{k}\right)^{\top}\right\rangle=0 \\
& \text { condição }\left(g_{2}\right) \text { se }\left\langle\nabla g_{2}\left(x_{1}^{k}, x_{2}^{k}\right),\left(\varepsilon_{1}^{k}, \varepsilon_{2}^{k}\right)^{\top}\right\rangle=0 .
\end{aligned}
$$

Dependendo se $\varepsilon^{k}$ satisfaz a condição $\left(g_{1}\right)$ ou a condição $\left(g_{2}\right)$ temos os seguintes sub-casos:

(a) Se $\varepsilon^{k}$ satisfaz a condição $\left(g_{1}\right)$ e a condição $\left(g_{2}\right)$.

Neste caso

$$
\omega^{k}=\mu_{1}^{k}(-1,0)^{\top}+\mu_{2}^{k}\left(1+2\left(x_{1}^{k}\right)^{+} \exp \left(x_{2}^{k}\right)^{2}, 2 x_{2}^{k}\left(\left(x_{1}^{k}\right)^{+}\right)^{2} \exp \left(x_{2}^{k}\right)^{2}\right)^{\top}=\mu_{1}^{k}(-1,0)^{\top}+\mu_{2}^{k}(1,0)^{\top},
$$

para alguns $\mu_{1}^{k}, \mu_{2}^{k} \geq 0$. Assim, $\omega^{k} \in \mathbb{R} \times\{0\}$.

(b) Se $\varepsilon^{k}$ satisfaz a condição $\left(g_{1}\right)$ mas não a condição $\left(g_{2}\right)$.

Como $\varepsilon^{k}$ não satisfaz a condição $\left(g_{2}\right)$, o multiplicador associado a $\nabla g_{2}$ é zero, assim existe algum $\mu_{1}^{k} \geq 0$ tal que $\omega^{k}=\mu_{1}^{k}(-1,0)^{\top} \in \mathbb{R} \times\{0\}$.

(c) Se $\varepsilon^{k}$ não satisfaz a condição $\left(g_{1}\right)$ mas satisfaz a condição $\left(g_{2}\right)$.

Como $\varepsilon^{k}$ não satisfaz a condição $\left(g_{1}\right)$ o multiplicador associado é nulo e existe algum $\mu_{2}^{k} \geq 0$ tal que

$$
\omega^{k}=\mu_{2}^{k}\left(1+2\left(x_{1}^{k}\right)^{+} \exp \left(x_{2}^{k}\right)^{2}, 2 x_{2}^{k}\left(\left(x_{1}^{k}\right)^{+}\right)^{2} \exp \left(x_{2}^{k}\right)^{2}\right)^{\top}=\mu_{2}^{k}(1,0)^{\top} \in \mathbb{R} \times\{0\} .
$$

(d) Se $\varepsilon^{k}$ não satisfaz a condição $\left(g_{1}\right)$ nem satisfaz a condição $\left(g_{2}\right)$.

Não existe multiplicador não nulo associado a $\nabla g_{1}\left(x_{1}^{k}, x_{2}^{k}\right)$ e $\nabla g_{2}\left(x_{1}^{k}, x_{2}^{k}\right)$, logo $\omega^{k}=$ $(0,0)^{\top}$.

Assim, se existem infinitos índices $k$ que satisfazem $x^{k}=0$, tomando subsequências adequadas, temos que $\omega^{*} \in \mathbb{R} \times\{0\}$.

De todos os casos analisados temos que $\omega^{*}=\lim _{k \rightarrow \infty} \omega^{k}$ necessariamente pertence a $\mathbb{R} \times\{0\}=$ $N_{\Omega\left(x^{*},-\infty\right)}\left(x^{*}\right)$, o que implica que $x^{*}$ é um ponto AGP regular.

Exemplo 3.2.2. (SAKKT regular é estritamente mais fraca que AGP regular).

Em $\mathbb{R}^{2}$ considere o ponto $x^{*}=(0,0)^{\top}$ e as restrições de desigualdade definidas por

$$
\begin{aligned}
& g_{1}\left(x_{1}, x_{2}\right)=-x_{1} \\
& g_{2}\left(x_{1}, x_{2}\right)=-x_{1}^{2}-x_{2}^{2} .
\end{aligned}
$$

O ponto $x^{*}=(0,0)^{\top}$ é um ponto viável e para ambas restrições nas ativas em $x^{*}$. As derivadas são:

$$
\nabla g_{1}\left(x_{1}, x_{2}\right)=(-1,0)^{\top} \quad \text { e } \nabla g_{2}\left(x_{1}, x_{2}\right)=\left(-2 x_{1},-2 x_{2}\right)^{\top} \forall x=\left(x_{1}, x_{2}\right)^{\top} \in \mathbb{R}^{2} .
$$

Da forma das derivadas temos que, $K\left(x^{*}\right)=\left\{\mu_{1}(-1,0)^{\top}+\mu_{2}(0,0)^{\top}: \mu_{1}, \mu_{2} \geq 0\right\}=\mathbb{R}_{-} \times\{0\}$. Agora vejamos que a condição SAKKT regular vale e a condição AGP regular falha.

A condição SAKKT regular vale em $x^{*}$.

Temos que provar que a multifunção $N_{\Omega(x, 0)}(x)$ é semicontínua exteriormente em $x^{*}=(0,0)^{\top}$. Seja $\omega^{*}=\left(\omega_{1}^{*}, \omega_{2}^{*}\right)^{\top} \in \lim \sup _{x \rightarrow x^{*}} N_{\Omega(x, 0)}(x)$. Da definição de limite exterior, existem sequências $\left\{x^{k}=\left(x_{1}^{k}, x_{2}^{k}\right)^{\top}\right\}$ e $\left\{\omega^{k}\right\}$ em $\mathbb{R}^{2}$ tais que $x^{k} \rightarrow x^{*}, \omega^{k} \rightarrow \omega^{*}$ e $\omega^{k} \in N_{\Omega\left(x^{k}, 0\right)}\left(x^{k}\right)$. 
Para provar que $\omega^{*} \in N_{\Omega\left(x^{*},-\infty\right)}\left(x^{*}\right)=K\left(x^{*}\right)$ analisaremos os diversos casos possíveis, da mesma forma como fizemos com o exemplo anterior. Suponha que existem infinitos índices $k \in \mathbb{N}$ tais que algum dos seguintes casos sejam verdadeiros.

1. Se $x_{1}^{k}>0$. Neste caso $g_{1}\left(x_{1}^{k}, x_{2}^{k}\right)<0$ e $g_{2}\left(x_{1}^{k}, x_{2}^{k}\right)<0$. Da Proposição 3.1.8 temos que $\omega^{k}=(0,0)^{\top}$ porque os multiplicadores associados aos gradientes $\nabla g_{1}\left(x_{1}^{k}, x_{2}^{k}\right)$ e $\nabla g_{1}\left(x_{1}^{k}, x_{2}^{k}\right)$ são diferentes de zero apenas quando $g_{1}\left(x_{1}^{k}, x_{2}^{k}\right) \geq 0$ ou $g_{2}\left(x_{1}^{k}, x_{2}^{k}\right) \geq 0$.

2. Se $x_{1}^{k}<0$. Neste caso $g_{1}\left(x_{1}^{k}, x_{2}^{k}\right)>0$ e $g_{2}\left(x_{1}^{k}, x_{2}^{k}\right)<0$. Usando a Proposição 3.1.8, temos que o multiplicador associado a $\nabla g_{2}$ é nulo e portanto $\omega^{k}$ é da forma da $\mu_{1}^{k}(-1,0) \in \mathbb{R}_{-} \times\{0\}$ para algum $\mu_{1}^{k} \geq 0$;

3. Se $x^{k}=0$. Ambas funções são não negativas e dependendo de $x_{2}^{k}$ temos que $g_{2}\left(x_{1}^{k}, x_{2}^{k}\right)$ é estritamente negativo ou igual a zero. Temos os seguintes sub-casos:

(a) Se $x_{2}^{k}=0$, temos que $g_{2}\left(x_{1}^{k}, x_{2}^{k}\right)=0$ e assim

$$
\omega^{k}=\mu_{1}^{k}(-1,0)^{\top}+\mu_{2}^{k}\left(-2 x_{1}^{k},-2 x_{2}^{k}\right)^{\top}=\mu_{1}^{k}(-1,0)^{\top}+\mu_{2}^{k}(0,0)^{\top} \in \mathbb{R}_{-} \times\{0\} .
$$

(b) Se $x_{2}^{k}$ não é zero, temos que $g_{2}\left(x_{1}^{k}, x_{2}^{k}\right)<0$. Da Proposição 3.1 .8 , o multiplicador associado a $g_{2}\left(x_{1}^{k}, x_{2}^{k}\right)$ é igual a zero. Logo, existe $\mu_{1}^{k} \geq 0$ tal que $\omega^{k}=\mu_{1}^{k}(-1,0)^{\top} \in \mathbb{R}_{-} \times\{0\}$.

De todos os casos concluímos que $\omega^{*}$ sempre pertence a $K\left(x^{*}\right)=\mathbb{R}_{-} \times\{0\}$ e como consequência a condição SAKKT regular vale em $x^{*}$.

A condição AGP regular não vale em $x^{*} \in \Omega$.

Para cada $k \in \mathbb{N}$, defina $x_{1}^{k}:=1 / k, x_{2}^{k}:=1 / k, \varepsilon_{1}^{k}:=-x_{1}^{k}, \varepsilon_{2}^{k}:=0$ e multiplicadores $\mu_{2}^{k}:=$ $\left(2 x_{1}^{k}\right)^{-1}$ e $\mu_{1}^{k}:=0$. Defina $\omega^{k}:=\mu_{1}^{k}(-1,0)^{\top}+\mu_{2}^{k}\left(-2 x_{1}^{k},-2 x_{2}^{k}\right)^{\top}$. Claramente, temos que $\varepsilon^{k} \rightarrow 0$ e $\left(x_{1}^{k}, x_{2}^{k}\right) \rightarrow(0,0)$. Da Proposição 3.1.3 obtemos que $\omega^{k} \in N_{\Omega\left(\left(x_{1}^{k}, x_{2}^{k}\right),-\infty\right)}\left(\left(x_{1}^{k}, x_{2}^{k}\right)+\left(\varepsilon_{1}^{k}, \varepsilon_{2}^{k}\right)\right)$. Ainda mais, da escolha dos multiplicadores $\mu_{1}^{k}$ e $\mu_{2}^{k}$, temos que $\omega^{k}=(-1,-1)^{\top} \forall k \in \mathbb{N}$ e portanto $(-1,-1)^{\top} \in \lim \sup _{(x, \varepsilon) \rightarrow\left(x^{*}, 0\right)} N_{\Omega(x,-\infty)}(x+\varepsilon)$ mas $(-1,-1)^{\top}$ não pertence a $K\left(x^{*}\right)=\mathbb{R}_{-} \times\{0\}$. Portanto, $x^{*}=(0,0)$ não é um ponto AGP regular.

Exemplo 3.2.3. ( $L-A G P$ regular é estritamente mais fraca que AGP regular).

Em $\mathbb{R}^{2}$, considere $x^{*}=(0,0)^{\top}$ e as restrições de igualdade e desigualdade definidas pelas funções

$$
\begin{aligned}
& h\left(x_{1}, x_{2}\right)=x_{1} ; \\
& g\left(x_{1}, x_{2}\right)=x_{1}-x_{1}^{2} x_{2} .
\end{aligned}
$$

Claramente, $x^{*}=(0,0)^{\top}$ é um ponto viável no qual a restrição de desigualdade é ativa. Calculando as derivadas das restrições

$$
\nabla h\left(x_{1}, x_{2}\right)=(1,0)^{\top} \text { e } \nabla g\left(x_{1}, x_{2}\right)=\left(1-2 x_{1} x_{2},-x_{1}^{2}\right)^{\top} \text { para todo }\left(x_{1}, x_{2}\right)^{\top} \in \mathbb{R}^{2} .
$$

Assim, o cone linearizado é $K\left(x^{*}\right)=\left\{\lambda(1,0)^{\top}+\mu(1,0)^{\top}: \lambda \in \mathbb{R}, \mu \geq 0\right\}=\mathbb{R} \times\{0\}$.

$A$ condição de qualificação $L$-AGP regular é satisfeita em $x^{*}=(0,0)^{\top}$.

Primeiro, vejamos qual é o conjunto das restrições lineares $\Omega_{L}$. Como a única restrição linear é dada pela função $h$ (restrição de igualdade) temos que

$$
\Omega_{L}=\left\{x=\left(x_{1}, x_{2}\right)^{\top} \in \mathbb{R}^{2}: h(x)=0\right\}=\left\{x=\left(x_{1}, x_{2}\right)^{\top} \in \mathbb{R}^{2}: x_{1}=0\right\}=\{0\} \times \mathbb{R} .
$$

Vamos provar que $N_{\Omega_{N L}(x,-\infty) \cap \Omega_{L}}(x+\varepsilon)$ é semicontínua exteriormente em $\left(x^{*}, 0\right)$ relativamente a $\Omega_{L} \times \mathbb{R}^{2}$. Seja $\omega^{*}=\left(\omega_{1}, \omega_{2}\right)^{\top} \in \lim \sup N_{\Omega_{N L}(x,-\infty) \cap \Omega_{L}}(x+\varepsilon)$ relativo a $\Omega_{L} \times \mathbb{R}^{2}$. Então, da definição de limite exterior, existem sequências $\left\{x^{k}\right\},\left\{\omega^{k}\right\}$ e $\left\{\varepsilon^{k}\right\}$ em $\mathbb{R}^{2}$ tais que $x^{k} \rightarrow x^{*}, \varepsilon^{k} \rightarrow$ $(0,0), \omega^{k} \rightarrow \omega^{*} \mathrm{e}$

$$
x^{k} \in \Omega_{L} \quad, \quad x^{k}+\varepsilon^{k} \in \Omega_{N L}\left(x^{k},-\infty\right) \cap \Omega_{L}, \omega^{k} \in N_{\Omega_{N L}\left(x^{k},-\infty\right) \cap \Omega_{L}}\left(x^{k}+\varepsilon^{k}\right) .
$$


Para provar que $\omega^{*} \in N_{\Omega_{N L}\left(x^{*},-\infty\right) \cap \Omega_{L}}\left(x^{*}+0\right)=K\left(x^{*}\right)$ analisaremos os diversos casos possíveis. Como $x^{k} \in \Omega_{L}$ e $x^{k}+\varepsilon^{k} \in \Omega_{L}$ temos que $x_{1}^{k}=0$ e $\varepsilon_{1}^{k}=0$ e como consequência $g\left(x_{1}^{k}, x_{2}^{k}\right)=0$ para todo $k \in \mathbb{N}$. Percebamos que, independentemente do valor de $\epsilon_{2}^{k}$ se satisfaz a condição

$$
\text { condição }(g): \quad \text { se }\left\langle\nabla g\left(x_{1}^{k}, x_{2}^{k}\right),\left(\varepsilon_{1}^{k}, \varepsilon_{2}^{k}\right)^{\top}\right\rangle=0 .
$$

Basta notar que quando $x_{1}^{k}=\varepsilon_{1}^{k}=0$,

$$
\left\langle\nabla g\left(x_{1}^{k}, x_{2}^{k}\right),\left(\varepsilon_{1}^{k}, \varepsilon_{2}^{k}\right)^{\top}\right\rangle=\varepsilon_{1}^{k}\left(1-2 x_{1}^{k} x_{2}^{k}\right)+\varepsilon_{2}^{k}\left(-\left(x_{1}^{k}\right)^{2}\right)=0 .(1-0)+\varepsilon_{2}^{k} \cdot 0=0 .
$$

Como a condição $(g)$ é satisfeita temos que existem $\lambda^{k}$ e $\mu^{k} \geq 0$ (não necessariamente nulos) tais que

$$
\omega^{k}=\lambda^{k} \nabla h\left(x_{1}^{k}, x_{2}^{k}\right)+\mu^{k} \nabla g\left(x_{1}^{k}, x_{2}^{k}\right) \in N_{\Omega_{N L}\left(x^{k},-\infty\right) \cap \Omega_{L}}\left(x^{k}+\varepsilon^{k}\right) .
$$

De $x^{k}=0$ temos que $\nabla h\left(x_{1}, x_{2}\right)=(1,0)^{\top}$ e $\nabla g\left(x_{1}^{k}, x_{2}^{k}\right)=(1,0)^{\top}$, logo $\omega^{k} \in \mathbb{R} \times\{0\}$ para todo $k \in \mathbb{N}$, o que implica que $\omega^{*}=\lim _{k \rightarrow \infty} \omega^{k} \in \mathbb{R} \times\{0\}=K\left(x^{*}\right)$, provando que a condição de qualificação L-AGP é satisfeita em $x^{*}=(0,0)^{\top}$.

$A$ condição de qualificação AGP regular não é satisfeita em $x^{*}=(0,0)^{\top}$.

Escolha $x_{1}^{k}:=1 / k, x_{2}^{k}:=x_{1}^{k}, \epsilon_{1}^{k}:=x_{2}^{k}\left(x_{1}^{k}\right)^{2}$ e $\epsilon_{2}^{k}=x_{2}^{k}\left(1-2 x_{1}^{k} x_{2}^{k}\right)$. Claramente, $\left\|\varepsilon^{k}\right\| \rightarrow 0, g\left(x_{1}^{k}, x_{2}^{k}\right)=$ $x_{1}^{k}\left(1-x_{1}^{k} x_{2}^{k}\right)>0$ (para $k$ suficientemente grande) e o multiplicador associado a $\nabla g\left(x_{1}^{k}, x_{2}^{k}\right)$ é livre já que

$$
\begin{aligned}
\left\langle\nabla g\left(x_{1}^{k}, x_{2}^{k}\right),\left(\varepsilon_{1}^{k}, \varepsilon_{2}^{k}\right)^{\top}\right\rangle & =\varepsilon_{1}^{k}\left(1-2 x_{1}^{k} x_{2}^{k}\right)+\varepsilon_{2}^{k}\left(-\left(x_{1}^{2}\right)^{k}\right) \\
& =x_{2}^{k}\left(x_{1}^{k}\right)^{2}\left(1-2 x_{1}^{k} x_{2}^{k}\right)+x_{2}^{k}\left(1-2 x_{1}^{k} x_{2}^{k}\right)\left(-\left(x_{1}^{2}\right)^{k}\right)=0 .
\end{aligned}
$$

Escolha $\mu^{k}:=\left(\left(x_{1}^{k}\right)^{2}\right)^{-1} \in \mathbb{R}_{+}, \lambda^{k}:=-\mu^{k}\left(1-2 x_{1}^{k} x_{2}^{k}\right)$ e defina a sequência $\left\{\omega^{k}\right\}$ como

$$
\omega^{k}:=\lambda^{k} \nabla h\left(x_{1}^{k}, x_{2}^{k}\right)+\mu^{k} \nabla g\left(x_{1}^{k}, x_{2}^{k}\right)=\lambda^{k}(1,0)^{\top}+\mu^{k}\left(1-2 x_{1}^{k} x_{2}^{k},-\left(x_{1}^{k}\right)^{2}\right)^{\top}=(0,-1)^{\top} .
$$

Da Proposição 3.1.8, temos que $\omega^{k}=(0,-1)^{\top} \in N_{\Omega\left(x^{k},-\infty\right)}\left(x^{k}+\varepsilon^{k}\right)$ para todo $k \in \mathbb{N}$. Assim, $\lim _{k \rightarrow \infty} \omega^{k}=(0,-1)^{\top}$ pertence a $\lim \sup _{(x, \varepsilon) \rightarrow\left(x^{*}, 0\right)} N_{\Omega(x,-\infty)}(x+\varepsilon)$. Mas o vetor $(0,-1)^{\top}$ não pode pertencer a $K\left(x^{*}\right)=\mathbb{R} \times\{0\}$, logo, a condição AGP regular não vale em $x^{*}=(0,0)^{\top}$.

Exemplo 3.2.4. (CAKKT regular não implica SAKKT regular).

Em $\mathbb{R}^{2}$ considere $x^{*}=(0,0)^{\top}$ e as restrições de igualdade e desigualdade definidas pelas funções

$$
\begin{aligned}
& h\left(x_{1}, x_{2}\right)=x_{1} ; \\
& g\left(x_{1}, x_{2}\right)=x_{1} \exp x_{2} .
\end{aligned}
$$

O vetor $x^{*}=(0,0)$ é um ponto viável no qual a restrição de desigualdade é ativa. As derivadas são:

$$
\nabla h\left(x_{1}, x_{2}\right)=(1,0)^{\top} \quad \text { e } \nabla g\left(x_{1}, x_{2}\right)=\left(\exp x_{2}, x_{1} \exp x_{2}\right)^{\top} .
$$

Desta forma, o cone $K(x)$ em $x^{*}=(0,0)^{\top}$ é $K\left(x^{*}\right)=\left\{\lambda(1,0)^{\top}+\mu(1,0)^{\top}: \lambda \in \mathbb{R}, \mu \geq 0\right\}=\mathbb{R} \times\{0\}$. A condição CAKKT regular vale em $x^{*}$.

Seja $\omega^{*}=\left(\omega_{1}^{*}, \omega_{2}^{*}\right)^{\top} \in \lim \sup _{(x, r) \rightarrow\left(x^{*}, 0\right)} K_{C}(z, r)$. Da definição de limite exterior, existem sequências $\left\{x^{k}\right\}$ e $\left\{\omega^{k}\right\}$ em $\mathbb{R}^{2}$ e $r^{k} \geq 0$ tais que $x^{k} \rightarrow x^{*}, \omega^{k} \rightarrow \omega^{*}, \omega^{k} \in K_{C}\left(z^{k}, r^{k}\right)$ e $r^{k} \rightarrow 0$. Como $\omega^{k} \in K_{C}\left(z^{k}, r^{k}\right)$, existem sequências $\lambda^{k}$ e $\mu^{k} \geq 0$ satisfazendo as seguintes relações

$$
\omega^{k}=\lambda^{k} \nabla h\left(x^{k}\right)+\mu^{k} \nabla g\left(x^{k}\right)=\lambda^{k}(1,0)^{\top}+\mu^{k}\left(\exp x_{2}^{k}, x_{1}^{k} \exp x_{2}^{k}\right)^{\top}
$$

e

$$
\left|\lambda^{k} h\left(x^{k}\right)\right|+\left|\mu^{k} g\left(x^{k}\right)\right|=\left|\lambda^{k} x_{1}^{k}\right|+\left|\mu^{k} x_{1}^{k} \exp x_{2}^{k}\right| \leq r^{k} .
$$


De 3.2.6) e de 3.2.7) obtemos que $\left|\omega_{2}^{k}=\mu^{k} x_{1}^{k} \exp x_{2}^{k}\right| \leq r^{k}$ e $\omega_{2}^{k} \rightarrow 0$. Assim, $\omega^{*}$ está em $\mathbb{R} \times\{0\}$ e CAKKT vale.

A condição SAKKT regular não é satisfeita em $x^{*}=(0,0)^{\top}$.

Escolha $x_{1}^{k}:=1 / k, x_{2}^{k}:=x_{1}^{k}, \mu^{k}:=\left(x_{1}^{k} \exp x_{2}^{k}\right)^{-1}$ e $\lambda^{k}:=-\mu^{k} \exp x_{2}^{k}$. Já que $g\left(x_{1}^{k}, x_{2}^{k}\right)>0$, temos que

$$
\omega^{k}:=\lambda^{k}(1,0)^{\top}+\mu^{k}\left(\exp x_{2}^{k}, x_{1}^{k} \exp x_{2}^{k}\right)^{\top}=(0,1)^{\top} \in N_{\Omega\left(x^{k}, 0\right)}\left(x^{k}\right) \text { para todo } k \in \mathbb{N} \text {. }
$$

Assim, $\lim _{k \rightarrow \infty} \omega^{k}=(0,1)^{\top} \in \lim \sup N_{\Omega(x, 0)}(x)$ mas $(0,1)^{\top}$ não pertence a $\mathbb{R} \times\{0\}$. Portanto, a condição SAKKT regular falha.

Temos apresentado contra-exemplos que mostram que a condição de qualificação AGP regular é estritamente mais forte que as condições de qualificação SAKKT regular e L-AGP regular, e que a condição de qualificação CAKKT é mais fraca que a condição de qualificação SAKKT.

Os seguintes exemplos provam que as condições de qualificação SAKKT regular e L-AGP regular são independentes e que CAKKT regular não implica L-AGP regular.

Exemplo 3.2.5. (SAKKT regular não implica L-AGP regular).

Considere $x^{*}=(0,0)$ em $\mathbb{R}^{2}$ e as restrições de igualdade e desigualdade definida pelas funções

$$
\begin{aligned}
& h\left(x_{1}, x_{2}\right)=x_{1} ; \\
& g\left(x_{1}, x_{2}\right)=-x_{1}^{2}-x_{1}^{2} x_{2}^{2}-x_{2}^{2} .
\end{aligned}
$$

Das restrições, $x^{*}=(0,0)$ é viável e a restrição de desigualdade é ativa em $x^{*}$. Da forma das derivadas

$$
\nabla h\left(x_{1}, x_{2}\right)=(1,0)^{\top} \quad \text { e } \nabla g\left(x_{1}, x_{2}\right)=\left(-2 x_{1}-2 x_{1} x_{2}^{2},-2 x_{2} x_{1}^{2}-2 x_{2}\right)^{\top} \forall x=\left(x_{1}, x_{2}\right)^{\top} \in \mathbb{R}^{2}
$$

temos que $K\left(x^{*}\right)=\left\{\lambda(1,0)^{\top}+\mu(0,0)^{\top}: \lambda \in \mathbb{R}, \mu \in \mathbb{R}_{+}\right\}=\mathbb{R} \times\{0\}$.

$A$ condição $S A K K T$ regular vale em $x^{*}$.

Temos que provar que a multifunção $N_{\Omega(x, 0)}(x)$ é semicontínua exteriormente em $x^{*}=(0,0)^{\top}$. Seja $\omega^{*}=\left(\omega_{1}^{*}, \omega_{2}^{*}\right)^{\top} \in \lim \sup N_{\Omega(x, 0)}(x)$. Da definição de limite exterior, existem sequências $\left\{x^{k}\right\}$ e $\left\{\omega^{k}\right\}$ em $\mathbb{R}^{2}$ tais que $x^{k} \rightarrow x^{*}, \omega^{k} \rightarrow \omega^{*}$ e $\omega^{k} \in N_{\Omega\left(x^{k}, 0\right)}\left(x^{k}\right)$. Analisaremos os diversos casos possíveis. Se existem infinitos índices $k$ tais que:

- O ponto $x_{1}^{k}$ é diferente de zero. Nesse caso $g\left(x_{1}^{k}, x_{2}^{k}\right)=-\left(x_{1}^{k}\right)^{2}\left(1+\left(x_{2}^{k}\right)^{2}\right)-x_{2}^{2}$ é sempre negativo. Assim, o multiplicador associado a $\nabla g\left(x_{1}^{k}, x_{2}^{k}\right)$ é zero (Proposição 3.1.8) portanto $\omega^{k}=\lambda^{k} \nabla h\left(x_{1}^{k}, x_{2}^{k}\right)=\lambda^{k}(1,0)^{\top} \in \mathbb{R} \times\{0\}$ para algum $\lambda^{k} \in \mathbb{R}$. Tomando limites adequados temos que $\omega^{*} \in \mathbb{R} \times\{0\}$;

- O ponto $x_{1}^{k}$ é igual a zero. Nesse caso, $g\left(x_{1}^{k}, x_{2}^{k}\right)=-x_{2}^{2}$. Dependendo de $x_{2}^{k}$ temos os seguintes sub-casos:

- Se $x_{2}^{k}$ é diferente de zero. Assim, $g\left(x_{1}^{k}, x_{2}^{k}\right)<0$. Da Proposição (3.1.8), o multiplicador associado a $\nabla g\left(x^{k}, y^{k}\right)$ é zero. Portanto $\omega^{k}=\lambda^{k} \nabla h\left(x_{1}^{k}, x_{2}^{k}\right)=\lambda^{k}(1,0) \uparrow \in \mathbb{R} \times\{0\}$ para algum $\lambda^{k} \in \mathbb{R}$. Tomando limites adequados temos que $\omega^{*} \in \mathbb{R} \times\{0\}$;

- Se $x_{2}^{k}$ é igual a zero. Nesse sub-caso $g\left(x_{1}^{k}, x_{2}^{k}\right)=(0,0)$, logo existem $\lambda^{k} \in \mathbb{R}$ e $\mu \in \mathbb{R}_{+}$tais que $\omega^{k}=\lambda^{k}(1,0)^{\top}+\mu^{k}\left(-2 x_{1}^{k}-2 x_{1}^{k}\left(x_{2}^{k}\right)^{2},-2 x_{2}^{k}\left(x_{1}^{k}\right)^{2}-2 x_{2}^{k}\right)^{\top}=\lambda^{k}(1,0)^{\top} \in \mathbb{R} \times\{0\}$, onde na última igualdade usamos que $x_{1}^{k}=0$ e $x_{2}^{k}=0$. Logo, $\omega^{k} \in \mathbb{R} \times\{0\}$, e como consequência $\omega^{*} \in \mathbb{R} \times\{0\}$.

Analisando todos os casos concluímos que $\omega^{*}$ pertence a $\mathbb{R} \times\{0\}$. Portanto, a multifunção $N_{\Omega(z, 0)}(z)$ é semicontínua exteriormente em $x^{*}=(0,0)^{\top}$. 
A condição de qualificação L-AGP regular não é satisfeita em $x^{*}=(0,0)^{\top}$.

Calculemos o conjunto das restrições lineares $\Omega_{L}$. Como a única restrição linear é dada pela função $h$ (restrição de igualdade) temos que

$$
\Omega_{L}=\left\{x=\left(x_{1}, x_{2}\right)^{\top} \in \mathbb{R}^{2}: h\left(x_{1}, x_{2}\right)=0\right\}=\left\{x=\left(x_{1}, x_{2}\right)^{\top} \in \mathbb{R}^{2}: x_{1}=0\right\}=\{0\} \times \mathbb{R} .
$$

Defina $x_{1}^{k}:=0, x_{2}^{k}:=1 / k, \varepsilon_{1}^{k}=0$ e $\varepsilon_{2}^{k}=-x_{2}^{k} / 2$. Claramente, essas sequências convergem para zero. Das escolhas temos que $x^{k}$ e $x^{k}+\varepsilon^{k}$ pertence a $\Omega_{L}$. Além disso, temos que $g\left(x_{1}^{k}, x_{2}^{k}\right)=-\left(x_{2}^{k}\right)^{2}$ é negativo e a seguinte relação vale para todo $k \in \mathbb{N}$

$$
g\left(x^{k}, y^{k}\right)+\left\langle\nabla g\left(x^{k}, y^{k}\right),\left(\varepsilon_{1}^{k}, \varepsilon_{2}^{k}\right)^{\top}\right\rangle=0 .
$$

Deste modo podemos escolher $\mu^{k}:=\left(2 x_{2}^{k}\right)^{-1}$ e $\lambda^{k}:=1$ e definir

$\omega^{k}:=\lambda^{k} \nabla h\left(x_{1}^{k}, x_{2}^{k}\right)+\mu^{k} \nabla g\left(x_{1}^{k}, x_{2}^{k}\right)=\lambda^{k}(1,0)^{\top}+\mu^{k}\left(0,-2 x_{2}^{k}\right)^{\top}=(1,-1)^{\top} \in N_{\Omega_{N L}\left(x^{k},-\infty\right) \cap \Omega_{L}}\left(x^{k}+\varepsilon^{k}\right)$.

Assim, $\lim \omega^{k}=(1,-1)^{\top} \in \lim \sup N_{\Omega_{N L}\left(x^{k},-\infty\right) \cap \Omega_{L}}\left(x^{k}+\varepsilon^{k}\right)$ relativo a $\Omega_{L} \times \mathbb{R}^{2} \operatorname{mas}(1,-1)^{\top}$ não pertence a $K\left(x^{*}\right)=\mathbb{R} \times\{0\}$. Assim, L-AGP regular falha em $x^{*}$.

Exemplo 3.2.6. (L-AGP regular não implica SAKKT regular).

Em $\mathbb{R}^{2}$ considere $x^{*}=(0,0)^{\top}$ e as restrições de igualdade e desigualdade definidas pelas funções

$$
\begin{aligned}
& h\left(x_{1}, x_{2}\right)=x_{1} ; \\
& g\left(x_{1}, x_{2}\right)=x_{1}-x_{1}^{2} x_{2} .
\end{aligned}
$$

O exemplo considerado é o exemplo 3.2.3 do qual temos que $K\left(x^{*}\right)=\mathbb{R} \times\{0\}$ e a condição L-AGP regular vale em $x^{*}=(0,0)^{\top}$.

A condição de qualificação SAKKT regular não é satisfeita em $x^{*}=(0,0)^{\top}$.

Escolha $x_{1}^{k}:=1 / k$ e $x_{2}^{k}:=x_{1}^{k}$. Com essa escolha $g\left(x_{1}^{k}, x_{2}^{k}\right)=x_{1}^{k}\left(1-x_{1}^{k} x_{2}^{k}\right)$ é estritamente positivo (para $k$ suficientemente grande) logo podemos escolher $\mu^{k}:=1 /\left(x_{1}^{k}\right)^{2} \in \mathbb{R}_{+}$e $\lambda^{k}=-\mu^{k}\left(1-2 x_{1}^{k} x_{2}^{k}\right)$. Da Proposição 3.1.8, temos que para todo $k \in \mathbb{N}$ :

$\omega^{k}:=\lambda^{k} \nabla h\left(x_{1}^{k}, x_{2}^{k}\right)+\mu^{k} \nabla g\left(x_{1}^{k}, x_{2}^{k}\right)=\lambda^{k}(1,0)^{\top}+\mu^{k}\left(1-2 x_{1}^{k} x_{2}^{k},-\left(x_{1}^{k}\right)^{2}\right)^{\top}=(0,-1)^{\top} \in N_{\Omega\left(x^{k}, 0\right)}\left(x^{k}\right)$.

Assim, temos que $\lim _{k \rightarrow \infty} \omega^{k}=(0,-1)^{\top} \in \lim \sup N_{\Omega\left(x^{k}, 0\right)}\left(x^{k}\right)$. Mas o vetor $(0,-1)^{\top}$ não pertence a $K\left(z^{*}\right)=\mathbb{R} \times\{0\}$, e portanto a condição SAKKT regular não vale em $x^{*}=(0,0)^{\top}$.

Exemplo 3.2.7. (A condição CAKKT regular não implica $L$-AGP regular).

Em $\mathbb{R}^{2}$ considere $x^{*}=(0,0)^{\top}$ e a restrições de desigualdade definidas pelas funções

$$
\begin{aligned}
& g_{1}\left(x_{1}, x_{2}\right)=-x_{1} ; \\
& g_{2}\left(x_{1}, x_{2}\right)=x_{1} \exp x_{2} .
\end{aligned}
$$

Das restrições temos que $x^{*}=(0,0)^{\top}$ é viável e ambas restrições são ativas em $x^{*}$. Calculando as derivadas:

$$
\nabla g_{1}\left(x_{1}, x_{2}\right)=(-1,0)^{\top} \quad \text { e } \quad \nabla g_{2}\left(x_{1}, x_{2}\right)=\left(\exp x_{2}, x_{1} \exp x_{2}\right)^{\top} \text { para todo } x=\left(x_{1}, x_{2}\right)^{\top} \in \mathbb{R}^{2} .
$$

Desta forma, o cone $K\left(x^{*}\right)$ é o conjunto $\left\{\mu_{1}(-1,0)^{\top}+\mu_{2}(1,0)^{\top}: \mu_{1}^{k}, \mu_{2}^{k} \geq 0\right\}=\mathbb{R} \times\{0\}$.

$A$ condição CAKKT regular vale em $x^{*}=(0,0)$.

Seja $\omega^{*}=\left(\omega_{1}^{*}, \omega_{2}^{*}\right)^{\top} \in \lim \sup _{(x, r) \rightarrow\left(x^{*}, 0\right)} K_{C}(x, r)$. Logo existem sequências $\left\{x^{k}\right\}$ e $\left\{\omega^{k}\right\}$ em $\mathbb{R}^{2}$ e $r^{k} \geq 0$ tais que $x^{k} \rightarrow x^{*}, \omega^{k} \rightarrow \omega^{*}, \omega^{k} \in K_{C}\left(x^{k}, r^{k}\right)$ e $r^{k} \rightarrow 0$. Como $\omega^{k} \in K_{C}\left(x^{k}, r^{k}\right)$ existem sequências não negativas $\mu_{1}^{k}$ e $\mu_{2}^{k}$ satisfazendo as relações

$$
\omega^{k}=\mu_{1}^{k} \nabla g_{1}\left(x^{k}\right)+\mu_{2}^{k} \nabla g_{2}\left(x^{k}\right)=\mu_{1}^{k}(-1,0)^{\top}+\mu_{2}^{k}\left(\exp x_{2}, x_{1} \exp x_{2}\right)^{\top}
$$


$\mathrm{e}$

$$
\left|\mu_{1}^{k} g_{1}\left(z^{k}\right)\right|+\left|\mu_{2}^{k} g_{2}\left(z^{k}\right)\right|=\left|\mu_{1}^{k} x_{1}^{k}\right|+\left|\mu_{2}^{k} x_{1}^{k} \exp x_{2}^{k}\right| \leq r^{k} .
$$

De 3.2.9) e (3.2.10), obtemos que $\left|\omega_{2}^{k}=\mu_{2}^{k} x_{1}^{k} \exp x_{2}^{k}\right| \leq r^{k}$ e $\omega_{2}^{k} \rightarrow 0$. Por conseguinte, $\omega^{*} \in \mathbb{R} \times\{0\}$ e CAKKT regular vale.

A condição L-AGP regular não é satisfeita em $x^{*}=(0,0)^{\top}$.

Escolha $x_{1}^{k}:=1 / k, x_{2}^{k}:=x_{1}^{k}, \varepsilon_{1}^{k}:=x_{1}^{k} x_{2}^{k}$ e $\varepsilon_{2}^{k}:=-x_{2}^{k}$. Das escolhas temos que $\varepsilon^{k} \rightarrow(0,0)$. Como $\Omega_{L}=\left\{x=\left(x_{1}, x_{2}\right)^{\top}: g_{1}\left(x_{1}, x_{2}\right)=-x_{1} \leq 0\right\}=\mathbb{R}_{+} \times \mathbb{R}$, as sequências $x^{k}$ e $x^{k}+\epsilon^{k}$ pertencem a $\Omega_{L}$. Como $x^{k}>0$, a restrição não linear $g_{2}\left(x_{1}^{k}, x_{2}^{k}\right)$ é estritamente positiva para todo $k \in \mathbb{N}$ e

$$
\begin{aligned}
\left\langle\nabla g_{2}\left(x_{1}^{k}, x_{2}^{k}\right),\left(\varepsilon_{1}^{k}, \varepsilon_{2}^{k}\right)^{\top}\right\rangle & =\varepsilon_{1}^{k} \exp x_{2}^{k}+\varepsilon_{2}^{k} x_{1}^{k} \exp x_{2}^{k} \\
& =x_{1}^{k} x_{2}^{k} \exp x_{2}^{k}+\left(-x_{2}^{k}\right) x_{1}^{k} \exp x_{2}^{k}=0 .
\end{aligned}
$$

Assim, podemos escolher livremente o multiplicador associado a $\nabla g_{2}\left(x_{1}^{k}, x_{2}^{k}\right)$. Escolha os multiplicadores $\mu_{2}^{k}:=\left(x_{1}^{k} \exp x_{2}^{k}\right)^{-1}$ e $\mu_{1}^{k}:=\mu_{2}^{k} \exp x_{2}^{k}$. Defina $\omega^{k}:=\mu_{1}^{k}(-1,0)^{\top}+\mu_{2}^{k}\left(\exp x_{2}^{k}, x_{1}^{k} \exp x_{2}^{k}\right)^{\top}=$ $(0,1)^{\top}$. Das escolhas dos multiplicadores, temos que $\omega^{k}=(0,1)^{\top} \in N_{\Omega_{N L}\left(x^{k},-\infty\right) \cap \Omega_{L}}\left(x^{k}+\varepsilon^{k}\right)$ para todo $k \in \mathbb{N}$. Como consequência dessa inclusão, $(0,1)^{\top} \in \lim \sup _{(x, \varepsilon) \rightarrow\left(x^{*}, 0\right), x \in \Omega_{L}} N_{\Omega_{N L}(x,-\infty) \cap \Omega_{L}}(x+$ $\varepsilon)$ mas $(0,1)^{\top}$ não pertence a $\mathbb{R} \times\{0\}$, o que mostra que a condição L-AGP regular não vale em $x^{*}$.

A Figura 3.6 mostra as relações entre as novas condições de qualificação introduzidas nesta seção.

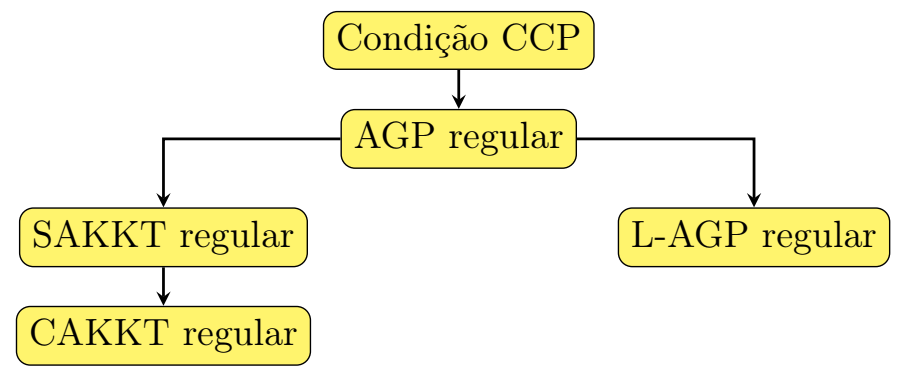

Figura 3.6: Relações entre as novas condições de qualificação

\subsection{Relações com as condições de qualificação conhecidas.}

Sempre que se definem novas condições de qualificação, é natural perguntarmos qual é a exata relação com as condições de qualificação já estabelecidas na literatura. Nesta seção nos focamos em mostrar essas relações existentes.

\subsubsection{Relação com a condição de qualificação CPG.}

Nesta subseção mostraremos que a Propriedade de Continuidade do Cone é estritamente mais fraca que a $\mathrm{CPG}$

Teorema 3.3.1. A condição CPG implica a condição CCP.

Demonstração. Da definição de CPG, existem subconjuntos de índices $I^{\prime}, J^{\prime}$ e $J_{+}$tais que os gradientes $\nabla h_{i}\left(x^{*}\right)$ e $\nabla g_{j}\left(x^{*}\right)$ com $(i, j) \in\left(I^{\prime}, J^{\prime}\right)$ são linearmente independentes em uma vizinhança $V$ de $x^{*}$ tal que

$$
K(x) \subset K_{I^{\prime}, J^{\prime}}(x) \quad \forall x \in V .
$$

Tomando limites em (3.3.1) quando $x \rightarrow x^{*}$ e usando a semicontinuidade externa de $K_{I^{\prime}, J^{\prime}}(x)$ em $x^{*}$, ver Lema(2.3.1), temos que

$$
\limsup _{x \rightarrow x^{*}} K(x) \subset \limsup _{x \rightarrow x^{*}} K_{I^{\prime}, J^{\prime}}(x) \subset K_{I^{\prime}, J^{\prime}}\left(x^{*}\right)=K\left(x^{*}\right) .
$$


Por conseguinte, CCP vale em $x^{*}$.

O próximo exemplo mostra que, de fato, a condição CCP é estritamente mais fraca que CPG.

Exemplo 3.3.1. (CCP não implica $C P G$.)

Em $\mathbb{R}^{2}$, consideremos $x^{*}=(0,0)^{\top}$ e restrições de desigualdades definidas por

$$
\begin{aligned}
& g_{1}\left(x_{1}, x_{2}\right)=x_{1} ; \\
& g_{2}\left(x_{1}, x_{2}\right)=\left(x_{1}^{+}\right)^{2} \exp \left(\left(x_{2}^{+}\right)^{2}\right),
\end{aligned}
$$

onde $a^{+}:=\max \{0, a\}$, isto é, a parte positiva de $a$. Claramente, $x^{*}=(0,0)^{\top}$ é um ponto viável e ambas restrições são ativas em $x^{*}$. Temos também que

$\nabla g_{1}\left(x_{1}, x_{2}\right)=(1,0)^{\top}$ e $\nabla g_{2}\left(x_{1}, x_{2}\right)=\left(2 x_{1}^{+} \exp \left(\left(x_{2}^{+}\right)^{2}\right), 2 x_{2}^{+}\left(x_{1}^{+}\right)^{2} \exp \left(\left(x_{2}^{+}\right)^{2}\right)\right)^{\top}$ para $x=\left(x_{1}, x_{2}\right)^{\top} \in \mathbb{R}^{2}$.

De $\nabla g_{1}\left(x^{*}\right)=(1,0)^{\top}$ e de $\nabla g_{2}\left(x^{*}\right)=(0,0)^{\top}$, temos que $K\left(x^{*}\right)=\mathbb{R}_{+} \times\{0\}$ e a única possível escolha para uma base positiva de $K\left(x^{*}\right)$ é $\left\{I^{\prime}=\emptyset, J^{\prime}=\emptyset, J_{+}=\{1\}\right\}$. Assim, $K_{I^{\prime}, J^{\prime}}(x)=\mathbb{R}_{+} \times\{0\}$ para qualquer $x=\left(x_{1}, x_{2}\right)^{\top} \in \mathbb{R}^{2}$. Por outro lado

$$
K(x)=\left\{\left(\mu_{1}+2 \mu_{2} x_{1}^{+} \exp \left(\left(x_{2}^{+}\right)^{2}\right), 2 \mu_{2} x_{2}^{+}\left(x_{1}^{+}\right)^{2} \exp \left(\left(x_{2}^{+}\right)^{2}\right)\right)^{\top} \mid \quad \mu_{1}, \mu_{2} \geq 0\right\} .
$$

Claramente $K(x)$ não pode ser um subconjunto de $K_{I^{\prime}, J^{\prime}}(x)$ em nenhuma vizinhança de $x^{*}=(0,0)$ (escolha quaisquer $x_{1}>0, x_{2}>0$ próximos a zero e $\mu_{2}>0$ ) e como consequência CPG falha.

Agora procederemos a provar que $K(x)$ é semicontínua exterior em $x^{*}$. Seja $\omega^{*} \in \lim \sup _{x \rightarrow x^{*}} K(x)$. Por conseguinte, existem sequências $\left\{x^{k}\right\}$ e $\left\{\omega^{k}\right\}$ tais que $x^{k}=\left(x_{1}^{k}, x_{2}^{k}\right)^{\top} \rightarrow x^{*}, \omega^{k}=\left(\omega_{1}^{k}, \omega_{2}^{k}\right) \rightarrow \omega^{*}$ e

$$
\omega^{k}=\mu_{1}^{k}(1,0)^{\top}+\mu_{2}^{k}\left(2 x_{1}^{+} \exp \left(\left(x_{2}^{+}\right)^{2}\right), 2 x_{2}^{+}\left(x_{1}^{+}\right)^{2} \exp \left(\left(x_{2}^{+}\right)^{2}\right)\right)^{\top} \in K\left(x^{k}\right),
$$

para certos $\mu_{1}^{k}, \mu_{2}^{k} \geq 0$. Agora, suponha por contradição que $\omega^{*}=\left(\omega_{1}^{*}, \omega_{2}^{*}\right)^{\top}$ não pertence ao cone $K\left(x^{*}\right)=\mathbb{R}_{+} \times\{0\}$. Assim, $\omega_{2}^{*}$ deve ser diferente de zero. De 3.3.3) temos que existe $\rho>0$ tal que

$$
\left.\left|\omega_{2}^{k}\right|=2 \mu_{2}^{k} \mid\left(x_{2}^{k}\right)^{+}\left(\left(x_{2}^{k}\right)+\right)^{2} \exp \left(x_{2}^{k}\right)^{+}\right)^{2} \mid>\rho>0,
$$

para $k$ suficientemente grande. Usando $\mu_{1}^{k} \geq 0$ e 3.3.4, temos

$$
\omega_{1}^{k}=\mu_{1}^{k}+2 \mu_{2}^{k}\left(x_{1}^{k}\right)^{+} \exp \left(\left(x_{2}^{k}\right)^{+}\right)^{2} \geq \frac{\left|\omega_{2}^{k}\right|}{\left(x_{1}^{k}\right)^{+}\left(x_{2}^{k}\right)^{+}}>\frac{\rho}{\left(x_{1}^{k}\right)^{+}\left(x_{2}^{k}\right)^{+}}>0 .
$$

Tomando limites em (3.3.5) chegamos a uma contradição. Assim, $\omega^{*}$ pertence a $K\left(x^{*}\right)$.

Neste exemplo, o cone $K(x)$ é o cone positivo gerado por $(1,0)^{\top}$ e por $\left(1, x_{1}^{+} x_{2}^{+}\right)^{\top}$.

\subsubsection{Relação com a condição de qualificação de Abadie.}

Nesta subseção mostraremos que a condição de qualificação de Abadie é estritamente mais fraca que as condições de qualificação CAKKT regular e L-AGP regular. As provas estão baseadas nos seguintes lemas:

Lema 3.3.2. (RW09, Teorema 6.11) Seja $\bar{x} \in \Omega$. Para qualquer $y \in T_{\Omega}^{\circ}(\bar{x})$, existe uma função $F$ derivável e com derivada contínua tal que $-\nabla F(\bar{x})=y$. Ainda mais, $F$ atinge o mínimo global estrito sobre $\Omega$ em $\bar{x}$.

Lema 3.3.3. Para $y \in T_{\Omega}^{\circ}(\bar{x})$, existem sequências $\left\{x^{k}\right\} \subset \mathbb{R}^{n},\left\{\lambda^{k}\right\} \subset \mathbb{R}^{m},\left\{\mu^{k}\right\} \subset \mathbb{R}_{+}^{p}$ tais que:

1. A sequência $\left\{x^{k}\right\}$ converge para $\bar{x}$;

2. $\omega^{k}:=\sum_{i=1}^{m} \lambda_{i}^{k} \nabla h_{i}\left(x^{k}\right)+\sum_{j=1}^{p} \mu_{j}^{k} \nabla g_{j}\left(x^{k}\right) \rightarrow y$; 
3. $\mu_{j}^{k}$ é proporcional a $\max \left\{0, g_{j}\left(x^{k}\right)\right\} \forall j \in\{1, . ., p\}$;

4. $r^{k}:=\sum_{i=1}^{m}\left|\lambda_{i}^{k} h_{i}\left(x^{k}\right)\right|+\sum_{j=1}^{p}\left|\mu_{j}^{k} g_{j}\left(x^{k}\right)\right| \rightarrow 0$.

Demonstração. Seja $y \in T_{\Omega}^{\circ}(\bar{x})$. Do Lema 3.3.2, existe uma função $F$ derivável e com derivada contínua tal que $-\nabla F(\bar{x})=y$ e $F$ atinge o mínimo global estrito sobre $\Omega$ em $\bar{x}$. Seja $r>0$ fixo, e para cada $k \in \mathbb{N}$, considere o seguinte problema de otimização

$$
\operatorname{minimizar} \quad F_{k}(x):=F(x)+\frac{k}{2}\left(\sum_{j=1}^{p} \max \left\{g_{j}(x), 0\right\}^{2}+\sum_{i=1}^{m} h_{i}^{2}(x)\right)
$$

sujeito a $x \in \mathbb{B}(\bar{x}, r)$.

Devido ao Teorema de Weierstrass, existe uma solução $x^{k}$ para o problema penalizado 3.3.6. Seguindo a mesma linha de argumentação do Teorema 2.4.10, temos que $x^{k} \rightarrow \bar{x}$,

$$
\nabla F\left(x^{k}\right)+\sum_{i=1}^{m} k h_{i}\left(x^{k}\right) \nabla h_{i}\left(x^{k}\right)+\sum_{j=1}^{p} k \max \left\{g_{j}\left(x^{k}\right), 0\right\} \nabla g_{j}\left(x^{k}\right)=0
$$

e

$$
\sum_{i=1}^{m} k h_{i}\left(x^{k}\right)^{2}+\sum_{j=1}^{p} k \max \left\{g_{j}\left(x^{k}\right), 0\right\}^{2} \leq F(\bar{x})-F\left(x^{k}\right) .
$$

Defina $\lambda_{i}^{k}:=k h_{i}\left(x^{k}\right)$ para todo $i \in\{1, \ldots, m\}$ e $\mu_{j}^{k}:=k \max \left\{g_{j}\left(x^{k}\right) 0\right\}$ para todo $j \in\{1, \ldots, p\}$. Agora, defina $\omega^{k}:=\sum_{j=1}^{p} k \max \left\{g_{j}\left(x^{k}\right), 0\right\} \nabla g_{j}\left(x^{k}\right)+\sum_{i=1}^{m} k h_{i}\left(x^{k}\right) \nabla h_{i}\left(x^{k}\right)$. Da expressão 3.3.7 temos que $\omega^{k} \rightarrow y$. Agora, defina $r^{k}:=\sum_{i=1}^{m}\left|\lambda_{i}^{k} h_{i}\left(x^{k}\right)\right|+\sum_{j=1}^{p}\left|\mu_{j}^{k} g_{j}\left(x^{k}\right)\right|$. Como $F$ é limitado em $\mathbb{B}(\bar{x}, r)$ temos que 3.3 .8 implica $r^{k} \rightarrow 0$.

Lema 3.3.4. Para $y \in T_{\Omega}^{\circ}(\bar{x})$, existem sequências $\left\{x^{k}\right\} \subset \Omega_{L},\left\{\omega^{k}\right\} \subset \mathbb{R}^{m}$ tais que $x^{k} \rightarrow \bar{x}$, $\omega^{k} \rightarrow y$ $e \omega^{k} \in N_{\Omega_{N L}\left(x^{k},-\infty\right) \cap \Omega_{L}}\left(x^{k}\right)$.

Demonstração. Seja $y \in T_{\Omega}^{\circ}(\bar{x})$. Do Lema 3.3.2, existe $F$ tal que $-\nabla F(\bar{x})=y$ e $F$ atinge o mínimo global estrito sobre $\Omega$ em $\bar{x}$. Sejam $\left\{g_{j}: j \in\left\{1, . ., p_{1}\right\}\right\}$ e $\left\{h_{i}: i \in\left\{1, . ., m_{1}\right\}\right\}$ as restrições de desigualdade e de igualdade não lineares.

Escolha $r>0$. Considere, para cada $k \in \mathbb{N}$, o problema de otimização

$$
\operatorname{minimizar} \quad F_{k}(x):=F(x)+\frac{k}{2}\left(\sum_{j=1}^{p_{1}} \max \left\{g_{j}(x), 0\right\}^{2}+\sum_{i=1}^{m_{1}} h_{i}^{2}(x)\right)
$$

sujeito a $x \in \mathbb{B}(\bar{x}, r) \cap \Omega_{L}$.

Do Teorema de Weierstrass, existe uma solução $x^{k}$ para o problema penalizado (3.3.9). Seguindo a mesma linha de argumentação do Teorema 2.4.10, temos que a sequência $\left\{x^{k}\right\}$ converge para $\bar{x}$. Para $k$ suficientemente grande, $x^{k} \in \operatorname{Int}(\mathbb{B}(\bar{x}, r))$. Usando a condição geométrica de otimalidade temos que $\left\langle\nabla F_{k}\left(x^{k}\right), d\right\rangle \geq 0$ para todo $d \in T_{\Omega_{L}}\left(x^{k}\right)$ ou equivalentemente

$$
-\nabla F_{k}\left(x^{k}\right) \in N_{\Omega_{L}}\left(x^{k}\right)=T_{\Omega_{L}}^{\circ}\left(x^{k}\right)
$$

Derivando $F_{k}$ temos que

$$
-\left(\nabla F\left(x^{k}\right)+\sum_{j=1}^{p_{1}} k \max \left\{g_{j}\left(x^{k}\right), 0\right\} \nabla g_{j}\left(x^{k}\right)+\sum_{i=1}^{n_{1}} k h_{i}\left(x^{k}\right) \nabla h_{i}\left(x^{k}\right)\right) \in N_{\Omega_{L}}\left(x^{k}\right) .
$$

Defina $\lambda_{i}^{k}:=k h_{i}\left(x^{k}\right)$ para $i \in\left\{1, \ldots, m_{1}\right\}$ e $\mu_{j}^{k}:=k \max \left\{g_{j}\left(x^{k}\right), 0\right\}$ para $j \in\left\{1, \ldots, p_{1}\right\}$. Com essas escolhas, defina $\omega_{1}^{k}:=\sum_{j=1}^{p_{1}} \mu_{j}^{k} \nabla g_{j}\left(x^{k}\right)+\sum_{i=1}^{m_{1}} \lambda_{i}^{k} \nabla h_{i}\left(x^{k}\right)$ e $\omega_{2}^{k}:=-\nabla F\left(x^{k}\right)-\omega_{1}^{k}$. Usando 
a definição do cone $\Omega_{N L}\left(x^{k},-\infty\right)$ e 3.3 .10 temos que $\omega_{1}^{k} \in N_{\Omega_{N L}\left(x^{k},-\infty\right)}\left(x^{k}\right)$ e $\omega_{2}^{k} \in N_{\Omega_{L}}\left(x^{k}\right)$. Defina $\omega^{k}:=-\omega_{1}^{k}+\omega_{2}^{k}=-\nabla F\left(x^{k}\right)$. Claramente, $\omega^{k} \rightarrow-\nabla F\left(x^{*}\right)=y \mathrm{e}$

$$
\omega^{k}=\omega_{1}^{k}+\omega_{2}^{k} \in N_{\Omega_{N L}\left(x^{k},-\infty\right)}\left(x^{k}\right)+N_{\Omega_{L}}\left(x^{k}\right) \subset N_{\Omega_{N L}\left(x^{k},-\infty\right) \cap \Omega_{L}}\left(x^{k}\right) .
$$

A sequência $\left\{\omega^{k}\right\}$ cumpre com as propriedades desejadas.

O seguinte teorema mostra que a condição CAKKT regular implica a condição de qualificação de Abadie.

Teorema 3.3.5. Seja $x^{*} \in \Omega$. Se $x^{*}$ é um ponto CAKKT regular então temos que $x^{*}$ satisfaz a condição de qualificação de Abadie.

Demonstração. Nosso objetivo é provar que $T_{\Omega}\left(x^{*}\right)=L\left(x^{*}\right)$. A inclusão $T_{\Omega}\left(x^{*}\right) \subset L\left(x^{*}\right)$ sempre é válida, independentemente de qualquer condição de qualificação, ver Proposição 2.1.4. Para mostrar a outra inclusão $L\left(x^{*}\right) \subset T_{\Omega}\left(x^{*}\right)$, primeiro provaremos que $N_{\Omega}\left(x^{*}\right) \subset L_{\Omega}^{\circ}\left(x^{*}\right)$, ou equivalentemente $N_{\Omega}\left(x^{*}\right) \subset K_{C}\left(x^{*}, 0\right)$ (Pelo lema de Farkas, $L_{\Omega}^{\circ}\left(x^{*}\right)=K\left(x^{*}\right)$ e para $x^{*} \in \Omega, K_{C}\left(x^{*}, 0\right)=K\left(x^{*}\right)$ ).

Seja $y \in N_{\Omega}\left(x^{*}\right)$, da definição do cone normal 2.1.7, existem sequências $\left\{x^{k}\right\} \subset \Omega,\left\{y^{k}\right\} \subset \mathbb{R}^{n}$ tais que

$$
x^{k} \rightarrow x^{*} \quad, \quad y^{k} \rightarrow y \quad \text { e } y^{k} \in T_{\Omega}^{\circ}\left(x^{k}\right) .
$$

Usando o Lema 3.3 .3 para cada $y^{k} \in T_{\Omega}^{\circ}\left(x^{k}\right)$ encontramos sequências com limites $x^{k}$ e $y^{k}$ tais que eles satisfazem os itens do Lema 3.3.3. Assim para cada $k \in \mathbb{N}$, existem $j(k) \in \mathbb{N}$, escalares $r^{j(k)}$ e vetores $x^{j(k)}$ e $\omega^{j(k)}$ tais que

- $\left\|x^{k}-x^{j(k)}\right\|<1 / 2^{k}$ para todo $k \in \mathbb{N}$;

- $\omega^{j(k)}=\sum_{i=1}^{m} \lambda_{i}^{j(k)} \nabla h_{i}\left(x^{j(k)}\right)+\sum_{s=1}^{p} \mu_{s}^{j(k)} \nabla g_{s}\left(x^{j(k)}\right)$;

- $\left\|y^{k}-w^{j(k)}\right\|<1 / 2^{k}$ para todo $k \in \mathbb{N}$;

- $\mu_{s}^{j(k)}=j(k) \max \left\{g_{s}\left(x^{j(k)}\right), 0\right\}$ para todo $s \in\{1, . ., p\}$.

- $r^{j(k)}=\sum_{i=1}^{m}\left|\lambda_{i}^{j(k)} h_{i}\left(x^{j(k)}\right)\right|+\sum_{s=1}^{p}\left|\mu_{s}^{j(k)} g_{s}\left(x^{j(k)}\right)\right| \leq 1 / 2^{k}$ para todo $k \in \mathbb{N}$.

Claramente, as sequências $\left\{r^{j(k)}\right\},\left\{x^{j(k)}\right\}$ e $\left\{\omega^{j(k)}\right\}$ convergem para $0, x^{*}$ e $y$ respectivamente. Ainda mais, para $k$ suficientemente grande, $\omega^{j(k)}$ pertence a $K_{C}\left(x^{j(k)}, r^{j(k)}\right)$, já que para $k$ suficientemente grande, $\mu_{s}^{j(k)}=j(k) \max \left\{g_{s}\left(x^{j(k)}\right), 0\right\}=0$ para $s \notin J\left(x^{*}\right)$. Assim, as sequências cumprem que $x^{j(k)} \rightarrow x^{*}, \omega^{j(k)} \rightarrow y, r^{j(k)} \rightarrow 0$ e $\omega^{j(k)} \in K_{C}\left(x^{j(k)}, r^{j(k)}\right)$. Da definição de limite exterior temos que $y \in \lim \sup _{(x, r) \rightarrow\left(x^{*}, 0\right)} K_{C}(x, r) \subset K\left(x^{*}\right)$. A última inclusão vem do fato que a condição CAKKT vale em $x^{*}$. Assim, $N_{\Omega}\left(x^{*}\right) \subset K\left(x^{*}\right)=L_{\Omega}\left(x^{*}\right)^{\circ}$ implica

$$
L_{\Omega}\left(x^{*}\right)=K^{\circ}\left(x^{*}\right) \subset N_{\Omega}^{\circ}\left(x^{*}\right) \subset T_{\Omega}\left(x^{*}\right),
$$

onde a relação $N_{\Omega}^{\circ}\left(x^{*}\right) \subset T_{\Omega}\left(x^{*}\right)$ se justifica pelo Teorema 2.1.1 (b).

Analogamente, para a condição de qualificação L-AGP regular temos o seguinte teorema.

Teorema 3.3.6. Seja $x^{*} \in \Omega$ um ponto $L$-AGP regular. Então, temos que $x^{*}$ satisfaz a condição de qualificação de Abadie.

Demonstração. Basta mostrar que $N_{\Omega}\left(x^{*}\right) \subset L_{\Omega}^{\circ}\left(x^{*}\right)$. Seja $y \in N_{\Omega}\left(x^{*}\right)$, da definição de cone normal existem sequências $\left\{x^{k}\right\} \subset \Omega,\left\{y^{k}\right\} \subset \mathbb{R}^{n}$ tais que

$$
x^{k} \rightarrow x^{*} \quad, y^{k} \rightarrow y \quad \text { e } y^{k} \in T_{\Omega}^{\circ}\left(x^{k}\right) .
$$

Usando o Lema 3.3 .4 para $y^{k} \in T_{\Omega}^{\circ}\left(x^{k}\right)$ temos que é possível encontrar sequências que convergem a $x^{k}$ e a $y^{k}$ com as propriedades ali mencionadas, assim existe, para cada $k \in \mathbb{N}$, um número $j(k) \in \mathbb{N}$ e vetores $x^{j(k)}, \omega^{j(k)}$ tais que 
- $\left\|x^{k}-x^{j(k)}\right\|<1 / 2^{k}$ para todo $k \in \mathbb{N}$;

- $\omega^{j(k)} \in N_{\Omega_{N L}\left(x^{j(k)},-\infty\right) \cap \Omega_{L}}\left(x^{j(k)}\right)$;

- $\left\|y^{k}-w^{j(k)}\right\|<1 / 2^{k}$ para todo $k \in \mathbb{N}$;

Logo temos sequências $\left\{x^{j(k)}\right\} \subset \Omega_{L}$ e $\left\{\omega^{j(k)}\right\} \subset N_{\Omega_{N L}\left(x^{j(k)},-\infty\right) \cap \Omega_{L}}\left(x^{j(k)}\right)$ tais que $x^{j(k)} \rightarrow x^{*}$ e $\omega^{j(k)} \rightarrow y$. Portanto $y \in \lim \sup _{(x, \varepsilon) \rightarrow\left(x^{*}, 0\right), x \in \Omega_{L}} N_{\Omega_{N L}(x,-\infty) \cap \Omega_{L}}(x+\varepsilon)$ e como $x^{*}$ é um ponto L-AGP regular, temos que $y \in N_{\Omega_{N L}\left(x^{*},-\infty\right) \cap \Omega_{L}}\left(x^{*}\right)=K\left(x^{*}\right)$ o que nos permite concluir que $N_{\Omega}\left(x^{*}\right) \subset K\left(x^{*}\right)=L\left(x^{*}\right)^{\circ}$. Usando o Teorema 2.1.1 (b), $L\left(x^{*}\right)=K^{\circ}\left(x^{*}\right) \subset N_{\Omega}^{\circ}\left(x^{*}\right) \subset T_{\Omega}\left(x^{*}\right)$, isto é, a condição de qualificação de Abadie vale em $x^{*}$.

O seguinte exemplo mostra que a condição de qualificação de Abadie é estritamente mais fraca que a condição CAKKT regular e a condição L-AGP regular.

Exemplo 3.3.2. (A condição de Abadie não implica CAKKT regular nem L-AGP regular).

Em $\mathbb{R}^{2}$, considere $x^{*}=(0,0)^{\top}$ e as restrições de desigualdades dada pelas funções

$$
\begin{aligned}
& g_{1}\left(x_{1}, x_{2}\right)=-x_{1} ; \\
& g_{2}\left(x_{1}, x_{2}\right)=-x_{2} \exp x_{2} ; \\
& g_{3}\left(x_{1}, x_{2}\right)=-x_{1} x_{2} .
\end{aligned}
$$

Note que todas as desigualdades são ativas em $x^{*}=(0,0)^{\top}$. Calculando os gradientes temos $\nabla g_{1}\left(x_{1}, x_{2}\right)=(-1,0)^{\top}, \quad \nabla g_{2}\left(x_{1}, x_{2}\right)=\left(0,-\exp x_{2}-x_{2} \exp x_{2}\right)^{\top} \quad$ e $\nabla g_{3}\left(x_{1}, x_{2}\right)=\left(-x_{2},-x_{1}\right)^{\top}$, para todo $x=\left(x_{1}, x_{2}\right)^{\top} \in \mathbb{R}^{2}$. Portanto, o cone linearizado é $K\left(x^{*}\right)=\mathbb{R}_{-} \times \mathbb{R}_{-}$.

A condição de Abadie vale em $x^{*}$.

Uma vez que $\Omega=\mathbb{R}_{+}^{2}$ e usando a forma dos gradientes, a condição de Abadie vale em $x^{*}$.

$A$ condição $C A K K T$ regular falha.

Mostraremos que a multifunção $K_{C}(x, r)$ não é semicontínua exteriormente em $\left(x^{*}=(0,0), r=0\right)$. Escolha $x_{1}^{k}:=1 / k, x_{2}^{k}:=-1 / k$ e multiplicadores $\mu_{1}^{k}:=0, \mu_{2}^{k}:=0$ e $\mu_{3}^{k}:=k$. Com essas escolhas temos que

$$
r^{k}:=\left|\mu_{1}^{k} x_{1}^{k}\right|+\left|\mu_{2}^{k} x_{2}^{k} \exp \left(x_{2}^{k}\right)\right|+\left|\mu_{3}^{k} x_{1}^{k} x_{2}^{k}\right|=\frac{k}{k^{2}}=\frac{1}{k} \rightarrow 0
$$

e

$$
\left.\omega^{k}:=\mu_{1}^{k} \nabla g_{1}\left(x_{1}^{k}, x_{2}^{k}\right)+\mu_{2}^{k} \nabla g_{2}\left(x_{1}^{k}, x_{2}^{k}\right)+\mu_{3}^{k} \nabla g_{3}\left(x_{1}^{k}, x_{2}^{k}\right)\right)=k(1 / k,-1 / k)^{\top}=(1,-1)^{\top} .
$$

Como $\omega^{k}=(1,-1)^{\top} \in K_{C}\left(x^{k}, r^{k}\right) \forall k \in \mathbb{N}$, o vetor $(1,-1)^{\top}$ pertence $\operatorname{alim} \sup _{(x, r) \rightarrow\left(x^{*}, 0\right)} K_{C}(x, r)$ mas $(1,-1)^{\top}$ não pertence a $K_{C}\left(\left(z^{*}, 0\right)\right)=\mathbb{R}_{-} \times \mathbb{R}_{-}$. Assim, CAKKT regular não vale em $x^{*}=$ $(0,0)^{\top}$.

A condição $L-A G P$ regular falha.

Note que $\Omega_{L}=\left\{x=\left(x_{1}, x_{2}\right)^{\top}: x_{1} \geq 0\right\}$. Escolha $x_{1}^{k}:=1 / k, x_{2}^{k}:=-1 / k, \varepsilon_{1}^{k}:=0, \varepsilon_{1}^{k}:=0 \mathrm{e}$ multiplicadores $\mu_{1}^{k}:=0, \mu_{2}^{k}:=0$ e $\mu_{3}^{k}:=k$. Com essa escolhas

$$
\left.\omega^{k}:=\mu_{1}^{k} \nabla g_{1}\left(x_{1}^{k}, x_{2}^{k}\right)+\mu_{2}^{k} \nabla g_{2}\left(x_{1}^{k}, x_{2}^{k}\right)+\mu_{3}^{k} \nabla g_{3}\left(x_{1}^{k}, x_{2}^{k}\right)\right) \in N_{\Omega_{L} \cap \Omega\left(x^{k},-\infty\right)}\left(x^{k}+\varepsilon^{k}\right) .
$$

A sequência $x^{k}=(1 / k,-1 / k)^{\top}$ pertence a $\Omega_{L}$ para todo $k \in \mathbb{N}$ e $x^{k} \rightarrow(0,0)^{\top}$. Assim temos que $\lim _{k \rightarrow \infty} \omega^{k}$ pertence a $\lim \sup _{(x, \varepsilon) \rightarrow\left(x^{*}, 0\right), x \in \Omega_{L}} N_{\Omega_{L} \cap \Omega(x,-\infty)}(x+\varepsilon)$. Pela escolha das sequências e multiplicadores, $\omega^{k}=(1,-1)^{\top}$ para todo $k \in \mathbb{N}$. Portanto L-AGP regular falha em $x^{*}$. 


\subsubsection{Relação com a Pseudonormalidade e Quasinormalidade}

Nesta subseção provaremos as relações existentes entre as novas condições de qualificação e a Pseudonormalidade e a Quasinormalidade.

O seguinte exemplo mostra que a Pseudonormalidade não implica a condição CAKKT regular nem a condição L-AGP regular.

Exemplo 3.3.3. (Pseudonormalidade não implica CAKKT regular nem L-AGP regular).

Em $\mathbb{R}^{2}$, considere $x^{*}=(0,0)^{\top}$ e as restrições de igualdade e desigualdades dada pelas funções

$$
\begin{aligned}
& h\left(x_{1}, x_{2}\right)=x_{2}-x_{1} ; \\
& g\left(x_{1}, x_{2}\right)=x_{1}-x_{2} \exp x_{2} .
\end{aligned}
$$

O ponto $x^{*}=(0,0)$ é viável e para ambas restrições de desigualdade são ativas em $x^{*}$. Já os gradientes são:

$$
\nabla h\left(x_{1}, x_{2}\right)=(-1,1)^{\top} \quad \text { e } \nabla g\left(x_{1}, x_{2}\right)=\left(1,-\exp x_{2}-x_{2} \exp x_{2}\right)^{\top} \text { para } x=\left(x_{1}, x_{2}\right)^{\top} \in \mathbb{R}^{2} .
$$

Assim, o cone $K\left(x^{*}\right)=\left\{\lambda(-1,1)^{\top}+\mu(1,-1)^{\top}: \lambda \in \mathbb{R}, \mu \in \mathbb{R}_{+}\right\}=\mathbb{R}(-1,1)^{\top}$ é o subespaço gerado pelo vetor $(-1,1)$.

Pseudonormalidade vale em $x^{*}=(0,0)^{\top}$.

Claramente, $\nabla g\left(x^{*}\right)=-\nabla h\left(x^{*}\right)=(-1,1)^{\top}$. Se $\mu \nabla g\left(x^{*}\right)+\lambda \nabla h\left(x^{*}\right)=(0,0)^{\top}$ para $\mu \in \mathbb{R}_{+}$e $\lambda \in \mathbb{R}$ não nulos, devemos ter que $\mu=\lambda>0$. Suponha, por contradição, que existe uma sequência $\left(x_{1}^{k}, x_{2}^{k}\right) \rightarrow(0,0)^{\top}$, tal que $\lambda h\left(x_{1}^{k}, x_{2}^{k}\right)+\mu g\left(x_{1}^{k}, x_{2}^{k}\right)>0$ para todo $k \in \mathbb{N}$. Assim, $\lambda h\left(x_{1}^{k}, x_{2}^{k}\right)+$ $\mu g\left(x_{1}^{k}, x_{2}^{k}\right)=\mu\left(x_{2}^{k}-x_{1}^{k}+x_{1}^{k}-x_{2}^{k} \exp x_{2}^{k}\right)=\mu\left(x_{2}^{k}-x_{2}^{k} \exp x_{2}^{k}\right)>0$ implica que $x_{2}>x_{2} \exp x_{2}$ para todo $k \in \mathbb{N}$, mas isso é impossível, porque não existe $x_{2} \neq 0$ tal que $x_{2}>x_{2} \exp x_{2}$. Portanto, Pseudonormalidade vale em $x^{*}$.

A condição CAKKT regular não vale em $x^{*}=(0,0)^{\top}$.

Escolha $x_{1}^{k}:=1 / k, x_{2}^{k}:=x_{1}^{k}, \mu^{k}:=-\left(1-\exp x_{2}^{k}-x_{2}^{k} \exp x_{2}^{k}\right)^{-1}$ e $\lambda^{k}:=2-\mu^{k}\left(-\exp x_{2}^{k}-x_{2}^{k} \exp x_{2}^{k}\right)$. Defina

$$
\omega^{k}:=\lambda^{k}(-1,1)^{\top}+\mu^{k}\left(1,-\exp x_{2}^{k}-x_{2}^{k} \exp x_{2}^{k}\right)^{\top} .
$$

Provaremos que $\omega^{k} \rightarrow(-3,2)^{\top}, r^{k}:=\left|\lambda^{k} h\left(x_{1}^{k}, x_{2}^{k}\right)\right|+\left|\mu^{k} g\left(x_{1}^{k}, x_{2}^{k}\right)\right| \rightarrow 0$ e $\omega^{k} \in K_{C}\left(x^{k}, r^{k}\right) \forall k \in \mathbb{N}$. Das escolhas temos que $\mu^{k} \geq 0, \omega_{2}^{k}=\lambda^{k}+\mu^{k}\left(-\exp x_{2}^{k}-x_{2}^{k} \exp x_{2}^{k}\right)=2$ e $\omega_{1}^{k}=-\lambda^{k}+\mu^{k}=$ $-2+\mu^{k}\left(1-\exp x_{2}^{k}-x_{2}^{k} \exp x_{2}^{k}\right)=-3$. Assim, temos que $\lim _{k \rightarrow \infty} \omega^{k} \rightarrow(-3,2)^{\top}$. Além disso, a sequência $r^{k}$ converge para zero:

$$
r^{k}=\left|\lambda^{k}\left(x_{1}^{k}-x_{2}^{k}\right)\right|+\left|\mu^{k}\left(x_{1}^{k}-x_{2}^{k} \exp x_{2}^{k}\right)\right|=\frac{\left|x_{2}^{k}-x_{2}^{k} \exp x_{2}^{k}\right|}{\left|1-\exp x_{2}^{k}-x_{2}^{k} \exp x_{2}^{k}\right|} \rightarrow 0 .
$$

Dessa forma, $\omega^{k}=(-3,2)^{\top} \in K_{C}\left(x^{k}, r^{k}\right) \forall k \in \mathbb{N}$ e portanto $(-3,2)^{\top} \in \lim \sup _{(x, r) \rightarrow\left(x^{*}, 0\right)} K_{C}(x, r)$ mas $(-3,2)^{\top}$ não pertence a $K\left(x^{*}\right)=\mathbb{R}(-1,1)^{\top}$ e a condição CAKKT regular falha.

$A$ condição $L-A G P$ não vale em $x^{*}=(0,0)$.

Calculemos o conjunto das restrições lineares. Uma vez que a única restrição linear é dada por $h\left(x_{1}, x_{2}\right)=x_{2}-x_{1}$ temos que $\Omega_{L}=\left\{\left(x_{1}, x_{2}\right)^{\top} \in \mathbb{R}^{2}: x_{1}=x_{2}\right\}$.

Defina $x_{1}^{k}:=1 / k, x_{2}^{k}:=x_{1}^{k}, \varepsilon_{1}^{k}:=-\left(x_{2}^{k}-x_{2}^{k} \exp x_{2}^{k}\right)\left(1-\exp x_{2}^{k}-x_{2}^{k} \exp x_{2}^{k}\right)^{-1}, \varepsilon_{2}^{k}:=\varepsilon_{1}^{k}$ e os multiplicadores $\mu^{k}:=-\left(1-\exp x_{2}^{k}-x_{2}^{k} \exp x_{2}^{k}\right)^{-1}$ e $\lambda^{k}:=2-\mu^{k}\left(-\exp x_{2}^{k}-x_{2}^{k} \exp x_{2}^{k}\right)$. Com essas escolhas defina

$$
\omega^{k}:=\lambda^{k}(-1,1)^{\top}+\mu^{k}\left(1,-\exp x_{2}^{k}-x_{2}^{k} \exp x_{2}^{k}\right)^{\top} .
$$

Mostraremos que $\omega^{k} \in N_{\Omega_{N L}\left(x^{k}, \infty\right) \cap \Omega_{L}}\left(x^{k}+\varepsilon^{k}\right)$ para todo $k \in \mathbb{N}$. Claramente, $x^{k}$ e $x^{k}+\varepsilon^{k}$ pertencem a $\Omega_{L}, \mu^{k} \geq 0, \varepsilon^{k} \rightarrow(0,0)^{\top}$ e $\omega^{k}=(-3,2)^{\top} \forall k \in \mathbb{N}$. Para provar que $\omega^{k} \in N_{\Omega_{N L}\left(x^{k}, \infty\right) \cap \Omega_{L}}\left(x^{k}+\varepsilon^{k}\right)$ só falta provar que a escolha do multiplicador $\mu^{k}$ é livre. Como $x_{1}^{k}-x_{1}^{k} \exp x_{1}^{k}<0$ para $x_{1} \neq 0$, temos que $g\left(x_{1}^{k}, x_{2}^{k}\right)<0\left(x_{1}^{k}=x_{2}^{k}\right)$. Assim, o multiplicador associado a $\nabla g\left(x_{1}^{k}, x_{2}^{k}\right)$ é livre se 


$$
\begin{aligned}
g\left(x_{1}, x_{2}^{k}\right)+\left\langle\nabla g\left(x_{1}^{k}, x_{2}^{k}\right),\left(\varepsilon_{1}^{k}, \varepsilon_{2}^{k}\right)\right\rangle=0 \text {, mas pela escolha de } \varepsilon^{k}=\left(\varepsilon_{1}^{k}, \varepsilon_{2}^{k}\right)^{\top} \\
\begin{aligned}
g\left(x_{1}, x_{2}^{k}\right)+\left\langle\nabla g\left(x_{1}^{k}, x_{2}^{k}\right),\left(\varepsilon_{1}^{k}, \varepsilon_{2}^{k}\right)^{\top}\right\rangle & =x_{2}^{k}-x_{2}^{k} \exp x_{2}^{k}+\varepsilon_{1}^{k}+\varepsilon_{2}^{k}\left(-\exp x_{2}^{k}-x_{2}^{k} \exp x_{2}^{k}\right) \\
& =x_{2}^{k}-x_{2}^{k} \exp x_{2}^{k}+\varepsilon_{1}^{k}\left(1-\exp x_{2}^{k}-x_{2}^{k} \exp x_{2}^{k}\right)=0 .
\end{aligned}
\end{aligned}
$$

Portanto podemos escolher $\mu^{k}=-\left(1-\exp x_{2}^{k}-x_{2}^{k} \exp x_{2}^{k}\right)^{-1}$ como multiplicador de $\nabla g\left(x_{1}^{k}, x_{2}^{k}\right)$ e como consequência $\omega^{k}=(-3,2)^{\top} \in N_{\Omega_{N L}\left(x^{k}, \infty\right) \cap \Omega_{L}}\left(x^{k}+\varepsilon^{k}\right)$. Logo $(-3,2)^{\top}=\lim _{k \rightarrow \infty} \omega^{k}$ pertence a $\lim \sup _{(x, \varepsilon) \rightarrow\left(x^{*}, 0\right), x \in \Omega_{L}} N_{\Omega_{N L}(x, \infty) \cap \Omega_{L}}(x+\varepsilon)$ mas $(-3,2)^{\top} \notin K\left(x^{*}\right)$ e consequentemente a condição L-AGP regular falha.

Como Pseudonormalidade implica a Quasinormalidade, do exemplo anterior concluímos que a Quasinormalidade não pode implicar a condição CAKKT regular nem a condição L-AGP regular.

Seguindo a mesma argumentação, para provar que CAKKT regular e L-AGP regular são independentes da Pseudonormalidade e da Quasinormalidade será suficiente provar que a condição CAKKT regular e a condição L-AGP regular não implicam a Quasinormalidade. O seguinte exemplo cumpre esse objetivo.

Exemplo 3.3.4. A condição CAKKT regular e L-AGP regular não implica a Quasinormalidade

Considere $x^{*}=(0,0)^{\top}$ e as restrições de igualdade e desigualdade definidas por

$$
\begin{aligned}
h\left(x_{1}, x_{2}\right) & =x_{1} \\
g_{1}\left(x_{1}, x_{2}\right) & =x_{1}^{3} \\
g_{2}\left(x_{1}, x_{2}\right) & =x_{1} \exp x_{2} .
\end{aligned}
$$

Claramente, $x^{*}$ é viável e ambas restrições são ativas em $x^{*}$. Para todo $x=\left(x_{1}, x_{2}\right) \in \mathbb{R}^{2}$, os gradientes são

$$
\nabla h\left(x_{1}, x_{2}\right)=(1,0)^{\top} \quad \nabla g_{1}\left(x_{1}, x_{2}\right)=\left(3 x_{1}^{2}, 0\right)^{\top} \text { e } \nabla g_{2}\left(x_{1}, x_{2}\right)=\left(\exp x_{2}, x_{1} \exp x_{2}\right)^{\top} .
$$

Assim, o cone $K\left(x^{*}\right)=\left\{\lambda(1,0)^{\top}+\mu_{1}(0,0)^{\top}+\mu_{2}(1,0)^{\top}, \lambda \in \mathbb{R}, \mu_{1} \geq 0, \mu_{2} \geq 0\right\}=\mathbb{R} \times\{0\}$.

A condição CAKKT regular é satisfeita em $x^{*}$.

Seja $\omega^{*} \in \lim \sup _{(x, r) \rightarrow\left(x^{*}, 0\right)} K_{C}(x, r)$. Da definição de limite exterior, existem sequências $\left\{x^{k}\right\},\left\{r^{k}\right\}$ e $\left\{\omega^{k}\right\} \operatorname{com} x^{k}=\left(x_{1}^{k}, x_{2}^{k}\right)^{\top} \rightarrow x^{*}=(0,0)^{\top}, \omega^{k}=\left(\omega_{1}^{k}, \omega_{2}^{k}\right)^{\top} \rightarrow \omega^{*}$ tais que

$$
\omega^{k}=\lambda^{k}(1,0)^{\top}+\mu_{1}^{k}\left(3\left(x_{1}^{k}\right)^{2}, 0\right)^{\top}+\mu_{2}^{k}\left(\exp \left(x_{2}^{k}\right), x_{1}^{k} \exp x_{2}^{k}\right)^{\top} \in K_{C}\left(x^{k}, r^{k}\right)
$$

e

$$
\left.\left|\lambda^{k} x_{1}^{k}\right|+\left|\mu_{1}^{k}\left(x_{1}^{k}\right)^{3}\right|+\mid \mu_{2}^{k} x_{1}^{k} \exp x_{2}^{k}\right) \mid \leq r^{k} \rightarrow 0,
$$

para certos escalares $\lambda^{k}, \mu_{1}^{k}$ e $\mu_{2}^{k}$ com $\mu_{1}^{k} \geq 0$ e $\mu_{2}^{k} \geq 0$. Das expressões 3.3.11 e 3.3.12 temos que $\left|\omega_{2}^{k}=\mu_{2}^{k} x_{1}^{k} \exp x_{2}^{k}\right| \leq r^{k}$. Assim $\omega_{2}^{k} \rightarrow 0$. Claramente, $\omega_{1}^{k}=\lambda^{k}+3 \mu_{1}^{k}\left(x_{1}^{k}\right)^{2} \in \mathbb{R}$, portanto $\omega^{*}=\lim _{k \rightarrow \infty} \omega^{k} \in \mathbb{R} \times\{0\}$ e a condição CAKKT vale em $x^{*}$.

$A$ condição $L-A G P$ regular vale em $x^{*}$.

Calculemos $\Omega_{L}$. Como a única restrição linear é dada pela função $h$ (restrição de igualdade) temos que

$$
\Omega_{L}=\left\{x=\left(x_{1}, x_{2}\right)^{\top} \in \mathbb{R}^{2}: h(x)=0\right\}=\left\{x=\left(x_{1}, x_{2}\right) \in \mathbb{R}^{2}: x_{1}=0\right\}=\{0\} \times \mathbb{R} .
$$

Vamos provar que $N_{\Omega_{N L}(x,-\infty) \cap \Omega_{L}}(x+\varepsilon)$ é semicontínua exteriormente em $\left(x^{*}, 0\right)$ relativamente a $\Omega_{L} \times \mathbb{R}^{2}$. Seja $\omega^{*}=\left(\omega_{1}, \omega_{2}\right)^{\top} \in \lim \sup N_{\Omega_{N L}(x,-\infty) \cap \Omega_{L}}(x+\varepsilon)$ relativamente a $\Omega_{L} \times \mathbb{R}^{2}$. Então, da definição de limite exterior, existem sequências $\left\{x^{k}\right\},\left\{\omega^{k}\right\}$ e $\left\{\varepsilon^{k}\right\}$ em $\mathbb{R}^{2}$ tais que $x^{k} \rightarrow x^{*}, \varepsilon^{k} \rightarrow$ $(0,0), \omega^{k} \rightarrow \omega^{*} \mathrm{e}$

$$
x^{k} \in \Omega_{L} \quad, \quad x^{k}+\varepsilon^{k} \in \Omega_{N L}\left(x^{k},-\infty\right) \cap \Omega_{L} \quad, \quad \omega^{k} \in N_{\Omega_{N L}\left(x^{k},-\infty\right) \cap \Omega_{L}}\left(x^{k}+\varepsilon^{k}\right) .
$$

Para provar que $\omega^{*} \in N_{\Omega_{N L}\left(x^{*},-\infty\right) \cap \Omega_{L}}\left(x^{*}+0\right)=K\left(x^{*}\right)$ analisaremos os diversos casos possíveis. 
Como $x^{k} \in \Omega_{L}$ e $x^{k}+\varepsilon^{k} \in \Omega_{L}$ temos que $x_{1}^{k}=0$ e $\varepsilon_{1}^{k}=0$ e como consequência $g_{1}\left(x_{1}^{k}, x_{2}^{k}\right)=0$ e $g_{2}\left(x_{1}^{k}, x_{2}^{k}\right)=0$ para todo $k \in \mathbb{N}$. Percebamos que independentemente do valor de $\varepsilon_{2}^{k}$ temos que

$$
\left\langle\nabla g_{1}\left(x_{1}^{k}, x_{2}^{k}\right),\left(\varepsilon_{1}^{k}, \varepsilon_{2}^{k}\right)^{\top}\right\rangle=0 \quad \text { e } \quad\left\langle\nabla g_{2}\left(x_{1}^{k}, x_{2}^{k}\right),\left(\varepsilon_{1}^{k}, \varepsilon_{2}^{k}\right)^{\top}\right\rangle=0,
$$

pois quando $x_{1}^{k}=\varepsilon_{1}^{k}=0$ temos que

$$
\left\langle\nabla g_{1}\left(x_{1}^{k}, x_{2}^{k}\right),\left(\varepsilon_{1}^{k}, \varepsilon_{2}^{k}\right)^{\top}\right\rangle=\varepsilon_{1}^{k}\left(3\left(x_{1}^{k}\right)^{2}\right)+\varepsilon_{2}^{k}(0)=0 .\left(3\left(x_{1}^{k}\right)^{2}\right)+\varepsilon_{2}^{k} \cdot 0=0
$$

e

$$
\left\langle\nabla g_{2}\left(x_{1}^{k}, x_{2}^{k}\right),\left(\varepsilon_{1}^{k}, \varepsilon_{2}^{k}\right)^{\top}\right\rangle=\varepsilon_{1}^{k}\left(\exp x_{2}^{k}\right)+\varepsilon_{2}^{k}\left(x_{1}^{k} \exp x_{2}^{k}\right)=0 . \exp x_{2}^{k}+\varepsilon_{2}^{k} .0=0 .
$$

Assim, existem $\mu_{1}^{k} \geq 0, \mu_{2}^{k} \geq 0$ (não necessariamente nulos) tais que

$$
\omega^{k}=\lambda^{k} \nabla h\left(x_{1}^{k}, x_{2}^{k}\right)+\mu_{1}^{k} \nabla g_{1}\left(x_{1}^{k}, x_{2}^{k}\right)+\mu_{2}^{k} \nabla g_{2}\left(x_{1}^{k}, x_{2}^{k}\right) \in N_{\Omega_{N L}\left(x^{k},-\infty\right) \cap \Omega_{L}}\left(x^{k}+\varepsilon^{k}\right) .
$$

Já que $x_{1}^{k}=0$ temos que $\nabla h\left(x_{1}, x_{2}\right)=(1,0)^{\top}, \nabla g_{1}\left(x_{1}^{k}, x_{2}^{k}\right)=(0,0)^{\top}$ e $\nabla g_{2}\left(x_{1}^{k}, x_{2}^{k}\right)=\left(\exp x_{2}^{k}, 0\right)^{\top}$. Assim, $\omega_{2}^{k}=0$ para todo $k \in \mathbb{N}$. Como consequência $\omega^{*}=\lim _{k \rightarrow \infty} \omega^{k} \in \mathbb{R} \times\{0\}=K\left(x^{*}\right)$, provando que a condição de qualificação L-AGP é satisfeita em $x^{*}=(0,0)^{\top}$.

A Quasinormalidade não vale em $x^{*}$.

Para cada $k \in \mathbb{N}$, defina $x_{1}^{k}:=1 / k, x_{1}^{k}:=x_{2}^{k}, \lambda:=0, \mu_{1}:=1$ e $\mu_{2}:=0$. Com essas escolhas temos que $\lambda \nabla h\left(x^{*}\right)+\mu_{1} \nabla g_{1}\left(x^{*}\right)+\mu_{2} \nabla g_{2}\left(x^{*}\right)=0 .(1,0)^{\top}+1 .(0,0)^{\top}+0 .(1,0)^{\top}=(0,0) \mathrm{e}$ $\mu_{1} g_{1}\left(x_{1}^{k}, x_{2}^{k}\right)=\left(x_{1}^{k}\right)^{3}>0$ para todo $k \in \mathbb{N}$. Assim, a Quasinormalidade falha em $x^{*}$.

A Figura 3.7 mostra os resultados obtidos nessa seção. Oferecemos o panorama mais completo de condições de qualificação com claras implicações algorítmicas.

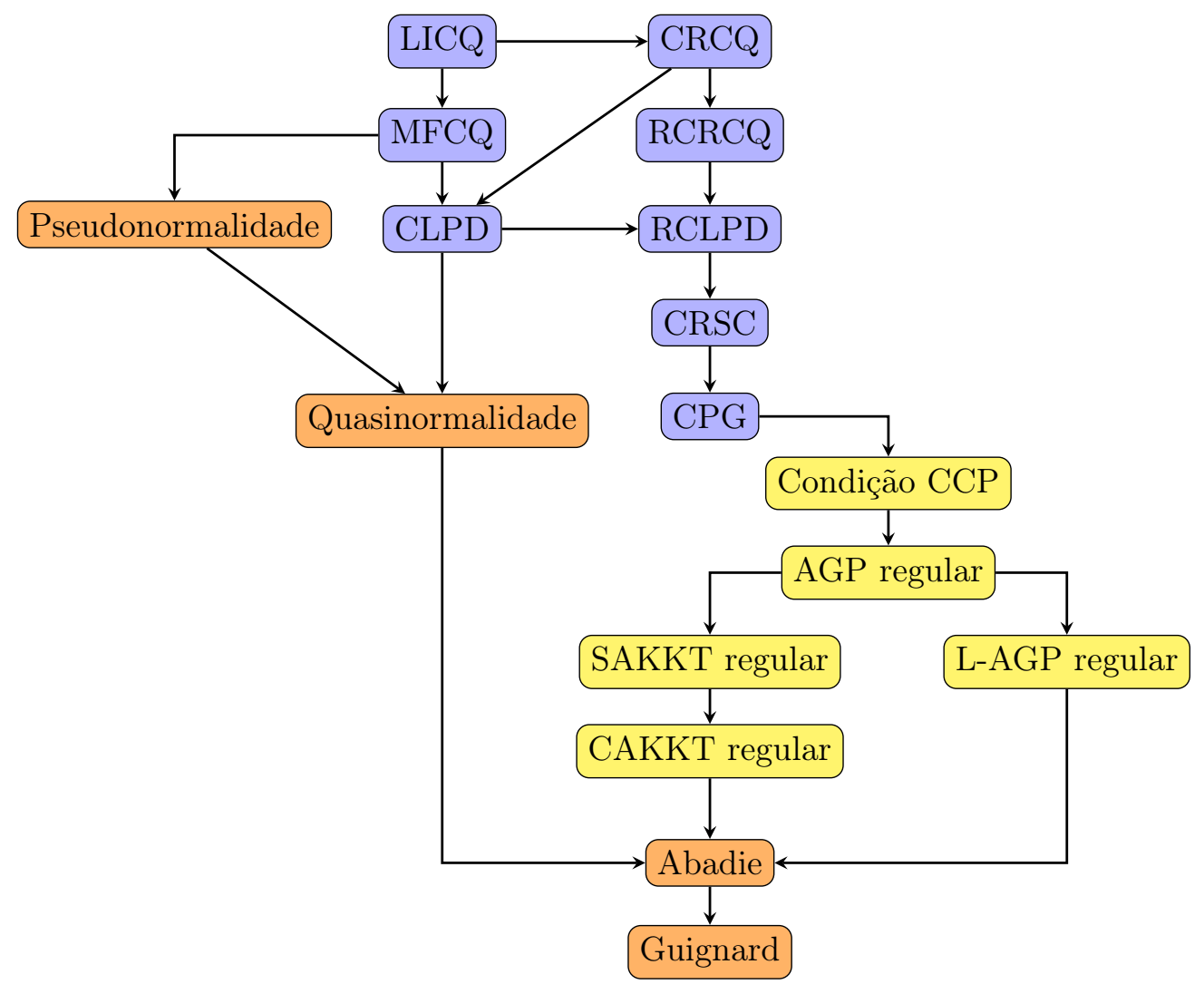

Figura 3.7: Relações das novas condições de qualficação (CCP, AGP regular, SAKKT regular, CAKKT regular e L-AGP regular) com as CQs conhecidas na literatura. 
52 CONDIÇÕES DE QUALIFICAÇÃO ASSOCIADAS COM AS CONDIÇÕES SEQUENCIAIS DE OTIMALIDADE DE PRIMEIRA ORDEM 


\section{Capítulo 4}

\section{Condição Sequencial de Segunda Ordem AKKT2 e condições de qualificação}

As condições sequenciais de otimalidade têm demonstrado ser uma ferramenta útil no estudo de algoritmos práticos. Por exemplo, têm sido usadas na análise global de convergência de algoritmos, como os métodos de lagrangiano aumentado, os métodos de restauração inexata, e alguns métodos de programação quadrática sequencial sob condições de qualificação bem mais fracas que as usuais, como a condição CPLD ou a condição CPG (ver (AHSS12a, AHSS12b)). Além disso, elas fornecem um fundamento teórico dos diferentes critérios de parada associados a algoritmos práticos e têm servido como modelo no desenho de novos algoritmos práticos, (BHM14, BBM15).

Sob esse paradigma, gostaríamos de definir condições sequenciais de otimalidade apropriadas para o estudo de algoritmos com convergência a pontos estacionários de segunda ordem, que desempenhem o mesmo papel de unificação que desempenha AKKT, e outras condições sequenciais de primeira ordem, na análise de convergência de muitos algoritmos.

Muitos algoritmos com convergência a pontos estacionários de segunda ordem (i.e. pontos onde WSONC vale) têm sido propostos na literatura. Andreani, Birgin, Martinez e Schuverdt (ABMS10), ver também (AMS07), usaram um método de curvatura negativa de segunda ordem para minimização em caixas aplicados a certa classe de funções que não possuem segundas derivadas contínuas. Byrd, Schnabel e Shultz (BSS87) propuseram um método de programação quadrática sequencial (SQP), no qual a convergência a pontos estacionários de segunda ordem é obtida usando correções de segunda ordem. Coleman, Liu e Yuan (CLY02) usaram também um método SQP com uma função de penalidade quadrática para minimização com restrições de igualdade. Conn, Gould, Orban e Toint (CGOT98) utilizaram um método de barreira logarítmica para problemas de minimização com desigualdades e com restrições de igualdades lineares. Di Pillo, Lucidi e Palagi (DLP05) definiram um algoritmo primal-dual para problemas de minimização com restrições de desigualdades e tomaram vantagem da equivalência entre o problema original com restrições e a minimização sem restrições de uma função lagrangiano aumentado. Eles usaram uma técnica de busca curvilinear usando a informação sobre a não convexidade dessa função de lagrangiano aumentado. Facchinei e Lucidi (FL98) usaram direções de curvatura negativa no contexto de minimização com restrições de desigualdades. Recentemente, Gill, Kungurtsev e Robinson em (GKR13) utilizaram uma variante do método SQP, o método SQP regularizado definido em (GR13). Esse método é baseado em uma busca linear flexível junto com uma direção definida através de uma solução de um subproblema de programação quadrática de uma função estritamente convexa e, quando existe, uma direção de curvatura negativa para certo lagrangiano aumentado primal-dual. Em (MP03), Morguerza e Prieto usaram um algoritmo de pontos interiores para problemas não convexos juntamente com direções de curvatura negativa. A convergência a pontos estacionários de segunda ordem para métodos de regiões de confiança com restrições convexas é estudada em detalhes por Conn, Gould e Toint em (CGT00).

Nossa contribuição neste capítulo é introduzir a primeira condição sequencial de otimalidade que tem em consideração não só a informação de primeira ordem mas também a informação de 
segunda ordem, está condição é chamada de AKKT2 (second-order approximate KKT condition). Na seção 4.1, definiremos a condição AKKT2 e mostraremos que AKKT2 é uma genuína condição de otimalidade e que vários algoritmos com convergência a pontos estacionários de segunda ordem geram sequências cujo pontos limites cumprem esta condição. Está condição de otimalidade é forte, no sentido, que ela implica a proposição (2.0.14) para CQ mais fracas que a condição de qualificação LICQ. Na seção 4.2 definimos uma nova condição chamada CCP2, qual é a CQ mínima associada à AKKT2 e na seção 4.3 e mostramos as relações existentes entre CCP2 e outras CQs conhecida na literatura, como por exemplo MFCQ $+\mathrm{WCR}, \mathrm{RCRCQ}$ e a condição de Baccari-Trad. Na última seção, seção 4.4. fornecemos outro motivo do porque WSONC é a condições de segunda ordem adequada quando analisamos o comportamento de algoritmos. Ver (AHRS15).

Para terminar, destacamos o seguinte lema de grande utilidade nas demonstrações deste capítulo.

Lema 4.0.7. (Ber82) Sejam $P \in \operatorname{Sym}(n)$ e vetores $a_{1}, \ldots, a_{r} \in \mathbb{R}^{n}$. Defina o seguinte subespaço $\mathcal{C}:=\left\{d \in \mathbb{R}^{n}:\left\langle a_{j}, d\right\rangle=0\right.$ para todo $\left.j \in\{1, \ldots, r\}\right\}$. Suponha que $P(v, v)>0$ para todo $v \in \mathcal{C}$. Então, existem $\left\{c_{j} \in \mathbb{R}_{+}, j \in\{1, \ldots, r\}\right\}$ tais que $P+\sum_{j=1}^{r} c_{j} a_{j} a_{j}^{T} \succ 0$.

\subsection{Condição Sequencial de Segunda Ordem: AKKT2}

Nesta seção introduziremos uma nova condição sequencial de otimalidade, chamada de AKKT2, apropriada para a análise de algoritmos com convergência a pontos estacionários de segunda ordem. Como toda condição sequencial, ela deve satisfazer três propriedades: (i) deve ser realmente uma condição de otimalidade, independentemente de qualquer condição de qualificação; (ii) ela deve ser o mais forte possível, em nosso caso, ela deve implicar (2.0.14) para condições de qualificação fracas (na subseção 4.1.1 provaremos essas propriedades); (iii) ela deve ser gerada por algoritmos práticos. Na subseção 4.1.2. mostraremos que o método de lagrangiano aumentado de (ABMS10), o método SQP regularizado de (GKR13) e o método de regiões de confiança de (DV97) geram sequências onde os pontos limites satisfazem essa nova condição sequencial de otimalidade.

\subsubsection{Condição sequencial AKKT2}

Nesta subseção procederemos a definir nossa condição sequencial de segunda ordem.

Definição 4.1.1. Dizemos que um ponto viável $x^{*} \in \Omega$ é um ponto AKKT2 (do inglês approximate second-order KKT) para o problema (2.0.3) se existem sequencias $\left\{x^{k}\right\} \subset \mathbb{R}^{n},\left\{\lambda^{k}\right\} \subset \mathbb{R}^{m},\left\{\eta^{k}\right\} \subset$ $\mathbb{R}^{m},\left\{\mu^{k}\right\} \subset \mathbb{R}_{+}^{p},\left\{\theta^{k}\right\} \subset \mathbb{R}_{+}^{p},\left\{\delta_{k}\right\} \subset \mathbb{R}_{+}$com $\mu_{j}^{k}=0$ para $j \notin J\left(x^{*}\right), \theta_{j}^{k}=0$ para $j \notin J\left(x^{*}\right)$ tais que $x^{k} \rightarrow x^{*}, \delta_{k} \rightarrow 0$

$$
\lim _{k \rightarrow \infty} \nabla_{x} L\left(x^{k}, \lambda^{k}, \mu^{k}\right)=\lim _{k \rightarrow \infty} \nabla f\left(x^{k}\right)+\sum_{i=1}^{m} \lambda_{i}^{k} \nabla h_{i}\left(x^{k}\right)+\sum_{j \in J\left(x^{*}\right)} \mu_{j}^{k} \nabla g_{j}\left(x^{k}\right)=0
$$

$e$

$$
\nabla_{x}^{2} L\left(x^{k}, \lambda^{k}, \mu^{k}\right)+\sum_{i=1}^{m} \eta_{i}^{k} \nabla h_{i}\left(x^{k}\right) \nabla h_{i}\left(x^{k}\right)^{T}+\sum_{j \in J\left(x^{*}\right)} \theta_{j}^{k} \nabla g_{j}\left(x^{k}\right) \nabla g_{j}\left(x^{k}\right)^{T} \succeq-\delta_{k} \mathbb{I}
$$

para $k \in \mathbb{N}$ suficientemente grande.

A sequência $\left\{x^{k}\right\}$ é chamada de sequência AKKT2 e $x^{*}$ é chamado de ponto AKKT2.

\section{AKKT2 é uma condição de otimalidade}

Para provar que a condição AKKT2 é uma condição de otimalidade usaremos o seguinte lema. 
Lema 4.1.1 ((ABMS10, AMS07)). Sejam $\bar{f}: \mathbb{R}^{n} \rightarrow \mathbb{R}, \bar{g}_{j}: \mathbb{R}^{n} \rightarrow \mathbb{R}$ para $j \in\{1, \ldots, p\}$ funções suficientemente diferenciáveis em uma vizinhança de $\bar{x}$. Defina

$$
\bar{F}(x):=\bar{f}(x)+\frac{1}{2} \sum_{j=1}^{p} \max \left\{0, \bar{g}_{j}(x)\right\}^{2}
$$

para todo $x$ em uma vizinhança aberta de $\bar{x}$. Suponha que $\bar{x}$ é uma minimizador local de $\bar{F}$. Então a matriz definida como

$$
H(x):=\nabla^{2} \bar{f}(x)+\sum_{j=1}^{p} \max \left\{0, \bar{g}_{j}(x)\right\} \nabla^{2} \bar{g}_{j}(x)+\sum_{j: \bar{g}(\bar{x}) \geq 0} \nabla \bar{g}_{j}(x) \nabla \bar{g}_{j}(x)^{T}
$$

é semi-definida positiva em $\bar{x}$.

Teorema 4.1.2. Seja $x^{*}$ um minimizador local de (2.0.3) então $x^{*}$ satisfaz a condição AKKT2.

Demonstração. Devido a que $x^{*}$ é um minimizador local de (2.0.3), existe um escalar $\varepsilon>0$ tal que $f\left(x^{*}\right) \leq f(x)$ para todo $x$ viável tal que $\left\|x-x^{*}\right\| \leq \varepsilon$. Assim, $x^{*}$ é a única solução de

$$
\text { minimizar } f(x)+\frac{1}{4}\left\|x-x^{*}\right\|^{4} \text { sujeito a } h(x)=0, \quad g(x) \leq 0, \quad x \in \mathbb{B}\left(x^{*}, \varepsilon\right) .
$$

Seja $\left\{\rho_{k}\right\} \subset \mathbb{R}_{+}$tal que $\rho_{k} \uparrow \infty$. Para cada $\rho_{k}$ e $\varepsilon>0$, considere o seguinte problema

$$
\text { minimizar } f(x)+\frac{1}{4}\left\|x-x^{*}\right\|^{4}+\frac{1}{2} \rho_{k}\left(\sum_{i=1}^{m} h_{i}(x)^{2}+\sum_{j=1}^{p} \max \left\{g_{j}(x), 0\right\}^{2}\right) \text { s. a } x \in \mathbb{B}\left(x^{*}, \varepsilon\right) \text {. }
$$

Seja $x^{k}$ solução do subproblema 4.1.4). Seguindo a mesma linha da demonstração do Teorema 2.4.1. temos que $\left\{x^{k}\right\}$ está bem definida, $x^{k} \rightarrow x^{*}$ e para $k$ suficientemente grande $x^{k}$ pertence ao interior de $\mathbb{B}\left(x^{*}, \varepsilon\right)$. Desta forma, o gradiente da função objetivo de 4.1.4 deve ser zero em $x^{k}$ :

$$
\nabla f\left(x^{k}\right)+\sum_{i=1}^{m} \rho_{k} h_{i}\left(x^{k}\right) \nabla h_{j}\left(x^{k}\right)+\sum_{j=1}^{p} \rho_{k} \max \left\{0, g_{j}\left(x^{k}\right)\right\} \nabla g_{j}\left(x^{k}\right)+\left\|x^{k}-x^{*}\right\|^{3}\left(x^{k}-x^{*}\right)=0 .
$$

Do Lema 4.1.1 $\operatorname{com} \bar{F}(x)=f(x)+\frac{1}{2} \rho_{k} \sum_{i=1}^{m} h_{i}(x)^{2}+\frac{1}{4}\left\|x-x^{*}\right\|^{4}, \bar{g}_{j}=\sqrt{\rho_{k}} g_{j}(x)$ para $j \in\{1, . ., p\}$ e $\bar{x}=x^{k}$, obtemos que:

$$
\begin{gathered}
\nabla^{2} f\left(x^{k}\right)+\sum_{i=1}^{m} \rho_{k} h_{i}\left(x^{k}\right) \nabla^{2} h_{j}\left(x^{k}\right)+\sum_{j=1}^{p} \rho_{k} \max \left\{g_{j}\left(x^{k}\right), 0\right\} \nabla^{2} g_{j}\left(x^{k}\right)+\sum_{i=1}^{m} \rho_{k} \nabla h_{i}\left(x^{k}\right) \nabla h_{j}\left(x^{k}\right)^{T}+ \\
\sum_{j: g_{j}\left(x^{k}\right) \geq 0} \rho_{k} \nabla g_{j}\left(x^{k}\right) \nabla g_{j}\left(x^{k}\right)^{T}+2\left(x^{k}-x^{*}\right)\left(x^{k}-x^{*}\right)^{*}+\left\|x^{k}-x^{*}\right\|^{2} \mathbb{I} \succeq 0
\end{gathered}
$$

Defina $\lambda_{i}^{k}:=\rho_{k} h_{i}\left(x^{k}\right), \eta_{i}^{k}:=\rho_{k}$ para $i \in\{1, \ldots, m\}, \mu_{j}^{k}:=\rho_{k} \max \left\{g_{j}\left(x^{k}\right), 0\right\}$ para $j \in\{1, . ., p\}$, $\theta_{j}^{k}=\rho_{k}$ sempre que $g_{j}\left(x^{k}\right) \geq 0$ e $\theta_{j}^{k}=0$ no caso contrário. Finalmente defina $\delta_{k}:=3\left\|x^{k}-x^{*}\right\|^{2}$. Obviamente $\delta_{k} \rightarrow 0$ e para $k$ suficientemente grande, $\mu_{j}^{k}=0$ para todo $j \notin J\left(x^{*}\right)$ e $\theta_{j}^{k}=0$ para todo $j \notin J\left(x^{*}\right)$. Assim, das expressões 4.1.5 e 4.1.6, concluímos que 4.1.1) e 4.1.2 são satisfeitas e portanto $x^{*}$ satisfaz a condição AKKT2.

\section{A força da condição AKKT2}

A condição AKKT2 é uma condição de otimalidade forte, no sentido, que ele implica (2.0.14) para condições de qualificação fracas. Em esta subseção provaremos, sob a condição MFCQ+WCR, que todo ponto AKKT2 satisfaz a condição WSONC, ver Proposição 4.1.4. Posteriormente, pro- 
varemos que a condiçao CRCQ e a condição RCRCQ podem também ser usadas como CQ2 na expressão (2.0.14), ver Proposição 4.3.5. Como consequência a condição RCRCQ também pode ser usada na análise de convergência de algoritmos práticos.

Para provar que sob a condição MFCQ +WCR, todo ponto AKKT2 satisfaz a condição WSONC, começaremos com a definição da condição WCR (do inglês weak constant rank) introduzida por Andreani, Martínez e Schuverdt em (AMS07).

Definição 4.1.2. Seja $x^{*}$ um ponto viável. A condição WCR vale em $x^{*}$ se existe uma vizinhança $V$ de $x^{*}$ tal que o posto de $\left\{\nabla h_{i}(x), \nabla g_{j}(x): i \in\{1, \ldots, m\}, j \in J\left(x^{*}\right)\right\}$ é constante para todo $x \in V$.

A seguinte propriedade da condição WCR é importante para as demonstrações futuras.

Lema 4.1.3. (AMS07) Seja $d \in C^{\mathcal{W}}\left(x^{*}\right)$. Então para toda sequência $\left\{x^{k}\right\} \subset \mathbb{R}^{n}$ com $x^{k} \rightarrow x^{*}$, existe uma sequência $\left\{d^{k}\right\} \subset \mathbb{R}^{n}$ com $d^{k} \rightarrow d$ tal que, $\left\langle\nabla h_{i}\left(x^{k}\right), d^{k}\right\rangle=0$ para $i \in\{1, \ldots, m\}$ e $\left\langle\nabla g_{j}\left(x^{k}\right), d^{k}\right\rangle=0$ para $j \in J\left(x^{*}\right)$.

Proposição 4.1.4. Seja $x^{*} \in \Omega$ tal que a condição AKKT2 vale. Se a condição de qualificação $M F C Q+W C R$ se cumpre em $x^{*}$ então a WSONC é satisfeita em $x^{*}$.

Demonstração. Pela definição de AKKT2 existem sequências $\left\{x^{k}\right\},\left\{\mu^{k}\right\},\left\{\delta_{k}\right\},\left\{\theta^{k}\right\}$ com $\mu_{j}^{k}=0$ e $\theta_{j}^{k}=0$ para todo $j \notin J\left(x^{*}\right)$ tais que $x^{k} \rightarrow x^{*}, \delta_{k} \rightarrow 0$ e

a) $\varepsilon^{k}:=\nabla f\left(x^{k}\right)+\sum_{i=1}^{m} \lambda_{i}^{k} \nabla h_{i}\left(x^{k}\right)+\sum_{j \in J\left(x^{*}\right)} \mu_{j}^{k} \nabla g_{j}\left(x^{k}\right) \rightarrow 0 ;$

b) $M^{k}:=\nabla_{x}^{2} L\left(x^{k}, \lambda^{k}, \mu^{k}\right)+\sum_{i=1}^{m} \eta_{i}^{k} \nabla h_{i}\left(x^{k}\right) \nabla h_{i}\left(x^{k}\right)^{T}+\sum_{j \in J\left(x^{*}\right)} \theta_{j}^{k} \nabla g_{j}\left(x^{k}\right) \nabla g_{j}\left(x^{k}\right)^{T} \succeq-\delta_{k} \mathbb{I}$.

Pela condição de Mangasarian-Fromovitz, a sequência $\left\{\left(\lambda^{k}, \mu^{k}\right)\right\}$ é limitada. Para provar isso, suponha por contradição que a sequência $\left\{\left(\lambda^{k}, \mu^{k}\right)\right\}$ é ilimitada. Defina a sequência $\left\{A_{k}\right\}$ como $\max \left\{\left|\lambda_{i}^{k}\right|, \mu_{j}^{k}: i \in\{1, \ldots, m\}, j \in J\left(x^{*}\right)\right\}$. Claramente, $\left\{A_{k}\right\}$ vai para o infinito. Dividindo por $A_{k}$ a relação $\varepsilon^{k}=\nabla f\left(x^{k}\right)+\sum_{i=1}^{m} \lambda_{i}^{k} \nabla h_{i}\left(x^{k}\right)+\sum_{j \in J\left(x^{*}\right)} \mu_{j}^{k} \nabla g_{j}\left(x^{k}\right)$, e tomando uma subsequência adequada tal que $\left\{\left(\lambda^{k} / A_{k}, \mu^{k} / A_{k}\right)\right\}$ converge temos que $\sum_{i=1}^{m} \lambda_{i}^{*} \nabla h_{i}\left(x^{*}\right)+\sum_{j \in J\left(x^{*}\right)} \mu_{j}^{*} \nabla g_{j}\left(x^{*}\right)=0$ para certos $\lambda^{*} \in \mathbb{R}^{n}$ e $\mu^{*} \geq 0$ não todos nulos, que é uma contradição com MFCQ. Portanto, a sequência $\left\{\left(\lambda^{k}, \mu^{k}\right)\right\}$ é limitada. Assim, sem perda de generalidade podemos supor que $\left\{\left(\lambda^{k}, \mu^{k}\right)\right\}$ converge para $\left(\mu^{*}, \lambda^{*}\right)$. Defina $\mu_{j}^{*}=0$ para todo $j \notin J\left(x^{*}\right)$. Tomando limite no item (a), temos que $\left(x^{*}, \mu^{*}, \lambda^{*}\right)$ satisfaz as condições KKT. Procederemos a provar que WSONC vale em $x^{*}$ com multiplicadores $\left(\mu^{*}, \lambda^{*}\right)$.

Seja $d \in C^{\mathcal{W}}\left(x^{*}\right)$, pelo Lema 4.1 .3 , existe uma sequência $d^{k}$ tal que $d^{k} \rightarrow d,\left\langle\nabla h_{i}\left(x^{k}\right), d^{k}\right\rangle=0$, $i \in\{1, \ldots, m\}$ e $\left\langle\nabla g_{j}\left(x^{k}\right), d^{k}\right\rangle=0$, para todo $j \in J\left(x^{*}\right)$. Assim, avaliando a forma quadrática $\left\langle d^{k}, M^{k} d^{k}\right\rangle$ e usando o item (b) obtemos que:

$$
\left\langle\nabla_{x}^{2} L\left(x^{k}, \lambda^{k}, \mu^{k}\right) d^{k}\right\rangle+\sum_{i=1}^{m} \eta_{i}^{k}\left(\left\langle\nabla h_{i}\left(x^{k}\right), d^{k}\right\rangle\right)^{2}+\sum_{j \in J\left(x^{*}\right)} \theta_{j}^{k}\left(\left\langle\nabla g_{j}\left(x^{k}\right), d^{k}\right\rangle\right)^{2} \geq-\delta_{k}\left\|d^{k}\right\|^{2}
$$

o que implica que

$$
\nabla_{x}^{2} L\left(x^{k}, \lambda^{k}, \mu^{k}\right)\left(d^{k}, d^{k}\right)=\left\langle\nabla_{x}^{2} L\left(x^{k}, \lambda^{k}, \mu^{k}\right) d^{k}\right\rangle \geq-\delta_{k}\left\|d^{k}\right\|^{2} .
$$

Finalmente, tomando limite em 4.1.8, temos que

$$
\left\langle d^{*},\left(\nabla^{2} f\left(x^{*}\right)+\sum_{i=1}^{m} \lambda_{i}^{*} \nabla^{2} h_{i}\left(x^{*}\right)+\sum_{j \in J\left(x^{*}\right)} \mu_{j}^{*} \nabla^{2} g_{j}\left(x^{*}\right)\right) d^{*}\right\rangle \geq 0,
$$

como queríamos provar. 
Claramente, da definição de AKKT2, a condição AKKT2 implica a condição sequencial AKKT. O seguinte exemplo mostra que AKKT2 é estritamente mais forte que a condição AKKT.

Exemplo 4.1.1. Consideremos $f\left(x_{1}, x_{2}\right)=-x_{1}-x_{2}, g\left(x_{1}, x_{2}\right)=x_{1}^{2} x_{2}^{2}-1$ e $x=(1,1)^{\top}$.

Primeiro, mostraremos que AKKT vale em $x^{*}=(1,1)^{\top}$. Como os gradientes das funções são $\nabla f\left(x_{1}, x_{2}\right)=(-1,-1)$ e $\nabla g\left(x_{1}, x_{2}\right)=2 x_{1} x_{2}\left(x_{2}, x_{1}\right)$, as condições KKT valem em $x^{*} \operatorname{com} \mu=1 / 2$ como multiplicador. Agora, provaremos que AKKT2 falha. Suponha pelo contrário que não, assim 4.1.1 e 4.1.2 se cumprem. Defina $d^{k}:=\left(x_{1}^{k},-x_{2}^{k}\right)^{\top}$ onde $\left\{\left(x_{1}^{k}, x_{2}^{k}\right)^{\top}\right\}$ é a sequência dada pela definição de AKKT2. Desde que $\left\langle\nabla g\left(x_{1}^{k}, x_{2}^{k}\right), d^{k}\right\rangle=0$ para todo $k \in \mathbb{N}$, segue de 4.1.2 que:

$$
\mu^{k} \nabla^{2} g\left(x_{1}^{k}, x_{2}^{k}\right)\left(d^{k}, d^{k}\right)+\delta_{k}\left\|d^{k}\right\|^{2} \geq 0
$$

para alguns $\mu^{k} \geq 0$ e $\delta_{k} \geq 0$ com $\delta_{k} \rightarrow 0$. Do cálculo temos que $\nabla^{2} g\left(x_{1}^{k}, x_{2}^{k}\right)\left(d^{k}, d^{k}\right)=-4\left(x_{1}^{k} x_{2}^{k}\right)^{2}$. Assim, substituindo em (4.1.10), temos $-4 \mu^{k}\left(x_{1}^{k} x_{2}^{k}\right)^{2}+\delta_{k}\left\|d^{k}\right\|^{2} \geq 0$ para todo $k \in \mathbb{N}$. Mas isso é impossível porque por 4.1.1): $\nabla f\left(x_{1}, x_{2}\right)+\mu^{k} \nabla g\left(x_{1}, x_{2}\right) \rightarrow 0$ implica que $-1+2 \mu^{k} x_{1}^{k}\left(x_{2}^{k}\right)^{2} \rightarrow 0$ e como consequência $2 \mu^{k}\left(x_{1}^{k} x_{2}^{k}\right)^{2} \rightarrow 1$ e $-4 \mu^{k}\left(x_{1}^{k} x_{2}^{k}\right)^{2}+\delta_{k}\left\|d^{k}\right\|^{2} \rightarrow-2$.

\subsubsection{Algoritmos práticos que geram sequências AKKT2}

Nesta seção provaremos que existem algoritmos que geram sequencias cujos pontos limites satisfazem a condição AKKT2. Como exemplos, podemos mencionar o lagrangiano aumentado proposto em (ABMS10) (ver também (AMS07)), o método regularizado SQP de Gill, Kungurtsev e Robinson (GKR13) e o método de regiões de confiança de Dennis e Vicente (DV97).

\section{Método de lagrangiano aumentado com convergência para pontos estacionários de segunda ordem.}

Mostraremos que o método de lagrangiano aumentado proposto por (ABMS10) (ver (AMS07)) gera sequências AKKT2. Antes de começar a análise do método de lagrangiano aumentado de (AMS07) para o problema 2.0.3) (que é equivalente ao método proposto em (ABMS10) quando a restrição de caixa é todo o $\mathbb{R}^{n}$ ) vejamos algumas notações adicionais. Considere a seguinte função lagrangiana aumentada

$$
L_{\rho}(x, \lambda, \mu):=f(x)+\frac{\rho}{2}\left(\sum_{i=1}^{m}\left[h_{i}(x)+\frac{\lambda_{i}}{\rho}\right]^{2}+\sum_{j=1}^{p}\left[\max \left\{0, g_{j}(x)+\frac{\mu_{j}}{\rho}\right\}\right]^{2}\right),
$$

para todo $x \in \mathbb{R}^{n}, \rho>0, \lambda \in \mathbb{R}^{m}$ e $\mu \in \mathbb{R}_{+}^{p}$. A função $L_{\rho}$ possui primeiras derivadas contínuas com respeito à variável $x$, mas a segunda derivada não está definida nos pontos $x$ tais que $g_{j}(x)+\mu_{j} / \rho=0$. Assim, para superar essa dificuldade, em (AMS07) os autores definiram:

$$
\begin{aligned}
& \bar{\nabla}^{2}\left(\max \left\{0, g_{i}(x)+\frac{\mu_{i}}{\rho}\right\}\right)^{2}:=\nabla^{2}\left(g_{i}(x)+\frac{\mu_{i}}{\rho}\right)^{2} \text { se } g_{i}(x)+\frac{\mu_{i}}{\rho}=0 \\
& \bar{\nabla}^{2}\left(\max \left\{0, g_{i}(x)+\frac{\mu_{i}}{\rho}\right\}\right)^{2}:=\nabla^{2}\left(\max \left\{0, g_{i}(x)+\frac{\mu_{i}}{\rho}\right\}\right)^{2} \text { caso contrário. }
\end{aligned}
$$

Agora procederemos a descrever o algoritmo. Ver Algoritmo 1.

De 4.1.14 temos que qualquer ponto limite $\left\{x^{k}\right\}$ satisfaz a condição AKKT2. De fato, seja $x^{*}$ qualquer ponto limite da sequência $\left\{x^{k}\right\}$. Pela demonstração de (AMS07, Teorema 4.1), temos que, para $k$ suficientemente grande, a expressão 4.1.14 é equivalente a (AMS07, Teorema 4.1)

$$
\left\|\nabla L\left(x^{k}, \hat{\lambda}^{k}, \hat{\mu}^{k}\right)\right\| \leq \varepsilon_{k}
$$


Algorithm 1 (AMS07, Algoritmo 4.1).

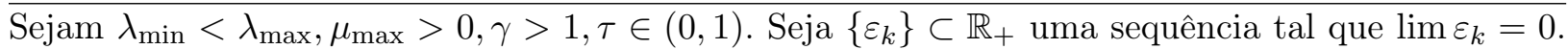
Escolha $\lambda_{i}^{1} \in\left[\lambda_{\min }, \lambda_{\max }\right]$ com $i \in\{1, \ldots, m\}, \mu_{j}^{1} \in\left[0, \mu_{\max }\right]$ para $j \in\{1, \ldots, p\}$ e $\rho_{1}>0$. Seja $x^{0} \in \mathbb{R}^{n}$ um ponto inicial arbitrário. Defina $V^{0}:=g\left(x^{0}\right)^{+}$. Inicialize com $k=1$.

1. Encontre um minimizador aproximado $x^{k}$ de $L_{\rho_{k}}\left(x, \lambda^{k}, \mu^{k}\right)$. As condições para $x^{k}$ são:

$$
\left\|\nabla L_{\rho_{k}}\left(x^{k}, \lambda^{k}, \mu^{k}\right)\right\| \leq \varepsilon_{k} \quad \text { e } \quad \bar{\nabla}^{2} L_{\rho_{k}}\left(x^{k}, \lambda^{k}, \mu^{k}\right) \succeq-\varepsilon_{k} \mathbb{I}
$$

2. Defina $V_{j}^{k}:=\max \left\{g_{j}\left(x^{k}\right),-\mu_{j}^{k} / \rho_{k}\right\}$ para $j \in\{1, \ldots, p\}$.

Se $\max \left\{\left\|h\left(x^{k}\right)\right\|_{\infty},\left\|V^{k}\right\|_{\infty}\right\} \leq \tau \max \left\{\left\|h\left(x^{k-1}\right)\right\|_{\infty},\left\|V^{k-1}\right\|_{\infty}\right\}$ coloque $\rho_{k+1}=\rho_{k}$, caso contrário $\rho_{k+1}=\gamma \rho_{k}$;

3. Calcule $\lambda_{i}^{k+1} \in\left[\lambda_{\min }, \lambda_{\max }\right]$ para todo $i \in\{1, \ldots, m\}$ e $\mu_{j}^{k+1} \in\left[0, \mu_{\max }\right], j \in\{1, \ldots, p\}$. Faça $k \leftarrow k+1$ e ir para o Passo 1 .

$\mathrm{e}$

$$
\nabla^{2} L\left(x^{k}, \hat{\lambda}^{k}, \hat{\mu}^{k}\right)+\rho_{k} \sum_{i=1}^{m} \nabla h_{i}(x) \nabla h_{i}(x)^{T}+\rho_{k} \sum_{j \in J\left(x^{*}\right)} \nabla g_{j}(x) \nabla g_{j}(x)^{T} \succeq-\varepsilon_{k} \mathbb{I},
$$

onde $\hat{\lambda}_{i}^{k}:=\lambda_{i}^{k}+\rho_{k} h_{i}\left(x^{k}\right)$ para todo $i \in\{1, \ldots, m\}$ e $\hat{\mu}_{j}^{k}:=\max \left\{0, \mu_{j}^{k}+\rho_{k} g_{j}\left(x^{k}\right)\right\}$ para todo $j \in\{1, \ldots, p\}$. Ainda mais de (AMS07, Teorema 4.1) temos que para $k$ suficientemente grande $\hat{\mu}_{j}^{k}=0$ para todo $j \notin J\left(x^{*}\right)$. Assim, das expressões 4.1.15 e 4.1.16 temos que a condição AKKT2 vale em $x^{*}$.

\section{Método SQP regularizado com convergência a pontos estacionários de segunda ordem.}

Os métodos de Programação Quadrática Sequencial SQP (pelas sua sigla em inglês, Sequencial Quadratic Programming) são uma classe popular de métodos para resolver problemas de programação não linear. Devido às dificuldades teóricas e numéricas associadas à otimização de problemas degenerados ou mal-postos, dois tipos de métodos de programação quadrática seqüencial foram projetados, o método SQP regularizado e o método SQP estabilizado, ver (GR13, IS12). Em (GKR13) Gill, Kungurtsev e Robinson apresentaram uma modificação do método SQP regularizado de (GR13) para obter convergência a pontos que satisfazem a condição WSONC sob a condição MFCQ +WCR. Para informações adicionais ver (Kun13).

Mostraremos que o método proposto em (GKR13) gera sequências que satisfazem a condição sequencial de segunda ordem AKKT2. O problema analisado em (GKR13) é o seguinte

$$
\text { minimizar } f(x) \text { sujeito a } c(x)=0, \quad x \geq 0 .
$$

onde $c: \mathbb{R}^{n} \rightarrow \mathbb{R}^{m}$ e $f: \mathbb{R}^{n} \rightarrow \mathbb{R}$ são funções com derivadas segundas contínuas.

Remark. Para simplificar a verificação, usaremos a mesma notação de (GKR13).

Defina $H(x, \lambda):=\nabla^{2} f(x)-\sum \lambda_{i} \nabla^{2} c_{i}(x)$. Seja $J(x)^{T}$ a matriz cujas linhas são os gradientes $\nabla c_{i}(x)$ para todo $i \in\{1, \ldots, m\}$. Perceba que se definimos as funções $h(x):=-c(x)$ e $g(x):=-x$, a matriz simétrica $H(x, \lambda)$ coincide com a Hessiana da função lagrangiana $L(x, \lambda, \mu)=f(x)+$ $\sum \lambda_{i} h_{i}(x)+\sum \mu_{j} g_{j}(x)$. Defina a função resíduo como $r(x, \lambda):=\|(c(x), \min (x, \nabla f(x)-J(x) \lambda))\|$. Para qualquer ponto viável $x$, o cone crítico fraco é $\mathcal{C}_{A}(x)=\left\{d: J(x)^{T} d=0, d_{j}=0\right.$ para $j \in$ $J(x)\}$ e para um ponto viável fixo $x^{*}$, temos o seguinte cone $\tilde{\mathcal{C}}(x)=\left\{d: J(x)^{T} d=0, d_{j}=\right.$ 0 para $\left.j \in J\left(x^{*}\right)\right\}$. Dados $\gamma, \varepsilon_{a} \in(0,1)$, o conjunto de $\varepsilon$-ativo é definido como $\mathcal{A}_{\varepsilon}(x, \lambda, \mu)=\{i$ : $\left.x_{i} \leq \varepsilon, \operatorname{com} \varepsilon=\min \left(\varepsilon_{a}, \max \left(\mu, r(x, \lambda)^{\gamma}\right)\right)\right\}$. O conjunto $\varepsilon$-livre é definido como $\mathcal{F}_{\varepsilon}(x, \lambda, \mu):=$ $\{1, . ., n\} \backslash \mathcal{A}_{\varepsilon}(x, \lambda, \mu)$. O método proposto em (GKR13) é baseado no método SQP primal-dual de primeira ordem de Gill e Robinson (GR13). A direção de busca é incrementada por uma direção de 
curvatura negativa que facilita a convergência a pontos estacionários de segunda ordem. A direção de curvatura negativa está baseada nas propriedades da seguinte função lagrangiana aumentada

$$
M\left(x, \lambda ; \lambda^{E}, \mu\right)=f(x)-c(x)^{T} \lambda^{E}+\frac{1}{2 \mu}\|c(x)\|^{2}+\frac{\nu}{2 \mu}\left\|c(x)+\mu\left(\lambda-\lambda^{E}\right)\right\|^{2},
$$

onde $\nu \in \mathbb{R}_{+}, \mu$ é um parâmetro de penalidade (positivo) e $\lambda^{E}$ é uma estimativa do multiplicador de Lagrange. Ao igual que (GKR13), denotamos por $B(x, \lambda ; \mu)$ a matriz definida em (GKR13, expressão (2.1)). A matriz $B(x, \lambda ; \mu)$ é uma aproximação da hessiana de $\nabla^{2} M$. A forma exata de $B(x, \lambda ; \mu)$ é dada por (GKR13, expressão $(2.1))$. A matriz $\hat{B}(x, \lambda ; \mu)$ é uma matriz semi-definida positiva, igual a $B(x, \lambda ; \mu)$ quando esta é suficientemente positiva definida, caso contrário, ela tem uma forma específica, ver (GKR13, expressão $(2.3)$ ), que depende da matriz $\hat{H}(x, \lambda)$ de forma que $\hat{H}(x, \lambda)+\mu^{-1} J(x) J(x)^{T}$ é positiva definida, cf. (GR13, Teorema 4.5).

No que resta da discussão, assumimos que $\nu$ é um parâmetro fixo positivo. O algoritmo gera uma sequência $\left\{v^{k}\right\}$ onde $v^{k}=\left(x^{k}, \lambda^{k}\right)$ é a $k$-ésima estimativa da solução primal-dual do problema 4.1.17). Cada iteração pode ser classificada como uma V-, O-, M- ou F- iteração dependendo de certas regras, cf. (GKR13, Algoritmo 3). Pode-se provar que existem infinitas iterações do tipo V-,O- ou M, (GKR13, Teorema 3.2). Experimentos numéricos mostram que o número de iterações do tipo $\mathrm{M}$ ocorre com pouca frequência relativamente ao número total de iterações. O seguinte é um resumo do algoritmo. O algoritmo completo é o algoritmo 3 de (GKR13).

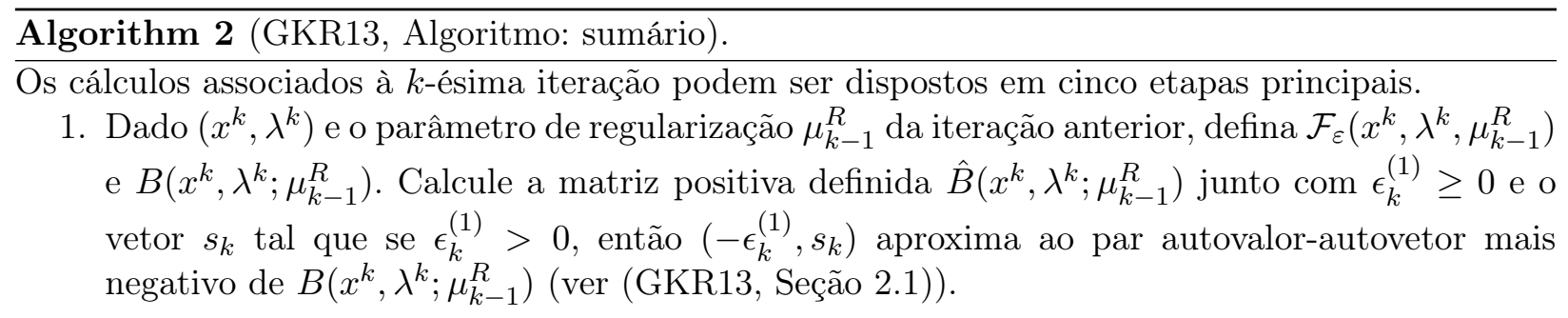

2. Use $\epsilon_{k}^{(1)}$ e $r\left(x^{k}, \lambda^{k}\right)$ para definir os valores de $\lambda^{k E}$ e $\mu_{k}^{R}$ para a $k$-ésima iteração. Ver (GKR13, Seção 2.2).

3. Defina uma direção de descida $d^{k}=\left(p^{k}, q^{k}\right)$ solução do problema convexo associado com a hessiana $B\left(x^{k}, \lambda^{k} ; \mu_{k-1}^{R}\right)$ e o gradiente $\nabla M\left(x^{k}, \lambda^{k} ; \mu_{k}^{R}\right)$. A parte primal de $d^{k}$ satisfaz $x^{k}+p^{k} \geq$ 0. Ver (GKR13, Seção 2.3).

4. Calcule a direção de curvatura negativa $s^{k}=\left(u^{k}, w^{k}\right)$. A parte primal de $s^{k}$ satisfaz $x^{k}+p^{k}+$ $u^{k} \geq 0$. Ver (GKR13, Section 2.3).

5. Faça uma busca linear ao longo do vetor $\triangle v^{k}=s^{k}+d^{k}=\left(p^{k}+u^{k}, q^{k}+w^{k}\right)$ (ver (GKR13, Seção 2.4)). Atualize o parâmetro de penalidade associado à busca linear.

Seguindo (GKR13) , considere as seguintes hipóteses: (i) a sequência de matrizes $\left\{\hat{H}\left(x^{k}, \lambda^{k}\right)\right\}_{k \in \mathbb{N}}$ é uniformente limitada e a sequência dos autovalores mínimos de $\hat{H}\left(x^{k}, \lambda^{k}\right)+\left(1 / \mu_{k}^{R}\right) J\left(x^{k}\right) J\left(x^{k}\right)^{T}$ é uniformente limitada inferiormente e (ii) a sequência $\left\{x^{k}\right\}$ é contida em um conjunto compacto.

Para mostrar que o método gera sequências AKKT2 examinemos a prova do Teorema $3.4 \mathrm{em}$ (GKR13). Seja $\left\{v^{k}=\left(x^{k}, \lambda^{k}\right)\right\}$ a sequência gerada pelo Algoritmo 3 de (GKR13) e suponha que o algoritmo gera infinitos iterados do tipo $\mathrm{V}$ ou O. Seja $x^{*}$ qualquer ponto limite da sequência onde cada iterado é do tipo $\mathrm{V}$ ou do tipo O. Do Algoritmo 3, temos que as quantidades positivas $\phi_{V}^{\max } \mathrm{e}$ $\phi_{O}^{\max }$ são reduzida pela metade durante o processo, ver (GKR13, (2.10)-(2.11)), e assim temos que

$$
\max \left(\left\|c\left(x^{k}\right)\right\|,\left\|\min \left(x^{k}, \nabla f\left(x^{k}\right)-J\left(x^{k}\right) \lambda^{k}\right)\right\|, \epsilon_{k}^{(1)}\right) \rightarrow 0
$$


De 4.1.18 concluímos que $x^{*}$ é viável e de $\left\|\min \left(x^{k}, \nabla f\left(x^{k}\right)-J\left(x^{k}\right) \lambda^{k}\right)\right\| \rightarrow 0$ deduzimos que (4.1.1) da definição de AKKT2 é satisfeita. Agora, procederemos a mostrar que a expressão 4.1.2 da definição de AKKT2 também é satisfeita. Das expressões (GKR13, (3.25)) e (GKR13, (2.6)) temos que

$$
\left\langle v,\left(H\left(x^{k}, \lambda^{k}\right)+\frac{1}{\mu_{k-1}^{R}} J_{k} J_{k}^{T}\right) v\right\rangle \geq-\frac{1}{\theta} \epsilon_{k}^{(1)}\|v\|_{2}^{2} \text { para todo } v \in \mathcal{C}\left(x^{k}\right),
$$

para algum escalar positivo $\theta$ independente de $x^{k}$ e de $\lambda^{k}$. De 4.1.18 temos que $\epsilon_{k}^{(1)} \rightarrow 0$. Usando o Lema 4.0 .7 com $P=H\left(x^{k}, \lambda^{k}\right)+\frac{1}{\mu_{k-1}^{R}} J_{k} J_{k}^{T}+\left(\frac{1}{\theta} \epsilon_{k}^{(1)}+\frac{1}{k}\right) \mathbb{I}$ e $\mathcal{C}$ como $\mathcal{C}\left(x^{k}\right)$, concluímos que

$$
H\left(x^{k}, \lambda^{k}\right)+\frac{1}{\mu_{k-1}^{R}} J_{k} J_{k}^{T}+\left(\frac{1}{\theta} \epsilon_{k}^{(1)}+\frac{1}{k}\right) \mathbb{I}+\sum_{j \in J\left(x^{*}\right)} \theta_{j}^{k} \nabla g_{j} \nabla g_{j}^{T} \succ 0,
$$

para escalares não negativos $\left\{\theta_{j}^{k}: j \in J\left(x^{*}\right)\right\}$ ou equivalentemente

$$
\nabla_{x}^{2} L\left(x^{k}, \lambda^{k}, \mu^{k}\right)+\frac{1}{\mu_{k-1}^{R}} \sum_{i=1}^{m} \nabla h_{i}\left(x^{k}\right) \nabla h_{i}^{T}\left(x^{k}\right)+\sum_{j \in J\left(x^{*}\right)} \theta_{j}^{k} \nabla g_{j} \nabla g_{j}^{T} \succ-\left(\frac{1}{\theta} \epsilon_{k}^{(1)}+\frac{1}{k}\right) \mathbb{I} .
$$

Desde que o lado direito vá para zero quando $k$ tende ao infinito, temos que $x^{*}$ é um ponto AKKT2.

\section{Método de regiões de confiança com convergência para pontos estacionários de segunda ordem}

Mostraremos que o método de região de confiança proposto por Dennis e Vicente (DV97) gera sequências AKKT2. O método proposto em (DV97) é uma extensão do método apresentado em (DEAM97). Considere o seguinte problema de minimização com restrições de igualdade.

$$
\text { minimizar } f(x) \text { sujeito a } C(x)=0 \text {. }
$$

Remark. Para simplificar a analise e a verificação que o método de Dennis e Vicente (DV97) gera sequências AKKT2, usaremos a mesma notação que o artigo (DV97).

Seja $C: \mathbb{R}^{n} \rightarrow \mathbb{R}^{m}(m<n), C=\left(c_{1}, . ., c_{m}\right)^{T}$, onde cada $c_{j}, j \in\{1, \ldots, m\}$, é duas vezes diferenciável. Cada iterado gerado pelo método é denotado por $x^{k}$. Seja $W_{k}$ a matriz cujas colunas formam uma base linear de $\operatorname{Ker} \nabla C\left(x^{k}\right)^{T}$. Seja $H_{k}$ uma aproximação de $\nabla^{2} \ell\left(x^{k}, \mu^{k}\right), \hat{H}_{k}=W_{k}^{T} H_{k} W_{k}$ e $\hat{g}_{k}=W_{k}^{T} \nabla q_{k}\left(s_{k}^{n}\right)$, onde $q_{k}$ é um modelo quadrático da função lagrangiana $\ell(x, \mu)=f(x)+\langle\lambda, h(x)\rangle$ em $\left(x^{k}, \lambda^{k}\right)$ e $s_{k}^{n}$ é chamado de componente quase-normal de $s_{k}$, onde $s_{k}$ é o passo do método. Ver (DV97, 2.Algoritmo e hipóteses gerais).

O método de região de confiança está dado no Algoritmo 3.

Seja $\hat{\Omega}$ um conjunto aberto de $\mathbb{R}^{n}$. Seguindo (DV97), suponha que todas os iterados $x^{k}$ e $x^{k}+s_{k}$ estão em $\hat{\Omega}$ e que as seguintes hipóteses são satisfeitas.

A.1 As funções $f$ e $C$ possuem segunda derivada contínua em $\hat{\Omega}$.

A.2 A matriz $\nabla C(x)$ tem posto completo para todo $x \in \hat{\Omega}$.

A.3 As funções $f, \nabla f, \nabla^{2} f, C, \nabla C$ e $\nabla^{2} c_{i}, i \in\{1, \ldots, m\}$ são limitadas em $\hat{\Omega}$. Além disso a matriz $\left(\nabla C(x)^{T} \nabla C(x)\right)^{-1}$ é limitada uniformemente em $\hat{\Omega}$.

A.4 As sequências $\left\{W_{k}\right\},\left\{H_{k}\right\}$ e $\left\{\lambda_{k}\right\}$ são limitadas.

A.5 A aproximação da Hessiana $H_{k}$ é exata, i.e. $H_{k}=\nabla_{x x}^{2} \ell_{k}$. Além disso, as hessianas $\nabla^{2} f$ e $\nabla^{2} c_{i}$ para todo $i \in\{1, \ldots, m\}$ são Lipschitz contínuas em $\hat{\Omega}$. 


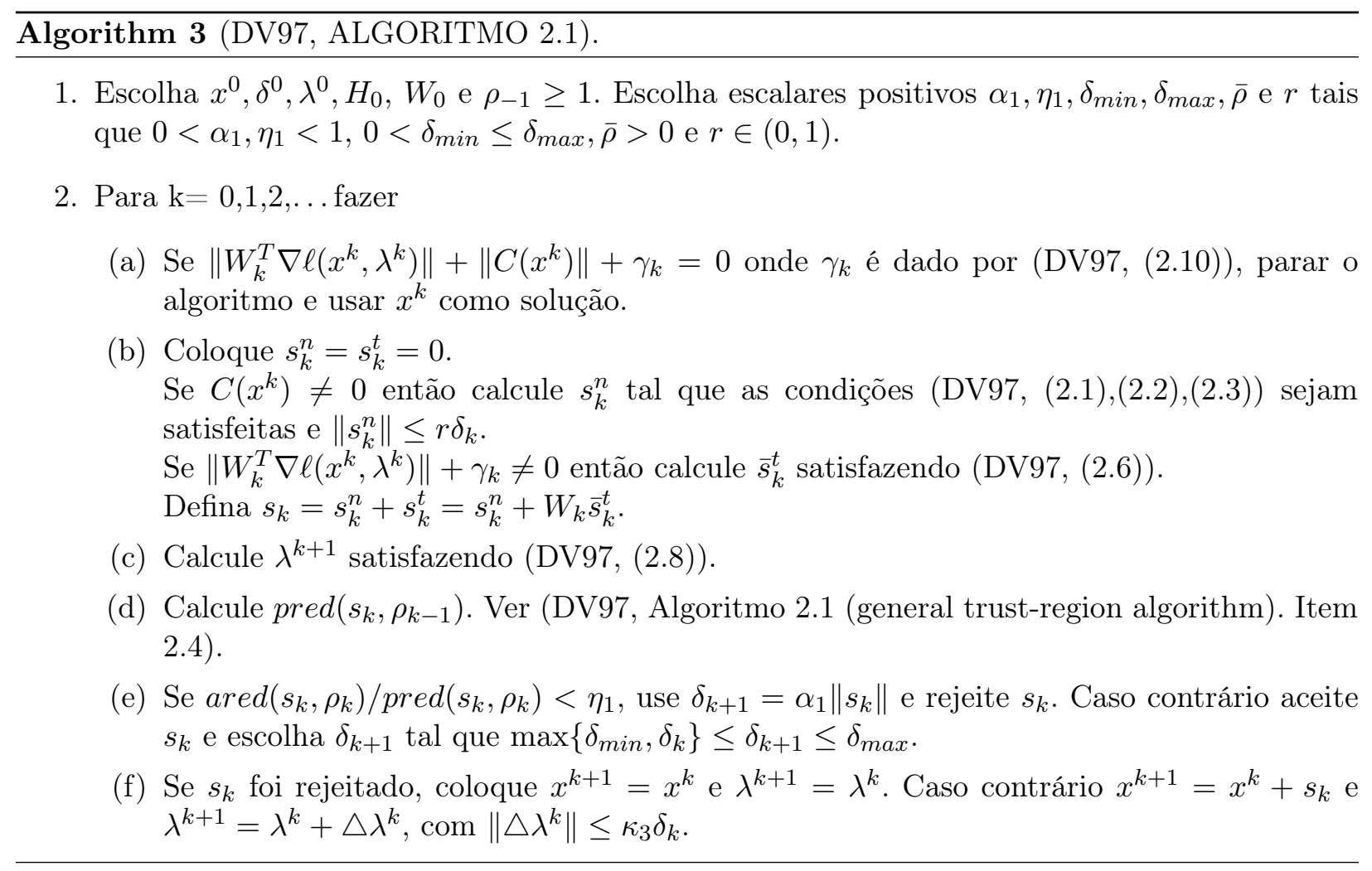

Agora, provaremos que o método gera sequências AKKT2 quando o multiplicador de Lagrange é atualizado de maneira consistente, (DV97, (4.7)).

Primeiro, provaremos que a expressão 4.1.2 da definição de AKKT2 vale para $\left\{\lambda_{k}\right\}$ satisfazendo somente a expressão (DV97, (2.8)). Das condições KKT existe um escalar $\gamma_{k} \geq 0$, (DV97, (2.10)), tal que:

$$
\begin{aligned}
& \hat{H}_{k}+\gamma_{k} W_{k}^{T} W_{k} \text { é semidefinida positiva; } \\
& \left(\hat{H}_{k}+\gamma_{k} W_{k}^{T} W_{k}\right) \bar{s}_{k}=-\bar{g}_{k} ; \\
& \gamma_{k}\left(\bar{\delta}_{k}-\left\|W_{k} \bar{s}_{k}\right\|\right)=0 .
\end{aligned}
$$

Além disso, desde que $\hat{H}_{k}+\gamma_{k} W_{k}^{T} W_{k}=W_{k}^{T}\left(H_{k}+\gamma_{k} \mathbb{I}\right) W_{k}$ é semi-definida positiva e $W_{k}$ é a matriz cuja coluna forma uma base de $\operatorname{Ker} \nabla C\left(x^{k}\right)^{T}$. Do Lema 4.0.7, temos que para todo $k \in \mathbb{N}$, existem sequências $\theta_{i}^{k} \geq 0, i=1, . ., m$ tais que

$$
H_{k}+\sum_{i=1}^{m} \theta_{i}^{k} \nabla c_{i}\left(x^{k}\right) \nabla c_{i}\left(x^{k}\right)^{T}+\left(\gamma_{k}+\frac{1}{k}\right) \mathbb{I} \succ 0 .
$$

De (DV97, Teorema 3.10), a sequência $\left\{x^{k}\right\}$ satisfaz $\liminf \left(\left\|W_{k}^{T} \nabla \ell\left(x^{k}, \lambda^{k}\right)\right\|+\left\|C\left(x^{k}\right)\right\|+\gamma_{k}\right)=0$. Das suposições temos que $\left\{x^{k}\right\}$ é uma sequência limitada. Depois de tomar uma subsequência adequada (se fosse necessário) de $\left\{x^{k}\right\}$, podemos supor que $x^{k} \rightarrow x^{*}$ para algum $x^{*} \in \mathbb{R}^{n}$,

$$
\gamma_{k} \rightarrow 0, \quad\left\|C\left(x^{k}\right)\right\| \rightarrow 0 \quad \text { e } \quad\left\|W_{k}^{T} \nabla_{x} \ell\left(x^{k}, \lambda^{k}\right)\right\| \rightarrow 0 .
$$

Deste modo, como $\gamma_{k} \rightarrow 0$, deduzimos de 4.1.20 que a expressão 4.1.2 da definição de AKKT2 vale. Para provar que 4.1.1) da definição de AKKT2 também é satisfeita, escolha os multiplicadores de Lagrange $\lambda^{k}$ como $-\left(\nabla C\left(x^{k}\right)^{T} \nabla C\left(x^{k}\right)\right)^{-1} \nabla C_{k}^{T} \nabla f\left(x^{k}\right)$, ver (DV97, Lema 4.2). Para cada $k \in \mathbb{N}$, decomponha $\nabla_{x} \ell\left(x^{k}, \lambda^{k}\right)$ como:

$$
\nabla_{x} \ell\left(x^{k}, \lambda^{k}\right)=W_{k} u^{k}+\nabla C\left(x^{k}\right) v^{k},
$$


onde $W_{k} u^{k} \in \operatorname{Ker}\left(\nabla C\left(x^{k}\right)^{T}\right)$ e $\nabla C\left(x^{k}\right) v^{k} \in \operatorname{Ker}\left(\nabla C\left(x^{k}\right)^{T}\right)^{\perp}=\operatorname{Im}\left(\nabla C\left(x^{k}\right)\right)$ para certos $u^{k}$ e $v^{k}$. Multiplicando 4.1.22 por $\left(u^{k}\right)^{T} W_{k}^{T}$ e usando $\lim \left\|W_{k}^{T} \nabla_{x} \ell\left(x^{k}, \lambda^{k}\right)\right\|=0$ temos que $W_{k} u^{k} \rightarrow 0$. Multiplicando 4.1.22 por $\nabla C\left(x^{k}\right)^{T}$ e usando a existência da matriz inversa $\left(\nabla C\left(x^{k}\right)^{T} \nabla C\left(x^{k}\right)\right)^{-1}$, obtemos $v^{k}=\left(\nabla C(x)^{T} \nabla C(x)\right)^{-1} \nabla C_{k}^{T} \nabla f\left(x^{k}\right)+\lambda^{k}=0$. Portanto, da expressão 4.1.22, temos que $\nabla_{x} \ell\left(x^{k}, \lambda^{k}\right)=W_{k} u^{k} \rightarrow 0$. Finalmente, de 4.1.21), segue que $\left\|C\left(x^{k}\right)\right\| \rightarrow 0$ e que $x^{*}$ é viável. Assim, $x^{*}$ é um ponto AKKT2, como queríamos provar.

\subsection{Nova CQ associada com a condição AKKT2}

A análise da convergência global para pontos estacionários de segunda ordem do método de lagrangiano aumentado (AMS07) e do método SQP regularizado (GKR13) está baseada na condição conjunta MFCQ e WCR. Desde que ambos métodos geram sequências AKKT2, a pergunta natural é se a Proposição 4.1.4 pode ser demonstrada usando condições de qualificação mais fracas. Como veremos nesta seção, a resposta é afirmativa. Isto implica uma melhora na teoria da convergência global de cada algoritmo que gera sequências AKKT2. Ainda mais nesta seção, forneceremos a condição de qualificação mínima para a qual todo ponto AKKT2 satisfaz WSONC.

Defina, para cada $x \in \mathbb{R}^{n}$, o cone

$$
C^{\mathcal{W}}\left(x, x^{*}\right):=\left\{d \in \mathbb{R}^{n}:\left\langle\nabla h_{i}(x), d\right\rangle=0 \text { para } i \in\{1, \ldots, m\} ;\left\langle\nabla g_{j}(x), d\right\rangle=0 \text { para } j \in J\left(x^{*}\right)\right\} .
$$

O cone $C^{\mathcal{W}}\left(x, x^{*}\right)$ é uma perturbação do cone crítico fraco $C^{\mathcal{W}}\left(x^{*}\right)$ ao redor do ponto viável $x^{*} \in \Omega$. Claramente, $C^{\mathcal{W}}\left(x, x^{*}\right)$ é um subespaço e $C^{\mathcal{W}}\left(x^{*}, x^{*}\right)$ coincide com o cone crítico fraco $C^{\mathcal{W}}\left(x^{*}\right)$. Usando a linguagem da análise variacional, podemos reescrever o Lema 4.1.3 como: a condição WCR implica a semi-continuidade interior da multifunção $C^{\mathcal{W}}\left(x, x^{*}\right)$ em $x=x^{*}$, isto é, $C^{\mathcal{W}}\left(x^{*}, x^{*}\right) \subset \liminf _{x \rightarrow x^{*}} C^{\mathcal{W}}\left(x, x^{*}\right)$. De fato, a semi-continuidade interior de $C^{\mathcal{W}}\left(x, x^{*}\right)$ em $x=x^{*}$ é equivalente à condição WCR. Ver Proposição A.0.2.

Agora vamos definir o objeto principal desta seção. Para cada $x \in \mathbb{R}^{n}$ considere o seguinte cone

$$
K_{2}^{\mathcal{W}}(x):=\bigcup_{\substack{(\lambda, \mu) \in \mathbb{R}^{m} \times \mathbb{R}_{+}^{p}, \mu_{j}=0 \text { para } j \notin J\left(x^{*}\right)}}\left\{\begin{array}{l}
\left(\sum_{i=1}^{m} \lambda_{i} \nabla h_{i}(x)+\sum_{j \in J\left(x^{*}\right)} \mu_{j} \nabla g_{j}(x), H\right), \text { tal que } \\
H \preceq \sum_{i=1}^{m} \lambda_{i} \nabla^{2} h_{i}(x)+\sum_{j \in J\left(x^{*}\right)} \mu_{j} \nabla^{2} g_{j}(x) \text { sobre } C^{W}\left(x, x^{*}\right)
\end{array}\right\} .
$$

O conjunto $K_{2}^{\mathcal{W}}(x)$ é um cone convexo incluso em $\mathbb{R}^{n} \times \operatorname{Sym}(\mathrm{n})$ que nos permite escrever a condição fraca de segunda ordem WSONC em uma forma mais compacta, isto é,

$$
\left(-\nabla f\left(x^{*}\right),-\nabla^{2} f\left(x^{*}\right)\right) \in K_{2}^{\mathcal{W}}\left(x^{*}\right) .
$$

A seguinte definição é a nossa nova condição de qualificação associada à condição sequencial AKKT2.

Definição 4.2.1. Dizemos que $x^{*} \in \Omega$ satisfaz a Propriedade de Continuidade do Cone de Segunda Ordem (CCP2) se a multifunção $x \mapsto K_{2}^{\mathcal{W}}(x)$, definida em 4.2.2), é semicontinua exteriormente em $x^{*}$, isto é,

$$
\limsup _{x \rightarrow x^{*}} K_{2}^{\mathcal{W}}(x) \subset K_{2}^{\mathcal{W}}\left(x^{*}\right) .
$$

Como mostra o seguinte teorema, a condição CCP2 é a condição de qualificação mais fraca que pode ser usada para generalizar a Proposição 4.1.4.

Teorema 4.2.1. Seja $x^{*} \in \Omega$ um ponto viável. As seguintes condições são equivalentes:

(1) CCP2 vale em $x^{*}$;

(2) Para toda função objetivo $f: \mathbb{R}^{n} \rightarrow \mathbb{R}$ do problema (2.0.3) tal que a condição AKKT2 vale em $x^{*}$, a condição WSONC é satisfeita em $x^{*}$. 
Demonstração. Primeiro, suponha que a condição CCP2 vale em $x^{*}$ e existe uma função objetivo $f$ tal que a condição AKKT2 é satisfeita. Da definição AKKT2, existem sequências $\left\{x^{k}\right\},\left\{\lambda^{k}\right\},\left\{\mu^{k}\right\}$, $\left\{\eta^{k}\right\},\left\{\theta^{k}\right\},\left\{\delta_{k}\right\}$, com $\mu^{k} \geq 0, \mu_{j}^{k}=0$ para $j \notin J\left(x^{*}\right)$ e $\theta^{k} \geq 0, \theta_{j}^{k}=0$ para $j \notin J\left(x^{*}\right)$ tais que $x^{k} \rightarrow x^{*}, \delta_{k} \rightarrow 0$ e

a) $\varepsilon^{k}:=\nabla f\left(x^{k}\right)+\sum_{i=1}^{m} \lambda_{i}^{k} \nabla h_{i}\left(x^{k}\right)+\sum_{j \in J\left(x^{*}\right)} \mu_{j}^{k} \nabla g_{j}\left(x^{k}\right) \rightarrow 0 ;$

b) $\nabla_{x}^{2} L\left(x^{k}, \lambda^{k}, \mu^{k}\right)+\sum_{i=1}^{m} \eta_{i}^{k} \nabla h_{i}\left(x^{k}\right) \nabla h_{i}\left(x^{k}\right)^{T}+\sum_{j \in J\left(x^{*}\right)} \theta_{j}^{k} \nabla g_{j}\left(x^{k}\right) \nabla g_{j}\left(x^{k}\right)^{T} \succeq-\delta_{k} \mathbb{I}$.

Usando o item (a) e o item (b), deduzimos que $\left(-\nabla f\left(x^{k}\right)+\varepsilon^{k},-\nabla^{2} f\left(x^{k}\right)-\delta_{k} \mathbb{I}\right) \in K_{2}^{\mathcal{W}}\left(x^{k}\right)$. Usando, agora, a continuidade de $\nabla f(x)$ e de $\nabla^{2} f(x)$ junto com a semi-continuidade exterior de $K_{2}^{\mathcal{W}}(x)$ em $x^{*}$, obtemos que $\left(-\nabla f\left(x^{*}\right),-\nabla^{2} f\left(x^{*}\right)\right) \in K_{2}^{\mathcal{W}}\left(x^{*}\right)$ e como consequência WSONC vale.

Provemos a outra implicação. Seja $(w, W)$ um elemento de $\lim \sup K_{2}^{\mathcal{W}}(x)$ quando $x \rightarrow x^{*}$. Mostraremos que $(w, W)$ pertence a $K_{2}^{\mathcal{W}}\left(x^{*}\right)$. De definição de limite exterior, existem sequências $\left\{x^{k}\right\},\left\{\lambda_{i}^{k}\right\},\left\{\mu_{j}^{k}\right\}$ com $\mu_{j}^{k}=0$ para $j \notin J\left(x^{*}\right)$ e $\left\{H^{k}\right\}$ tais que $x^{k} \rightarrow x^{*}$,

$$
\left(\sum_{i=1}^{m} \lambda_{i}^{k} \nabla h_{i}\left(x^{k}\right)+\sum_{j \in J\left(x^{*}\right)} \mu_{j}^{k} g_{j}\left(x^{k}\right), H^{k}\right) \rightarrow(w, W)
$$

$\mathrm{e}$

$$
H^{k} \preceq \sum_{i=1}^{m} \lambda_{i}^{k} \nabla^{2} h_{i}\left(x^{k}\right)+\sum_{j \in J\left(x^{*}\right)} \mu_{j}^{k} \nabla^{2} g_{j}\left(x^{k}\right) \text { sobre } C^{\mathcal{W}}\left(x^{k}, x^{*}\right) .
$$

Defina a função

$$
f(x):=-\left\langle w, x-x^{*}\right\rangle-\frac{1}{2} W\left(x-x^{*}, x-x^{*}\right) .
$$

Mostraremos que a condição AKKT2 vale em $x^{*} \operatorname{com} f(x)$ como função objetivo. Do cálculo temos que $\nabla f(x)=-w-W\left(x-x^{*}\right)$ e $\nabla^{2} f(x)=-W$. Para provar que 4.1.1 vale, é suficiente notar que

$$
\lim _{k \rightarrow \infty} \nabla_{x} L\left(x^{k}, \lambda^{k}, \mu^{k}\right)=\lim _{k \rightarrow \infty}\left(\sum_{i=1}^{m} \lambda_{i}^{k} \nabla h_{i}\left(x^{k}\right)+\sum_{j \in J\left(x^{*}\right)} \mu_{j}^{k} g_{j}\left(x^{k}\right)-w\right)-\lim _{k \rightarrow \infty} W\left(x^{k}-x^{*}\right)=0 .
$$

Agora, para provar a validade de 4.1.2, usamos o Lema 4.0.7 com

$$
P^{k}:=\sum_{i=1}^{m} \lambda_{i}^{k} \nabla^{2} h_{i}\left(x^{k}\right)+\sum_{j \in J\left(x^{*}\right)} \mu_{j}^{k} \nabla^{2} g_{j}\left(x^{k}\right)-H^{k}+\frac{1}{k} \mathbb{I},
$$

e $a_{i}$ como as colunas da matriz $\left[\nabla h_{i}\left(x^{k}\right), i=1, \ldots, m ; \nabla g_{j}\left(x^{k}\right), j \in J\left(x^{*}\right)\right]$. Assim, pelo Lema 4.0.7 existem sequências positivas $\left\{\theta^{k}\right\}$ e $\left\{\eta^{k}\right\}$ tais que:

$$
S^{k}:=P^{k}+\sum_{i=1}^{m} \eta_{i}^{k} \nabla h_{i}\left(x^{k}\right) \nabla h_{i}\left(x^{k}\right)^{T}+\sum_{j \in J\left(x^{*}\right)} \theta_{j}^{k} \nabla g_{j}\left(x^{k}\right) \nabla g_{j}\left(x^{k}\right)^{T} \succ 0 .
$$

Coloque $\theta_{j}^{k}=0$ para $j \notin J\left(x^{*}\right)$. Usando 4.2.5, 4.2 .6 e $\nabla^{2} f(x)=-W$, obtemos que

$$
\nabla_{x}^{2} L\left(x^{k}, \lambda^{k}, \mu^{k}\right)+\sum_{i=1}^{m} \eta_{i}^{k} \nabla h_{i}\left(x^{k}\right) \nabla h_{i}\left(x^{k}\right)^{T}+\sum_{j \in J\left(x^{*}\right)} \theta_{j}^{k} \nabla g_{j}\left(x^{k}\right) \nabla g_{j}\left(x^{k}\right)^{T}=-W+H^{k}+S^{k}-\frac{1}{k} \mathbb{I} .
$$

Agora, procederemos a achar um limitante inferior para a matriz simétrica do lado direito da expressão (4.2.7). Usando a relação de Rayleigh

$$
-W+H^{k} \succeq-\left|\lambda_{1}\left(W-H^{k}\right)\right| \mathbb{I},
$$


onde $\lambda_{1}\left(W-H^{k}\right)$ denota o menor auto-valor de $W-H^{k}$. De 4.2.6), $S^{k} \succ 0$, assim temos que

$$
-W+H^{k}+S^{k}-\frac{1}{k} \mathbb{I} \succeq-\left|\lambda_{1}\left(W-H^{k}\right)\right| \mathbb{I}+S^{k}-\frac{1}{k} \mathbb{I} \succeq-\left|\lambda_{1}\left(W-H^{k}\right)\right| \mathbb{I}-\frac{1}{k} \mathbb{I}=-\delta_{k} \mathbb{I},
$$

onde $\delta_{k}:=\left|\lambda_{1}\left(W-H^{k}\right)\right|+1 / k$. Como $H^{k} \rightarrow W$ e $\delta_{k} \rightarrow 0$, substituindo 4.2.8) em 4.2.7), temos que a condição 4.1.2 vale. Assim, $x^{*}$ é um ponto AKKT2 e pela hipótese, WSONC vale em $x^{*}$. Usando 4.2.3), $(w, W)=\left(-\nabla f\left(x^{*}\right),-\nabla^{2} f\left(x^{*}\right)\right)$ pertence a $K_{2}^{\mathcal{W}}\left(x^{*}\right)$, concluindo com a demonstração.

Como AKKT2 é uma condição de otimalidade, pelo Teorema 4.2.1, temos o seguinte resultado.

Corolário 4.2.2. Se $x^{*}$ é um minimizador local de 2.0.3) tal que CCP2 vale em $x^{*}$, então WSONC vale em $x^{*}$.

\subsection{Relações com as CQs conhecidas}

\subsubsection{Relação com a condição de qualificação conjunta MFCQ+WCR}

Direitamente da Proposição 4.1 .4 e do Teorema 4.2.1 temos que o resultado a seguir.

Proposição 4.3.1. A condição CCP2 é implicada por $M F C Q+W C R$.

De fato, a condição CPP2 é estritamente mais fraca que MFCQ +WCR, como o seguinte exemplo mostra.

Exemplo 4.3.1. (A condição CCP2 não implica $M F C Q+W C R$.)

Em $\mathbb{R}$, considere $x^{*}=0$ e as restrições de desigualdade definidas pelas funções

$$
g_{1}(x)=x \quad \text { e } \quad g_{2}(x)=-x .
$$

Em $x^{*}$, temos que CPP2 vale mas MFCQ falha (como consequência MFCQ+WCR falha). Para ver isso, notemos que desde que os gradientes das funções $g_{1}$ e $g_{2}$ são positivamente dependente, MFCQ não vale em $x^{*}=0$. Calculemos o cone $K_{2}^{\mathcal{W}}(x)$ para todo $x \in \mathbb{R}$. Como $\nabla g_{1}(x)=1, \nabla^{2} g_{1}(x)=$ $0, \nabla g_{2}(x)=-1$ e $\nabla^{2} g_{2}(x)=0$ temos que $C^{\mathcal{W}}\left(x, x^{*}\right)=0$. Desta forma, qualquer matriz $H \in$ $\operatorname{Sym}(1)=\mathbb{R}$ satisfaz a desigualdade matricial:

$$
H \preceq \mu_{1} \nabla^{2} g_{1}(x)+\mu_{2} \nabla^{2} g_{2}(x)=0 \text { sobre } C^{\mathcal{W}}\left(x, x^{*}\right)=\{0\} \text { para todo } \mu_{1}, \mu_{2} \geq 0 .
$$

Por conseguinte, $K_{2}^{\mathcal{W}}(x)=\mathbb{R} \times \mathbb{R}$ para todo $x \in \mathbb{R}$ e $K_{2}^{\mathcal{W}}(x)$ é semicontínua exteriormente em $\mathbb{R}$.

\subsubsection{Relação com a condição de qualificação RCRCQ}

Nesta subseção provaremos que RCRCQ é estritamente mais forte que a condição CPP2. Para continuar, precisaremos dos seguintes lemas preparatórios. O primeiro lema é consequência de um resultado clássico de análise: o Teorema do posto constante, cf. (Spi65, Teorema 13.2).

Lema 4.3.2. Sejam $\mathcal{I}$ e $\mathcal{J}$ dois conjuntos de indices. Suponha que os gradientes $\left\{\nabla h_{i}(z), \nabla g_{j}(z)\right.$ : $i \in \mathcal{I}, j \in \mathcal{J}\}$ têm posto constante em uma vizinhança de $x \in \mathbb{R}^{n}$. Então, para cada $d \in \mathbb{R}^{n}$ tal que

$$
\left\langle\nabla h_{i}(x), d\right\rangle=0 \quad \text { para } i \in \mathcal{I} \text { e }\left\langle\nabla g_{j}(x), d\right\rangle=0 \quad \text { para } j \in \mathcal{J}
$$

existe uma curva $t \rightarrow \phi(t), t \in(-T, T), T>0$ duas vezes diferenciável tal que $\phi(0)=x, \phi^{\prime}(0)=d$ e para todo $i \in \mathcal{I}$ e $j \in \mathcal{J}$ temos que $h_{i}(\phi(t))=h_{i}(x)$ e $g_{j}(\phi(t))=g_{j}(x) \forall t \in(-T, T)$.

O seguinte lema é uma variação do Lema de Caratheódory útil para a condição RCRCQ. 
Lema 4.3.3. AHSS12a, Lema 1) Suponha que $v=\sum_{i \in \mathcal{I}} \alpha_{i} p_{i}+\sum_{j \in \mathcal{J}} \beta_{j} q_{j}$ com $p_{i}, q_{j} \in \mathbb{R}^{n}$ para todo $i \in \mathcal{I}, j \in \mathcal{J},\left\{p_{i}\right\}_{i \in \mathcal{I}}$ é um conjunto linearmente independente e $\alpha_{i}, \beta_{j}$ são diferentes de zero para todo $i \in \mathcal{I}, j \in \mathcal{J}$. Então existe um subconjunto $\mathcal{J}^{\prime} \subset \mathcal{J}$ e escalares $\hat{\alpha}_{i}, \hat{\beta}_{j}$ para todo $i \in \mathcal{I}$, $j \in \mathcal{J}^{\prime}$ tais que

- $v=\sum_{i \in \mathcal{I}} \hat{\alpha}_{i} p_{i}+\sum_{j \in \mathcal{J}^{\prime}} \hat{\beta}_{j} q_{j}$

- Para todo $j \in \mathcal{J}^{\prime}$ temos $\beta_{j} \hat{\beta}_{j}>0$;

- $\left\{p_{i}, q_{j}\right\}_{i \in \mathcal{I}, j \in \mathcal{J}^{\prime}}$ é um conjunto linearmente independente.

Uma caracterização útil de RCRCQ é feita a seguir.

Teorema 4.3.4. AHSS12a, Teorema 1) Seja $I \subset\{1, \ldots m\}$ um conjunto de índices tal que $\left\{\nabla h_{i}(x): i \in I\right\}$ é uma base linear de $\operatorname{span}\left\{\nabla h_{i}(x): i \in\{1, \ldots, m\}\right\}$. Um ponto viável $x \in \Omega$ satisfaz RCRCQ se, e somente se, existe uma vizinhança $V$ de $x$ tal que

a) $\left\{\nabla h_{i}(y): i \in\{1, \ldots, m\}\right\}$ tem o mesmo posto para todo $y \in V$;

b) Para cada $J \subset A(x)$, se $\left\{\nabla h_{i}(x), \nabla g_{j}(x): i \in\{1, \ldots, m\}, j \in J\right\}$ é linearmente dependente então $\left\{\nabla h_{i}(y), \nabla g_{j}(y): i \in\{1, \ldots, m\}, j \in J\right\}$ é linearmente dependente para todo $y \in V$.

A próxima proposição estabelece a primeira relação entre a CCP2 e a RCRCQ.

Proposição 4.3.5. A condição CCP2 é implicada pela $R C R C Q$.

Demonstração. Seja $(w, W)$ um elemento de $\lim \sup _{x \rightarrow x^{*}} K_{2}^{\mathcal{W}}(x)$. Pela definição de limite exterior, existem sequências $\left\{x^{k}\right\},\left\{\lambda_{i}^{k}\right\},\left\{\mu_{j}^{k}\right\}$ com $\mu_{j}^{k}=0$ para $j \notin J\left(x^{*}\right)$ e $\left\{H^{k}\right\}$ tais que $x^{k} \rightarrow x^{*}$,

$$
w^{k}:=\sum_{i=1}^{m} \lambda_{i}^{k} \nabla h_{i}\left(x^{k}\right)+\sum_{j \in J\left(x^{*}\right)} \mu_{j}^{k} \nabla g_{j}\left(x^{k}\right) \rightarrow w \text { e } H^{k} \rightarrow W,
$$

onde $H^{k} \preceq \sum_{i=1}^{m} \lambda_{i}^{k} \nabla^{2} h_{i}\left(x^{k}\right)+\sum_{j \in J\left(x^{*}\right)} \mu_{j}^{k} \nabla^{2} g_{j}\left(x^{k}\right)$ sobre $C^{\mathcal{W}}\left(x^{k}, x^{*}\right)$.

Seja $\mathcal{I} \subset\{1, \ldots, m\}$ um subconjunto de índices tal que os gradientes $\left\{\nabla h_{i}\left(x^{*}\right)\right\}_{i \in \mathcal{I}}$ formem uma base linear do subespaço linear gerado por $\left\{\nabla h_{i}\left(x^{*}\right): i \in\{1, \ldots, m\}\right\}$. Da continuidade, $\left\{\nabla h_{i}\left(x^{k}\right): i \in\right.$ $\mathcal{I}\}$ é linearmente independente para $k$ suficientemente grande. Pelo Teorema 4.3.4, item (a), temos que $\left\{\nabla h_{i}\left(x^{k}\right): i \in \mathcal{I}\right\}$ é uma base do subespaço linear gerado por $\left\{\nabla h_{i}\left(x^{k}\right): i \in\{1, \ldots, m\}\right\}$ para $k$ suficientemente grande. Então, existe uma sequência $\bar{\lambda}_{i}^{k} \in \mathbb{R}$ para $i \in \mathcal{I}$ tal que $\sum_{i=1}^{m} \lambda_{i}^{k} \nabla h_{i}\left(x^{k}\right)=$ $\sum_{i \in \mathcal{I}} \bar{\lambda}_{i}^{k} \nabla h_{i}\left(x^{k}\right)$. Assim, podemos escrever:

$$
w^{k}=\sum_{i \in \mathcal{I}} \bar{\lambda}_{i}^{k} \nabla h_{i}\left(x^{k}\right)+\sum_{j \in J\left(x^{*}\right)} \mu_{j}^{k} \nabla g_{j}\left(x^{k}\right) .
$$

Usando o Lema 4.3 .3 para a expressão acima, encontramos um subconjunto de índices $\mathcal{J}_{k} \subset$ $J\left(x^{*}\right)$ e multiplicadores $\hat{\lambda}_{i}^{k} \in \mathbb{R}, i \in \mathcal{I}$ e $\hat{\mu}_{j}^{k} \in \mathbb{R}_{+}, j \in \mathcal{J}_{k}$ para $k$ suficientemente grande tal que

$$
w^{k}=\sum_{i \in \mathcal{I}} \hat{\lambda}_{i}^{k} \nabla h_{i}\left(x^{k}\right)+\sum_{j \in \mathcal{J}_{k}} \hat{\mu}_{j}^{k} \nabla g_{j}\left(x^{k}\right)
$$

e $\left\{\nabla h_{i}\left(x^{k}\right), \nabla g_{j}\left(x^{k}\right): i \in \mathcal{I}, j \in \mathcal{J}_{k}\right\}$ é um conjunto linearmente independente. Como $A\left(x^{*}\right)$ é um conjunto de índices finitos, sem perda de generalidade, podemos supor que $\mathcal{J}:=\mathcal{J}_{k}$ para todo $k$ suficientemente grande. Do Teorema 4.3.4 item (b), temos que $\left\{\nabla h_{i}\left(x^{*}\right), \nabla g_{j}\left(x^{*}\right): i \in \mathcal{I}, j \in \mathcal{J}\right\}$ é um conjunto linearmente independente e $\left\{\hat{\lambda}_{i}^{k}, \hat{\mu}_{j}^{k}: i \in \mathcal{I}, j \in \mathcal{J}\right\}_{k \in \mathbb{N}}$ é uma sequência limitada, assim sem perda de generalidade podemos supor que $\hat{\lambda}_{i}^{k} \rightarrow \lambda_{i}$ e $\hat{\mu}_{j}^{k} \rightarrow \mu_{j}$. Tomando limites em 4.3.5 obtemos que $w=\sum_{i \in \mathcal{I}} \lambda_{i} \nabla h_{i}\left(x^{*}\right)+\sum_{j \in \mathcal{J}} \mu_{j} \nabla g_{j}\left(x^{*}\right)$. Para cada $k \in \mathbb{N}$, defina $\hat{\lambda}_{i}^{k}=0$ para 
$i \notin \mathcal{I}$ e $\hat{\mu}_{j}^{k}=0$ para $j \notin \mathcal{J}$, defina também $\lambda_{i}=0$ para $i \notin \mathcal{I}$ e $\mu_{j}=0$ para $j \notin \mathcal{J}$. Agora, mostraremos que $d \in C^{\mathcal{W}}\left(x^{*}\right)$ se cumpre $\left.H(d, d)\right\rangle \leq \sum_{i=1}^{m} \lambda_{i} \nabla^{2} h_{i}\left(x^{*}\right)(d, d)+\sum_{j \in J\left(x^{*}\right)} \mu_{j} \nabla^{2} g_{j}\left(x^{*}\right)(d, d)$.

Defina para cada $k \in \mathbb{N}, \Lambda_{i}^{k}=\lambda_{i}^{k}-\hat{\lambda}_{i}^{k}, \Upsilon_{j}^{k}=\mu_{j}^{k}-\hat{\mu}_{j}^{k}$. De 4.3.5 e 4.3.3 temos que

$$
\sum_{i=1}^{m} \Lambda_{i}^{k} \nabla h_{i}\left(x^{k}\right)+\sum_{j \in J\left(x^{*}\right)} \Upsilon_{j}^{k} \nabla g_{j}\left(x^{k}\right)=0 \quad \text { para } k \in \mathbb{N} \text { suficientemente grande. }
$$

Seja $d \in C^{\mathcal{W}}\left(x^{*}\right)$. Uma vez que RCRCQ implica WCR, temos que existe uma sequência $d^{k} \rightarrow d$ tal que $d^{k} \in C^{\mathcal{W}}\left(x^{k}, x^{*}\right)$, ver Lema 4.1 .3 , portanto

$$
\begin{aligned}
H^{k}\left(d^{k}, d^{k}\right) & \leq \sum_{i=1}^{m} \lambda_{i}^{k} \nabla^{2} h_{i}\left(x^{k}\right)\left(d^{k}, d^{k}\right)+\sum_{j \in J\left(x^{*}\right)} \mu_{j}^{k} \nabla^{2} g_{j}\left(x^{k}\right)\left(d^{k}, d^{k}\right) \\
& \leq \sum_{i=1}^{m} \hat{\lambda}_{i}^{k} \nabla^{2} h_{i}\left(x^{k}\right)\left(d^{k}, d^{k}\right)+\sum_{j \in J\left(x^{*}\right)} \hat{\mu}_{j}^{k} \nabla^{2} g_{j}\left(x^{k}\right)\left(d^{k}, d^{k}\right)+\Xi^{k}
\end{aligned}
$$

em que

$$
\Xi^{k}:=\sum_{i=1}^{m} \Lambda_{i}^{k} \nabla^{2} h_{i}\left(x^{k}\right)\left(d^{k}, d^{k}\right)+\sum_{j \in J\left(x^{*}\right)} \Upsilon_{j}^{k} \nabla^{2} g_{j}\left(x^{k}\right)\left(d^{k}, d^{k}\right) .
$$

De RCRCQ, temos que para $k$ suficientemente grande, existe uma vizinhança de $x^{k}$, na qual o posto $\left\{\nabla h_{i}(x), \nabla g_{j}(x): i \in\{1, \ldots, m\}, j \in J\left(x^{*}\right)\right\}$ é constante, assim pelo Lema 4.3.2, existe uma curva $t \rightarrow \phi_{k}(t)$ para $t \in\left(-T_{k}, T_{k}\right), T_{k}>0$ com $\phi_{k}(0)=x^{k}, \phi_{k}^{\prime}(0)=d^{k}$ tal que $h_{i}\left(\phi_{k}(t)\right)=h_{i}\left(x^{k}\right)$ para todo $i \in\{1, \ldots, m\}$ e $g_{j}\left(\phi_{k}(t)\right)=g_{j}\left(x^{k}\right)$ para todo $j \in J\left(x^{*}\right)$. Denote $v^{k}:=\phi_{k}^{\prime \prime}(0)$ Derivando $h_{i}\left(\phi_{k}(t)\right)=0, i \in\{1, \ldots, m\}$ e $g_{j}\left(\phi_{k}(t)\right)=0, j \in J\left(x^{*}\right)$ duas vezes em $t=0$, obtemos:

$$
\begin{aligned}
& \left\langle\nabla h_{i}\left(x^{k}\right), v^{k}\right\rangle+\nabla^{2} h_{i}\left(x^{k}\right)\left(d^{k}, d^{k}\right)=0, \text { para todo } i \in\{1, \ldots, m\} ; \\
& \left\langle\nabla g_{j}\left(x^{k}\right), v^{k}\right\rangle+\nabla^{2} g_{j}\left(x^{k}\right)\left(d^{k}, d^{k}\right)=0, \text { para todo } j \in J\left(x^{*}\right) .
\end{aligned}
$$

Assim, substituindo as expressões 4.3.10 e 4.3.11 em 4.3.9

$$
\begin{aligned}
\Xi^{k} & =-\sum_{i=1}^{m} \Lambda_{i}^{k}\left\langle\nabla h_{i}\left(x^{k}\right), v^{k}\right\rangle-\sum_{j \in J\left(x^{*}\right)} \Upsilon_{j}^{k}\left\langle\nabla g_{j}\left(x^{k}\right), v^{k}\right\rangle \\
& =-\left\langle\sum_{i=1}^{m} \Lambda_{i}^{k} \nabla h_{i}\left(x^{k}\right)+\sum_{j \in J\left(x^{*}\right)} \Upsilon_{j}^{k} \nabla g_{j}\left(x^{k}\right), v^{k}\right\rangle=0,
\end{aligned}
$$

onde na última igualdade usamos 4.3.6. Como $\Xi^{k}=0$ para todo $k$ suficientemente grande, temos que (4.3.8) torna-se

$$
H^{k}\left(d^{k}, d^{k}\right) \leq \sum_{i=1}^{m} \hat{\lambda}_{i}^{k} \nabla^{2} h_{i}\left(x^{k}\right)\left(d^{k}, d^{k}\right)+\sum_{j \in J\left(x^{*}\right)} \hat{\mu}_{j}^{k} \nabla^{2} g_{j}\left(x^{k}\right)\left(d^{k}, d^{k}\right) .
$$

Tomando limite em 4.3.14, concluímos com a demonstração.

Como consequência, CRCQ também implica CCP2.

Exemplo 4.3.2. A condição CCP2 é estritamente mais fraca que $R C R C Q$. 
Em $\mathbb{R}^{2}$, considere $x^{*}=(0,0)^{\top}$ e o seguinte conjunto de restrições definidas pelas funções

$$
\begin{aligned}
& h_{1}\left(x_{1}, x_{2}\right)=x_{1} ; \\
& g_{1}\left(x_{1}, x_{2}\right)=-x_{1}^{2}+x_{2} ; \\
& g_{2}\left(x_{1}, x_{2}\right)=-x_{1}^{2}+x_{2}^{3} .
\end{aligned}
$$

Do cálculo, temos que $\nabla h_{1}\left(x_{1}, x_{2}\right)=(1,0)^{\top}, \nabla g_{2}\left(x_{1}, x_{2}\right)=\left(-2 x_{1}, 1\right)^{\top}$ e $\nabla g_{3}\left(x_{1}, x_{2}\right)=\left(-2 x_{1}, 3 x_{2}^{2}\right)^{\top}$. Desta forma, RCRCQ falha em $x^{*}=(0,0)$. Agora, desde que $C^{\mathcal{W}}\left(x, x^{*}\right)=\left\{(0,0)^{\top}\right\}$, temos que $K_{2}^{\mathcal{W}}(x)=\mathbb{R} \times \mathbb{R}_{+} \times \operatorname{Sym}(2)$. Claramente, $K_{2}^{\mathcal{W}}(x)$ é semicontínua exteriormente.

\subsubsection{Relação com a condição de Baccari-Trad.}

Outra condição de qualificação de segunda ordem conhecida na literatura relacionada com MFCQ, foi é introduzida por Baccari e Trad, (BT05):

Definição 4.3.1. A condição de Baccari-Trad vale em $x^{*} \in \Omega$ se $M F C Q$ vale e o posto dos gradientes das restrições ativas é, no máximo, o número total das restrições ativas menos um.

Apesar da condição de Baccari-Trad garantir a validade de WSONC em um minimizador local, analogamente a CCP2, elas não estão relacionadas.

Exemplo 4.3.3 (A condição de Baccari-Trad não implica CCP2).

Considere em $\mathbb{R}^{2}$ o ponto $x^{*}=(0,0)$ e as restrições de desigualdades definidas pelas funções

$$
\begin{aligned}
& g_{1}\left(x_{1}, x_{2}\right)=-x_{2} ; \\
& g_{2}\left(x_{1}, x_{2}\right)=x_{1}^{2}-x_{2} .
\end{aligned}
$$

Então, a condição de Baccari-Trad vale em $x^{*}$ mas CCP2 falha.

A condição de Baccari-Trad vale em $x^{*}$.

Do cálculo, $\nabla g_{1}\left(x_{1}, x_{2}\right)=(0,-1)^{\top}$ e $\nabla g_{2}\left(x_{1}, x_{2}\right)=\left(2 x_{1},-1\right)^{\top}$. Assim, em $x^{*}=(0,0)^{\top}$, temos que MFCQ vale e que o posto dos gradientes das restrições ativa é exatamente um a menos que o número das restrições ativas (nosso caso, 2).

A condição CCP2 não é satisfeita em $x^{*}$.

Da forma dos gradientes, temos que $C^{\mathcal{W}}\left(x^{*}, x^{*}\right)=\mathbb{R} \times\{0\}$ e $C^{\mathcal{W}}\left(x, x^{*}\right)=\{(0,0)\}$, se $x_{1} \neq x_{1}^{*}$. Assim, para qualquer sequência $\left\{x^{k}\right\}$ com $x^{k} \rightarrow x^{*}$ e $x_{1}^{k}>0$, o cone $K_{2}^{\mathcal{W}}\left(x^{k}\right)=\mathbb{R}_{+} \times \mathbb{R}_{-} \times \operatorname{Sym}(2)$ mas $K_{2}^{\mathcal{W}}\left(x^{*}\right)$ é um subconjunto próprio de $\{0\} \times \mathbb{R}_{-} \times \operatorname{Sym}(2)$. Por conseguinte, CCP2 falha.

Para ver que a condição CCP2 não implica a condição de Baccari-Trad, é suficiente notar que MFCQ+WCR implica CCP2, Proposição 4.3.1, enquanto MFCQ+WCR não implica a condição de Baccari-Trad, (AMS07, contra-exemplo 5.2).

A independência entre CCP2 e a condição Baccari-Trad tem implicações práticas. Devido ao Teorema 4.2.1, a condição de Baccari-Trad não é suficiente para garantir que um ponto limite de uma sequência AKKT2 cumpra WSONC, como mostra o seguinte exemplo.

Exemplo 4.3.4 (AKKT2, sob a condição de Baccari-Trad, não implica WSONC).

Considere o problema de otimização não linear:

Minimizar $f\left(x_{1}, x_{2}\right)=-2 x_{1}^{2}$ sujeito a $g_{1}\left(x_{1}, x_{2}\right)=-x_{2} \leq 0, g_{2}\left(x_{1}, x_{2}\right)=x_{1}^{2}-x_{2} \leq 0$.

Devido ao Exemplo 4.3.3, a condição de Baccari-Trad vale em $x^{*}=(0,0)^{\top}$. Para mostrar que $x^{*}$ é um ponto AKKT2, escolha $x_{1}^{k}:=1 / k, x_{2}^{k}:=x_{1}^{k}, \mu_{1}^{k}:=0, \mu_{2}^{k}:=0, \theta_{2}^{k}:=2\left(x_{1}^{k}\right)^{-2}, \theta_{1}^{k}:=2 \theta_{2}^{k} \mathrm{e}$ $\delta_{k}:=0$. Com essas escolhas temos que $\nabla f\left(x^{k}\right)+\mu_{1}^{k} \nabla g_{1}\left(x^{k}\right)+\mu_{2}^{k} \nabla g_{2}\left(x^{k}\right)=\left(-4 x_{1}^{k}, 0\right)^{\top} \rightarrow(0,0)^{\top} \mathrm{e}$

$$
\nabla^{2} L\left(x^{k}, \mu^{k}\right)+\theta_{1}^{k} \nabla g_{1}\left(x^{k}\right) \nabla g_{1}\left(x^{k}\right)^{T}+\theta_{2}^{k} \nabla g_{2}\left(x^{k}\right) \nabla g_{2}\left(x^{k}\right)^{T}=\left(\begin{array}{cc}
4 & -2 \theta_{2}^{k} x_{2}^{k} \\
-2 \theta_{2}^{k} x_{2}^{k} & 3 \theta_{2}^{k}
\end{array}\right),
$$


onde a última matriz é semi-definida positiva. Observe que WSONC falha em $x^{*}$ e que $x^{*}$ não é uma solução otima para o problema 4.3.15).

Assim, neste exemplo, temos um ponto $x^{*}=(0,0)^{\top}$ que não é uma solução otima e nem satisfaz WSONC, mas ele pode ser atingido por uma sequência AKKT2 (sequência, talvez, gerada pelo método de lagrangiano aumentado ou pelo método SQP regularizado) e como consequência aceito como possível solução. Isto não acontece, se em lugar da condição de Baccari-Trad, consideramos qualquer outra condição de qualificação que implique CPP2, como LICQ, MFCQ+WCR, CRCQ, RCRCQ ou mesmo CCP2.

Resumindo esta seção, definimos uma nova condição de qualificação CPP2 e provamos que CCP2 é uma condição de qualificação para o qual WSONC vale para um minimizador local. A condição CCP2 é estritamente mais fraca que a condição MFCQ+WCR e que a condição RCRCQ. Ainda mais, esta condição é a mínima condição para assegurar que qualquer ponto AKKT2 (ponto limite de uma sequência AKKT2) satisfaz a condição WSONC. Veja o teorema 4.2.1. Isto tem consequência práticas, porque melhora os resultados de convergência para pontos estacionários de segunda ordem de qualquer algoritmo que gere sequências AKKT2, como é o caso do lagrangiano aumentado de (ABMS10) ou o método de regiões de confiança de (DV97). Além disso, esse tipo de resultado não pode ser melhorado usando condições de qualificação mais fracas que CCP2.

\subsection{Nota acerca da condição WSONC}

Nesta seção analisaremos a condição WSONC e forneceremos outra razão do motivo pelo qual a condição WSONC é a condição natural quando analisamos algoritmos práticos com convergência a pontos estacionários de segunda ordem.

Suponha que desejamos uma condição que garanta que todo ponto limite de qualquer sequência AKKT2 satisfaz não somente a condição WSONC mas também a condição forte de segunda ordem, SSONC. Observe que segundo nosso conhecimento, não há algoritmos com convergência a pontos estacionários de segunda ordem em que SSONC vale. Com esse objetivo em mente, definimos a seguinte condição de qualificação no espirito do Teorema 4.2.1, substituindo WSONC por SSONC. Nosso principal objetivo é tentar entender porque não é natural de se esperar que algoritmos práticos gerem sequências cujo pontos limites satisfaçam SSONC.

Definição 4.4.1. Dizemos que a propriedade forte do continuidade do cone de segunda ordem, SCCP2, vale em $x^{*} \in \Omega$ se

$$
\limsup _{x \rightarrow x^{*}} K_{2}^{\mathcal{W}}(x) \subset K_{2}^{\mathcal{S}}\left(x^{*}\right),
$$

onde $K_{2}^{\mathcal{S}}\left(x^{*}\right)$ é o cone:

$$
K_{2}^{\mathcal{S}}\left(x^{*}\right):=\bigcup_{\substack{(\lambda, \mu) \in \mathbb{R}^{m} \times \mathbb{R}_{+}^{p}, \mu_{j}=0 \text { para } j \notin J\left(x^{*}\right)}}\left\{\begin{array}{l}
\left(\sum_{i=1}^{m} \lambda_{i} \nabla h_{i}\left(x^{*}\right)+\sum_{j \in J\left(x^{*}\right)} \mu_{j} \nabla g_{j}\left(x^{*}\right), H\right), \text { tal que } \\
H \preceq \sum_{i=1}^{m} \lambda_{i} \nabla^{2} h_{i}\left(x^{*}\right)+\sum_{j \in J\left(x^{*}\right)} \mu_{j} \nabla^{2} g_{j}\left(x^{*}\right) \text { sobre } C^{S}\left(x^{*}, \mu\right)
\end{array}\right\},
$$

onde $C^{\mathcal{S}}\left(x^{*}, \mu\right)$ é o cone crítico forte 2.0.12).

Usando o cone $K_{2}^{\mathcal{S}}\left(x^{*}\right)$ podemos escrever SSONC de uma forma mais geométrica: SSONC vale em $x^{*}$ se, e somente se o par $\left(-\nabla f\left(x^{*}\right),-\nabla^{2} f\left(x^{*}\right)\right)$ pertence a $K_{2}^{\mathcal{S}}\left(x^{*}\right)$.

Notamos que a condição SCCP2 é mais forte que CCP2, devido a que o cone $K_{2}^{\mathcal{S}}\left(x^{*}\right)$ é um subconjunto de $K_{2}^{\mathcal{W}}\left(x^{*}\right)$. Para ver isto, é suficiente observar que para todo $\mu \geq 0$, o cone crítico fraco $C^{\mathcal{W}}\left(x^{*}\right)$ está incluso em $C^{\mathcal{S}}\left(x^{*}, \mu\right)$.

Seguindo o mesmo raciocínio do Teorema 4.2 .1 temos:

Teorema 4.4.1. Seja $x^{*} \in \Omega$. Então as seguintes condições são equivalentes

(1) SCCP2 vale em $x^{*}$; 
(2) Para qualquer função objetivo $f: \mathbb{R}^{n} \rightarrow \mathbb{R}$ para (2.0.3) tal que AKKT2 é satisfeita em $x^{*}$, então a SSONC vale em $x^{*}$.

Os seguintes exemplos mostram que a condição SCCP2 é tão forte que ela pode falhar mesmo em problemas bem comportados, com estrutura simples e onde a condição LICQ é satisfeita.

Exemplo 4.4.1 (SCCP2 falha para restrições de caixa simples). Considere em $\mathbb{R}^{n}(n \geq 1)$ a restrição de caixa simples $\Omega=\left\{x \in \mathbb{R}^{n}: x \geq 0\right\}$. Então SCCP2 falha em $x^{*}=0$ mas CCP2 vale.

Claramente, a caixa $\Omega$ está definida pelas restrições de desigualdade dadas pelas funções lineares $g_{j}(x)=-x_{j}$, para todo $j \in\{1, \ldots, n\}$. Procederemos com o cálculo do cone $K_{2}^{\mathcal{W}}(x)$. Para $x^{*}=0$, o conjunto das restrições ativas é $J\left(x^{*}\right)=\{1, \ldots, n\}$. Além disso, para todo $x \in \mathbb{R}^{n}$ temos que $\nabla g_{j}(x)=-e_{j}$ e $\nabla^{2} g_{j}(x)=0$, independentemente de $j$. Então temos que

$$
C^{\mathcal{W}}\left(x, x^{*}\right)=\left\{d \in \mathbb{R}^{n}:\left\langle\nabla g_{j}(x), d\right\rangle=0 \text { para todo } j \in\{1, \ldots, n\}\right\}=\{0\},
$$

e como consequência

$$
K_{2}^{\mathcal{W}}(x)=\left\{\left(\sum_{j \in J\left(x^{*}\right)}-\mu_{j} e_{j}, H\right): H \preceq 0 \text { sobre } C^{\mathcal{W}}\left(x, x^{*}\right)=\{0\}, \mu_{j} \geq 0\right\},
$$

se reduz a $K_{2}^{\mathcal{W}}(x)=\mathbb{R}_{-}^{n} \times \operatorname{Sym}(\mathrm{n})$ independentemente de $x$. Assim $K_{2}^{\mathcal{W}}$ é semicontínua exteriormente em $x^{*}$ e CCP2 vale. Ainda mais, $\lim \sup _{x \rightarrow x^{*}} K_{2}^{\mathcal{W}}(x)=\mathbb{R}_{-}^{m} \times \operatorname{Sym}(\mathrm{n})$.

Agora mostraremos que SCPP2 falha em $x^{*}=0$. Para provar que SCCP2 não vale em $x^{*}$, é suficiente encontrar um vetor $\hat{\mu} \in \mathbb{R}_{+}^{n}$ e uma matriz simétrica $H$ tal que $H(w, w)>0$ para algum $w \in C^{\mathcal{S}}\left(x^{*}, \hat{\mu}\right)$. Pois, nesse caso o par $(-\hat{\mu}, H) \in K_{2}^{\mathcal{W}}(x)=\mathbb{R}_{-}^{m} \times \operatorname{Sym}(\mathrm{n})$ e $(-\hat{\mu}, H)$ não pertence a $K_{2}^{\mathcal{S}}\left(x^{*}\right)$. Defina $e:=\sum_{j=1}^{n} e_{j}, \hat{\mu}:=e-e_{1}$ e $H:=e_{1} e_{1}^{T}$. Da definição de cone crítico forte $C^{\mathcal{S}}\left(x^{*}, \hat{\mu}\right)$, temos que $e_{1} \in C^{\mathcal{S}}\left(x^{*}, \hat{\mu}\right)$ e da definição da matriz $H, H\left(e_{1}, e_{1}\right)=\left\|\left\langle e_{1}, e_{1}\right\rangle\right\|^{2}>0$. Desta forma temos que o $\operatorname{par}(-\hat{\mu}, H) \in \lim \sup _{x \rightarrow x^{*}} K_{2}^{\mathcal{W}}(x)$ e não pertence a $K_{2}^{\mathcal{S}}\left(x^{*}\right)$.

Notemos que no exemplo anterior, a condição de independência linear vale em $x^{*}=0$. Já o próximo exemplo mostra que a condição SCPP2 pode valer em problemas onde a independência linear falha.

Exemplo 4.4.2 (SCPP2 não implica LICQ).

Considere em $\mathbb{R}$, o ponto $x^{*}=0$ e o conjunto de restrições de desigualdades definidas por

$$
\begin{aligned}
& g_{1}(x)=-\exp (x)+1 \\
& g_{2}(x)=x
\end{aligned}
$$

Calculando as derivadas e as hessianas, temos que:

$$
\nabla g_{1}(x)=-\exp (x), \quad \nabla^{2} g_{1}(x)=-\exp (x) \quad \text { e } \quad \nabla g_{2}(x)=1, \quad \nabla^{2} g_{2}(x)=0 \text { para todo } x \in \mathbb{R} .
$$

LICQ não vale em $x^{*}$.

Claramente, ambas restrições são ativas em $x^{*}$, além disso da forma dos gradientes, temos que a condição de independência linear não vale em $x^{*}$, de fato não vale em nenhum ponto de $\mathbb{R}$.

$A$ condição SCPP2 vale em $x^{*}$.

Do cálculo, temos que ambos cones $C^{\mathcal{W}}\left(x, x^{*}\right)$ e $C^{\mathcal{S}}\left(x^{*}, \mu\right)$ com $\mu \in \mathbb{R}_{+}^{2}$ são subespaços triviais, isto é, $C^{\mathcal{W}}\left(x, x^{*}\right)=\{0\}$ e $C^{\mathcal{S}}\left(x^{*}, \mu\right)=\{0\}$ para todo $\mu \in \mathbb{R}_{+}^{2}$. Assim, $K^{\mathcal{W}}(x)=\mathbb{R} \times \operatorname{Sym}(1)=K^{\mathcal{S}}\left(x^{*}\right)$ para todo $x \in \mathbb{R}$, o que implica que SCCP2 vale. 
A Figura 4.1 mostra as relações existentes entre as condições de qualificação discutidas nesta subseção.

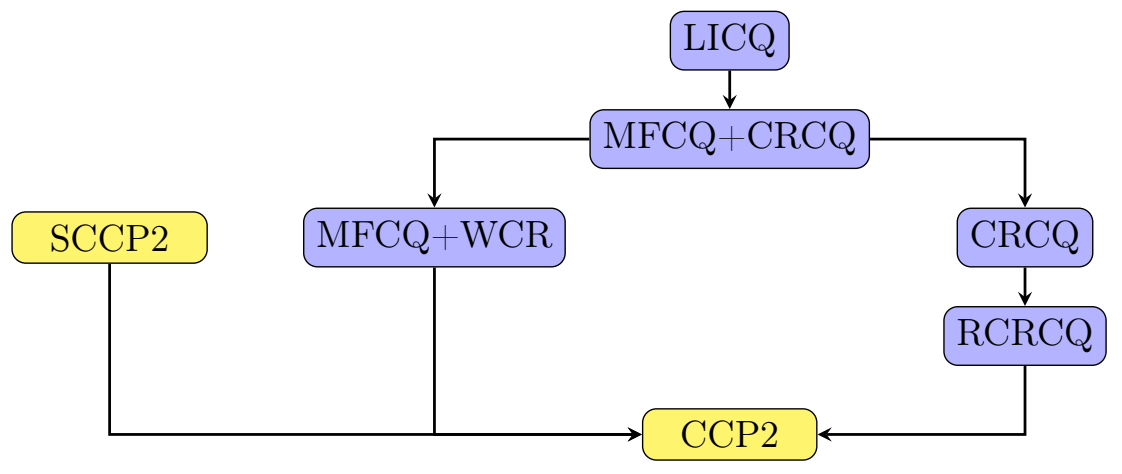

Figura 4.1: Relações das CQs associadas com a convergência a pontos estacionários de segunda ordem. 


\section{Capítulo 5}

\section{Conclusões}

Nesta tese apresentamos uma análise das condições sequenciais de otimalidade e suas relações com as condições de qualificação.

No estudo de muitos métodos numéricos desenvolvidos para resolver os problemas de programação matemática as condições sequenciais de otimalidade têm mostrado ser uma ferramenta útil para a análise de convergência, sob diversas condições de qualificação, por exemplo, LICQ, MFCQ, CPLD, RCPLD, CRCQ, RCRCQ, CRSC, e CPG. Nesta tese, encontramos para cada condição sequencial de otimalidade, a condição de qualificação mínima para qual a condição sequencial implique "KKT ou não CQ" independente da função objetivo. Desta forma, fornecemos a melhor medida de força associada com a condição sequencial de otimalidade e como consequência do respectivo critério de parada. Além disso, estabelecemos as relações das novas condições de qualificação com as outras condições de qualificação encontradas na literatura. Em particular, temos que elas são mais fortes que a condição CPG, mais fracas que a condição de Abadie e independentes da Pseudonormalidade e Quasinormalidade. Acreditamos que essa análise possa servir para entender o comportamento de vários métodos numéricos e também como guia de novas práticas numéricas.

Sob essa paradigma, apresentamos, pela primeira vez, uma condição sequencial de otimalidade de segunda ordem, AKKT2, abrindo a porta para uma unificação da análise de convergência de métodos numéricos com convergência a pontos estacionários de segunda ordem. Como toda condição sequencial de otimalidade, ela satisfaz três propriedades: (i) é uma verdadeira condição de otimalidade, independente de qualquer condição de qualificação, (ii) ela é forte no sentido que implica "WSONC ou não CQ", para condições de qualificação fracas, como por exemplo, MFCQ+WCR ou RCRCQ e (iii) existem algoritmos numéricos que geram sequências cujos pontos limites satisfazem essa condição. Assim, fornecemos um quadro teórico que justifica a ideia de parar os métodos numéricos de segunda ordem quando a condição de segunda ordem é cumprida aproximadamente. Intimamente relacionada com a condição AKKT2, definimos uma nova condição de qualificação, a CCP2. Analisamos as relações da CCP2 com respeito à outras condições de qualificação de segunda ordem conhecidas na literatura. Esse ponto de vista, nós permitiu demonstrar, pela primeira vez segundo nosso conhecimento, que a RCRCQ pode ser usada na análise de convergência, não só de um método, mas para a família de métodos, que geram sequências AKKT2. A condição CPP2 é mais fraca que MFCQ + WCR e, é independente da condição de Baccari-Trad. Este tipo de análise nos permite explicar certos fenómenos que acontecem na prática, por exemplo, podemos concluir e explicar teoricamente que é possível que um método numérico gere uma sequência que converge a um ponto estacionário de segunda ordem, mesmo que os multiplicadores de Lagrange no ponto estacionário sejam ilimitados.

\subsection{Sugestões para Pesquisas Futuras}

Durante o desenvolvimento da tese, várias perguntas e sugestões de pesquisa surgiram, entre as principais que merecem investigação temos as seguintes, que são objeto presente de nossa pesquisa: 
1. Extensão das condições sequenciais fora do âmbito da programação não linear para outros problemas de otimização, como é o caso de programação semi-definida (SDP) ou programação cônica de segunda ordem (SOCP);

2. Procura de novas condições sequenciais de segunda ordem, especialmente adequadas, para métodos de restauração inexata com convergência a pontos estacionários de segunda ordem;

3. Estudo das possíveis aplicações das novas condições de qualificação introduzidas na tese para as várias noções de estabilidade encontradas na literatura. 


\section{Apêndice A}

\section{Uma equivalência de WCR usando ferramentas de análise variacional}

Neste apêndice, mostraremos que a condição WCR pode ser re-escrita usando a linguagem variacional. Para simplificar a prova, consideramos que o conjunto viável é dada só por restrições de desigualdade, isto é, $\Omega:=\left\{x \in \mathbb{R}^{n}: g_{j}(x) \leq 0, j=1, \ldots, m\right\}$.

Antes de provar a Proposição A.0.2. mencionaremos o seguinte lema

Lema A.0.1. A multifunção $C^{\mathcal{W}}\left(\cdot, x^{*}\right)$ é semicontínua interiormente em $x^{*}$ se e somente se, a multifunção definida pelo polar de $C^{\mathcal{W}}\left(\cdot, x^{*}\right)$, é semicontínua exteriormente em $x^{*}$

O Lema A.0.1 é uma aplicação do teorema de separação para conjuntos convexos. O cone polar de $C^{\mathcal{W}}\left(x, x^{*}\right)$ pode ser facilmente calculado, já que $C^{\mathcal{W}}\left(x, x^{*}\right)$ é um subespaço linear. De fato, $C^{\mathcal{W}}\left(x, x^{*}\right)^{\circ}=\left\{\sum_{j \in J\left(x^{*}\right)} \theta_{j} \nabla g_{j}(x): \theta_{j} \in \mathbb{R}\right\}$. Em outras palavras, o cone polar $C^{\mathcal{W}}\left(x, x^{*}\right)^{\circ}$ é o subespaço gerado pelos gradientes $\left\{\nabla g_{j}(x): j \in J\left(x^{*}\right)\right\}$. Continuaremos com o principal resultado desta seção.

Proposição A.0.2. A condição de Posto Constante Fraco (WCR) é equivalente à semi-continuidade interior de $C^{\mathcal{W}}\left(\cdot, x^{*}\right)$ em $x^{*}$

Demonstração. Como consequência do Lema 3.1 de Andreani et al. (AMS07), temos que WCR implica a semi-continuidade interior de $C^{\mathcal{W}}\left(\cdot, x^{*}\right)$ em $x^{*}$.

Para provar a outra implicação usaremos o lema anterior, Lema (A.0.1), assim provaremos que a semi-continuidade exterior de $C^{\mathcal{W}}\left(\cdot, x^{*}\right)^{\circ}$ em $x^{*}$ implica a condição WCR. Para simplificar a notação, denotemos o polar de $C^{\mathcal{W}}\left(\cdot, x^{*}\right)$ como $K_{1}\left(\cdot, x^{*}\right)$.

Suponha, por contradição, que a condição WCR falha em $x^{*}$. Denote por $r$ o posto do conjunto $\left\{\nabla g_{j}\left(x^{*}\right): j \in J\left(x^{*}\right)\right\}$. Seja $\mathcal{J}=\left\{j_{1}, . ., j_{r}\right\} \subset J\left(x^{*}\right)$ tal que $\left\{\nabla g_{j}\left(x^{*}\right): j \in \mathcal{J}\right\}$ é uma base linear de $K_{1}\left(x^{*}, x^{*}\right)$. Por continuidade dos gradientes, temos que $\left\{\nabla g_{j}(x): j \in \mathcal{J}\right\}$ é linearmente independente para todo $x$ em uma vizinhança de $x^{*}$. Como a condição WCR não vale em $x^{*}$, existem uma sequência $\left\{x^{k}\right\}, x^{k} \rightarrow x^{*}$, e um vetor unitário $\omega^{k} \in K_{1}\left(x^{k}, x^{*}\right)$ tal que $\left\{\omega^{k}\right\} \cup\left\{\nabla g_{j}\left(x^{k}\right): j \in \mathcal{J}\right\}$ é um conjunto linearmente independente de $K_{1}\left(x^{k}, x^{*}\right)$, caso contrário o posto não seria constante em uma vizinhança de $x^{*}$. Ainda mais, $\omega^{k}$ pode ser escolhido como ortogonal ao subespaço gerado por $\left\{\nabla g_{j}\left(x^{k}\right): j \in \mathcal{J}\right\}$ para todo $k \in \mathbb{N}$.

Como a sequência $\left\{\omega^{k}\right\}$ é limitada, ela possui uma subsequência convergente. Sem perda de generalidade, podemos assumir que toda a sequência converge, assim $\omega^{k} \rightarrow \omega$ para algum $\omega \in \mathbb{R}^{n}$, ainda mais, como $\omega^{k}$ são vetores unitários, $\|\omega\|=1$. De $\omega^{k} \in K_{1}\left(x^{k}, x^{*}\right)$ e de $\omega^{k} \rightarrow \omega$, concluímos que $\omega \in \lim \sup _{x \rightarrow x^{*}} K_{1}\left(x, x^{*}\right)$. Usando a semi-continuidade exterior de $K_{1}\left(x, x^{*}\right)$ quando $x \rightarrow x^{*}$, temos que $\omega \in K_{1}\left(x^{*}, x^{*}\right)$. Logo, existe $\left\{\theta_{j}^{*}\right\}_{j \in \mathcal{J}}$ tal que

$$
\omega=\sum_{j \in \mathcal{J}} \theta_{j}^{*} \nabla g_{j}\left(x^{*}\right) \quad \text { e } \quad\left\{\theta_{j}^{*}\right\}_{j \in \mathcal{J}} \quad \text { não todos nulos }
$$


Como $\omega^{k}$ é ortogonal a $\left\{\nabla g_{j}\left(x^{k}\right): j \in \mathcal{J}\right\}$, obtemos que

$$
\left\langle\omega, \nabla g_{j}\left(x^{*}\right)\right\rangle=0 \quad \text { para todo } j \in \mathcal{J} .
$$

Agora, substituindo A.0.1 em A.0.2 , podemos re-escrever A.0.2 como o seguinte sistema linear:

$$
G\left(\theta^{*}\right)=0 \quad \text { onde } G=\left(G_{i j}\right)_{i, j \in \mathcal{J}} \text { e } G_{i j}=\left\langle\nabla g_{i}\left(x^{*}\right), \nabla g_{j}\left(x^{*}\right)\right\rangle
$$

onde $\theta^{*}=\left\{\theta_{j}^{*}: j \in \mathcal{J}\right\}$ é uma solução não trivial. Em outras palavras, a matriz grammiana $G$ é singular, mas é bem conhecido que $\left\{\nabla g_{j}\left(x^{*}\right): j \in \mathcal{J}\right\}$ é linearmente independente, se e somente se, a matriz grammiana $G$ associada é não singular. Assim, por A.0.3, $\left\{\nabla g_{j}\left(x^{*}\right): j \in \mathcal{J}\right\}$ não pode ser linearmente independente, o que é uma contradição. 


\section{Referências Bibliográficas}

[ABMS07] R. Andreani, E. G. Birgin, J. M. Martínez e M. L. Schuverdt. Augmented lagrangian methods under the constant positive linear dependence constraint qualification. Mathematical Programming, 111:5-32, 2007.

[ABMS08] R. Andreani, E. G. Birgin, J. M. Martínez e M. L. Schuverdt. On augmented lagrangian methods with general lower-level constraints. SIAM Journal on Optimization, 18:12861309, 2008.

[ABMS10] R. Andreani, E. G. Birgin, J. M. Martínez e M. L. Schuverdt. Second-order negativecurvature methods for box-constrained and general constrained optimization. Computational Optimization and Applications, 45:209-236, 2010.

[AES10] R. Andreani, C. E. Echague e M. L. Schuverdt. Constant-rank condition and second-order constraint qualification. Journal of Optimization theory and Applications, 146(2):255-266, 2010.

[AHM11] R. Andreani, G. Haeser e J. M. Martínez. On sequential optimality conditions for smooth constrained optimization. Optimization, 60(5):627-641, 2011.

[AHRS15] R. Andreani, G. Haeser, A. Ramos e P. J. S. Silva. A second-order sequential optimality condition associated to the convergence of optimization algorithms. Optimization Online, April, 2015.

[AHSS12a] R. Andreani, G. Haeser, M. L. Schuverdt e P. J. S. Silva. A relaxed constant positive linear dependence constraint qualification and applications. Mathematical Programming, $135: 255-273,2012$.

[AHSS12b] R. Andreani, G. Haeser, M. L. Schuverdt e P. J. S. Silva. Two new weak constraint qualifications and applications. SIAM Journal on Optimization, 22:1109-1135, 2012.

[AMRS15] R. Andreani, J. M. Martínez, A. Ramos e P. J. S. Silva. Strict constraint qualifications and sequential optimality conditions for constrained optimization. Optimization Online, November, 2015.

[AMRS16] R. Andreani, J. M. Martínez, A. Ramos e P. J. S. Silva. A cone-continuity constraint qualification and algorithmic consequences. SIAM Journal of Optimization, 26(1):96$110,2016$.

[AMS05] R. Andreani, J. M. Martínez e M. L. Schuverdt. On the relation between constant positive linear dependence condition and quasinormality constraint qualification. Journal of Optimization theory and Applications, 125:473-485, 2005.

[AMS07] R. Andreani, J. M. Martínez e M. L. Schuverdt. On second-order optimality conditions for nonlinear programming. Optimization, 56:529-542, 2007.

[AMS10] R. Andreani, J. M. Martínez e B. F. Svaiter. A new sequential optimality condition for constrained optimization and algorithmic consequences. SIAM Journal of Optimization, 20(6):3533-3554, 2010. 
[AMSS14] R. Andreani, J. M. Martínez, L. T. Santos e B. F. Svaiter. On the behaviour of constrained optimization methods when lagrange multipliers do not exist. Optimization Methods and Software, 29:646-657, 2014.

[Ani00] M. Anitescu. Degenerate nonlinear programming with a quadratic growth condition. SIAM Journal on Optimization, 10(4):1116-1135, 2000.

[Aru91] A. V. Arutyunov. Perturbations of extremum problems with constraints and necessary optimality conditions. Journal of Soviet Mathematics, 54:1342-1400, 1991.

[BBM15] E. G. Birgin, L. F. Bueno e J. M. Martínez. Assessing the reliability of general purpose inexact restoration methods. Journal of Computational and Applied Mathematics, $282: 1-16,2015$.

[Ber82] D. P. Bertsekas. Constrained Optimization and Lagrange Multiplier Methods. Academic Press, 1982.

[Ber99] D. P. Bertsekas. Nonlinear programming. Athenas Scientific, 1999.

[BHM14] L. F. Bueno, G. Haeser e J. M. Martínez. A flexible inexact-restoration method for constrained optimization. Journal of Optimization Theory and Applications, páginas $1-21,2014$.

[Bie10] L. Biegler. NonLinear Programming: Concepts, Algorithms, and Applications to Chemical Processes. MOS-SIAM Series on Optimization, 2010.

[BM14] E. G. Birgin e J. M. Martínez. Practical Augmented Lagrangian Methods for Constrained Optimization. SIAM Publications, 2014.

[BO02] D. P. Bertsekas e A. Ozdaglar. Pseudonormality and a lagrange multiplier theory for constrained optimization. Journal of Optimization Theory and Applications, 114(2):287-343, 2002.

[BO04] D. P. Bertsekas e A. Ozdaglar. The relation between pseudonormality and quasiregularity in constrained optimization. Optimization Methods and Software, 19:493-506, 2004.

[BS98] J. F. Bonnans e A. Shapiro. Optimization problems with perturbations: A guided tour. SIAM Review, 40(2):228-264, 1998.

[BS00] J. F. Bonnans e A. Shapiro. Perturbation Analysis of Optimization Problems. SpringerVerlag, 2000.

[BSS87] R. H. Byrd, R. B. Schnabel e G. A. Shultz. A trust region algorithm for nonlinearly constrained optimization. SIAM Journal of Numerical Analysis, 24:1152-1170, 1987.

[BSS06] M. S. Bazaraa, H. D. Sherali e C. M. Shetty. Practical methods of Optimization: theory and algorithms. John Wiley and Sons, 2006.

[BT05] A. Baccari e A. Trad. On the classical necessary second-order optimality conditions in the presence of equality and inequality constraints. SIAM Journal on Optimization, 15(2):394-408, 2005.

[CG06] L. Chen e D. Goldfarb. Interior point $\ell_{2}$-penalty methods for nonlinear programming with strong global convergence properties. Mathematical Programming, 108:1-26, 2006.

[CGOT98] A. R. Conn, N. I. M. Gould, D. Orban e P. L. Toint. A primal-dual trust-region algorithm for non-convex functions subject to general inequality and linear equality constraints. Nonlinear optimization and related topics, páginas 15-49, 1998. 
[CGT00] A. R. Conn, N. I. M. Gould e Ph. L. Toint. Trust Region Methods. MPS/SIAM Series on Optimization, 2000.

[CLY02] T. F. Coleman, J. Liu e W. Yuan. A new trust-region algorithm for equality constrained optimization. Computacional Optimization and Applications, 21:177-199, 2002.

[Com90] R. Cominetti. Metric regularity, tangent sets and second-order optimality conditions. Applied Mathematics and Optimization, 21:265-287, 1990.

[DEAM97] J. E. Dennis, M. El-Alem e M. C. Maciel. A global convergence theory for general trust-region-based algorithms for equality constrained optimization. SIAM Journal of Optimization, 7(1):177-207, 1997.

[DLP05] G. DiPillo, S. Lucidi e L. Palagi. Convergence to second-order stationary points of a primal-dual algorithm model for nonlinear programming. Mathematics of Operations Research, 30:897-915, 2005.

[DV97] J. E. Dennis e L. N. Vicente. On the convergence theory of trust-region-based algorithms for equality-constrained optimization. SIAM Journal of Optimization, 7(4):927-950, 1997.

[FF10] A. Fischer e A. Friedlander. A new line search inexact restoration approach for nonlinear programming. Computational Optimization and Applications, 46:333-346, 2010.

[Fia83] A. V. Fiacco. Introduction to Sensitivity and Stability Analysis in Nonlinear Programming. Academic Press, INC, volume 165, mathematical in science and engineering edição, 1983.

[FL98] F. Facchinei e S. Lucidi. Convergence to second-order stationary points in inequality constrained optimization. Mathematics of Operations Research, 23:746-766, 1998.

[Fle81] R. Fletcher. Practical methods of Optimization: Constrained Optimization. Wiley, 1981.

[FM68] A. V. Fiacco e G. P. McCormick. Nonlinear Programming Sequential Unconstrained Minimization Techniques. John Wiley, 1968.

[Gau77] J. Gauvin. A necessary and sufficient regularity condition to have bounded multipliers in nonconvex programming. Mathematical Programming, 12:136-138, 1977.

[GKR13] P. E. Gill, V. Kungurtsev e D. P. Robinson. A regularized SQP method with convergence to second-order optimal points. Optimization Online, 2013.

[GR13] P. E. Gill e D. P. Robinson. A globally convergent stabilized SQP method. SIAM Journal of Optimization, 4:1983-2013, 2013.

[GT98] N. I. M. Gould e P. L. Toint. A note on the convergence of barrier algorithms for second-order necessary points. Mathematical programming, 85:433-438, 1998.

[Hae09] G. Haeser. Condiçóes Sequenciais de Otimalidade. Tese de Doutorado, Instituto de Matemática Estatística e Computaçăo Científica, Universidade de Campinas, Brasil, Setembro 2009.

[Hes75] M. R. Hestenes. Optimization Theory: The Finite Dimensional Case. Wiley, 1975.

[HG99] W. W. Hager e M. S. Gowda. Stability in the presence of degeneracy and error estimation. Mathematical Programming, 85:181-192, 1999.

[HS11] G. Haeser e M. L. Schuverdt. On approximate KKT conditions and its extension to continuous variational inequalities. Journal of Optimization Theory and Applications, 149:528-539, 2011. 
[IS12] A. F. Izmailov e M. V. Solodov. Stabilized SQP revisited. Mathematical Programming, páginas 133(93-120), 2012.

[Jan84] R. Janin. Direction derivative of the marginal function in nonlinear programming. Mathematical Programming Studies, 21:127-138, 1984.

[Kun13] V. Kungurtsev. Second-derivative sequential quadratic programming methods for nonlinear optimization. Tese de Doutorado, Department of Mathematics, University of California San Diego, La Jolla, CA, 2013.

[Mar01] J. M. Martínez. Inexact restoration method with lagrangian tangent decrease and new merit function for nonlinear programing. Journal of Optimization Theory and Applications, 11:39-58, 2001.

[MK87] K. G. Murty e S. N. Kabadi. Some NP-complete problems in quadratic and nonlinear programming. Mathematical Programming, 39(2):117-129, 1987.

[Mor06] B. S. Mordukhovich. Variational Analysis and Generalized Differentiation, I: Basis Theory, II: Applications. Springer, 2006.

[MP00] J. M. Martínez e E. A. Pilotta. Inexact restoration algorithm for constrained optimization. Journal of Optimization Theory and Applications, 104:135-163, 2000.

[MP03] J. M. Morguerza e F. J. Prieto. An augmented lagrangian interior-point method using directions of negative curvature. Mathematical Programming, 95 (3):573-616, 2003.

[MS03] J. M. Martínez e B. F. Svaiter. A practical optimality condition without constraint qualifications for nonlinear programming. Journal of Optimization Theory and Applications, 118 No 1:117-133, 2003.

[MS11] L. Minchenko e S. Stakhovski. On relaxed constant rank regularity condition in mathematical programming. Optimization, 60(4):429-440, 2011.

[NW06] J. Nocedal e S. J. Wright. Numerical optimization. Springer, 2006.

[Pen98] J. P. Penot. Second-order conditions for optimization problems with constraints. SIAM Journal of Control and Optimization, 37:303-318, 1998.

[QW00] L. Qi e Z. Wei. On the constant positive linear dependence conditions and its application to SQP methods. SIAM Journal on Optimization, 10:963-981, 2000.

[Rob80] S. M. Robinson. Strongly regular generalized equations. Mathematics of Operations Reseach, 5:43-62, 1980.

[Rus06] A. Ruszczynski. Nonlinear Optimization. Princento University Press, 2006.

[RW09] R. T. Rockafellar e R. Wets. Variational Analysis. Series: Grundlehren der mathematischen Wissenschaften, Vol. 317, 2009.

[Sch06] M. L. Schuverdt. Métodos de Lagrangiano aumentado com convergęncia utilizando a condiçăo de dependęncia linear positiva constante. Tese de Doutorado, Instituto de Matemática Estatística e Computaçăo Científica, Universidade de Campinas, Brasil, Março 2006.

[Spi65] M. Spivak. Calculus on Manifolds: A Modern Approach to Classical Theorems of Advanced Calculus. Addison-Wesley Publishing Company, 1965. 